Luiz Fernando de Souza Dias

\title{
ESTUDO DA LOCALIZAÇÃO OTIMIZADA DE EQUIPAMENTOS PARA DETECÇÃO DE CONTAMINAÇÃO EM REDES DE DISTRIBUIÇÃO DE ÁGUA
}

\begin{abstract}
Dissertação apresentada à Escola de Engenharia de São Carlos da Universidade de São Paulo, como parte dos requisitos para obtenção do título de Mestre em Hidráulica e Saneamento.
\end{abstract}

Orientadora: Prof ${ }^{a}$ Dra. Luisa Fernanda Ribeiro Reis

São Carlos

2006 
Ficha catalográfica preparada pela Seção de Tratamento da Informação do Serviço de Biblioteca - EESC/USP

Dias, Luiz Fernando de Souza

D541e partudo da localização otimizada de equipamentos
para detecção de contaminação em redes de distribuição de água / Luiz Fernando de Souza Dias. -- São Carlos, 2006 .

Dissertação (Mestrado) -- Escola de Engenharia de São Carlos-Universidade de São Paulo, 2006.

Área: Hidráulica e Saneamento.

Orientador: Profa. Dra. Luisa Fernanda Ribeiro Reis.

1. Qualidade da água - monitoramento. 2. Qualidade da água - detecção de contaminantes. 3. Redes de distribuição de água. 4. Algoritmos genéticos multiobjetivo. I. Título. 


\section{FOLHA DE JULGAMENTO}

Candidato: Engenheiro LUIZ FERNANDO DE SOUZA DIAS

Dissertação defendida e julgada em 06-04-2006 perante a Comissão Julgadora:

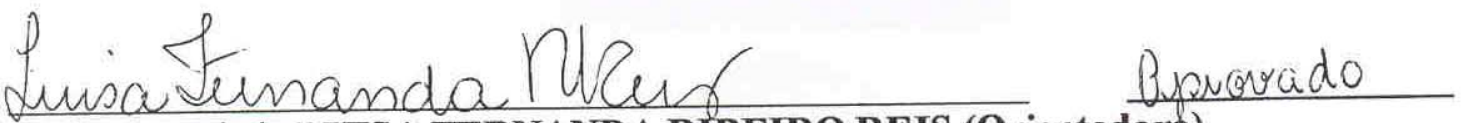
Profa. Associada LUISA FERNANDA RIBBEIRO REIS (Orientadora)

(Escola de Engenharia de São Carlos/USP)

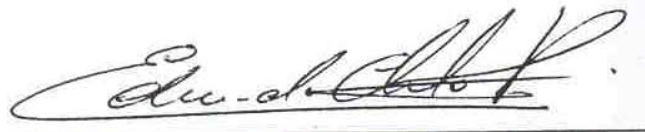

Afruvado

Prof. Titular EDUARDO CLETO PIRES

(Escola de Engenharia de São Carlos/USP)

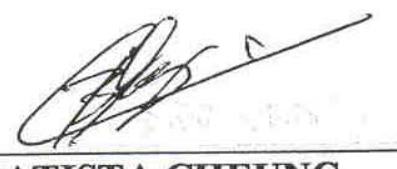

APRONADO

Dr. PETER batista CHEUNG

(Fundação Universidade Federal de Mato Grosso do Sul/ FUFMS)

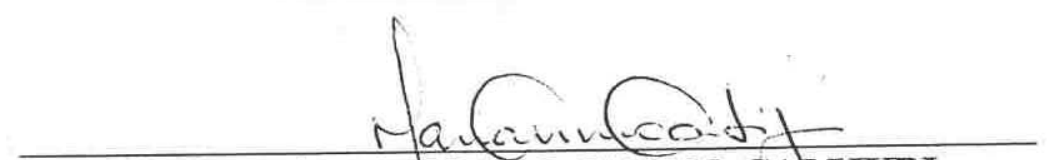

Profa. Titular MARIA DO CARMO CALIJURI

Coordenadora do Programa de Pós-Graduação em

Engenharia (Hidráulica e Saneamento) e

Presidente da Comissão de Pós-Graduação 


\section{AGRADECIMENTOS}

À minha orientadora, Professora Luisa Fernanda Ribeiro Reis, pela sua capacidade de auxiliar, ajudar e apoiar, além de sua dedicação a este trabalho.

Aos meus pais, José Carlos e Elizabeth, pelo seus esforços, amor e educação.

Ao meu filho, Luiz Gustavo, pelo amor, carinho, lazer e descontração.

À minha companheira e amiga, Vânia, pelo carinho, compreensão e ajuda nos momentos que mais precisei.

Ao Dr. Peter Batista Cheung, pela ajuda na implementação do código computacional e dicas apresentadas.

Aos colegas do Laboratório de Simulação Numérica, pela colaboração e companheirismo.

Aos professores, colegas e funcionários do Departamento de Hidráulica e Saneamento da EESC-USP, pelo aprendizado, conhecimento e colaboração.

À Coordenadoria de Aperfeiçoamento de Pessoal de Nível Superior CAPES, pela bolsa de estudo concedida.

E a todas as outras pessoas que de alguma maneira contribuíram para a realização deste trabalho. 


\section{RESUMO}

DIAS, L. F. S. (2006). Estudo da Localização Otimizada de Equipamentos para Detecção de Contaminação em Rede de Distribuição de Água. São Carlos, 2006. 144p. Dissertação (Mestrado) - Escola de Engenharia de São Carlos, Universidade de São Paulo, São Carlos, 2006.

A qualidade da água de abastecimento é de vital importância à saúde da população dos núcleos urbanos do mundo todo. Por essa razão, muitas pesquisas enfocam esse tema. Além disso, os ataques terroristas recentes ocorridos nos Estados Unidos e Europa, vêm fomentando a antiga preocupação relativa a possíveis injeções de contaminantes em redes de distribuição de água para abastecimento, evidenciando a importância da efetiva vigilância de tais sistemas.

O presente trabalho investiga a rede de monitoramento ótima para detecção de injeções intencionais de poluentes e/ou contaminantes em concentrações e/ou quantidades suficientes para que se propaguem nas direções do fluxo da água no interior das redes, do ponto de vista de objetivos múltiplos.

A metodologia aqui apresentada representa uma extensão de propostas anteriores e é demonstrada ilustrativamente, através de redes já utilizadas na literatura. Com base no conceito da rede auxiliar proposto por Kessler et al. (1998), propõe-se o emprego de Algoritmos Genéticos Multiobjetivo para considerar os níveis de serviço em termos do volume consumido, do tempo e da extensão da rede atingida antes da detecção. São criadas matrizes de poluição para os níveis de serviço considerados e, então, o algoritmo genético multiobjetivo SPEA é aplicado para identificar as soluções não-dominadas, em conformidade com o conceito de otimalidade de Pareto. Os resultados demonstram o potencial do método proposto em identificar tais soluções.

Palavras-chave: monitoramento, qualidade da água, detecção de contaminantes, redes de distribuição de água, algoritmos genéticos multiobjetivo. 


\begin{abstract}
DIAS, L. F. S. (2006). Study of Optimized Localization of Equipments for Contamination Detection in Water Distribution Networks. São Carlos, 2006. 144p. Dissertação (Mestrado) -São Carlos School of Engineering, University of São Paulo, São Carlos, 2006.

The water supply quality is very important to the healthy of urban nucleus populations around the world. This is the reason why many researches focus on such theme. Besides this, recent terrorist attacks occurred in USA and Europe, have incited the old apprehension related to possible deliberate intrusions of contaminants into the water supply networks, making evident the importance of the effective vigilance of such systems.
\end{abstract}

This work investigates the optimal monitoring network for detection of deliberate intrusions of pollutes and/or contaminants at concentrations e/or quantities enough for propagation inside the networks, on the point of view of multiple objectives.

The method here proposed represents an extension to earlier proposals and is demonstrated with the support of networks from literature. Based on the concept of auxiliary network proposed by Kessler et al. (1998), a multiobjective genetic algorithm is used in order to consider the levels of service in terms of the consumed volume, time period and length of the network reached before detection. Pollution matrixes are built for the levels of service considered and the multiobjective genetic algorithm SPEA applied in the identification of the non-dominated solutions, according to the Paretto optimality concept. The results demonstrate the potential of the method in the identification of such solutions.

Keywords: monitoring, water quality, detection of contaminants, water distribution network, multiobjective genetic algorithms. 
Estudo da Localização Otimizada de Equipamentos em Rede

de Distribuição de Água para Detecção de Contaminação

\section{LISTA DE FIGURAS}

Figura 5.1 - Rede Net1 32

Figura 5.2 - Grafo exemplo___ 37

Figura 5.3 - Rede auxiliar para Tempo de viagem da Rede Net1 __ 43

Figura 5.4 - Rede auxiliar para Extensão de viagem da Rede Net1_ 43

Figura 5.5 - Solução 1 da Rede Net1 para 1 equipamento considerando detecções repetidas. ___ 61

Figura 5.6 - Solução 1 da Rede Net1 para 2 equipamento considerando detecções repetidas. ___ 61

Figura 5.7 - Solução 2 da Rede Net1 para 2 equipamento considerando detecções repetidas. ___ 62

Figura 5.8 - Solução 1 da Rede Net1 para 3 equipamento considerando detecções repetidas. __ 62

Figura 6.1 - Rede esquemática da Anytown __ 66

Figura 6.4 - Solução 1 da Rede Anytown para 1 equipamento considerando detecções repetidas. _ 80

Figura 6.5 - Solução 1 da Rede Anytown para 2 equipamento considerando detecções repetidas. _ 80

Figura 6.6 - Solução 2 da Rede Anytown para 2 equipamento considerando detecções repetidas. __ 81

Figura 6.7 - Solução 3 da Rede Anytown para 2 equipamento considerando detecções repetidas. __ 81

Figura 6.8 - Solução 1 da Rede Anytown para 3 equipamento considerando detecções repetidas. __ 82

Figura 6.9 - Solução 2 da Rede Anytown para 3 equipamento considerando detecções repetidas. _ 82

Figura 6.10 - Solução 3 da Rede Anytown para 3 equipamento considerando detecções repetidas. __ 83

Figura 6.11 - Solução 1 da Rede Anytown para 4 equipamento considerando detecções repetidas. __ 83

Figura 6.12 - Solução 2 da Rede Anytown para 4 equipamento considerando detecções repetidas. _ 84

Figura 6.13 - Solução 3 da Rede Anytown para 4 equipamento considerando detecções repetidas. _ 84

Figura 6.14 - Solução 4 da Rede Anytown para 4 equipamento considerando detecções repetidas. __ 85

Figura 7.1 - Gráfico Valores máximos de F1, F2 e F3 x Número de Equipamentos para Rede Net1 não considerando detecções repetidas.

Figura 7.2 - Gráfico Valores máximos de F1, F2 e F3 x Número de Equipamentos para Rede Net1 considerando detecções repetidas.

Figura 7.3 - Gráfico Valores máximos de F1, F2 e F3 x Número de Equipamentos para Rede Anytown não considerando detecções repetidas $\left(V C=468,10 \mathrm{~m}^{3}\right)$.

Figura 7.4 - Gráfico Valores máximos de F1, F2 e F3 x Número de Equipamentos para Rede Anytown considerando detecções repetidas $\left(\mathrm{VC}=468,10 \mathrm{~m}^{3}\right)$.

Figura 7.5 - Gráfico Valores máximos de F1, F2 e F3 x Número de Equipamentos para Rede Anytown não considerando deteç̧ões repetidas $\left(V C=1245,15 \mathrm{~m}^{3}\right)$.

Figura 7.6 - Gráfico Valores máximos de F1, F2 e F3 x Número de Equipamentos para Rede Anytown considerando detecções repetidas $\left(V C=1245,15 \mathrm{~m}^{3}\right)$. 
Estudo da Localização Otimizada de Equipamentos em Rede

de Distribuição de Água para Detecção de Contaminação

\section{LISTA DE TABELAS}

Tabela 1.1 - Principais doenças associadas com a água. 1

Tabela 1.2 - Efeitos de algumas substâncias químicas nos seres humanos. __ 3

Tabela 2.1 - Padrão de Potabilidade para algumas substâncias químicas que apresentam risco à saúde.

Tabela 2.2 - Número mínimo de amostragens, em mananciais superficiais, para fins de análises físicas, químicas e de radioatividade, em função do plano de amostragem e da população abastecida. ___ 11 Tabela 2.3 - Freqüência mínima de amostragem para o controle da qualidade da água de sistema de abastecimento, para fins de análises físicas, químicas e de radioatividade, em função do plano de amostragem e da população abastecida em mananciais superficiais.

Tabela 5.1 - Característica do Tanque da rede Net1.

Tabela 5.2 - Características de demanda padrão para 24 horas da rede Net1.

Tabela 5.3 - Maximização com 3 objetivos

Tabela 5.4 - Resultados da Rede Net1 não considerando detecções repetidas. _— 60

Tabela 5.5 - Resultados da Rede Net1 considerando detecções repetidas __ 60

Tabela 6.1 - Características dos tubos da Rede Anytown ___ 67

Tabela 6.2 - Características dos nós da Rede Anytown _ـ 68

Tabela 6.3 - Características dos Tanques da Rede Anytown _ 68

Tabela 6.4 - Características da bomba da Rede Anytown__ 68

Tabela 6.5 - Padrão diário de demanda para a Rede Anytown _ـ 68

Tabela 6.6 - Resultados da Rede Anytown não considerando detecções repetidas: Nível VC $=468,10 \mathrm{~m}^{3}$.

Tabela 6.7 - Resultados da Rede Anytown considerando detecções repetidas: Nível VC = 468,10 m². 77 Tabela 6.8 - Resultados da Rede Anytown não considerando detecções repetidas: Nível VC = 1245,15 $m^{3}$.

Tabela 6.9-Resultados da Rede Anytown considerando deteç̧ões repetidas: Nível VC = 1245,15 $\mathrm{m}^{3} .79$

Tabela 7.1 - Resultados da Rede Net1 não considerando detecções repetidas. __ 87

Tabela 7.2 - Resultados da Rede Net1 considerando detecções repetidas. ___ 88

Tabela 7.3 - Resultados da Rede Anytown não considerando detecções repetidas: Nível VC $=468,10 \mathrm{~m}^{3}$.

Tabela 7.4 - Resultados da Rede Anytown considerando detecções repetidas: Nível VC = 468,10 $\mathrm{m}^{3}$. 91 Tabela 7.5 - Resultados da Rede Anytown não considerando detecções repetidas: Nível VC = 1245,15 $m^{3}$.

Tabela 7.6 - Resultados da Rede Anytown considerando detecções repetidas: Nível VC =1245,15 $\mathrm{m}^{3} .93$ 


\section{LISTA DE ABREVIATURAS E SIGLAS}

ANVISA

AG

EESC

ID

LOS

MOGA

MOMGA

NSGA

NSGA-II

PAES

PL

PLEDECORDA

RDA

RPM

SCADA

SPEA

U.S. EPA

USP
Agência Nacional de Vigilância Sanitária

Algoritmo Genético

Escola de Engenharia de São Carlos

Identificação

Level Of Service

MultiObjective Genetic Algorithm

MultiObjective Messy Genetic Algorithm

Non-dominated Sorting Genetic Algorithm

elitist Non-dominated Sorting Genetic Algorithm

Pareto-Archied Evolution Strategy

Programação Linear

Programa de Localização de Equipamentos para DEtecção de COntaminação em Rede de Distribuição de Água

Rede de Distribuição de Água

Randomized Pollution Matrix

Supervisory Control and Data Acquisition

Strength Pareto Evolutionary Algorithm

United States Environmental Protection Agency

Universidade de São Paulo 


\section{SUMÁRIO}

RESUMO

ABSTRACT

LISTA DE FIGURAS

LISTA DE TABELAS iii

LISTA DE ABREVIATURAS E SIGLAS iv

1. INTRODUÇÃO

1.1. Justificativa

1.2. Objetivo

2. REVISÃO DA LITERATURA__ 9

3. MATERIAIS_ 23

3.1. Microcomputador__ 23

3.2. Softwares e ferramentas___ 23

3.2.1. Builder C++ (Borland)___ 23

3.2.2. MOMHLIB++ (Jaszkiewicz, 1999) - Algoritmos Genéticos Multiobjetivo 23

3.2.3. Simulador EPANET__ 24

4. O MÉTODO _ 28

5. METODOLOGIA APLICADA: Rede Net1_ 30

5.1. Simulação Hidráulica _ 30

5.1.1. Caracterização das Redes _ 31

5.1.2. Modelagem da Rede__ 33

5.2. Inicialização do PLEDECORDA_EPA___ 33

5.2.1. Parâmetros de Entrada - Níveis de Serviço _ 34

5.3. Construção da Rede Auxiliar___ 34

5.4. Determinação dos Caminhos Mais Curtos __ 35

5.4.1. Algoritmo de Floyd (1962)___ 35

5.5. Determinação das Matrizes de Poluição___ 44

5.5.1. Identificação dos objetivos___ 44

5.5.1.1. Construção da Matriz Poluição para Volume Consumido__ 46

5.5.1.2. C Construção da Matriz Poluição para Tempo de Deteç̧ão _ _ 47

5.5.1.3. Construção da Matriz Poluição para Extensão da Contaminação _ _ 48

5.6. Inicialização do Programa PLEDECORDA_MOM__ 49 
5.7. Determinação dos Nós de Monitoramento __ 50

5.7.1. O Problema a ser tratado__ 50

5.7.1.1. O princípio dos Algoritmos Genéticos (AGs) _ 51

5.7.1.2. Os AGs Multiobjetivo___ 52

5.7.1.3. SPEA__ 54

5.7.2. Implementação dos AGs multiobjetivo sobre as Matrizes de Poluição _ 57

5.7.2.1. Funções de Aptidão __ 57

5.7.2.2. Representação das soluções___ 59

6. ESTUDO DE CASO: Rede Anytown 66

6.1. Simulação Hidráulica _ 66

6.2. Inicialização do PLEDECORDA_EPA___ 69

6.2.1. Parâmetros de Entrada - Níveis de Serviço __ 69

6.3. Construção das Redes Auxiliares e dos Caminhos Mais Curtos _ 69

6.4. Determinação das Matrizes de Poluição_ 72

6.5. Inicialização do Programa PLEDECORDA_MOM___ 75

6.6. Determinação dos Nós de Monitoramento _ 75

7. DISCUSSÃO DOS RESULTADOS _ 86

7.1. Rede Net1 86

7.2. Rede Anytown_ 89

8. CONCLUSÕES 95

REFERENCIAS _ 98

Anexo I-REDE.INP (Rede Net1): Dados de Entrada __ 103

Anexo II - PLEDECORDA_EPA.TXT (Rede Net1): Saídas das Matrizes de Poluição 107

Anexo III - PLEDECORDA_MOM.TXT (Rede Net1): Saídas dos Nós escolhidos 113 Anexo IV - REDE.INP (Rede Anytown): Dados de Entrada _ 115

Anexo V-PLEDECORDA_EPA.TXT (Rede Anytown): Saídas das Matrizes de Poluição: Nível de Serviço 1 - Volume Consumido: 468,10m

Anexo VI - PLEDECORDA_MOM.TXT (Rede Net1): Saídas dos Nós escolhidos: Nível de Serviço 1 - Volume Consumido: 468,10m³

Anexo VII - PLEDECORDA_EPA.TXT (Rede Anytown): Saídas das Matrizes de Poluição: Nível de Serviço 1 - Volume Consumido: 1245,15m³

Anexo VIII - PLEDECORDA_MOM.TXT (Rede Anytown): Saídas dos Nós escolhidos: Nível de Serviço 1 - Volume Consumido: 1245,15m³ 


\section{INTRODUÇÃO}

$\mathrm{Na}$ condição de solvente universal, a água pode arrastar consigo uma variedade de substâncias e microrganismos patogênicos e assim, representar sérios riscos à saúde da população abastecida por ela.

Segundo GALAL-GORCHEV (1996), nos países em desenvolvimento, onde se enquadra o Brasil, estima-se que $80 \%$ das doenças e mais de um terço das mortes estão associadas à utilização e consumo de águas contaminadas. Neste contexto, VON SPERLING (1996) apresenta os principais microorganismos causadores de doenças relacionadas com a água. As doenças, bem como os seus agentes causais e os respectivos sintomas estão relacionados na Tabela 1.1.

Tabela 1.1 - Principais doenças associadas com a água.

\begin{tabular}{|c|c|c|}
\hline Doença & $\begin{array}{c}\text { Agente Causal } \\
\end{array}$ & Sintomas \\
\hline \multicolumn{3}{|c|}{ Ingestão de água contaminada } \\
\hline Disenteria bacilar & Bactéria Shigella dysenteriae & Forte diarréia. \\
\hline Cólera & Bactéria Vibrio cholerae & $\begin{array}{l}\text { Forte diarréia, desidratação, alta } \\
\text { taxa de mortalidade. }\end{array}$ \\
\hline Leptospirose & Bactéria Leptospira & Icterícia, febre. \\
\hline Salmonelose & Bactéria Salmonella & Febre, náusea, diarréia. \\
\hline Febre Tifóide & Bactéria Salmonella typhi & $\begin{array}{l}\text { Febre alta, diarréia, ulceração do } \\
\text { intestino delgado. }\end{array}$ \\
\hline Disenteria amebiana & Protozoário Entamoeba histolytica & $\begin{array}{lr}\text { Diarréia prolongada } & \text { com } \\
\text { sangramento, abscessos } & \text { no } \\
\text { fígado e intestino fino. } & \\
\end{array}$ \\
\hline Giardíase & Protozoário Giardia lamblia & $\begin{array}{l}\text { Diarréia, náusea, indigestão, } \\
\text { flatulência. }\end{array}$ \\
\hline Hepatite infecciosa & Vírus da hepatite tipo A & Icterícia, febre. \\
\hline Gastroenterite & $\begin{array}{l}\text { Vírus: enterovírus, parvovírus, } \\
\text { rotavírus. }\end{array}$ & Diarréia leve a forte. \\
\hline Paralisia infantil & Vírus Poliomielites virus & Paralisia. \\
\hline \multicolumn{3}{|c|}{ Contato com água contaminada } \\
\hline Escabiose & Sarna Sarcoptes scbiei & Úlceras na pele \\
\hline Tracoma & Clamídea Chlamydia tracomatis & $\begin{array}{l}\text { Inflamação nos olhos, cegueira } \\
\text { parcial ou total. }\end{array}$ \\
\hline
\end{tabular}

Fonte: VON SPERLING, 1996. 
Há, porém, uma grande dificuldade na detecção de bactérias, protozoários e vírus em uma amostra de água. Isso se dá pelo fato de estarem presentes em pequenas concentrações. Para solucionar este problema, realiza-se a contagem de organismos denominados organismos indicadores de contaminação fecal, que mesmo não sendo patogênicos, fornecem indicações de possível contaminação fecal e do conseqüentemente potencial da água em transmitir doenças. Os principais representantes destes organismos são as bactérias do grupo coliforme. A Portaria 518/04 do Ministério da Saúde define que a presença do coliforme termotolerante Eschericlia coli, de origem exclusivamente fecal, é o mais específico indicador de contaminação fecal recente e de eventual presença de organismos patogênicos.

Outras doenças que podem afetar a população através da veiculação hídrica são as causadas pela presença de substâncias tóxicas ou nocivas e que muitas vezes não são percebidas pelo gosto, aparência ou cheiro, mas podem provocar males e até mesmo epidemias.

Verifica-se, portanto, a necessidade de se conhecer as substâncias presentes na água e as que podem vir a contaminá-la, além de suas concentrações limites e suas reações em organismos vivos. Também é de extrema importância que as autoridades competentes façam o monitoramento destes parâmetros, seguindo um plano pré-definido, evitando assim que casos de epidemias e acidentes ambientais possam colocar em risco a saúde da população. 
DI BERNARDO, 1983).

Tabela 1.2 - Efeitos de algumas substâncias químicas nos seres humanos.

\begin{tabular}{|c|c|}
\hline Substância Química & Problemas \\
\hline Ferro e Manganês & $\begin{array}{l}\text { Em pequenas concentrações causam cor na água e em elevadas } \\
\text { concentrações causam sabor e odor. }\end{array}$ \\
\hline Cloretos & Em determinadas concentrações imprimem sabor salgado a água. \\
\hline Nitrogênio & $\begin{array}{l}\text { Na forma de nitrato está associado a doenças como a metahemoglobina } \\
\text { (síndrome do bebê azul). Na forma de amônia é tóxica aos peixes. }\end{array}$ \\
\hline \multicolumn{2}{|l|}{ Metais Pesados } \\
\hline Alumínio & $\begin{array}{l}\text { Constipação intestinal, perda de energia, cólicas abdominais, hiperatividade } \\
\text { infantil, perda de memória, dificuldade de aprendizado, osteoporose, } \\
\text { raquitismo e convulsões. Doenças relacionadas: Alzheimer e Parkinson. }\end{array}$ \\
\hline Arsênico & $\begin{array}{l}\text { Transtornos gastrointestinais, espasmos músculo-viscerais, náuseas, } \\
\text { diarréias, inflamações da boca e garganta, dores abdominais. }\end{array}$ \\
\hline Cádmio & $\begin{array}{l}\text { Metal cancerígeno, provoca elevação da pressão sanguínea e aumento do } \\
\text { coração. Queda da imunidade. Aumento da próstata. Enfraquecimento } \\
\text { ósseo. Dores nas articulações. Anemia. Enfizema pulmonar. Osteoporose. } \\
\text { Perda de olfato. Perda do desempenho sexual. }\end{array}$ \\
\hline Chumbo & $\begin{array}{l}\text { Irritabilidade e agressividade, indisposição, dores de cabeça, convulsões, } \\
\text { fadiga, sangramento gengival, dores abdominais, náuseas, fraqueza } \\
\text { muscular, abnubilação mental, perda de memórias, insônia, pesadelos, } \\
\text { acidente vascular cerebral inespecífico, alterações de inteligência, } \\
\text { osteoporose, doenças renais, anemias, problemas de coagulação. }\end{array}$ \\
\hline Mercúrio & $\begin{array}{l}\text { Depressão, fadiga, tremores, síndrome do pânico, parestesias, descontrole } \\
\text { motor, andar lateral, dificuldade de fala, perda de memória, perda do } \\
\text { desempenho sexual, estomatite, dentes soltos, dor e paralisia de } \\
\text { extremidades, dor de cabeça, anorexia em crianças, alucinações, vômitos, } \\
\text { febre, dificuldades de mastigação, sudorese e perda do senso da dor. }\end{array}$ \\
\hline Níquel & $\begin{array}{l}\text { Metal cancerígeno, pode causar: dermatite de contato, gengivites, erupções } \\
\text { na pele, estomatite, tonturas, dores articulares, osteoporose e fadiga } \\
\text { crônica. }\end{array}$ \\
\hline Bário & Hipertensão arterial, doenças cardiovasculares, fadiga e desânimo. \\
\hline \multicolumn{2}{|c|}{ Compostos Orgânicos Voláteis } \\
\hline Benzeno & Causa problemas cancerígenos e alterações do número de cromossomos. \\
\hline Monoclorobenzeno & Solvente usado na produção de pesticida. Na água afeta fígado e rins. \\
\hline Diclorobenzeno & $\begin{array}{l}\text { Solvente usado na produção de pesticida e corante que afeta pulmão, } \\
\text { fígado, rins e causa envenenamento do sangue. }\end{array}$ \\
\hline Tetracloreto de carbono & $\begin{array}{l}\text { Usado na fabricação de clorofluormetano (extintores, solventes, etc) é um } \\
\text { provável causador de câncer. }\end{array}$ \\
\hline 1,2 dicloroetano & $\begin{array}{l}\text { Usado para fabricar detergentes e inseticidas. Causam problemas hepáticos } \\
\text { e renais. }\end{array}$ \\
\hline \multicolumn{2}{|l|}{ Agrotóxicos } \\
\hline Organoclorados & Acumulam-se do Sistema Nervoso Central causando danos. \\
\hline $\begin{array}{l}\text { Organofosforados } \\
\text { carbamatos }\end{array}$ & Alteram o funcionamento de glândulas, músculos e cérebro. \\
\hline Piretróides & $\begin{array}{l}\text { Causam reações no Sistema Nervoso Central causando convulsões e } \\
\text { paralisia. }\end{array}$ \\
\hline
\end{tabular}

Fonte: VON SPERLING, 1996; DI BERNARDO, 1983. 
Conhecidos os efeitos benéficos da distribuição da água tratada e os problemas decorrentes da distribuição de água contaminada, a água distribuída deve ser desinfectada e apresentar um residual de desinfetante nos pontos de consumo, com o objetivo de destruir pequenas concentrações de microrganismos que venham a penetrar no sistema. Se a contaminação for elevada, o residual de desinfetante servirá indiretamente como indicador, pois sua ausência será facilmente identificada (DI BERNARDO, 1983). Assim, outro produto químico, de grande importância sanitária é o cloro, geralmente usado no processo da desinfecção da água. Existe, no entanto, uma crescente preocupação com os seus subprodutos, uma vez que reações do cloro com a matéria orgânica presente na água podem dar origem a produtos indesejáveis tais como os trihalometanos que são compostos cancerígenos.

Casos de contaminação não são inéditos no Brasil. Em 2000 (CALDERARO et al., 2001), dezesseis pacientes submetidos à hemodiálise em um hospital de Minas Gerais apresentaram reações hemolíticas compatíveis com sintomas de intoxicação por cloro e cloramina. Naquele período, fora efetuada a instalação de mais um reservatório no sistema existente, que passou a abastecer o anel de distribuição de água para as máquinas de hemodiálise. Durante a reforma, as instalações foram abastecidas por um reservatório antigo, cujo serviço de desinfecção era feito à base de cloro, por firma terceirizada. O procedimento não foi acompanhado pelos funcionários do centro de hemodiálise, que não se fizeram presentes durante o enxágüe do circuito e os testes de residual de cloro. Nas sessões seguintes, alguns pacientes 
apresentaram sintomas compatíveis com intoxicação por cloro. Os sintomas foram dores torácicas, lombares e abdominais, diarréia, hemólise, coagulação no capilar, calor e rubor, alguns destes ocorrendo simultaneamente em alguns pacientes. Realizadas as análises, detectou-se uma concentração excepcionalmente elevada $(>2,75 \mathrm{mg} / \mathrm{l})$ de cloro total na água utilizada no processamento, que se constatou ser originária do reservatório antigo, que, depois da reforma, passou a abastecer o local. Uma pesquisa de FLUCK et al. (1999) indica que concentrações elevadas de cloro e cloraminas em águas de hemodiálise podem provocar graves reações em pacientes, com possíveis danos à saúde. Essa constatação realça a importância da efetiva vigilância dos sistemas nem sempre observada como o único instrumento com eficácia para evitar agravos à saúde dos pacientes.

Outro estudo sobre contaminação (CAVALCANTI, 2003), reporta que amostras de ostras comercializadas em Recife, Brasil, foram monitoradas em relação ao conteúdo de elementos traço $(\mathrm{Hg}, \mathrm{Zn}, \mathrm{Fe}, \mathrm{Cu}$ e $\mathrm{Mn})$ durante o período de um ano (março/2001 a fevereiro/2002). O mercúrio, um dos elementos que apresentam maior risco à saúde pública, foi o principal contaminante encontrado nas ostras. A avaliação dos níveis de mercúrio em frutos do mar é um importante fator para avaliar o risco de contaminação por mercúrio em pessoas não ocupacionalmente expostas. Chegou-se à conclusão de que há muitos anos não é feito um monitoramento dos níveis de mercúrio do canal de Santa Cruz, onde as ostras foram coletadas, pelos órgãos ambientais de Pernambuco. 
Muitos trabalhos ignoram aspectos importantes relacionados à qualidade da água no que se refere à presença de substâncias e microorganismos indesejáveis ou em concentrações inadequadas. A má qualidade da água nas redes de distribuição é decorrente principalmente (KESSLER et al., 1998): (a) da redução (exemplo: cloro residual) ou aumento (exemplo: trialometanos totais e bactérias) de substâncias não-conservativas durante a sua veiculação no interior desses sistemas e (b) da intrusão acidental ou intencional de poluentes ou contaminantes. Destaca-se aqui a preocupação com a notória vulnerabilidade das redes às ameaças físicas, químicas e biológicas, que vieram à tona a partir de incidentes terroristas nos EUA e Europa, e que vêm forçando ações no sentido de promover a segurança de infra-estruturas de abastecimento.

Visando a proteção da população abastecida, as redes de abastecimento devem ser dotadas de equipamentos de monitoramento contínuo de qualidade da água. Idealmente, todo nó do sistema deveria ser monitorado para promover a máxima proteção da saúde pública. Porém, por razões de economia, admite-se que o monitoramento possa ser realizado com um número reduzido de equipamentos, localizados em pontos estratégicos da rede, de forma a poder auxiliar na detecção de contaminantes, na identificação das suas fontes e na conseqüente redução do risco à população. No caso específico da intrusão externa de poluentes, os equipamentos de monitoramento devem ser projetados para emitir um sinal de alarme quando os poluentes forem detectados. 


\subsection{Justificativa}

A determinação do número de equipamentos de monitoramento, bem como a sua localização nas redes de maneira a captar o máximo de informação, constitui uma tarefa complexa, uma vez que as intrusões de contaminantes ou poluentes podem ocorrer em qualquer ponto do sistema e a qualquer momento.

Não existe um procedimento formal ou guias que sirvam de suporte ao estabelecimento da rede de monitoramento de qualidade da água em Sistemas de Distribuição de Água no caso de uma intrusão intencional. Essa lacuna deve-se, principalmente, às incertezas quanto: (a) à avaliação quantitativa da eficiência da localização dos equipamentos de monitoramento e (b) aos problemas clássicos de redes de distribuição de água, como por exemplo, as variações horárias, diárias e sazonais de consumos, além dos aspectos políticos institucionais.

Além disso, modelos explícitos de decaimento ou diluição de substâncias no interior das redes podem ser necessários para o estudo da localização destes equipamentos de forma a simular o deslocamento destas substâncias, tornando o estudo complexo e muitas vezes limitado. O comportamento de substâncias conservativas e substâncias não-conservativas como o cloro no interior das redes de distribuição de água para abastecimento pode ser simulado através de modelos dinâmicos de qualidade da água tais como o EPANET (ROSSMAN, 2000). Observa-se que substâncias como sais e 
nitratos podem rapidamente atingir concentrações aceitáveis através de diluição, antes que possam ser detectados.

\subsection{Objetivo}

O presente trabalho de pesquisa visa estudar o monitoramento das redes de distribuição para detecção de injeção intencional de contaminantes em concentrações e/ou quantidades suficientes para que se propaguem nas direções do fluxo da água no interior das redes. Diferentemente das propostas da maioria dos trabalhos já existentes na literatura, este trabalho visa à detecção da intrusão intencional de contaminantes, do ponto de vista de objetivos múltiplos a serem identificados e investigados.

Mais especificamente, o estudo tem o propósito de estabelecer o número e a localização otimizados de equipamentos a serem instalados em rede de distribuição de água para detecção de contaminação por intrusões intencionais, cuja fundamentação é apresentada nos capítulos seguintes. 


\section{REVISÃO DA LITERATURA}

A Agência Nacional de Vigilância Sanitária (ANVISA) é o órgão
responsável pela regulamentação que estabelece procedimentos e
responsabilidades relativos ao controle e vigilância da qualidade da água para consumo humano no Brasil, através da Portaria n..$^{\circ}$ 518. A ANVISA tomou por base as leis da Agência de Proteção do Meio-Ambiente dos Estados Unidos (U.S. EPA), para elaborar diretrizes em que a Rede de Distribuição de Água (RDA) deve ter a qualidade da sua água monitorada para que se tenha a certeza de que a água distribuída é realmente segura para o consumo da população. A Portaria n. 518 é de uso obrigatório em todo território nacional e algumas de suas exigências são:

- Promover e acompanhar a vigilância da qualidade da água (Art. $5^{\circ}$ );

- Cabe aos responsáveis pela operação do sistema de abastecimento de água, exercer o controle da qualidade da água (Art. $8^{\circ}$ );

- Enquadrar-se dentro do padrão microbiológico de potabilidade (Art. $11^{\circ}$ ), que especifica parâmetros para:

o Água para consumo humano;

o Água na saída do tratamento;

o Água tratada no sistema de distribuição.

- Após a desinfecção, a água deve conter um teor mínimo de cloro residual livre de $0,5 \mathrm{mg} / \mathrm{L}$, sendo obrigatória a manutenção de, no mínimo, 0,2 mg/L em qualquer ponto da rede de distribuição (Art. $\left.13^{\circ}\right)$

- Enquadrar-se dentro do padrão de aceitação de consumo e não ultrapassar o teor máximo de cloro residual livre de 2,0 mg/L (Art. $\left.16^{\circ}\right)$. 
É certo que existem vários parâmetros de qualidade da água, que devem ser analisados e quantificados. O padrão de potabilidade envolve parâmetros químicos, físicos, biológicos e de radioatividade para a água que servirá ao consumo humano.

O padrão microbiológico é adotado para indicar possíveis contaminações por coliformes. A amostragem deve ser feita em pontos distintos: na água da fonte, na saída do tratamento e no sistema de distribuição (reservatórios e rede). No caso dos parâmetros químicos, são analisadas substâncias que possam apresentar riscos à saúde. A Tabela 2.1 mostra os valores máximos permitidos nas análises de qualidade da água.

Tabela 2.1 - Padrão de Potabilidade para algumas substâncias químicas que apresentam risco à saúde.

\begin{tabular}{|c|c|c|c|c|c|}
\hline Parâmetro & Unidade & VMP $^{(1)}$ & Parâmetro & Unidade & VMP $^{(1)}$ \\
\hline \multicolumn{3}{|c|}{ Inorgânicas } & \multicolumn{3}{|c|}{ Orgânicas } \\
\hline Antimônio & $\mathrm{mg} / \mathrm{L}$ & 0,005 & Acrilamina & $\mu \mathrm{g} / \mathrm{L}$ & 0,5 \\
\hline Arsênio & $\mathrm{mg} / \mathrm{L}$ & 0,01 & Benzeno & $\mu \mathrm{g} / \mathrm{L}$ & 5 \\
\hline Bário & $\mathrm{mg} / \mathrm{L}$ & 0,7 & Benzo[a]pireno & $\mu \mathrm{g} / \mathrm{L}$ & 0,7 \\
\hline Cádmio & $\mathrm{mg} / \mathrm{L}$ & 0,005 & Cloreto de Vinila & $\mu \mathrm{g} / \mathrm{L}$ & 5 \\
\hline Cianeto & $\mathrm{mg} / \mathrm{L}$ & 0,07 & 1,2 dicloetano & $\mu \mathrm{g} / \mathrm{L}$ & 10 \\
\hline Chumbo & $\mathrm{mg} / \mathrm{L}$ & 0,01 & 1,1 dicloeteno & $\mu \mathrm{g} / \mathrm{L}$ & 30 \\
\hline Cobre & $\mathrm{mg} / \mathrm{L}$ & 2 & Diclometano & $\mu \mathrm{g} / \mathrm{L}$ & 20 \\
\hline Cromo & $\mathrm{mg} / \mathrm{L}$ & 0,05 & Estireno & $\mu \mathrm{g} / \mathrm{L}$ & 20 \\
\hline Fluoreto & $\mathrm{mg} / \mathrm{L}$ & 1,5 & Tetracloreto de carbono & $\mu \mathrm{g} / \mathrm{L}$ & 2 \\
\hline Mercúrio & $\mathrm{mg} / \mathrm{L}$ & 0,001 & Tetracloroeteno & $\mu \mathrm{g} / \mathrm{L}$ & 40 \\
\hline Nitrato & $\mathrm{mg} / \mathrm{L}$ & 10 & Triclorobenzeno & $\mu \mathrm{g} / \mathrm{L}$ & 20 \\
\hline Nitrito & $\mathrm{mg} / \mathrm{L}$ & 1 & \begin{tabular}{|l|} 
Tricloroeteno \\
\end{tabular} & $\mu \mathrm{g} / \mathrm{L}$ & 70 \\
\hline Selênio & $\mathrm{mg} / \mathrm{L}$ & 0,01 & & & \\
\hline \multicolumn{3}{|c|}{ Agrotóxicos } & \multicolumn{3}{|c|}{ Desinfetantes e secundários } \\
\hline$\overline{\text { Alaclor }}$ & 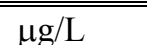 & 20 & Bromato & $\overline{\mathrm{mg} / \mathrm{L}}$ & 0,025 \\
\hline Aldrin & $\mu \mathrm{g} / \mathrm{L}$ & 0,03 & Clorito & $\mathrm{mg} / \mathrm{L}$ & 0,2 \\
\hline Atrazina & $\mu \mathrm{g} / \mathrm{L}$ & 2 & Cloro Livre & $\mathrm{mg} / \mathrm{L}$ & 5 \\
\hline Bentazona & $\mu \mathrm{g} / \mathrm{L}$ & 300 & Monocloroamina & $\mathrm{mg} / \mathrm{L}$ & 3 \\
\hline Clordano & $\mu \mathrm{g} / \mathrm{L}$ & 0,2 & 2,4,6 triclorofenol & $\mathrm{mg} / \mathrm{L}$ & 0,2 \\
\hline Hexaclorobenzeno & $\mu \mathrm{g} / \mathrm{L}$ & 1 & Trihalometanos total & $\mathrm{mg} / \mathrm{L}$ & 0,1 \\
\hline Metolacloro & $\mu \mathrm{g} / \mathrm{L}$ & 10 & & & \\
\hline Pentaclorofenol & $\mu \mathrm{g} / \mathrm{L}$ & 9 & \multicolumn{3}{|c|}{ Cianotoxinas } \\
\hline Permetrina & $\mu \mathrm{g} / \mathrm{L}$ & 20 & Microcistinas & $\mu \mathrm{g} / \mathrm{L}$ & 1 \\
\hline
\end{tabular}

${ }^{(1)}$ Valor máximo permitido. Fonte: Portaria 518/MS 
Os parâmetros físicos e de radioatividade complementam os apresentados, garantindo um fornecimento de água potável dentro dos níveis de aceitação e com a qualidade necessária. Para efetuar as análises destes parâmetros, os responsáveis pelo controle da qualidade da água do sistema de abastecimento, devem elaborar e aprovar, junto à autoridade pública, um plano de amostragem, garantindo que seja realizado um mínimo de análises estipulado pela Portaria 518/MS. As Tabelas 2.2 e 2.3 descrevem o número e a freqüência de amostragem necessárias para mananciais superficiais, em função do ponto de amostragem e do número da população a ser abastecida.

Tabela 2.2 - Número mínimo de amostragens, em mananciais superficiais, para fins de análises físicas, químicas e de radioatividade, em função do plano de amostragem e da

\begin{tabular}{|c|c|c|c|c|}
\hline \multirow{2}{*}{ Parâmetro } & \multirow{2}{*}{$\begin{array}{c}\text { Saída do } \\
\text { tratamento } \\
\left(n^{\circ} \text { de }\right. \\
\text { amostras por } \\
\text { unidade de } \\
\text { tratamento) }\end{array}$} & \multicolumn{3}{|c|}{$\begin{array}{c}\begin{array}{c}\text { Sistema de distribuição } \\
\text { (reservatórios e rede) }\end{array} \\
\text { População Abastecida }\end{array}$} \\
\hline & & $\begin{array}{l}<50.000 \\
\text { hab. }\end{array}$ & $\begin{array}{c}50.000 \text { a } 250.000 \\
\text { hab. }\end{array}$ & $\begin{array}{c}>250.000 \\
\text { hab. }\end{array}$ \\
\hline Cor / pH /Turbidez & 1 & 10 & $1 \mathrm{p} /$ cada $5.000 \mathrm{hab}$ & $40+1 \mathrm{p} /$ cada $25.000 \mathrm{hab}$ \\
\hline Cloro residual livre & 1 & \\
\hline Fluoreto & 1 & 5 & $1 \mathrm{p} /$ cada $10.000 \mathrm{hab}$ & $20+1 \mathrm{p} /$ cada $50.000 \mathrm{hab}$ \\
\hline Cianotoxinas & 1 & \multicolumn{3}{|c|}{$(2)$} \\
\hline Trihalometanos & 1 & 1 & 4 & 4 \\
\hline Demais parâmetros & 1 & 1 & 1 & 1 \\
\hline
\end{tabular}

(1) - Em todas as amostras para análises microbiológicas deve ser efetuada, no momento da coleta, medição de cloro residual livre ou de outro composto residual ativo, caso o agente desinfetante não seja o cloro.

(2) - Sempre que o número de cianobactérias na água do manancial, no ponto de captação, exceder 20.000 células/ml durante o monitoramento, será exigida análise semanal de cianotoxinas na água de saída do tratamento e nas entradas (hidrômetros) das clinicas de hemodiálise e indústria de injetáveis, sendo que esta análise pode ser dispensada quando não houver comprovação de toxidade na água bruta por meio da realização semanal de bioensaios em camundongos. 
Tabela 2.3 - Freqüência mínima de amostragem para o controle da qualidade da água de sistema de abastecimento, para fins de análises físicas, químicas e de radioatividade, em função do plano de amostragem e da população abastecida em mananciais superficiais.

\begin{tabular}{|c|c|c|c|c|}
\hline \multirow{3}{*}{ Parâmetro } & \multirow{3}{*}{$\begin{array}{c}\text { Saída do } \\
\text { tratamento } \\
\text { (freqüência } \\
\text { por unidade } \\
\text { de } \\
\text { tratamento) }\end{array}$} & \multicolumn{3}{|c|}{$\begin{array}{c}\text { Sistema de distribuição } \\
\text { (reservatórios e rede) }\end{array}$} \\
\hline & & \multicolumn{3}{|c|}{ População Abastecida } \\
\hline & & $\begin{array}{l}<50.000 \\
\text { hab. }\end{array}$ & $\begin{array}{c}50.000 \text { a } 250.000 \\
\text { hab. }\end{array}$ & $\begin{array}{c}250.000 \\
\text { hab. }\end{array}$ \\
\hline Cor / pH /Turbidez & a cada 2 horas & mensal & mensal & mensal \\
\hline Cloro residual livre & a cada 2 horas & \multicolumn{3}{|c|}{ (1) } \\
\hline Cianotoxinas & semanal & \multicolumn{3}{|c|}{ (2) } \\
\hline Trihalometanos & trimestral & trimestral & trimestral & trimestral \\
\hline Demais parâmetros & semestral & semestral & semestral & semestral \\
\hline Coliformes totais $^{(3)}$ & - & \multicolumn{2}{|c|}{$\begin{array}{l}\text {-até 5.000: } 10 . \\
\text {-de } 5.000 \text { a } 20.000 \text { hab: } 1 \text { p/ cada } 5000 \text { hab. } \\
\text { - de } 20.000 \text { a } 250.000 \text { hab: } 30+1 \text { p/ cada } \\
2.000 \text { hab. }\end{array}$} & $\begin{array}{l}105+1 \mathrm{p} / \text { cada } \\
5.000 \text { hab, com o } \\
\text { máximo de } 1.000\end{array}$ \\
\hline
\end{tabular}

(1) - Em todas as amostras para análises microbiológicas deve ser efetuada, no momento da coleta, medição de cloro residual livre ou de outro composto residual ativo, caso o agente desinfetante não seja o cloro.

(2) - Sempre que o número de cianobactérias na água do manancial, no ponto de captação, exceder 20.000 células/ml durante o monitoramento, será exigida análise semanal de cianotoxinas na água de saída do tratamento e nas entradas (hidrômetros) das clinicas de hemodiálise e indústria de injetáveis, sendo que esta análise pode ser dispensada quando não houver comprovação de toxidade na água bruta por meio da realização semanal de bioensaios em camundongos.

(3) - Amostras mensais.

A Portaria 518/MS define também, quais são os pontos ideais de coleta das amostras no sistema de distribuição (reservatório e rede). Segundo ela, devem ser analisados critérios de abrangência espacial e pontos estratégicos, como os:

- próximos a grande circulação de pessoas: terminais rodoviários, terminais ferroviários, etc;

- edifícios que abriguem grupos populacionais de risco: hospitais, creches, asilos, etc;

- localizações em trechos vulneráveis do sistema de distribuição: pontas de rede, pontos de queda de pressão, locais afetados por manobras, sujeitos à intermitência de abastecimento, etc; 
- locais com sistemáticas notificações de agravos à saúde tendo como possíveis causas agentes de veiculação hídrica.

Segundo o Decreto Presidencial 5440/05 do Senado Federal, é assegurado à população o direito de conhecer os resultados das análises que comprovam o padrão de qualidade da água consumida. Este decreto também estabelece que o consumidor deve receber do prestador de serviço de distribuição de água um relatório anual contendo, dentre outras, as seguintes informações:

- resumo dos resultados das análises da qualidade da água distribuída, discriminados mensalmente, mencionando, para cada parâmetro analisado, o valor máximo permitido, o número de amostras realizadas, o número de amostras anômalas detectadas, o número de amostras em conformidade com o plano de amostragem estabelecido em norma do Ministério da Saúde e as medidas adotadas face às anomalias verificadas,

- particularidades próprias da água do manancial ou do sistema de abastecimento, como presença de algas com potencial tóxico, ocorrência de flúor natural, ocorrência sistemática de agrotóxicos no manancial, e as ações corretivas e preventivas que estão sendo adotadas para a sua regularização.

Com vistas ao atendimento dos padrões de qualidade que a água deve obedecer, alguns trabalhos, citados ao decorrer do capítulo, têm sido desenvolvidos com o objetivo de identificação da rede de monitoramento da qualidade da água em redes. Esses trabalhos, geralmente, visam à qualidade da água em termos do cloro residual ou intrusão de contaminantes. 
LEE e DEININGER (1992) enfocaram a localização de estações de monitoramento com base na premissa de que se a qualidade da água em um nó amostrado for boa, então ela será aceitável em um nó imediatamente à montante. Para isso, os referidos autores adotaram o termo covered node no intuito de expressar que a qualidade da água em um nó particular pode ser inferida através da amostragem de alguns nós à jusante. Através do estudo das vazões do sistema para um padrão de demanda, usando simuladores hidráulicos como o Kentucky Pipe Model ou WADISO (GESSLER e WALSKI 1985), construíram a matriz de fração de água, partindo do princípio de que, quando se amostra uma demanda $d$ de um nó de demanda total $D$, a fração $d / D$ da qualidade é conhecida. Adotando-se um critério de valor, como por exemplo $75 \%$, que significa que os valores da fração $d / D$ abaixo deste critério, receberam o valor " 0 ” e os valores da fração $d / D$ acima deste critério receberam o valor "1" na matriz de cobertura. Essa matriz foi utilizada para formular um problema de Programação Inteira (PI) e, assim, localizar estações de monitoramento de maneira a atingir a cobertura máxima.

KUMAR et al. (1997) apontaram que o método anterior tem alto custo computacional sendo de difícil controle no caso de sistemas de grande dimensão em termos do número de nós, tubos, etc. Sugeriram, portanto, modificações na forma de montar a matriz fração de água, visando reduzir o esforço computacional empenhado na resolução do problema. Os dois métodos mencionados anteriormente são mais adequados para o caso da deterioração interna gradual da qualidade da água, por exemplo, da redução do cloro 
residual, uma vez que não levam em consideração o volume de água de má qualidade consumida e o tempo para que ocorra a detecção. Partem da premissa de que se o nó amostrado tem água de boa qualidade, o nó de montante também o terá.

KESSLER et al. (1998) propuseram um método para determinar a localização de estações de monitoramento com objetivo de captar uma intrusões acidentais de contaminação dentro de um determinado nível de serviço, definido como o volume máximo de água contaminada consumida até sua detecção. O método envolve a avaliação de uma rede auxiliar, baseada na teoria dos grafos e descrita nos capítulos posteriores, que representa as direções de fluxo de água possíveis no interior da rede para um ciclo diário de demanda típico, associado a um algoritmo de caminhos mais curtos (FLOYD, 1962) para identificar domínios de poluição. Um algoritmo de conjunto de cobertura é aplicado também para determinar a localização das estações de monitoramento de maneira ótima (CHRISTOFIDES, 1975).

KESSLER et al. (1998) assumiram que: (a) qualquer nó da rede é uma fonte possível de poluição e em qualquer momento, (b) toda água que passa através de um nó poluente é considerada contaminada independentemente da concentração do poluente, (c) a velocidade de transporte do poluente corresponde à velocidade média através da seção transversal do tubo, (d) a intrusão do poluente ocorre de modo contínuo, (e) o sistema de detecção prevê a medida monitorada em tempo real e (f) a probabilidade de cada nó constituir fonte de poluição é a mesma, apesar de cada nó ser considerado como tal a cada 
vez. Dentre as hipóteses mencionadas, verifica-se que a probabilidade de cada nó constituir fonte de poluição pode não ser a mesma, pois sempre haverá nós mais propícios devido a alguns fatores como, por exemplo, a sua localização.

O método foi validado em duas redes da literatura e recebeu críticas de KUMAR et al. (1999) com relação a dois aspectos: (a) a analogia conceitual com estudos anteriores, de LEE e DEININGER (1992) e KUMAR et al. (1997) e (b) a sugestão de um critério alternativo ao nível de serviço em termos do volume consumido para tempo, que OTSFELD e KESSLER (1999) rebateram com convicção, pois defendem que seu estudo é para monitoramento da rede em caso de uma intrusão de contaminação em qualquer ponto da rede e não para verificar a deterioração da qualidade ao longo da rede, como nos estudos anteriores. Já em relação ao nível de serviço, o volume consumido dá noção da magnitude do dano causado mais diretamente.

Observa-se, entretanto, que certa razão pode ser atribuída a KUMAR et al. (1999). Autores como WATSON et al. (2004) defendem que múltiplos objetivos devem ser considerados no projeto da rede de sensores e que sua localização otimizada deve ser considerada de forma a minimizar os custos e maximizar o nível de proteção. Eles consideram os seguintes objetivos:

(1) minimização da população exposta: número de pessoas expostas ao contaminante antes da detecção pelos sensores;

(2) minimização do tempo para detecção: tempo entre a intrusão intencional e detecção do contaminante pelos sensores;

(3) minimização do volume consumido: quantia de água contaminada consumida pela população antes da detecção pelo sensor; 
(4) minimização da extensão da contaminação: comprimento de tubulação contaminado pela intrusão e;

(5) minimização do número de detecções falhas: a proporção de intrusões não detectadas pelos sensores.

Para cada um desses objetivos apresentados, WATSON et al. (2004) formularam um problema de otimização de programação linear inteira mista e examinaram a posição dos sensores para duas redes de distribuição reais. Além disso, analisaram as relações de melhor compromisso entre diversos desses objetivos, concluindo que algoritmos robustos para o problema da localização dos sensores devem considerar simultaneamente objetivos múltiplos.

Problemas de segurança em redes de abastecimento de água podem ser formulados de diversas maneiras, dependendo do objetivo a ser perseguido. Um deles é minimizar o risco de contaminações usando sensores para detecção oportuna. BERRY et al.(2003) propôs um modelo de localização otimizada de sensores visando minimizar custos e maximizar benefícios, usando a teoria de otimização. A formulação linear proposta por BERRY et al. (2003) para o problema define a rede de água como um grafo $\mathrm{G}=(\mathrm{V} ; \mathrm{E})$ onde $\mathrm{E}$ é o conjunto de arestas representativo das tubulações, e V é o conjunto de vértices ou nós de conexão dos tubos (por exemplo: reservatórios, tanques, pontos de consumo, etc.). Uma intrusão intencional é representada por uma única injeção de um grande volume de contaminante em um único ponto da rede. O simulador hidráulico EPANET (ROSSMAN, 2000) é usado para determinar a propagação 
do agente contaminador, o sentido de fluxo e outras variáveis de estado. A formulação de PL proposta por BERRY et al. (2003) é explicitada como:

$$
\begin{array}{lc}
\text { Minimizar } & \sum_{i=1}^{n} \sum_{p=1}^{P} \sum_{j=1}^{n} \alpha_{i p} C_{i p j} \delta_{j p} \\
\text { onde : } & i=1, \cdots, n ; p=1, \cdots, P \\
C_{i p j}=1 & i=1, \cdots, n-1, i<j \\
s_{i j}=s_{i j} & (k, j) \in E ; s . t . f_{k j p}=1 \\
C_{i p j} \geq C_{i p k}-s_{k j} & s_{i j} \in(0,1) ;(i, j) \in E \\
\sum_{(i, j) \in E, 1<j} s_{i j} \leq S_{\max } &
\end{array}
$$

onde: - $n$ é o número de nós da rede;

- $\alpha_{i p}$ a probabilidade de intrusão no nó $v_{i}$, durante um padrão de consumo $p$ do total de padrões $P$;

- $\delta_{j p}$ representa a densidade de população abastecida pelo nó $j$ sob o padrão de consumo $p$;

- $C_{i p j}$ é o indicador de contaminação, sendo igual a 1 se o nó $v_{j}$ é contaminado pela intrusão do nó $v_{i}$, caso contrário é igual a 0 ;

- A variável de decisão $s_{i j}$ indica a existência ou não (1 ou 0 ) de sensor no tubo de conexão dos nós $i$ e $j$;

- $f_{k j p}$ é o parâmetro binário que representa o risco de contaminação.

Observa-se que para o problema formulado, os sensores são localizados nos tubos de conexão e o consumo ocorre nos nós, desta forma evita-se que a contaminação se propague para outro nó, porém não se evita que o consumo do nó fonte de contaminação seja descoberto.

OSTFELD e SALOMONS (2004) desenvolveram uma revisão estado da arte sobre o assunto, tecendo a seguinte crítica a trabalhos como os de LEE e DEININGER (1992), KUMAR et al. (1997), AL-ZAHRANI e MOIED (2001), HARMANT et al. (1999), WOO et al. (2001) e TRYBY e UBER (2001): usam o princípio de que se a qualidade de jusante é aceitável, então a 
água abastecida antes de atingir aquele nó é aceitável. Com isso, a solução ótima tende a indicar pontos de jusante e imediatamente antes de mistura de fluxos. Essa idéia básica é aceitável para substâncias não-conservativas na rede, como o cloro, porém, os pontos de monitoramento podem ficar distantes o suficiente para perder ou retardar a detecção de intrusões até que muitos consumidores tenham sido afetados.

OSTFELD e SALOMONS (2004) realçam duas falhas nos trabalhos de KESSLER e OSTFELD (1998), KESSLER et al. (1998), e OSTFELD e KESSLER (2001) relativas a não levar em consideração variações da qualidade da água com o tempo e seu comportamento na rede de distribuição. Com o objetivo de monitorar a intrusão intencional de contaminantes, propõem um modelo para o qual introduzem a seguinte terminologia:

- Evento de poluição: injeção intencional de um contaminante em um ou mais nós de um sistema de distribuição em dadas ocasiões de determinadas durações e concentrações;

- Nível de serviço: o máximo volume de água poluída exposta ao público a uma concentração superior ao nível mínimo estabelecido;

- Domínio do evento de poluição: conjunto de todos os nós contaminados por um dado evento de poluição e a um nível de serviço, ou seja, conjunto dos nós cuja concentração seja superior ao nível mínimo estabelecido e cujo volume acumulativo corresponda ao volume do nível de serviço;

- Domínio de detecção de um nó: série de todos os eventos de poluição detectados pela estação de monitoramento localizada em um determinado nó; 
- Redundância: número relativo aos eventos de poluição detectados por mais que uma estação de monitoramento.

Assumem que qualquer nó pode se tornar uma fonte de poluição em qualquer momento, espalhando o poluente à jusante por advecção e que o sistema de detecção é capaz de prover informação em tempo real.

O método proposto por OSTFELD e SALOMONS (2004) expande o conceito da matriz de poluição de KESSLER et al. (1998) por admitir cenários aleatórios de injeção múltipla de contaminantes, através da matriz de poluição aleatória. O método compreende duas etapas. Primeiramente, cria-se uma matriz de poluição aleatória. Para tanto, cenários de injeção múltipla de contaminante são construídos aleatoriamente e simulados hidraulicamente para um ciclo diário de demanda com o objetivo de identificar quais nós detectam a contaminação introduzida segundo cada cenário. Dessa maneira, constrói-se a matriz de nós $i$ e cenários $j$ de contaminação cujos elementos $A_{i j}$ assumem o valor 0 ou 1 à medida que o nó $i$ é capaz ou não de detectar a contaminação preconizada pelo cenário $j$, dentro de determinado nível de serviço, neste caso, o máximo volume de água poluída exposta à população. A segunda fase do método consiste em determinar a melhor combinação de $N$ (valor fixo) nós de monitoramento de maneira a detectar o maior número de cenários possível. Os Algoritmos Genéticos são utilizados como uma alternativa ao algoritmo de CHRISTOFIDES (1975), para identificar a melhor combinação de $N$ linhas da matriz aleatória. 
As mesmas redes empregadas por KESSLER et al. (1998) foram analisadas para demonstração do método, que, segundo os autores, requer equipamentos de deteç̧ão on-line mais adequados que os existentes no mercado.

Os autores destacam que o monitoramento de contaminantes e poluentes em tempo real quando combinado com a simulação hidráulica da rede pode desempenhar um papel importante no rastreamento e compreensão da extensão da contaminação em um sistema de distribuição de água. Ressaltam a principal limitação à aplicação do modelo proposto: a hipótese de leitura em tempo real do equipamento de monitoramento, pois atualmente existem no mercado monitores on-line para turbidez, cloro residual, $\mathrm{pH}$, dentre outros, que só servem como indicadores indiretos de níveis de contaminação. Os autores sugerem ainda pesquisas futuras para enfrentar os seguintes tópicos: consideração de injeções de contaminação ao longo da tubulação (não necessariamente através de um sistema de nós); uso de diferentes medidas de nível de serviço; uso de técnicas mais sofisticadas para reduzir o esforço computacional das avaliações. Como conclusão, reforçam a importância do estudo, devido à onda de ataques terroristas, sendo a injeção de contaminantes uma grande ameaça para a sociedade.

Duas críticas podem ser feitas em relação ao método proposto por OSTFELD e SALOMONS (2004). O primeiro diz respeito à técnica de amostragem dos cenários aleatórios de poluição, cuja eficiência deve ser melhor 
investigada, e a segunda diz respeito à necessidade do conhecimento prévio da concentração dos poluentes a priori desconhecida.

DAVIDSON et al. (2005) desenvolveram uma nova abordagem para análise de redes de distribuição de água durante um evento de contaminação, pois modelos computacionais anteriores que prevêem o grau de extensão da contaminação são modelos considerados “demand-driven”. A nova abordagem requer um Supervisory Control and Data Acquisition (SCADA) acoppldo ao modelo. O SCADA recebe dados de vazão e pressão em vários pontos da rede e níveis de água do reservatório em tempo real, que são utilizados para criar matrizes de conectividade que consideram a projeção do pior caso de propagação em potencial da contaminação obtida pela combinação de efeitos para vários possíveis cenários de operação. As matrizes de conectividade são capazes de representar a rede em todas possíveis situações de operação, sendo uma matriz $N \times N$ binária, com $\mathrm{N}$ o número de nós da rede, o valor " 1 " representa a existência de possibilidade de ocorrer o fluxo entre os nós e o valor "0", não. Os autores descrevem dois métodos para criar estas matrizes, um baseado no modo de operação da rede através de dados e o outro em caminhos do fluxo, ou seja, através de simulação. O interessante é que os resultados produzidos pelos dois métodos são iguais, dando confiabilidade ao estudo. A necessidade de estimar demandas e potenciais chances de erro da projeção da extensão da contaminação é eliminada do procedimento, que produz uma projeção da extensão de contaminação mais conservativa que modelos hidráulicos convencionais, e por essa razão mais segura. 


\section{MATERIAIS}

O trabalho de pesquisa aqui desenvolvido é de natureza computacional e como tal empregou os recursos disponíveis no Laboratório de Simulação Numérica (LABSIN) do Departamento de Hidráulica e Saneamento (SHS) da Escola de Engenharia de São Carlos (EESC-USP) listados a seguir.

\subsection{Microcomputador}

As simulações foram realizadas em computadores com a seguinte configuração mínima: processador de $900 \mathrm{MHz}$ e 256 Mbytes de memória RAM.

\subsection{Softwares e ferramentas}

\subsubsection{Builder C++ (Borland)}

O $\mathrm{C}++$ Builder da Borland é um ambiente visual, orientado a objetos que tem por finalidade desenvolver aplicações para o Windows. Usando o $\mathrm{C}++$ Builder, pode-se utilizar diversas bibliotecas de domínio público disponíveis para realizar a simulação hidráulica (EPANET) e otimização via AGs (MOMHLIB++).

\subsubsection{MOMHLIB++ (Jaszkiewicz, 1999) - Algoritmos Genéticos Multiobjetivo}

Biblioteca de domínio público na linguagem $\mathrm{C}++$ possui implementados os Algoritmos Genéticos Multiobjetivo SPEA, NSGA e NSGAII. Apresenta a vantagem de permitir que o usuário implemente os 
operadores genéticos básicos (mutação e recombinação) mais adequados ao problema em mãos.

\subsubsection{Simulador EPANET}

EPANET (ROSSMAN, 2000) é um programa de computador, de domínio público, que permite executar simulações hidráulicas e de parâmetros de qualidade de água em sistemas de transporte e de distribuição de água, capaz de calibrar modelos e visualizar os resultados em vários formatos. Desenvolvido pela U.S. Environmental Protection Agency (U.S. EPA) e amplamente testado e utilizado nas avaliações de redes de distribuição do mundo todo. Através de sua biblioteca dinâmica de funções, o EPANET permite personalizar mecanismos computacionais fazendo uso do simulador para as necessidades específicas de cada problema a ser tratado. As funções implementadas na biblioteca dinâmica são escritas em três linguagens: $\mathrm{C} / \mathrm{C}++$, Delphi Pascal e Visual Basic ou ainda em qualquer linguagem em que as funções possam ser chamadas a partir de um arquivo em formato DLL.

O simulador hidráulico EPANET permite:

- calcular perda de carga utilizando as fórmulas de Hazen-Williams, Darcy-Weisbach ou Chezy-Manning;

- considerar perdas de carga unitária em curvas, alargamentos, estreitamentos, etc.;

- modelar bombas de velocidade constante ou variável;

- calcular a energia de bombeamento e o respectivo custo;

- modelar os principais tipos de válvulas, incluindo válvulas de seccionamento, de retenção, reguladoras de pressão e de vazão; 
- modelar reservatórios de armazenamento de nível variável de formas diversas, através de curvas de volume em função da altura de água;

- considerar múltiplas categorias de consumo nos nós, cada uma com um padrão próprio de variação no tempo;

- modelar relações entre pressão e vazão efluente de dispositivos emissores (por exemplo aspersores de rega, ou consumos dependentes da pressão);

- possibilitar basear as condições de operação do sistema em controles simples, dependentes de uma só condição (por exemplo; altura de água num reservatório de nível variável, tempo), ou em controles com condições múltiplas.

Para modelação da qualidade da água, o EPANET tem a capacidade de:

- avaliar o transporte de um constituinte não-reativo (por exemplo, um traçador) através da rede ao longo do tempo;

- avaliar o transporte, mistura e transformação de um constituinte reativo, à medida que este sofre decaimento (por exemplo, cloro) ou retomada do crescimento (por exemplo, um subproduto da desinfecção) com o tempo;

- identificar o tempo de percurso da água através da rede;

- calcular a porcentagem de vazão que, com origem em determinado nó, atinge qualquer outro nó ao longo do tempo (por exemplo, cálculo da importância relativa de duas origens de água diferentes);

- modelar reações de decaimento do cloro no centro e na parede da tubulação;

- utilizar cinéticas de ordem n para modelar reações no centro das tubulações e reservatórios;

- utilizar cinéticas de ordem 0 ou 1 para modelar reações na parede das tubulações; 
- definir limites para a transferência de massa na modelação de reações na parede das tubulações;

- permitir que as reações de crescimento ou decaimento sejam controladas por um valor de concentração-limite;

- aplicar coeficientes de reação globais, que podem ser modificados individualmente para cada tubulação da rede;

- possibilidade de relacionar o coeficiente de reação na parede com a rugosidade da tubulação;

- definir variação temporal da concentração ou de entrada de massa em qualquer ponto da rede;

- modelar reservatórios de armazenamento de nível variável como reatores de mistura completa, de escoamento em êmbolo ou ainda de mistura com dois compartimentos.

Assim, com o auxílio do EPANET podem-se efetuar os seguintes tipos de análise:

- mistura de água a partir de diversas origens;

- determinação do tempo de percurso da água através de um sistema;

- decaimento de cloro residual;

- determinação do crescimento de subprodutos da desinfecção;

- rastreio da propagação de contaminantes ao longo da rede.

A ferramenta tem como principais funções:

- rodar uma simulação completa, ou seja, simular comportamento hidráulico ou de qualidade da água de uma rede para determinadas características e parâmetros;

- obter informações dos nós da rede (por exemplo, identificação, tipo, valores de demanda, posição geográfica, qualidade, entre outros); 
- obter informações das tubulações da rede (por exemplo, identificação, tipo, valores de diâmetro, comprimento, vazão, perda de carga, velocidade, entre outros);

- obter outras informações da rede como padrões de tempo, números de nós e tubos, entre outros. 


\section{O MÉTODO}

O método aqui proposto compreende:

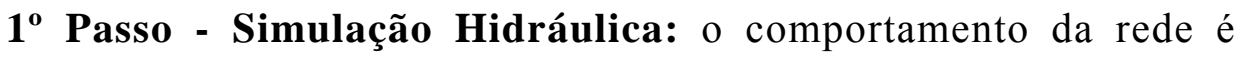
simulado hidraulicamente para intervalos de tempo horários de 24 horas representativo do ciclo de demandas típico de um dia.

$2^{\circ}$ Passo - Construção da rede auxiliar: com base nos resultados das simulações de comportamento da rede para cada intervalo de tempo é construída uma rede auxiliar conforme proposta de KESSLER et al. (1998), em que os tempos de percurso médios são associados a cada trecho (tubo) da rede.

$3^{\circ}$ Passo - Determinação dos caminhos mais curtos: a rede auxiliar é usada para determinação dos caminhos mais curtos que definem os tempos de propagação de um nó fonte de poluição para os outros nós, usando o algoritmo de Floyd.

$4^{\circ}$ Passo - Determinação das matrizes de poluição: as matrizes de poluição são construídas considerando o nível de serviço em termos do volume consumido até a detecção, do tempo transcorrido até a detecção e do máximo espalhamento linear da contaminação até a detecção. Observa-se que tais objetivos foram estabelecidos com base na revisão da literatura, que os colocou em evidência, conforme o item 5.5.1 desta.

$5^{\circ}$ Passo - Determinação dos nós de monitoramento: essa etapa do trabalho representa uma inovação à metodologia proposta por KESSLER et 
Estudo da Localização Otimizada de Equipamentos em Rede de Distribuição de Água para Deteç̧ão de Contaminação

al. (1998). Determinam-se os conjuntos de nós de monitoramento que representam as melhores relações de compromisso entre os objetivos definidos para a resolução do problema usando AGs multiobjetivo. 


\section{METODOLOGIA APLICADA: Rede Net1}

Para facilitar a compreensão do método, utilizou-se inicialmente uma rede, denominada Net1, encontrada no manual do usuário do EPANET e utilizada por KESSLER et al. (1998) e OSTFELD e SALOMONS (2004). A rede Net 1 consiste em: um reservatório de nível constante de $800 \mathrm{ft}$, uma estação elevatória (bomba) e um tanque (nível d’água variável).

\subsection{Simulação Hidráulica}

As equações da continuidade e da conservação da energia caracterizam as condições de equilíbrio hidráulico das redes de distribuição de água para abastecimento em regime permanente de funcionamento.

Considerando uma rede com $N$ nós e $N F$ nós com carga hidráulica fixa, a relação perda de carga x vazão em uma tubulação entre os nós $i$ e $j$ pode ser representada pela seguinte expressão:

$$
H_{i}-H_{j}=h_{i j}=r \cdot Q_{i j}^{n}+m \cdot Q_{i j}^{2}
$$

onde $H$ é a carga hidráulica no nó, $h$ a perda de carga total, $r$ o termo de perda de carga que depende da formulação utilizada, $Q$ a vazão na tubulação, $n$ o expoente da formulação perda de carga x vazão e $m$ o coeficiente de perda de carga localizada.

A conservação de massa nos nós é especificada pela expressão:

$$
\sum_{j \in J_{i}} Q_{i j}-C_{i}=0 \quad \text { para } \quad i=1, \ldots, N
$$


em que $C$ é o consumo no nó $i$ e $J_{i}$ são todos os nós conectados ao nó $i$. Assim, conhecendo a carga hidráulica nos $N F$ nós, obtém-se os valores de pressão e vazão na rede que satisfaçam as equações (6) e (7).

Tais equações constituem basicamente o sistema a ser resolvido pelo simulador hidráulico EPANET para obtenção dos valores de pressão e vazão na rede, através do método gradiente.

\subsubsection{Caracterização das Redes}

O sistema de distribuição de água previsto para estudo deverá ter suas características definidas para dar suporte às avaliações propostas. Algumas destas características são listadas a seguir.

- posição, cota e demanda de cada nó;

- diâmetro e comprimento de cada tubulação;

- padrão de demanda característica;

- posição, cota e dimensões (se necessário) de cada tanque (reservatório de nível variável) e/ou reservatório;

- posição e característica das bombas.

No caso da rede Net1, sua topologia, característica do tanque e o padrão de demanda são definidos a seguir. 


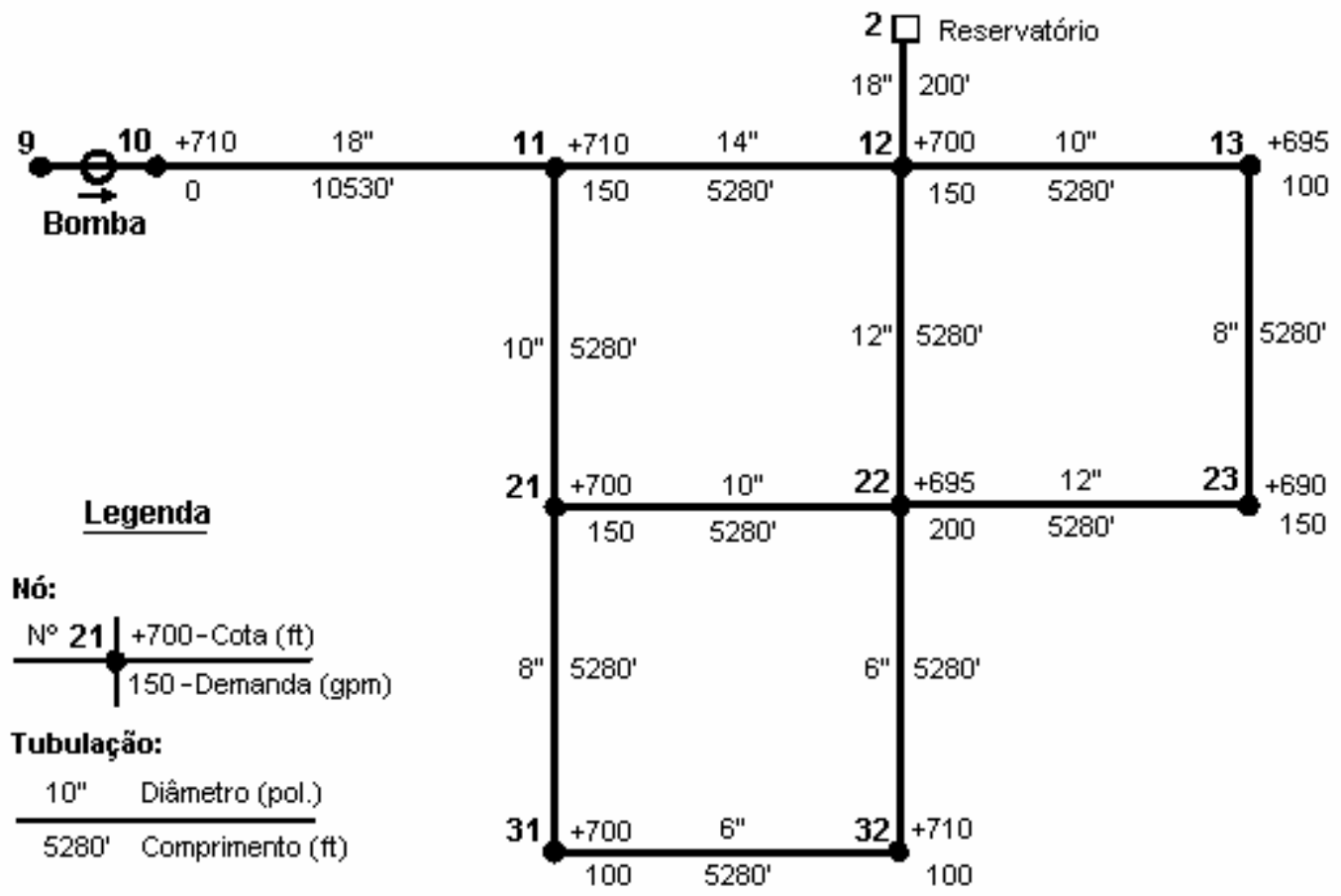

Figura 5.1 - Rede Net1

Tabela 5.1 - Característica do Tanque da rede Net1.

\begin{tabular}{|c|c|c|c|c|c||}
\hline \hline $\begin{array}{c}\text { Número do } \\
\text { Tanque } \\
\text { (Reservatório) }\end{array}$ & $\begin{array}{c}\text { Cota } \\
\text { (ft) }\end{array}$ & $\begin{array}{c}\text { Nível } \\
\text { Inicial } \\
\text { (ft) }\end{array}$ & $\begin{array}{c}\text { Nível } \\
\text { Mínimo } \\
\text { (ft) }\end{array}$ & $\begin{array}{c}\text { Nível } \\
\text { Máximo } \\
\text { (ft) }\end{array}$ & $\begin{array}{c}\text { Diâmetro } \\
\text { (ft) }\end{array}$ \\
\hline \hline 2 & 850 & 120 & 100 & 150 & 50.5 \\
\hline
\end{tabular}

Tabela 5.2 - Características de demanda padrão para 24 horas da rede Net1.

\begin{tabular}{|c|c|}
\hline Hora do dia & Multiplicador da demanda média \\
\hline \hline $24-02$ & 1.0 \\
\hline $02-04$ & 1.2 \\
\hline $04-06$ & 1.4 \\
\hline $06-08$ & 1.6 \\
\hline $08-10$ & 1.4 \\
\hline $10-12$ & 1.2 \\
\hline $12-14$ & 1.0 \\
\hline $14-16$ & 0.8 \\
\hline $16-18$ & 0.6 \\
\hline $18-20$ & 0.4 \\
\hline $20-22$ & 0.6 \\
\hline $22-24$ & 0.8 \\
\hline
\end{tabular}




\subsubsection{Modelagem da Rede}

Definida a rede e suas características, esta deve ser modelada no programa EPANET, para isto deve seguir as seguintes etapas.

- desenhar uma representação esquemática do sistema de distribuição;

- editar as propriedades dos objetos que constituem o sistema;

- descrever as condições de operacionalidade do sistema;

- exportar dados através do menu principal para um arquivo input de formato .INP (Ver Anexo I o arquivo input para a rede Net1).

\subsection{Inicialização do PLEDECORDA_EPA}

Com o suporte do software Builder e da ferramenta do EPANET (ROSSMAN, 2000) para linguagem $\mathrm{C}++$, o programa denominado PLEDECORDA_EPA (Programa de Localização de Equipamentos para DEtecção de COntaminação em Rede de Distribuição de Água - Parte 1 Criação das matrizes de Poluição) importa os dados do arquivo de entrada, que deve ser denominado REDE.INP, ver em Anexo I o arquivo de entrada da Rede Net1. Como resultado, o programa exporta o arquivo PLEDECORDA_EPA.TXT, que contém as velocidades médias dos trechos da rede, as matrizes de Custo (Caminhos mais Curtos) e Roteamento e as três matrizes poluição, uma para cada nível de serviço. Ver Anexo II o arquivo PLEDECORDA_EPA.TXT para a Rede Net1. 


\subsubsection{Parâmetros de Entrada - Níveis de Serviço}

Além do arquivo REDE.INP, o PLEDECORDA_EPA solicita inicialmente os valores limites para os três níveis de serviço a seguir, justificados posteriormente:

(1) Volume Consumido (VC): adotar o valor de 1 a $10 \%$ do volume consumido em um dia pela população. Para a Rede Net1 adotou-se VC $=283.17 \mathrm{~m}^{3}\left(10,000 \mathrm{ft}^{3}\right)$;

(2) Tempo de Detecção (TD): adotar o valor de 1 a 6 horas. Para a Rede Net 1 adotou-se TD $=6$ horas;

(3) Extensão da Contaminação (EC): adotar o valor de 1 a $20 \%$ do comprimento da rede. Para a Rede Net1 adotou-se EC $=15 \%$.

Os valores de referência citados para cada nível de serviço foram estipulados através dos resultados do programa PLEDECORDA_EPA e das bibliografias revisadas no capítulo 2 .

\subsection{Construção da Rede Auxiliar}

A rede auxiliar é construída conforme proposto por KESSLER et al. (1998), auxiliada pela simulação hidráulica para a obtenção das velocidades em todas as tubulações para certo padrão de demandas, ou seja, um ciclo de demanda típico (24 horas, em geral). A partir desses dados são construídas duas redes auxiliares, uma para tempo de viagem e outra para extensão de viagem, que consistem de uma série de nós conectados por arcos. Os nós correspondem aos mesmos nós da rede original e para cada trecho ij: (a) um único arco é introduzido ligando os nós $i$ e $j$ se existir tubulação entre esses nós e o fluxo 
ocorre apenas em um sentido e (b) dois arcos paralelos são introduzidos se existir tubulação entre o nó $i$ e o nó $j$ e o fluxo ocorre em ambos sentidos. Para a rede auxiliar representativa do tempo de viagem (ver figura 5.3), atribui-se a cada arco um valor denominado "tempo médio de viagem" ( $L / V)$ que é o comprimento do tubo $(L)$ dividido pela velocidade média $(V)$ do fluxo em cada sentido para o período de simulação (no caso, 24 horas); e para a rede auxiliar representativa da extensão da viagem (ver figura 5.4), atribui-se a cada arco o respectivo comprimento do tubo, considerados porém apenas nos sentidos em que a água flui. Portanto, cada arco contém as informações que possibilitam a determinação do domínio de cada nó.

\subsection{Determinação dos Caminhos Mais Curtos}

Assumindo que as partículas de contaminante são conservativas, comportando-se como traçadores e movendo-se com velocidade igual ao fluxo, o tempo de propagação do poluente, do nó $i$ ao nó $j$, corresponde ao tempo mínimo de viagem entre estes nós.

Portanto, deve-se utilizar um algoritmo para definição dos caminhos mais curtos. Existem vários algoritmos e problemas considerados “clássicos" na Teoria dos Grafos. Um desses problemas é o de encontrar um caminho mínimo entre dois vértices. Existem vários algoritmos que resolvem esse problema. Um deles é o algoritmo de Floyd.

\subsubsection{Algoritmo de Floyd (1962)}

Inicialmente, esse algoritmo determina uma Matriz de Custo (Matriz C) representativa do grafo, ou seja, verifica o custo entre cada par de 
vértices. Se existir uma aresta ligando o par de vértices $i$ e $j$, assume-se que o elemento $c_{i j}$ da matriz representa o custo da aresta. Se não existir uma aresta entre o par de vértices, o elemento assume um valor alto $(\infty)$. Entende-se que vértices são os nós da rede, arestas são os tubos e o valor $\infty$ adotado nos estudos é de 888 .

Em seguida, o algoritmo verifica se existe um caminho de menor custo entre cada par de vértices, ao passar por um vértice intermediário. Suponha um grafo com 5 vértices. De um modo geral, após montar a matriz de custo, 5 iterações são necessárias:

- $1^{a}$. Iteração: verificar a existência de caminhos menores passando pelo vértice 1 ;

- 2a . Iteração: verificar a existência de caminhos menores passando pelo vértice 2 ;

- $3^{a}$. Iteração: verificar a existência de caminhos menores passando pelo vértice 3;

- 4a . Iteração: verificar a existência de caminhos menores passando pelo vértice 4;

- 5a Iteração: verificar a existência de caminhos menores passando pelo vértice 5 .

\section{O algoritmo:}

Parte 1 - Inicializar as matrizes de Custo (Matriz C) e de Roteamento (Matriz R).

$$
\mathrm{C}^{0}{ }_{\mathrm{ij}}=\left\{\begin{array}{l}
0, \text { se } i=j \\
\infty, \text { se não existe aresta entre os vértices } i \text { e } j \\
\text { custo da aresta entre os vértices } i \text { e } j
\end{array}\right.
$$




$$
\mathrm{R}_{\mathrm{ij}}^{0}=\left\{\begin{array}{l}
j, \text { se } c_{i j}<\infty \\
0, \text { em caso contrário }
\end{array}\right.
$$

Parte 2 - Verificar, para cada posição da matriz, se existe um caminho mais curto. Considerando que n é o número de vértices, a construção de tais matrizes pode ser feita de acordo com o "pseudo" código:

$$
\begin{aligned}
& \text { Para } k:=1 \text { a n faça } \\
& \text { Para } i:=1 \text { a n faça } \\
& \text { Para } j:=1 \text { a n faça } \\
& \text { Se }\left(C^{k-1}{ }_{i k}+C^{k-1}{ }_{k j}<C^{k-1} i j\right) \\
& C^{k}{ }_{i j}:=C^{k-1}{ }_{i k}+C^{k-1}{ }_{k j} ; \\
& R_{i j}^{k}:=R^{k-1}{ }_{i k} ;
\end{aligned}
$$

Por exemplo, considerando o grafo abaixo, aplica-se o algoritmo de Floyd para encontrar a matriz de custo mínimo.

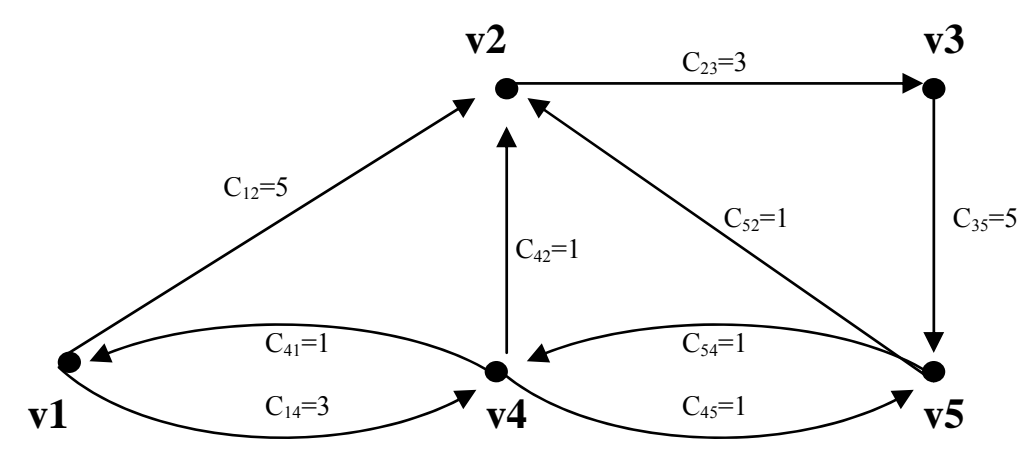

Figura 5.2 - Grafo exemplo 
O primeiro passo, construir as matrizes de custo e roteamento:

Matriz C
\begin{tabular}{l|c|c|c|c|c|}
\multicolumn{1}{c}{ v1 } & v2 & v3 & \multicolumn{1}{c}{ v4 } \\
v1 & 0 & 5 & $\infty$ & 3 & $\infty$ \\
\cline { 2 - 6 } v2 & $\infty$ & 0 & 3 & $\infty$ & $\infty$ \\
\cline { 2 - 6 } v3 & $\infty$ & $\infty$ & 0 & $\infty$ & 5 \\
\cline { 2 - 6 } v4 & 1 & 1 & $\infty$ & 0 & 1 \\
\cline { 2 - 6 } v5 & $\infty$ & 1 & $\infty$ & 1 & 0 \\
\cline { 2 - 6 }
\end{tabular}

\begin{tabular}{|c|c|c|c|c|c|}
\hline & \multicolumn{5}{|c|}{ Matriz $\mathrm{R}^{0}$} \\
\hline & v1 & v2 & v3 & v4 & v5 \\
\hline & 1 & 2 & 0 & 4 & 0 \\
\hline & 0 & 2 & 3 & 0 & 0 \\
\hline & 0 & 0 & 3 & 0 & 5 \\
\hline & 1 & 2 & 0 & 4 & 5 \\
\hline & 0 & 2 & 0 & 4 & 5 \\
\hline
\end{tabular}

A seguir, várias iterações são realizadas para verificar se existe um caminho mais curto entre cada pares de vértices. No início têm-se:

$$
\begin{array}{ll}
\mathrm{K}=1, \mathrm{i}=1, \mathrm{j}=1 & \text { se } \mathrm{C}^{0} 11+\mathrm{C}^{0} 11<\mathrm{C}^{0} 11 ? 0+0<0 ? \quad \text { Não! } \\
\mathrm{K}=1, \mathrm{i}=1, \mathrm{j}=2 & \text { se } \mathrm{C}^{0} 11+\mathrm{C}^{0} 12<\mathrm{C}^{0} 12 ? 0+5<5 ? \text { Não! } \\
\mathrm{K}=1, \mathrm{i}=1, \mathrm{j}=3 & \text { se } \mathrm{C}^{0} 11+\mathrm{C}^{0} 13<\mathrm{C}^{0} 13 ? 0+\infty<\infty \text { ? Não! } \\
\mathrm{K}=1, \mathrm{i}=1, \mathrm{j}=4 & \text { se } \mathrm{C}^{0} 11+\mathrm{C}^{0} 14<\mathrm{C}^{0} 14 ? 0+3<3 ? \text { Não! } \\
\mathrm{K}=1, \mathrm{i}=1, \mathrm{j}=5 & \text { se } \mathrm{C}^{0} 11+\mathrm{C}^{0} 15<\mathrm{C}^{0} 15 ? 0+\infty<\infty \text { ? Não! }
\end{array}
$$

Basicamente, o que se investigou foi se para ir do vértice 1 para qualquer outro, o caminho ficou mais curto ao passar pelo vértice $1(\mathbf{k}=\mathbf{1})$.

Após $j$ atingir $n$, que é igual a 5 (número de vértices), a execução sai do laço de $j$ e $i$ é incrementado para 2. Têm-se:

$$
\begin{array}{lll}
\mathrm{K}=1, \mathrm{i}=2, \mathrm{j}=1 & \text { se } \mathrm{C}^{0} 21+\mathrm{C}^{0} 11<\mathrm{C}^{0} 21 ? \infty+0<\infty \text { ? Não! } \\
\mathrm{K}=1, \mathrm{i}=2, \mathrm{j}=2 & \text { se } \mathrm{C}^{0} 21+\mathrm{C}^{0} 12<\mathrm{C}^{0} 22 ? \infty+5<0 \text { ? Não! } \\
\mathrm{K}=1, \mathrm{i}=2, \mathrm{j}=3 & \text { se } \mathrm{C}^{0} 21+\mathrm{C}^{0} 13<\mathrm{C}^{0} 23 ? \infty+\infty<3 ? \text { Não! } \\
\mathrm{K}=1, \mathrm{i}=2, \mathrm{j}=4 & \text { se } \mathrm{C}^{0} 21+\mathrm{C}^{0} 14<\mathrm{C}^{0} 24 ? \infty+3<\infty \text { ? Não! } \\
\mathrm{K}=1, \mathrm{i}=2, \mathrm{j}=5 & \text { se } \mathrm{C}^{0} 21+\mathrm{C}^{0} 15<\mathrm{C}^{0} 25 ? \infty+\infty<\infty \text { ? Não! }
\end{array}
$$

Demonstrou-se também que para ir do vértice v2 a qualquer outro vértice, o caminho não é encurtado ao passar pelo vértice 1. Em outras palavras, verificou-se que utilizar o vértice 1 como intermediário não encurta o caminho do vértice v2 a qualquer outro. 
Continuando então, $i$ é incrementado para 3:

$\begin{array}{lll}K=1, i=3, j=1 & \text { se } C^{0} 31+C^{0} 11<C^{0} 31 ? \infty+0<\infty ? & \text { Não! } \\ K=1, i=3, j=2 & \text { se } C^{0} 31+C^{0} 12<C^{0} 32 ? \infty+5<\infty ? & \text { Não! } \\ K=1, i=3, j=3 & \text { se } C^{0} 31+C^{0} 13<C^{0} 33 ? \infty+\infty<0 ? & \text { Não! } \\ K=1, i=3, j=4 & \text { se } C^{0} 31+C^{0} 14<C^{0} 34 ? \infty+3<\infty ? & \text { Não! } \\ K=1, i=3, j=5 & \text { se } C^{0} 31+C^{0} 15<C^{0} 35 ? \infty+\infty<5 ? & \text { Não! }\end{array}$

Continuando, $i$ é incrementado para 4:

$\begin{array}{lll}\mathrm{K}=1, \mathrm{i}=4, \mathrm{j}=1 & \text { se } \mathrm{C}^{0} 41+\mathrm{C}^{0} 11<\mathrm{C}^{0} 41 ? 1+0<1 ? & \text { Não! } \\ \mathrm{K}=1, \mathrm{i}=4, \mathrm{j}=2 & \text { se } \mathrm{C}^{0} 41+\mathrm{C}^{0} 12<\mathrm{C}^{0} 42 ? 1+5<1 ? & \text { Não! } \\ \mathrm{K}=1, \mathrm{i}=4, \mathrm{j}=3 & \text { se } \mathrm{C}^{0} 41+\mathrm{C}^{0} 13<\mathrm{C}^{0} 43 ? 1+\infty<\infty ? & \text { Não! } \\ \mathrm{K}=1, \mathrm{i}=4, \mathrm{j}=4 & \text { se } \mathrm{C}^{0} 41+\mathrm{C}^{0} 14<\mathrm{C}^{0} 44 ? 1+3<0 ? & \text { Não! } \\ \mathrm{K}=1, \mathrm{i}=4, \mathrm{j}=5 & \text { se } C^{0} 41+\mathrm{C}^{0} 15<\mathrm{C}^{0} 45 ? 1+\infty<1 ? & \text { Não! }\end{array}$

Continuando, $i$ é incrementado para 5:

$\begin{array}{lll}K=1, i=5, j=1 & \text { se } C^{0} 51+C^{0} 11<C^{0} 51 ? \infty+0<\infty ? & \text { Não! } \\ K=1, i=5, j=2 & \text { se } C^{0} 51+C^{0} 12<C^{0} 52 ? \infty+5<1 ? & \text { Não! } \\ K=1, i=5, j=3 & \text { se } C^{0} 51+C^{0} 13<C^{0} 53 ? \infty+\infty<\infty ? & \text { Não! } \\ K=1, i=5, j=4 & \text { se } C^{0} 51+C^{0} 14<C^{0} 54 ? \infty+3<1 ? & \text { Não! } \\ K=1, i=5, j=5 & \text { se } C^{0} 51+C^{0} 15<C^{0} 55 ? \infty+\infty<0 ? & \text { Não! }\end{array}$

Após $i$ atingir o valor $n$, ou seja, $\mathrm{i}=5$, o laço do $i$ chega ao fim e a variável $k$ é incrementada e assume o valor 2. Investiga-se agora se no percurso entre cada par de vértices o uso do vértice v2 como intermediário torna o caminho mais curto.

$$
\begin{array}{lll}
\mathrm{K}=2, \mathrm{i}=1, \mathrm{j}=1 & \text { se } \mathrm{C}^{0} 12+\mathrm{C}^{0} 21<\mathrm{C}^{0} 11 ? 5+\infty<0 \text { ? } & \text { Não! } \\
\mathrm{K}=2, \mathrm{i}=1, \mathrm{j}=2 & \text { se } \mathrm{C}^{0} 12+\mathrm{C}^{0} 22<\mathrm{C}^{0} 12 ? 5+0<5 ? & \text { Não! } \\
\mathrm{K}=2, \mathrm{i}=1, \mathrm{j}=3 & \text { se } \mathrm{C}^{0} 12+\mathrm{C}^{0} 23<\mathrm{C}^{0} 13 ? 5+3<\infty \text { ? } & \text { Sim!!! } \\
\mathrm{K}=2, \mathrm{i}=1, \mathrm{j}=4 & \text { se } \mathrm{C}^{0} 12+\mathrm{C}^{0} 24<\mathrm{C}^{0} 14 ? 5+\infty<3 \text { ? Não! } \\
\mathrm{K}=2, \mathrm{i}=1, \mathrm{j}=5 & \text { se } \mathrm{C}^{0} 12+\mathrm{C}^{0} 25<\mathrm{C}^{0} 15 ? 5+\infty<\infty \text { ? Não! }
\end{array}
$$

Quando um caminho mais curto é identificado, gera-se a matriz $\mathrm{C}^{1}$, sobrescrevendo-se os valores anteriores. Sendo assim, as matrizes de custo e roteamento atualizadas são: 


\begin{tabular}{l|c|c|c|c|c|}
\multicolumn{7}{c}{ Matriz C } \\
\multicolumn{1}{|c|}{ v1 } & \multicolumn{1}{c}{ v2 } & v3 & \multicolumn{1}{c}{ v4 } \\
v1 & 0 & 5 & $\boldsymbol{8}$ & 3 & $\infty$ \\
\cline { 2 - 6 } v2 & $\infty$ & 0 & 3 & $\infty$ & $\infty$ \\
\cline { 2 - 6 } v3 & $\infty$ & $\infty$ & 0 & $\infty$ & 5 \\
\cline { 2 - 6 } v4 & 1 & 1 & $\infty$ & 0 & 1 \\
\cline { 2 - 6 } v5 & $\infty$ & 1 & $\infty$ & 1 & 0 \\
\cline { 2 - 6 }
\end{tabular}

\begin{tabular}{c|c|c|c|c|c|}
\multicolumn{7}{c}{ Matriz R $^{1}$} \\
\multicolumn{1}{c}{} & \multicolumn{1}{c}{ v1 } & v2 & v3 & v4 & v5 \\
\cline { 2 - 6 } v1 & 1 & 2 & 2 & 4 & 0 \\
\cline { 2 - 6 } v2 & 0 & 2 & 3 & 0 & 0 \\
\cline { 2 - 6 } v3 & 0 & 0 & 3 & 0 & 5 \\
\cline { 2 - 6 } v4 & 1 & 2 & 0 & 4 & 5 \\
\cline { 2 - 6 } v5 & 0 & 2 & 0 & 4 & 5 \\
\cline { 2 - 6 } & & & &
\end{tabular}

Repara-se que a matriz de roteamento agora indica que, para ir do vértice v1 para o vértice v3, é preciso primeiro passar pelo vértice v2.

Repetindo então o procedimento para $\mathrm{k}=2, \mathrm{k}=3, \mathrm{k}=4$ e $\mathrm{k}=5$, no final ter-se-á as duas matrizes:

Matriz $C^{5}$ (custo mínimo)
\begin{tabular}{|c|c|c|c|c|c|} 
v1 & v2 & v3 & v4 & v 5 \\
v1 & 0 & $\mathbf{4}$ & $\mathbf{7}$ & $\mathbf{3}$ & $\mathbf{4}$ \\
\hline v2 & $\mathbf{1 0}$ & 0 & 3 & $\mathbf{9}$ & $\mathbf{8}$ \\
\hline v3 & $\mathbf{7}$ & $\mathbf{6}$ & 0 & $\mathbf{6}$ & 5 \\
\cline { 2 - 6 } v4 & 1 & 1 & $\mathbf{4}$ & 0 & 1 \\
\cline { 2 - 6 } v5 & $\mathbf{2}$ & 1 & $\mathbf{4}$ & 1 & 0 \\
\cline { 2 - 6 }
\end{tabular}

Matriz R 5 (roteamento)
\begin{tabular}{l|c|c|c|c|c|} 
v1 & v2 & v3 & v & v \\
\cline { 2 - 6 } v1 & 1 & $\mathbf{4}$ & $\mathbf{4}$ & 4 & $\mathbf{4}$ \\
v2 & 3 & 2 & 3 & 3 & 3 \\
v3 & 5 & 5 & 3 & 5 & 5 \\
\cline { 2 - 6 } v4 & 1 & 2 & 2 & 4 & 5 \\
v5 & 4 & 2 & 2 & 4 & 5 \\
\cline { 2 - 6 }
\end{tabular}

Assim, a Matriz $\mathrm{C}^{5}$ indica qual será o custo mínimo para ir de um vértice a outro, enquanto a Matriz $\mathrm{R}^{5}$ mostra qual é o caminho que deverá ser percorrido para ir de um vértice a outro com o custo mínimo. Por exemplo:

- Para ir do vértice v1 para o vértice v2, o custo é de valor 4 conforme elemento $\mathrm{c}_{12}$ da Matriz $\mathrm{C}^{5}$. O caminho mais curto é identificado pelos elementos da Matriz $\mathrm{R}^{5}$ da seguinte forma: o valor 4 do elemento $\mathrm{r}_{12}$ indica que o vértice v1 representado pela linha 1 deve passar pela linha 4 que representa o vértice v4, então deve-se agora considerar o elemento $\mathrm{r}_{42}$, já que necessita-se agora ir do vértice $\mathrm{v} 4$ para o vértice v2, o valor 2 significa que deve-se passar pela linha 2 da matriz que 
representa o vértice v2, sendo este o vértice de destino. Portanto o roteamento do caminho mais curto é v1-v4-v2.

- Para ir do vértice v1 para o vértice v3, o custo é de valor 7 conforme elemento $\mathrm{c}_{13}$ da Matriz $\mathrm{C}^{5}$. O caminho mais curto é identificado da seguinte forma: o valor 4 do elemento $r_{13}$ indica que o vértice v1 representado pela linha 1 deve passar pela linha 4 que representa o vértice $v 4$, então deve-se considerar o elemento $r_{43}$, já que necessitase agora ir do vértice v4 para o vértice v3, o valor 2 significa que deve-se passar pela linha 2 da matriz que representa o vértice v2, então deve-se considerar o elemento $r_{23}$, o valor 3 significa que devese passar pela linha 3 da matriz que representa o vértice v3, sendo este o vértice de destino. Portanto o roteamento do caminho mais curto é v1-v4-v2-v3.

O algoritmo de Floyd foi implementado para identificar as matrizes de Custo e Roteamento. Tais matrizes obtidas pela rede Net1 são mostradas a seguir como exemplo. Com base nessas matrizes são construídas as redes auxiliares para Tempo de viagem e para Extensão de viagem. A rede auxiliar para Tempo de viagem é utilizada na construção da matrizes poluição de níveis de serviço em termos do volume máximo consumido de água contaminada e tempo máximo para detecção. A rede auxiliar para Extensão de viagem é utilizada na construção da matriz poluição de nível de serviço em termos da extensão máxima da contaminação. 
Estudo da Localização Otimizada de Equipamentos em Rede de Distribuição de Água para Deteç̧ão de Contaminação

Matriz Custo de Tempo de viagem (h) (Caminhos Mais Curtos):

\begin{tabular}{|c|ccccccccccc|}
\hline Nós: & 10 & 11 & 12 & 13 & 21 & 22 & 23 & 31 & 32 & 9 & 2 \\
\hline 10 & 0 & 2.13 & 3.15 & 5.44 & 3.14 & 4.94 & 9.46 & 4.86 & 6.90 & 888 & 3.30 \\
11 & 888 & 0 & 1.02 & 3.31 & 1.01 & 2.81 & 7.33 & 2.73 & 4.77 & 888 & 1.16 \\
12 & 888 & 6.78 & 0 & 2.29 & 7.79 & 1.79 & 6.31 & 9.51 & 3.75 & 888 & 0.14 \\
13 & 888 & 888 & 888 & 0 & 888 & 888 & 4.02 & 888 & 888 & 888 & 888 \\
21 & 888 & 888 & 888 & 888 & 0 & 3.98 & 9.55 & 1.71 & 5.50 & 888 & 888 \\
22 & 888 & 888 & 888 & 888 & 17.63 & 0 & 5.56 & 19.34 & 1.96 & 888 & 888 \\
23 & 888 & 888 & 888 & 888 & 888 & 888 & 0 & 888 & 888 & 888 & 888 \\
31 & 888 & 888 & 888 & 888 & 888 & 888 & 888 & 0 & 3.79 & 888 & 888 \\
32 & 888 & 888 & 888 & 888 & 888 & 888 & 888 & 888 & 0 & 888 & 888 \\
9 & 0 & 2.13 & 3.15 & 5.44 & 3.14 & 4.94 & 9.46 & 4.86 & 6.90 & 0 & 3.30 \\
2 & 888 & 6.92 & 0.14 & 2.42 & 7.93 & 1.93 & 6.45 & 9.65 & 3.89 & 888 & 0 \\
\hline
\end{tabular}

Matriz Custo de Extensão de viagem Final (ft) (Caminhos Mais Curtos):

\begin{tabular}{|c|ccccccccccc|}
\hline Nós: & 10 & 11 & 12 & 13 & 21 & 22 & 23 & 31 & 32 & 9 & 2 \\
\hline 10 & 0 & 10530 & 15810 & 21090 & 15810 & 21090 & 26370 & 21090 & 26370 & 63530 & 16010 \\
11 & 63530 & 0 & 5280 & 10560 & 5280 & 10560 & 15840 & 10560 & 15840 & 63530 & 5480 \\
12 & 63530 & 5280 & 0 & 5280 & 10560 & 5280 & 10560 & 15840 & 10560 & 63530 & 200 \\
13 & 63530 & 63530 & 63530 & 0 & 63530 & 63530 & 5280 & 63530 & 63530 & 63530 & 63530 \\
21 & 63530 & 63530 & 63530 & 63530 & 0 & 5280 & 10560 & 5280 & 10560 & 63530 & 63530 \\
22 & 63530 & 63530 & 63530 & 63530 & 5280 & 0 & 5280 & 10560 & 5280 & 63530 & 63530 \\
23 & 63530 & 63530 & 63530 & 63530 & 63530 & 63530 & 0 & 63530 & 63530 & 63530 & 63530 \\
31 & 63530 & 63530 & 63530 & 63530 & 63530 & 63530 & 63530 & 0 & 5280 & 63530 & 63530 \\
32 & 63530 & 63530 & 63530 & 63530 & 63530 & 63530 & 63530 & 63530 & 0 & 63530 & 63530 \\
9 & 0 & 10530 & 15810 & 21090 & 15810 & 21090 & 26370 & 21090 & 26370 & 0 & 16010 \\
2 & 63530 & 5480 & 200 & 5480 & 10760 & 5480 & 10760 & 16040 & 10760 & 63530 & 0 \\
\hline
\end{tabular}

Matriz Roteamento Final:

\begin{tabular}{|c|ccccccccccc|}
\hline Nós: & 10 & 11 & 12 & 13 & 21 & 22 & 23 & 31 & 32 & 9 & 2 \\
\hline 10 & 1 & 2 & 2 & 2 & 2 & 2 & 2 & 2 & 2 & 0 & 2 \\
11 & 0 & 2 & 3 & 3 & 5 & 3 & 3 & 5 & 3 & 0 & 3 \\
12 & 0 & 2 & 3 & 4 & 2 & 6 & 4 & 2 & 6 & 0 & 11 \\
13 & 0 & 0 & 0 & 4 & 0 & 0 & 7 & 0 & 0 & 0 & 0 \\
21 & 0 & 0 & 0 & 0 & 5 & 6 & 6 & 8 & 8 & 0 & 0 \\
22 & 0 & 0 & 0 & 0 & 5 & 6 & 7 & 5 & 9 & 0 & 0 \\
23 & 0 & 0 & 0 & 0 & 0 & 0 & 7 & 0 & 0 & 0 & 0 \\
31 & 0 & 0 & 0 & 0 & 0 & 0 & 0 & 8 & 9 & 0 & 0 \\
32 & 0 & 0 & 0 & 0 & 0 & 0 & 0 & 0 & 9 & 0 & 0 \\
9 & 1 & 1 & 1 & 1 & 1 & 1 & 1 & 1 & 1 & 10 & 1 \\
2 & 0 & 3 & 3 & 3 & 3 & 3 & 3 & 3 & 3 & 0 & 11 \\
\hline
\end{tabular}




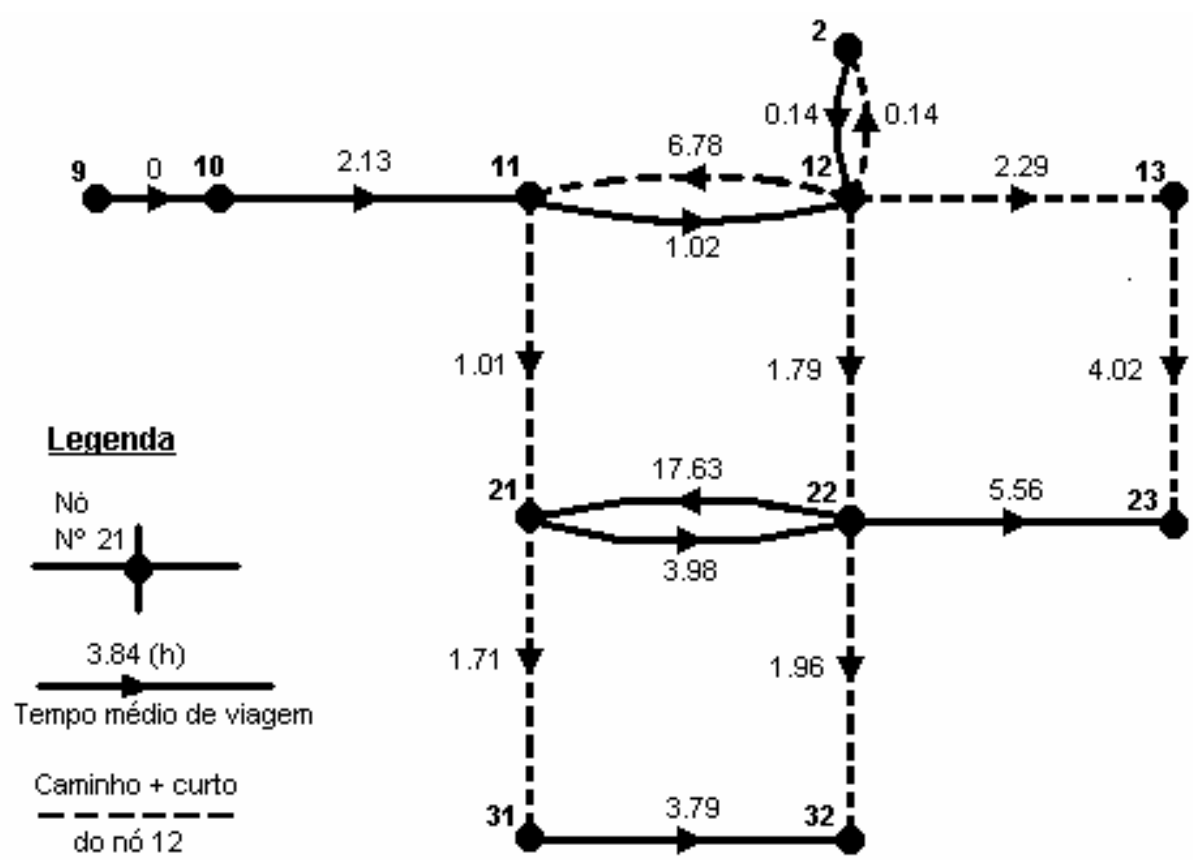

Figura 5.3 - Rede auxiliar para Tempo de viagem da Rede Net1

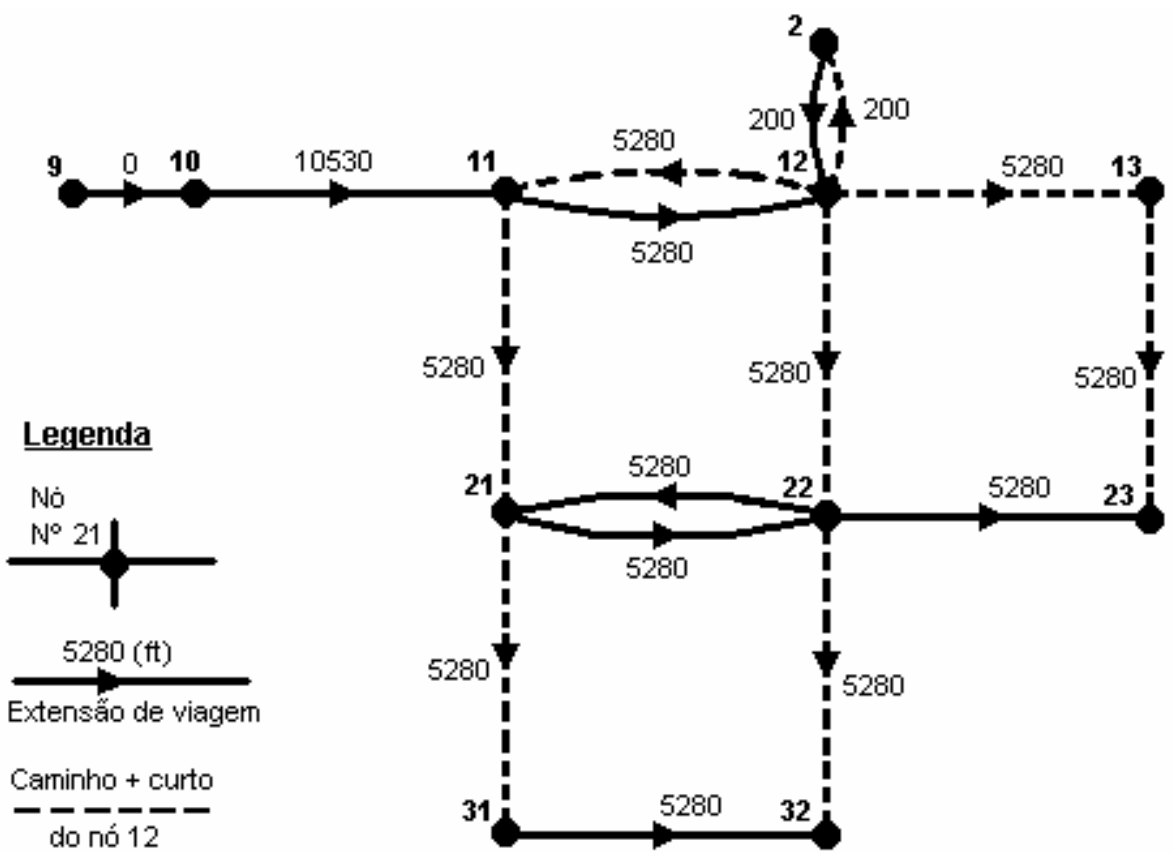

Figura 5.4 - Rede auxiliar para Extensão de viagem da Rede Net1 


\subsection{Determinação das Matrizes de Poluição}

As Matrizes de Poluição são construídas para representar os domínios de detecção e cobertura de cada nó da rede. É uma matriz N x N binária, sendo $\mathrm{N}$ é o número de nós, onde os elementos de valor "1" representam os nós contaminados e "0" os nós não contaminados. A linha $i$ indica todos os nós contaminados devido à intrusão no nó $i$. A coluna $j$ indica todos os nós poluição que podem contaminar o nó $j$.

Para a construção das Matrizes de Poluição foram identificados os objetivos a serem investigados

\subsubsection{Identificação dos objetivos}

Conforme mencionado anteriormente, a literatura apresenta cinco alternativas (possíveis objetivos) para o problema, detalhados a seguir.

\section{(1) População Exposta (PE):}

A População Exposta é a população com potencial para ser contaminada, ou seja, do momento do início da contaminação até sua detecção, quantas pessoas poderiam ser contaminadas. Deve-se minimizar a PE, porém para poder utilizar este objetivo deve-se conhecer, além da rede de distribuição de água, as regiões que esta abastece e vinculá-las à própria rede. Isto fica claro quando se compara um nó $x$ de demanda $d$ que representa uma indústria a um nó $y$ de demanda também $d$, que abastece porém uma escola ou um condomínio residencial. A população exposta no caso do nó $y$ é maior que a do nó $x$. 


\section{(2) Tempo para Detecção (TD):}

O Tempo para Detecção é o tempo entre a intrusão intencional e a detecção do contaminante pelos equipamentos de monitoramento. Deve-se minimizar TD, porém sua estimativa fica limitada ao tipo de contaminante, se este reage ou se comporta como um traçador.

\section{(3) Volume Consumido (VC):}

O Volume Consumido é a quantidade de água contaminada consumida pela população antes da detecção pelos sensores. A minimização de VC é considerada de maior relevância, uma vez que considera de modo direto o foco do trabalho, que é a segurança da população.

\section{(4) Extensão da Contaminação (EC):}

A Extensão da Contaminação, EC, é o comprimento total de tubulação contaminada pela intrusão, deve também ser minimizada.

\section{(5) Número de Detecções Falhas (NDF):}

O Número de Detecções Falhas, NDF, é a proporção de intrusão que são indetectáveis pelos sensores. Para considerar a minimização de NDF deve-se definir o contaminante e a probabilidade de detecção dos sensores, $a$ priori desconhecidos. Pode-se considerar a hipótese que o sensor detecta a proporção mínima que prejudique a saúde da população.

Dentre os objetivos citados, verifica-se que o Volume Consumido é o mais utilizado na literatura observada na revisão, com justificativa óbvia já 
descrita. Porém, tendo em vista que o principal alvo é a segurança da população (VC e TD) e economia (EC), a investigação de objetivos múltiplos faz-se necessária. Dentre os objetivos descritos, três serão investigados, sendo eles: o Volume Consumido, o Tempo de Detecção e a Extensão da Contaminação. Os outros objetivos listados acima não serão utilizados devido à necessidade de especificação de maiores detalhes da rede, da população (densidade), do contaminante (concentração) e do equipamento, a priori desconhecidos.

\subsubsection{Construção da Matriz Poluição para Volume Consumido}

A Matriz Poluição para Volume Consumido é estabelecida através dos seguintes passos:

- atribuir "0" inicialmente para todos os elementos da linha i da matriz;

- classificar todos os nós alcançáveis pela intrusão do nó i, da rede auxiliar, de acordo com o roteamento do caminho mais curto, em ordem crescente;

- iniciar a partir do primeiro nó classificado. Calcular o volume de água contaminada que é consumida antes da chegada da poluição vinda do nó $i$ ao nó classificado $j$. Se o volume exceder o máximo permitido (Nível de serviço 1: Volume Consumido) deve-se parar. Caso contrário, atribuir " 1 " ao elemento da linha $i$ e coluna $j$ da matriz e repetir o procedimento para o próximo nó classificado. Observa-se a seguir a Matriz Poluição para Volume Consumido de água contaminada para a rede Net1. 
Matriz Poluição para Volume Consumido (nível de serviço: 283.17m3):

\begin{tabular}{|c|cccccccccccc|}
\hline Nós: & 10 & 11 & 12 & 13 & 21 & 22 & 23 & 31 & 32 & 9 & 2 \\
\hline 10 & 0 & 1 & 1 & 0 & 1 & 1 & 0 & 1 & 0 & 0 & 1 \\
11 & 0 & 1 & 1 & 0 & 1 & 1 & 0 & 1 & 0 & 0 & 1 \\
12 & 0 & 0 & 1 & 1 & 0 & 1 & 0 & 0 & 1 & 0 & 1 \\
13 & 0 & 0 & 0 & 1 & 0 & 0 & 1 & 0 & 0 & 0 & 0 \\
21 & 0 & 0 & 0 & 0 & 1 & 1 & 0 & 1 & 0 & 0 & 0 \\
22 & 0 & 0 & 0 & 0 & 0 & 1 & 0 & 0 & 1 & 0 & 0 \\
23 & 0 & 0 & 0 & 0 & 0 & 0 & 1 & 0 & 0 & 0 & 0 \\
31 & 0 & 0 & 0 & 0 & 0 & 0 & 0 & 1 & 1 & 0 & 0 \\
32 & 0 & 0 & 0 & 0 & 0 & 0 & 0 & 0 & 1 & 0 & 0 \\
9 & 1 & 1 & 1 & 0 & 1 & 1 & 0 & 1 & 0 & 0 & 1 \\
2 & 0 & 0 & 1 & 1 & 0 & 1 & 0 & 0 & 1 & 0 & 0 \\
\hline
\end{tabular}

\subsubsection{Construção da Matriz Poluição para Tempo de Deteç̧ão}

A Matriz Poluição para Tempo de Detecção é estabelecida através dos seguintes passos:

- atribuir " 0 " inicialmente para todos os nós da linha $i$ da matriz;

- iniciar da primeira linha $i$ e coluna $\mathrm{j}$ até a última linha $i$ e coluna $j$. Avaliar cada elemento $c_{i j}$ da Matriz Custo para Tempo de viagem e o nível de serviço 2. Se o elemento $\mathrm{c}_{\mathrm{ij}}$ da Matriz Custo for menor que tempo máximo permitido (Nível de serviço 2: Tempo de Detecção) deve-se atribuir " 1 " ao elemento da linha $i$ e coluna $j$ da Matriz Custo para a Matriz Poluição e assim por diante, analogamente à matriz poluição para VC. Observa-se a seguir a Matriz Poluição para Tempo de Detecção para a rede Net1. 
Matriz Poluição para Tempo de Detecção (nível de serviço: 6.00h.)

\begin{tabular}{|c|ccccccccccc|}
\hline Nós: & 10 & 11 & 12 & 13 & 21 & 22 & 23 & 31 & 32 & 9 & 2 \\
\hline 10 & 1 & 1 & 1 & 1 & 1 & 1 & 0 & 1 & 0 & 0 & 1 \\
11 & 0 & 1 & 1 & 1 & 1 & 1 & 0 & 1 & 1 & 0 & 1 \\
12 & 0 & 0 & 1 & 1 & 0 & 1 & 0 & 0 & 1 & 0 & 1 \\
13 & 0 & 0 & 0 & 1 & 0 & 0 & 1 & 0 & 0 & 0 & 0 \\
21 & 0 & 0 & 0 & 0 & 1 & 1 & 0 & 1 & 1 & 0 & 0 \\
22 & 0 & 0 & 0 & 0 & 0 & 1 & 1 & 0 & 1 & 0 & 0 \\
23 & 0 & 0 & 0 & 0 & 0 & 0 & 1 & 0 & 0 & 0 & 0 \\
31 & 0 & 0 & 0 & 0 & 0 & 0 & 0 & 1 & 1 & 0 & 0 \\
32 & 0 & 0 & 0 & 0 & 0 & 0 & 0 & 0 & 1 & 0 & 0 \\
9 & 1 & 1 & 1 & 1 & 1 & 1 & 0 & 1 & 0 & 1 & 1 \\
2 & 0 & 0 & 1 & 1 & 0 & 1 & 0 & 0 & 1 & 0 & 1 \\
\hline
\end{tabular}

\subsubsection{Construção da Matriz Poluição para Extensão da}

\section{Contaminação}

A Matriz Poluição para Extensão da Contaminação é estabelecida através dos seguintes passos:

- atribuir " 0 " inicialmente para todos os nós da linha $i$ da matriz;

- iniciar da primeira linha $i$ e coluna $j$ até a última linha $i$ e coluna $j$. Avaliar cada elemento $\mathrm{c}_{\mathrm{ij}}$ da Matriz Custo para Extensão de viagem e o nível de serviço 3. Se o elemento $\mathrm{c}_{\mathrm{ij}}$ da Matriz Custo for menor que a extensão máxima permitida (Nível de serviço 3: Extensão da Contaminação) deve-se atribuir " 1 " da respectiva linha $i$ e a coluna $j$ da Matriz Custo para a Matriz Poluição e assim por diante, analogamente à matriz poluição para VC. Observa-se a seguir a Matriz Poluição para Extensão da Contaminação para a rede Net1. 
Matriz Poluição para Extensão da Contaminação (nível de serviço: 15.00\%):

\begin{tabular}{|c|ccccccccccc|}
\hline Nós: & 10 & 11 & 12 & 13 & 21 & 22 & 23 & 31 & 32 & 9 & 2 \\
\hline 10 & 1 & 0 & 0 & 0 & 0 & 0 & 0 & 0 & 0 & 0 & 0 \\
11 & 0 & 1 & 1 & 0 & 1 & 0 & 0 & 0 & 0 & 0 & 1 \\
12 & 0 & 1 & 1 & 1 & 0 & 1 & 0 & 0 & 0 & 0 & 1 \\
13 & 0 & 0 & 0 & 1 & 0 & 0 & 1 & 0 & 0 & 0 & 0 \\
21 & 0 & 0 & 0 & 0 & 1 & 1 & 0 & 1 & 0 & 0 & 0 \\
22 & 0 & 0 & 0 & 0 & 1 & 1 & 1 & 0 & 1 & 0 & 0 \\
23 & 0 & 0 & 0 & 0 & 0 & 0 & 1 & 0 & 0 & 0 & 0 \\
31 & 0 & 0 & 0 & 0 & 0 & 0 & 0 & 1 & 1 & 0 & 0 \\
32 & 0 & 0 & 0 & 0 & 0 & 0 & 0 & 0 & 1 & 0 & 0 \\
9 & 1 & 0 & 0 & 0 & 0 & 0 & 0 & 0 & 0 & 1 & 0 \\
2 & 0 & 1 & 1 & 1 & 0 & 1 & 0 & 0 & 0 & 0 & 1 \\
\hline
\end{tabular}

\subsection{Inicialização do Programa PLEDECORDA_MOM}

O programa denominado PLEDECORDA_MOM (Programa de Localização de Equipamentos para DEtecção de COntaminação em Rede de Distribuição de Água - Parte 2 - Otimização com Algoritmos Genéticos Multiobjetivo - SPEA) foi produzido no software Builder e com auxílio da biblioteca de domínio público MOMHLib++ (Jaszkiewicz, 1999) que tem implementado, dentre outros, o Algoritmo Genético Multiobjetivo SPEA, utilizado nas análises de otimização deste trabalho. O Programa importa de um arquivo .INP, o número de nós da rede e as matrizes poluição, além do número de equipamentos de monitoramento a adotar, além do indicativo relativo a considerar-se ou não o monitoramento por diversos equipamentos de detecção. Como resultado, o programa exporta o arquivo PLEDECORDA_MOM.TXT, com as Frentes de Pareto ou conjunto de soluções não-dominadas identificadas. Ver anexo III o arquivo PLEDECORDA_MOM.TXT para a Rede Net1. 


\subsection{Determinação dos Nós de Monitoramento}

\subsubsection{O Problema a ser tratado}

Um problema de otimização multiobjetivo considerando $p$ objetivos, $n$ variáveis de decisão e $m$ restrições gerais, geralmente pode ser expresso por:

$$
\begin{array}{lll}
\text { Maximizar } F=\left[f_{k}\left(x_{1}, x_{2}, \ldots, x_{n}\right),\right. & k=1,2, \ldots, p] \\
\text { sujeito à } & g_{i}\left(x_{1}, x_{2}, \ldots, x_{n}\right) & i=1,2, \ldots, m \\
& X_{j} \geq 0 & j=1,2, \ldots, n
\end{array}
$$

sendo $f_{k}$ as funções objetivo que compõe o conjunto multiobjetivo $F, g_{i}$ as restrições do problema e $\mathrm{X}=\left(\mathrm{x}_{1}, \mathrm{x}_{2}, \ldots, \mathrm{x}_{\mathrm{n}}\right)$ representa o vetor de $\mathrm{n}$ variáveis de decisão $x_{j}$.

Em um problema multiobjetivo, geralmente busca-se um conjunto de soluções consideradas melhores, pois não existe uma única solução ótima que satisfaça todos os objetivos da mesma forma. Este conjunto de soluções é denominado não-inferior, não-dominado, de compromisso ou Pareto ótimas. Tais soluções são superiores às demais soluções viáveis no espaço de busca.

\section{Conceito de Pareto ótimo:}

Maximizando os $\mathrm{n}$ componentes de um vetor função objetivo $\mathrm{F}$ e de um vetor variáveis de decisão X pertencente à em um universo U:

$$
F(X)=\left(f_{1}(X), \ldots, f p(X)\right)
$$


Um vetor decisão $X_{u} \in U$ é considerado Pareto ótimo se e somente se nenhum $X_{v} \in U$ para qual $v=F\left(X_{v}\right)=\left(v_{1}, v_{2}, \ldots, v_{p}\right)$ domina $u=F\left(X_{u}\right)=$ $\left(\mathrm{u}_{1}, \mathrm{u}_{2}, \ldots, \mathrm{u}_{\mathrm{p}}\right)$, isto é, não existe $\mathrm{X}_{\mathrm{v}} \in \mathrm{U}$ tal que $\forall i \in\{1, \ldots, p\}, v_{1} \leq u_{i} \wedge \exists i \in\{1, \ldots, p\} \mid v_{i}<u_{i}$. O conjunto de todos os vetores decisão é denominado Pareto ótimo, o conjunto de vetores objetivos correspondentes é denominado não dominado.

Para exemplificar o conceito acima, COHON (1978) apresenta um problema de maximização com 3 objetivos $\left(f_{1}, f_{2}\right.$ e $\left.f_{3}\right)$.

Tabela 5.3 - Maximização com 3 objetivos

\begin{tabular}{|c|c|c|c|c|}
\hline Alternativa & $\mathrm{f}_{1}$ & $\mathrm{f}_{2}$ & $\mathrm{f}_{3}$ & Solução \\
\hline \hline $\mathrm{A}$ & 5 & 8 & 7 & não inferior \\
\hline $\mathrm{B}$ & 4 & 9 & 3 & não inferior \\
\hline $\mathrm{C}$ & 4 & 4 & 4 & inferior \\
\hline $\mathrm{D}$ & 3 & 10 & 6 & não inferior \\
\hline $\mathrm{E}$ & 2 & 9 & 8 & não inferior \\
\hline
\end{tabular}

Ao comparar as alternativas A,B, C, D e E, segundo $f_{1}, f_{2}$ e $f_{3}$, permite-se concluir que A, B, D e E são não inferiores, enquanto C é inferior. Verifica-se que A tem melhores valores para $f_{1}$ e $f_{3}$ e pior valor de $f_{2}$ que $B$, portanto ambas são não inferiores, e A possui valores para $f_{1}, f_{2}$ e $f_{3}$ melhores que $C$, enquanto $B$ possui valores melhores para $f_{2}$ e $f_{3}$ que $C$, portanto $A$ domina C.

\subsubsection{O princípio dos Algoritmos Genéticos (AGs)}

Os algoritmos Genéticos são técnicas de busca estocásticas que imitam matematicamente os mecanismos de evolução natural, compreendendo os processos de seleção e genética das populações, em analogia como o 
processo biológico de sobrevivência e adaptação dos indivíduos mais capazes, devidamente tratadas em livros texto como GOLDBERG (1989). São atualmente enfocados na tentativa de dar tratamento a problemas de otimização complexos dos mais diversos. Os AGs diferem dos métodos de otimização clássicos, pois partem de um conjunto de soluções alternativas aleatórias denominada população, onde cada indivíduo (cromossomo) representa uma solução para o problema, que reúne um conjunto de atributos na forma de códigos. Tais atributos permitem que os indivíduos sejam avaliados e ordenados de acordo com o valor correspondente da Função de Aptidão, segundo o qual são reproduzidos ou não. A partir daí, uma nova população é criada. Cromossomos, denominados descendentes, são formados pela união de dois cromossomos pais da geração atual, através do operador recombinação (crossover), e, em seguida modificados através do operador mutação (mutation).

\subsubsection{Os AGs Multiobjetivo}

Os algoritmos genéticos são ferramentas naturais no tratamento multiobjetivo, por tratarem-se de métodos populacionais (DEB, 2001). Assim, as técnicas evolucionárias para otimização multiobjetivo mostram-se ferramentas adequadas para dar tratamento ao problema.

Dentre as técnicas evolucionárias de otimização multiobjetivo, destacam-se aquelas baseadas no conceito de soluções não dominadas de Pareto, por tratarem os problemas de maneira mais abrangente, considerando os diversos objetivos simultaneamente. A literatura indica uma grande diversidade 
de métodos evolucionários multiobjetivo, classificados em não-elitistas e elitistas, como os AGs Multiobjetivo de $2^{\mathrm{a}}$ geração (SPEA, NSGA, NSGA-II, MOMGA, PAES, entre outros).

ZIZTLER e THIELE (1998) introduziram o elitismo através da manutenção de uma população externa. Esta população armazena um número fixo de soluções não-dominadas encontradas no início da simulação. A cada geração, as novas soluções não-dominadas são comparadas à população externa existente e as soluções não-dominadas resultantes são preservadas. Este algoritmo é chamado Strength Pareto Evolutionary (SPEA), que além de preservar as melhores soluções, também as utiliza nas operações genéticas com as populações correntes, direcionando a população a se encaminhar em direção às boas regiões do espaço de busca. O trabalho de CHEUNG (2004) realizou um estudo comparativo de métodos de algoritmos genéticos multiobjetivo para análise de reabilitação de redes de distribuição de água para abastecimento. CHEUNG (2004) fez uma análise comparativa entre MultiObjective Genetic Algorithm (MOGA), Non-dominated Sorting Genetic Algorithm (NSGA), elitist Non-dominated Sorting Genetic Algorithm (NSGA-II) e o SPEA e concluiu que o algoritmo SPEA obteve melhor desempenho. Outro trabalho, CHEUNG et al. (2003), para tomada de decisão também para reabilitação de redes de distribuição de água para abastecimento, também fez um estudo comparativo de dois métodos, MOGA e SPEA, e concluiu que o algoritmo SPEA superou o algoritmo MOGA em relação às Frentes de Pareto produzidas e o tempo de processamento. 
Sendo assim, entre as versões mais atuais dos métodos evolucionários multiobjetivo, o algoritmo SPEA se destaca como um procedimento elitista de bom desempenho e será, portanto, utilizado no desenvolvimento deste trabalho. Para tanto, pretende-se utilizar a MOMHLib++, que está implementado o algoritmo SPEA, na linguagem $\mathrm{C}++$.

\subsubsection{SPEA}

No SPEA (Strength Pareto Evolutionary Algorithm), as melhores soluções (soluções não-dominadas) de cada iteração, denominadas elite, são armazenadas em um conjunto externo (temporário). Em cada iteração do algoritmo, as soluções externas são comparadas com as soluções pertencentes ao conjunto corrente de forma que as melhores soluções são preservadas.

Os dados de entrada para este algoritmo são:

- Dimensão da população (POP);

- Probabilidade de recombinação (Pr);

- Probabilidade de mutação (Pm);

- Dimensão da população externa (POPe);

- Número máximo de gerações (GER).

Os dados de entrada utilizados pelo SPEA no programa PLEDECORDA_MOM são:

- Dimensão da população (POP): 50;

- Probabilidade de recombinação (Pr): 80\%;

- Probabilidade de mutação (Pm): 10\%;

- Dimensão da população externa (POPe): 80; 
- Número máximo de gerações (GER): 20000;

- operador utilizado para recombinação: Blend Crossover (BLX- $\alpha$ );

- operador utilizado para mutação: Mutação Uniforme.

As etapas do procedimento podem ser descritas:

- Etapa 1: Geração da população inicial aleatória Pt considerando os parâmetros de entrada POP;

- Etapa 2: Criação da população externa Pte vazia de dimensão POPe;

- Etapa 3: Avaliação das funções objetivo;

- Etapa 4: Atualização da POPe:

o Identificação das soluções não dominadas da população Pt;

o Cópias das soluções não dominadas encontradas em Pt para Pte;

o Remoção das soluções não dominadas de Pt;

o Adequação do conjunto externo, conforme dimensão da população externa POPe, através de um algoritmo de cluster.

- Etapa 5: Nessa etapa o algoritmo verificará seu critério de convergência. Em geral, adota-se o número máximo de iterações para essa verificação. Se tal critério for satisfeito o algoritmo segue para a etapa 11 e o processo é finalizado, caso contrário, o algoritmo segue para a etapa 6 .

- Etapa 6: Avaliação SPEA

o O módulo de avaliação tem objetivo de atribuir valores de aptidão as soluções como forma de preparar para ao operador seleção. No SPEA, esse procedimento é realizado sob duas etapas. Primeiro todos vetores solução i pertencentes à população externa $(\mathrm{Pt})$ são classificados e recebem um valor de aptidão $S_{i}$ denominado na literatura internacional como strength. Esse valor de aptidão, para um dado vetor de solução 
i, é proporcional ao número de soluções dominadas pela solução $i$ na população corrente.

o Posteriormente, calculam-se os valores de aptidão ( $\mathrm{Fj})$ de todas as soluções $j$ pertencentes à população corrente (Pte). Esse valor de aptidão é calculado como sendo a soma de todos os valores de aptidão $S i$ das soluções externas $i$ que dominam vetores $j$ da população corrente.

- Etapa 7: Seleção:

o Nessa etapa o SPEA difere dos métodos MOGA e NSGA, pois, a técnica de seleção utilizada no SPEA é a seleção por torneio. Após todas as soluções, pertencentes aos conjuntos externo e corrente, terem recebido seus respectivos valores de aptidão (F), os conjuntos Pt e Pte são unidos para aplicação do operador de seleção.

o A seleção por torneio no SPEA é desenvolvida da seguinte forma: cria-se um conjunto temporário $\left(\mathrm{P}_{\text {temp }}\right)$ de dimensão $n$; seleciona-se aleatoriamente duas soluções $(i$ e $j$ ) pertencentes ao conjunto unificado (Pt U Pte ); se $(F j)<(F i)$ então $i$ é incluso em $\mathrm{P}_{\text {temp }}$ caso contrário $j$ é incluso em $\mathrm{P}_{\text {temp. }}$.

- Etapa 8: Recombinação: Nessa etapa o SPEA se assemelha aos AEs simples. A recombinação é denominada operador de variação. Esse operador é aplicado como na estrutura geral dos AGs convencionais simples.

- Etapa 9: Mutação: O operador de mutação também faz parte do processo de variação e é responsável pela introdução de diversidade na população, que muitas vezes é perdida ao longo das iterações, em outras palavras pode-se dizer que esse operador é responsável pela introdução de material genético na população.

- Etapa 10: Nova População: Uma nova população é formada devido à aplicação dos operadores de recombinação e mutação. Assim é necessário que uma nova avaliação seja realizada para cada vetor 
solução pertencente a essa nova população, portanto volta-se a etapa 3.

- Etapa 11: Nessa etapa o algoritmo apresenta as soluções ótimas Pareto (Pte).

\subsubsection{Implementação dos AGs multiobjetivo sobre as Matrizes de Poluição}

\subsubsection{Funções de Aptidão}

As funções de aptidão do problema foram avaliadas com base nas matrizes poluição de níveis de serviço em termos do volume máximo consumido de água contaminada (MP1), tempo máximo para detecção (MP2), extensão máxima da contaminação (MP3) e no número de pontos de monitoramento. Pretende-se, portanto, maximizar o número de fontes detectadas pelos nós solução dentro dos limites máximos para o volume consumido, o tempo de detecção e o comprimento da contaminação préestabelecidos para a definição das respectivas matrizes de poluição. As Funções F1, F2 e F3 a serem maximizadas foram expressas como:

Maximizar $\quad F 1=\sum_{j=1}^{n \text { var }} \sum_{i=1}^{\text {nnode }}\left(m p 1_{i, x j}\right)-$ DEC 1

em que :

DEC $1=$ DEC $2 * \sum_{j=1}^{n \text { var }}\left[\sum_{l i=1}^{\text {nnode }}\left(m p 1_{l i, x j}\right)-1\right]$

$\sum_{l i=1}^{\text {nnode }}\left(m p 1_{l i, x j}\right) \geq 1 \rightarrow$ DEC $2=0,99$

$\sum_{l i=1}^{\text {nnode }}\left(m p 1_{l i, x j}\right)=0 \rightarrow$ DEC $2=0$

$\left(m p 1_{l i, x j}, m p 1_{i, x j}\right) \in(0,1)$ 
Maximizar $\quad F 2=\sum_{j=1}^{n \text { var }} \sum_{i=1}^{\text {nnode }}\left(m p 2_{i, x j}\right)-$ DEC 1

em que :

DEC $1=D E C 2 * \sum_{j=1}^{n \operatorname{var}}\left[\sum_{l i=1}^{\text {nnode }}\left(m p 2_{l i, x j}\right)-1\right]$

$\sum_{l i=1}^{\text {nnode }}\left(m p 2_{l i, x j}\right) \geq 1 \rightarrow$ DEC $2=0,99$

$\sum_{l i=1}^{\text {nnode }}\left(m p 2_{l i, x j}\right)=0 \rightarrow$ DEC $2=0$

$\left(m p 2_{l i, x j}, m p 2_{i, x j}\right) \in(0,1)$

Maximizar $\quad F 3=\sum_{j=1}^{n} \sum_{i=1}^{n \text { var }}\left(m p 3_{i, x j}\right)-$ DEC 1

em que :

DEC $1=$ DEC $2 * \sum_{j=1}^{n \operatorname{var}}\left[\sum_{l i=1}^{\text {nnode }}\left(m p 3_{l i, x j}\right)-1\right]$

$\sum_{l i=1}^{\text {nnode }}\left(m p 3_{l i, x j}\right) \geq 1 \rightarrow$ DEC $2=0,99$

$\sum_{l i=1}^{\text {nnode }}\left(m p 3_{l i, x j}\right)=0 \rightarrow$ DEC $2=0$

$\left(m p 3_{l i, x j}, m p 3_{i, x j}\right) \in(0,1)$

Nas funções F1, F2 e F3 acima, nnode é o número de nós da rede, nvar é o número de variáveis de decisão (número de equipamentos de monitoramento), xj é uma variável de decisão do vetor decisão $X$, i e li são incrementos da linha da matriz poluição, $m p 1_{i, x j}$ e $m p 1_{l i, x j}$ são elementos da matriz $M P 1, m p 2_{i, x j}$ e $m p 2_{l i, x j}$ são elementos da matriz $M P 2, m p 3_{i, x j}$ e $m p 3_{l i, x j}$ são elementos da matriz MP3, DEC2 é o valor do decréscimo por nó já monitorado.

As funções F1, F2 e F3 acima explicitadas são compostas de 2 termos. O primeiro (soma) corresponde ao número de total de detecções, pelos 
diversos equipamentos de monitoramento. O segundo termo faz com que as detecções repetidas sejam subtraídas do cômputo geral e, em seu lugar, se tenha o valor $(0,99) \times \mathrm{n}^{\mathrm{o}}$ de nós que detectam poluição emitida por determinada fonte já detectada por outro equipamento. Essa é a maneira utilizada neste trabalho para considerar as detecções repetidas e assim reforçar a confiabilidade do sistema de monitoramento estabelecido, admitindo que os equipamentos possam falhar.

Dessa forma, por exemplo, se dois nós são capazes de detectar a poluição de certa fonte, o primeiro termo da função retornará o valor $1+1=2$ e o segundo $0,99 \times 1=0,99$, então $F$ assumirá o valor 2-0,99=1,01. Assim a fração inteira 1 do número 1,01 indica que a fonte de poluição foi detectada e a parte fracionária 0,01 representa que mais 1 equipamento foi capaz de detectar a poluição emitida por essa fonte.

Caso o critério confiabilidade seja excluído das análises, basta substituir o valor 0,99 das equações (14), (18) e (22) por 1,00.

\subsubsection{Representação das soluções}

Os AGs multiobjetivo serão aplicados utilizando as matrizes definindo-se um número máximo de pontos de monitoramento (nvar) fixo para estabelecer o comprimento da cadeia representativa das soluções (string). Essa cadeia conterá, portanto, os nós de monitoramento (números inteiros de 1 a n = número de nós da rede) relativos a cada solução do problema. Para o caso da 
Rede Net1, as duas tabelas abaixo apresentam soluções geradas pelo programa PLEDECORDA_MOM.

Tabela 5.4 - Resultados da Rede Net1 não considerando deteç̧ões repetidas.

\begin{tabular}{|c|c|c|c|c|}
\hline \multicolumn{5}{|c|}{$\mathrm{N}^{\mathrm{o}}$ de equipamentos para monitoramento: 01un. } \\
\hline Solução & \multicolumn{3}{|c|}{ no espaço de objetivos } & no espaço de decisão \\
\hline $\mathrm{N}^{\mathrm{o}}$. & F1 & $\mathrm{F} 2$ & $\mathrm{~F} 3$ & Nó de monitoramento \\
\hline 1 & 7 & 7 & 4 & 22 \\
\hline \multicolumn{5}{|c|}{$\mathrm{N}^{0}$ de equipamentos para monitoramento: $02 \mathrm{un}$. } \\
\hline Solução & \multicolumn{3}{|c|}{ no espaço de objetivos } & no espaço de decisão \\
\hline $\mathrm{N}^{\mathrm{o}}$. & F1 & $\mathrm{F} 2$ & $\mathrm{~F} 3$ & Nós de monitoramento \\
\hline 1 & 9 & 9 & 6 & 23 e 22 \\
\hline 2 & 6 & 10 & 6 & 13 e 32 \\
\hline \multicolumn{5}{|c|}{$\mathrm{N}^{0}$ de equipamentos para monitoramento: 03un. } \\
\hline Solução & \multicolumn{3}{|c|}{ no espaço de objetivos } & no espaço de decisão \\
\hline $\mathrm{N}^{\mathrm{o}}$. & F1 & $\mathrm{F} 2$ & F3 & Nós de monitoramento \\
\hline 1 & 11 & 11 & 8 & 23,32 e 22 \\
\hline
\end{tabular}

Tabela 5.5 - Resultados da Rede Net1 considerando deteç̧ões repetidas $\mathrm{N}^{\mathrm{o}}$ de equipamentos para monitoramento: 01un.

\begin{tabular}{|c|c|c|c|c|}
\hline \hline Solução & \multicolumn{3}{|c|}{ no espaço de objetivos } & no espaço de decisão \\
\hline $\mathrm{N}^{\mathrm{o}}$. & $\mathrm{F} 1$ & $\mathrm{~F} 2$ & $\mathrm{~F} 3$ & Nós de monitoramento \\
\hline 1 & 7 & 7 & 4 & 22 \\
\hline \hline
\end{tabular}

$\mathrm{N}^{\mathrm{o}}$ de equipamentos para monitoramento: $02 \mathrm{un}$.

\begin{tabular}{|c|c|c|c|c|}
\hline \hline Solução & \multicolumn{3}{|c|}{ no espaço de objetivos } & no espaço de decisão \\
\hline $\mathrm{N}^{\mathrm{o}}$. & $\mathrm{F} 1$ & $\mathrm{~F} 2$ & $\mathrm{~F} 3$ & Nós de monitoramento \\
\hline 1 & 6.02 & 10.03 & 6 & 13 e 32 \\
\hline 2 & 9.03 & 9.05 & 6.01 & 32 e 22 \\
\hline \hline
\end{tabular}

$\mathrm{N}^{\mathrm{o}}$ de equipamentos para monitoramento: $03 \mathrm{un}$.

\begin{tabular}{||c|c|c|c|c||}
\hline \hline Solução & \multicolumn{3}{|c|}{ no espaço de objetivos } & no espaço de decisão \\
\hline $\mathrm{N}^{\mathrm{o}}$. & $\mathrm{F} 1$ & $\mathrm{~F} 2$ & $\mathrm{~F} 3$ & Nós de monitoramento \\
\hline 1 & 11.03 & 11.06 & 8.02 & 22,23 e 32 \\
\hline
\end{tabular}




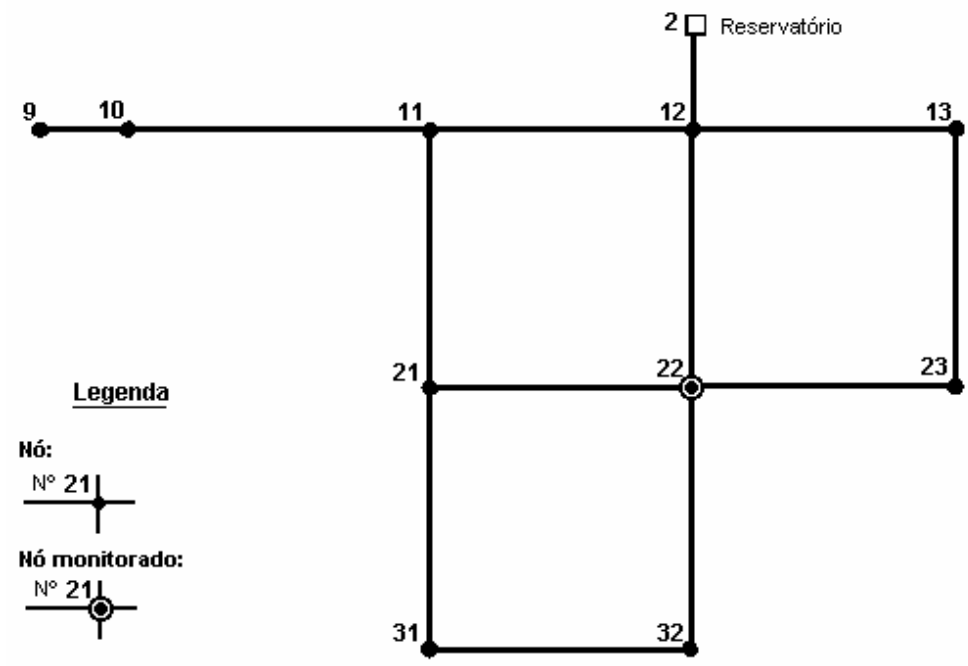

Figura 5.5 - Solução 1 da Rede Net1 para 1 equipamento considerando detecções repetidas.

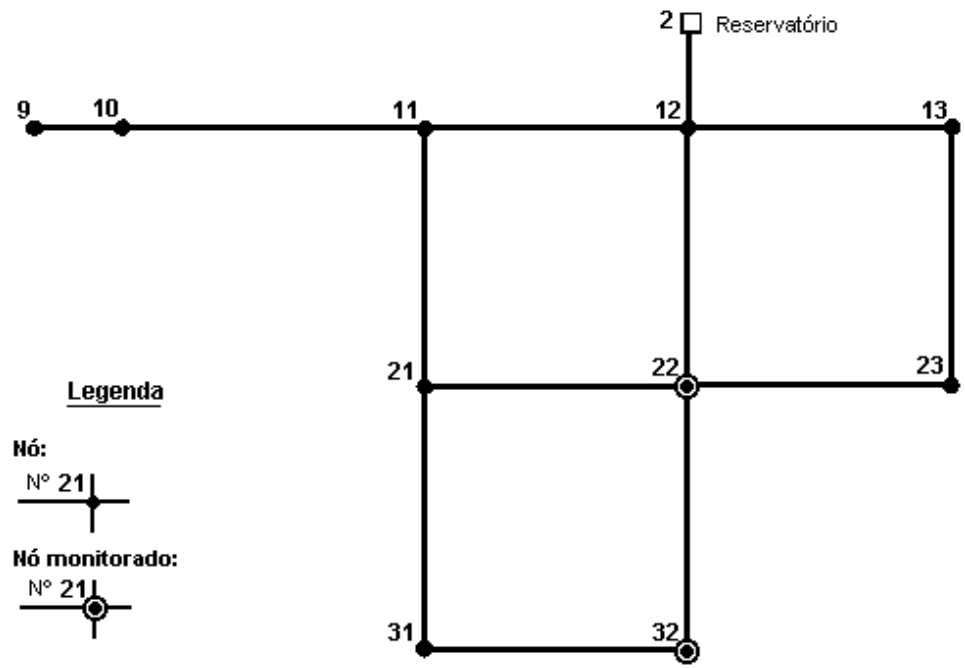

Figura 5.6 - Solução 1 da Rede Net1 para 2 equipamento considerando detecções repetidas. 


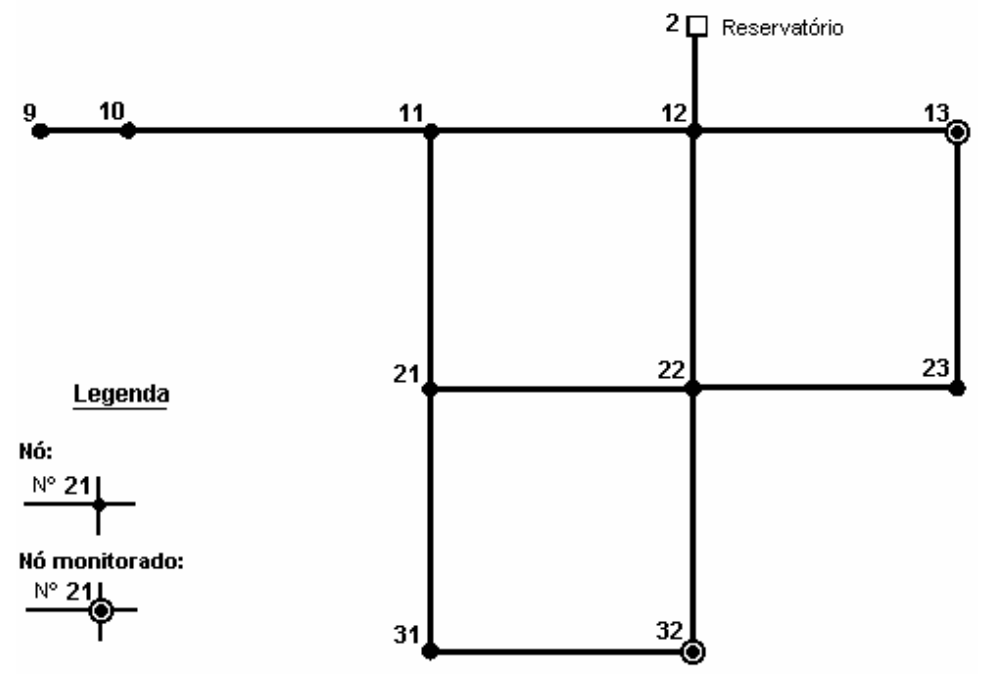

Figura 5.7 - Solução 2 da Rede Net1 para 2 equipamento considerando detecções repetidas.

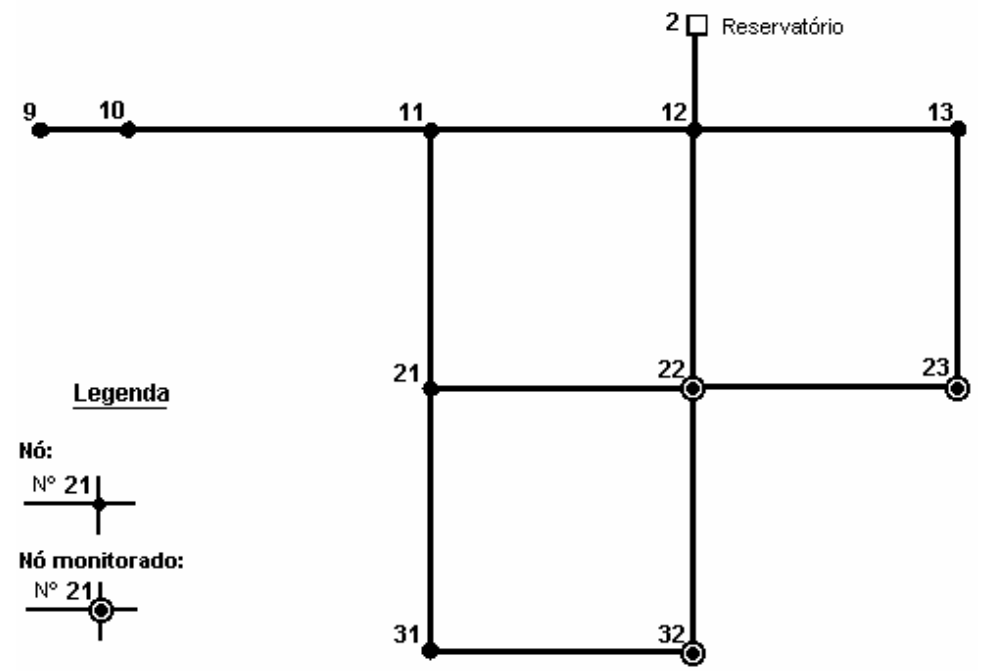

Figura 5.8 - Solução 1 da Rede Net1 para 3 equipamento considerando detecções repetidas.

Para melhor entendimento, examina-se a solução 1 da tabela 5.5 com três equipamentos para monitoramento (nvar=3) e com acréscimo de nó já monitorado, correspondente ao vetor $(22,23$ e 32$)$. A partir das matrizes de poluição já determinadas, podem-se avaliar as três funções de aptidão do problema no ponto representado pela referida solução, da seguinte forma: 
Matriz Poluição para Volume Consumido (nível de serviço: $283.17 \mathrm{~m} 3$ ):

\begin{tabular}{|c|c|c|c|c|c|c|c|c|c|c|c|c|c|}
\hline \multirow{2}{*}{\multicolumn{2}{|c|}{ Nós: }} & \multicolumn{11}{|c|}{ Nós poluídos/contaminados } & \multirow{2}{*}{$\begin{array}{c}\text { Detecção } \\
\text { repetida }\end{array}$} \\
\hline & & 10 & 11 & 12 & 13 & 21 & 22 & 23 & 31 & 32 & 10 & 11 & \\
\hline \multirow{11}{*}{ 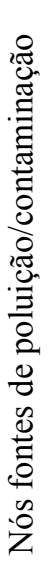 } & 10 & 0 & 1 & 1 & 0 & 1 & 1 & 0 & 1 & 0 & 0 & 1 & \\
\hline & 11 & 0 & 1 & 1 & 0 & 1 & 1 & 0 & 1 & 0 & 0 & 1 & \\
\hline & 12 & 0 & 0 & 1 & 1 & 0 & 1 & 0 & 0 & 1 & 0 & 1 & 1 \\
\hline & 13 & 0 & 0 & 0 & 1 & 0 & 0 & 1 & 0 & 0 & 0 & 0 & \\
\hline & 21 & 0 & 0 & 0 & 0 & 1 & 1 & 0 & 1 & 0 & 0 & 0 & \\
\hline & 22 & 0 & 0 & 0 & 0 & 0 & 1 & 0 & 0 & 1 & 0 & 0 & 1 \\
\hline & 23 & 0 & 0 & 0 & 0 & 0 & 0 & 1 & 0 & 0 & 0 & 0 & \\
\hline & 31 & 0 & 0 & 0 & 0 & 0 & 0 & 0 & 1 & 1 & 0 & 0 & \\
\hline & 32 & 0 & 0 & 0 & 0 & 0 & 0 & 0 & 0 & 1 & 0 & 0 & \\
\hline & 9 & 1 & 1 & 1 & 0 & 1 & 1 & 0 & 1 & 0 & 0 & 1 & \\
\hline & 2 & 0 & 0 & 1 & 1 & 0 & 1 & 0 & 0 & 1 & 0 & 0 & 1 \\
\hline \multicolumn{2}{|c|}{ Soma } & & & & & & 7 & 2 & & 5 & & & 3 \\
\hline
\end{tabular}

Fontes detectadas pelo nó 22: $(10,11,12,21,22,9,2)$.

Fontes detectadas pelo nó 23: $(13,23)$.

Fontes detectadas pelo nó 32: $(12,22,31,32,2)$.

$\mathrm{F} 1=\mathrm{n}^{\mathrm{o}}$. de fontes detectadas pela solução $(22,23,32): 14[=7+2+5]-$ 3. $(0,99)=11,03$. Observa-se que o valor 11 significa o número total de nós fonte que são detectados e o número de nós da rede é 11, portanto todos os nós são monitorados dentro do especificado nível de serviço. $\mathrm{O}$ acréscimo 0,03 significa que há 3 repetições de nós já monitorados (nós 12, 22 e 2), como observado nas linhas da matriz. 
Matriz Poluição para Tempo de Detecção (nível de serviço: 6.00h.)

\begin{tabular}{|c|c|c|c|c|c|c|c|c|c|c|c|c|c|}
\hline \multirow{2}{*}{\multicolumn{2}{|c|}{ Nós: }} & \multicolumn{11}{|c|}{ Nós poluídos/contaminados } & \multirow{2}{*}{$\begin{array}{l}\text { Detecção } \\
\text { repetida }\end{array}$} \\
\hline & & 10 & 11 & 12 & 13 & 21 & 22 & 23 & 31 & 32 & 10 & 11 & \\
\hline \multirow{11}{*}{ 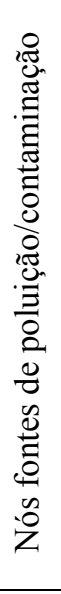 } & 10 & 1 & 1 & 1 & 1 & 1 & 1 & 0 & 1 & 0 & 0 & 1 & \\
\hline & 11 & 0 & 1 & 1 & 1 & 1 & 1 & 0 & 1 & 1 & 0 & 1 & 1 \\
\hline & 12 & 0 & 0 & 1 & 1 & 0 & 1 & 0 & 0 & 1 & 0 & 1 & 1 \\
\hline & 13 & 0 & 0 & 0 & 1 & 0 & 0 & 1 & 0 & 0 & 0 & 0 & \\
\hline & 21 & 0 & 0 & 0 & 0 & 1 & 1 & 0 & 1 & 1 & 0 & 0 & 1 \\
\hline & 22 & 0 & 0 & 0 & 0 & 0 & 1 & 1 & 0 & 1 & 0 & 0 & 2 \\
\hline & 23 & 0 & 0 & 0 & 0 & 0 & 0 & 1 & 0 & 0 & 0 & 0 & \\
\hline & 31 & 0 & 0 & 0 & 0 & 0 & 0 & 0 & 1 & 1 & 0 & 0 & \\
\hline & 32 & 0 & 0 & 0 & 0 & 0 & 0 & 0 & 0 & 1 & 0 & 0 & \\
\hline & 9 & 1 & 1 & 1 & 1 & 1 & 1 & 0 & 1 & 0 & 1 & 1 & \\
\hline & 2 & 0 & 0 & 1 & 1 & 0 & 1 & 0 & 0 & 1 & 0 & 1 & 1 \\
\hline \multicolumn{2}{|c|}{ Soma } & & & & & & 7 & 3 & & 7 & & & 6 \\
\hline
\end{tabular}

Fontes detectadas pelo nó 22: $(10,11,21,22,9,2)$.

Fontes detectadas pelo nó 23: $(13,22,23)$.

Fontes detectadas pelo nó 32: $(11,12,21,22,31,32,2)$.

$\mathrm{F} 2=\mathrm{n}^{\mathrm{o}}$. de fontes detectadas pela solução $(22,23,32): 17[=7+3+7]-$ $6 .(0,99)=11,06$. Observa-se que o valor 11 significa o número total de nós fonte que são detectados e o número de nós da rede é 11, portanto todos os nós são monitorados dentro do especificado nível de serviço. $O$ acréscimo 0,06 significa que há 6 repetições de nós já monitorados (nós 11, 12, 21, 22(2x) e 2), como observado nas linhas da matriz. 
Matriz Poluição para Extensão da Contaminação (nível de serviço: 15.00\%):

\begin{tabular}{|c|c|c|c|c|c|c|c|c|c|c|c|c|c|}
\hline \multirow{2}{*}{\multicolumn{2}{|c|}{ Nós: }} & \multicolumn{11}{|c|}{ Nós poluídos/contaminados } & \multirow{2}{*}{$\begin{array}{c}\text { Detecção } \\
\text { repetida }\end{array}$} \\
\hline & & 10 & 11 & 12 & 13 & 21 & 22 & 23 & 31 & 32 & 10 & 11 & \\
\hline \multirow{11}{*}{ 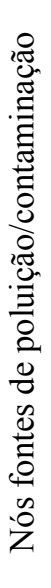 } & 10 & 1 & 0 & 0 & 0 & 0 & 0 & 0 & 0 & 0 & 0 & 0 & \\
\hline & 11 & 0 & 1 & 1 & 0 & 1 & 0 & 0 & 0 & 0 & 0 & 1 & \\
\hline & 12 & 0 & 1 & 1 & 1 & 0 & 1 & 0 & 0 & 0 & 0 & 1 & \\
\hline & 13 & 0 & 0 & 0 & 1 & 0 & 0 & 1 & 0 & 0 & 0 & 0 & \\
\hline & 21 & 0 & 0 & 0 & 0 & 1 & 1 & 0 & 1 & 0 & 0 & 0 & \\
\hline & 22 & 0 & 0 & 0 & 0 & 1 & 1 & 1 & 0 & 1 & 0 & 0 & 2 \\
\hline & 23 & 0 & 0 & 0 & 0 & 0 & 0 & 1 & 0 & 0 & 0 & 0 & \\
\hline & 31 & 0 & 0 & 0 & 0 & 0 & 0 & 0 & 1 & 1 & 0 & 0 & \\
\hline & 32 & 0 & 0 & 0 & 0 & 0 & 0 & 0 & 0 & 1 & 0 & 0 & \\
\hline & 9 & 1 & 0 & 0 & 0 & 0 & 0 & 0 & 0 & 0 & 1 & 0 & \\
\hline & 2 & 0 & 1 & 1 & 1 & 0 & 1 & 0 & 0 & 0 & 0 & 1 & \\
\hline \multicolumn{2}{|c|}{ Soma } & & & & & & 4 & 3 & & 3 & & & 2 \\
\hline
\end{tabular}

Fontes detectadas pelo nó 22: $(12,21,22,2)$.

Fontes detectadas pelo nó 23: $(13,22,23)$.

Fontes detectadas pelo nó 32: $(22,31,32)$.

$\mathrm{F} 3=\mathrm{n}^{\mathrm{o}}$. de fontes detectadas pela solução $(22,23,32): 10[=4+3+3]-$ 2. $(0,99)=\mathbf{8 , 0 2}$. Observa-se que o valor 8 significa o número total de nós fonte que são detectados e o número de nós da rede é 11, portanto nem todos os nós são monitorados dentro do especificado nível de serviço, deve-se então aumentar o nível de serviço para extensão da contaminação ou aumentar o número de equipamentos de monitoramento. $\mathrm{O}$ acréscimo 0,02 significa que há 2 repetições de nós já monitorados (nó $22(2 x)$ ), como observado nas linhas da matriz. 


\section{ESTUDO DE CASO: Rede Anytown}

\subsection{Simulação Hidráulica}

Para o segundo estudo de caso realizado, utilizou-se a Rede Anytown (WALSKI et al. 1987), utilizada também em outros trabalhos, como o de KESSLER et al. (1998), cuja representação esquemática é apresentada na figura 6.1. O sistema de distribuição é formado por 34 tubos, 19 nós, sendo destes nós, 2 tanques (reservatórios de nível variado) e 1 poço, o nível do poço é constante em $10 \mathrm{ft}$. As características dos tubos, dos nós e da bomba são mostradas nas tabelas a seguir.

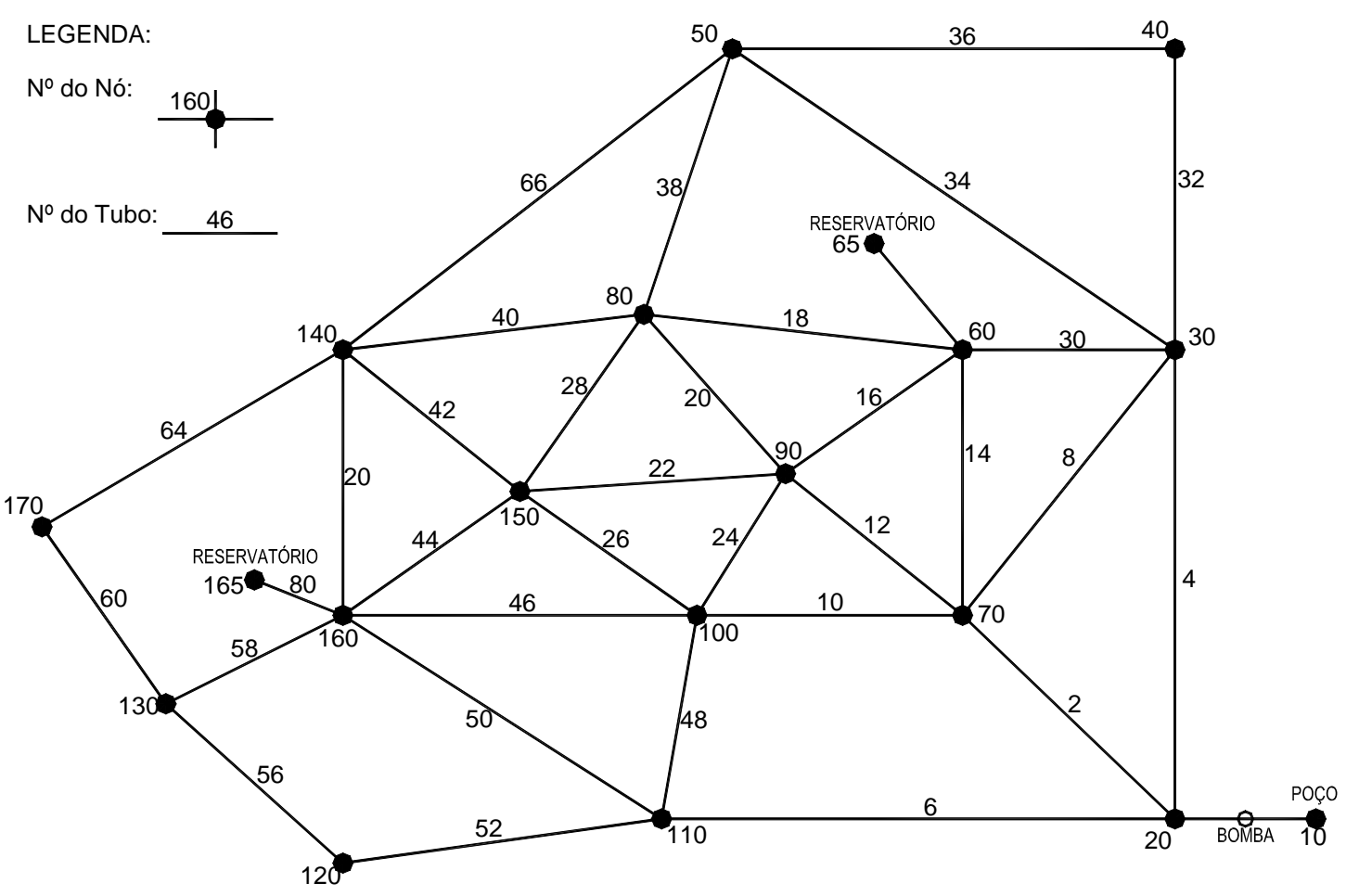

Figura 6.1 - Rede esquemática da Anytown 
Estudo da Localização Otimizada de Equipamentos em Rede

de Distribuição de Água para Detecção de Contaminação

Tabela 6.1 - Características dos tubos da Rede Anytown

\begin{tabular}{|c|c|c|c|}
\hline $\begin{array}{l}\text { Número } \\
\text { do tubo }\end{array}$ & $\begin{array}{c}\text { Comprimento } \\
\text { (ft) }\end{array}$ & $\begin{array}{l}\text { Diâmetro } \\
\text { (in.) }\end{array}$ & $\begin{array}{c}\text { Coeficiente de } \\
\text { rugosidade - C } \\
\text { Hazen-Willians }\end{array}$ \\
\hline 2 & 12000 & 16 & 120 \\
\hline 4 & 12000 & 12 & 120 \\
\hline 6 & 12000 & 12 & 120 \\
\hline 8 & 9000 & 12 & 120 \\
\hline 10 & 6000 & 12 & 120 \\
\hline 12 & 6000 & 10 & 120 \\
\hline 14 & 6000 & 12 & 120 \\
\hline 16 & 6000 & 10 & 120 \\
\hline 18 & 6000 & 12 & 120 \\
\hline 20 & 6000 & 10 & 120 \\
\hline 22 & 6000 & 10 & 120 \\
\hline 24 & 6000 & 10 & 120 \\
\hline 26 & 6000 & 12 & 120 \\
\hline 28 & 6000 & 10 & 120 \\
\hline 30 & 6000 & 10 & 120 \\
\hline 32 & 6000 & 10 & 120 \\
\hline 34 & 9000 & 10 & 120 \\
\hline 36 & 6000 & 10 & 120 \\
\hline 38 & 6000 & 10 & 120 \\
\hline 40 & 6000 & 10 & 120 \\
\hline 42 & 6000 & 8 & 120 \\
\hline 44 & 6000 & 8 & 120 \\
\hline 46 & 6000 & 8 & 120 \\
\hline 48 & 6000 & 8 & 120 \\
\hline 50 & 6000 & 10 & 120 \\
\hline 52 & 6000 & 8 & 120 \\
\hline 56 & 6000 & 8 & 120 \\
\hline 58 & 6000 & 10 & 120 \\
\hline 60 & 6000 & 8 & 120 \\
\hline 62 & 6000 & 8 & 120 \\
\hline 64 & 12000 & 8 & 120 \\
\hline 66 & 12000 & 8 & 120 \\
\hline 78 & 100 & 12 & 120 \\
\hline 80 & 100 & 12 & 120 \\
\hline
\end{tabular}


Tabela 6.2 - Características dos nós da Rede Anytown

\begin{tabular}{|c|c|c||}
\hline $\begin{array}{c}\text { Número } \\
\text { do nó }\end{array}$ & $\begin{array}{c}\text { Cota } \\
\text { (ft) }\end{array}$ & $\begin{array}{c}\text { Demanda média } \\
\text { (gal./min) }\end{array}$ \\
\hline \hline 20 & 20 & 500 \\
\hline 30 & 50 & 200 \\
\hline 40 & 50 & 200 \\
\hline 50 & 50 & 200 \\
\hline 60 & 50 & 500 \\
\hline 70 & 50 & 500 \\
\hline 80 & 50 & 500 \\
\hline 90 & 50 & 1000 \\
\hline 100 & 50 & 500 \\
\hline 110 & 50 & 500 \\
\hline 120 & 120 & 200 \\
\hline 130 & 120 & 200 \\
\hline 140 & 80 & 200 \\
\hline 150 & 120 & 200 \\
\hline 160 & 120 & 800 \\
\hline 170 & 120 & 200 \\
\hline
\end{tabular}

Tabela 6.3 - Características dos Tanques da Rede Anytown

\begin{tabular}{|c|c|c|c|c|c||}
\hline $\begin{array}{c}\text { Número do } \\
\text { Tanque } \\
\text { (Reservatório) }\end{array}$ & $\begin{array}{c}\text { Cota } \\
\text { (ft) }\end{array}$ & $\begin{array}{c}\text { Nível } \\
\text { Inicial } \\
\text { (ft) }\end{array}$ & $\begin{array}{c}\text { Nível } \\
\text { Mínimo } \\
\text { (ft) }\end{array}$ & $\begin{array}{c}\text { Nível } \\
\text { Máximo } \\
\text { (ft) }\end{array}$ & $\begin{array}{c}\text { Diâmetro } \\
\text { (ft) }\end{array}$ \\
\hline \hline 65 & 215 & 35 & 10 & 35 & 53.8 \\
\hline 165 & 215 & 35 & 10 & 35 & 53.8 \\
\hline
\end{tabular}

Tabela 6.4 - Características da bomba da Rede Anytown

\begin{tabular}{|c|c|}
\hline $\begin{array}{c}\text { Vazão } \\
\text { (gal./min) }\end{array}$ & $\begin{array}{c}\text { Altura Manométrica } \\
\text { (ft) }\end{array}$ \\
\hline 0 & 358 \\
\hline 12000 & 270 \\
\hline 24000 & 190 \\
\hline 33000 & 0 \\
\hline
\end{tabular}

Tabela 6.5 - Padrão diário de demanda para a Rede Anytown

\begin{tabular}{|c|c|}
\hline Hora do dia & Multiplicador da demanda média \\
\hline \hline $06-09$ & 1.2 \\
\hline $09-12$ & 1.3 \\
\hline $12-15$ & 1.2 \\
\hline $15-18$ & 1.1 \\
\hline $18-21$ & 1.0 \\
\hline $21-24$ & 0.9 \\
\hline $24-03$ & 0.7 \\
\hline $03-06$ & 0.6 \\
\hline
\end{tabular}




\subsection{Inicialização do PLEDECORDA_EPA}

$\mathrm{O}$ arquivo REDE.INP da Rede Anytown para o programa pode ser visto no Anexo IV e o arquivo de saída, PLEDECORDA_EPA.TXT, para a Rede Anytown, nos Anexos V e VII.

\subsubsection{Parâmetros de Entrada - Níveis de Serviço}

(1) Volume Consumido (VC): adotaram-se 02 valores para $\mathrm{VC}=468,10 \mathrm{~m}^{3}\left(16,530 \mathrm{ft}^{3}\right)$ e $1.245,15 \mathrm{~m}^{3}\left(43,972 \mathrm{ft}^{3}\right) ;$

(2) Tempo de Deteç̧ão (TD): adotou-se TD = 6 horas;

(3) Extensão da Contaminação (EC): adotou-se EC $=15 \%$.

\subsection{Construção das Redes Auxiliares e dos Caminhos Mais Curtos}

A aplicação do Algoritmo de Floyd permitiu que fossem identificadas as matrizes de Custo e Roteamento mostradas a seguir, que auxiliaram na construção das redes auxiliares. 
Estudo da Localização Otimizada de Equipamentos em Rede de Distribuição de Água para Detecção de Contaminação

Matriz Custo de Tempo de viagem Final (h) (Caminhos Mais Curtos):

\begin{tabular}{|c|c|c|c|c|c|c|c|c|c|c|c|c|c|c|c|c|c|c|c|}
\hline Nós & 20 & 30 & 40 & 50 & 60 & 70 & 80 & 90 & 100 & 110 & 120 & 130 & 140 & 150 & 160 & 170 & 10 & 65 & 165 \\
\hline 20 & 0 & .84 & .78 & .35 & 1.43 & 0.70 & 2.64 & .37 & 1.33 & 0.78 & 1.66 & 3.81 & 4.73 & 3.32 & 1.57 & 7.59 & 888 & 1.47 & 1.60 \\
\hline 30 & 88 & 0 & .94 & 1.52 & 0.83 & 888 & 2.05 & 2.22 & 84.14 & 888 & 888 & 8.64 & 4.14 & 7.23 & 6.40 & 7.91 & 888 & 0.87 & 6.43 \\
\hline 40 & 888 & 888 & 0 & 1.75 & 888 & 888 & 3.13 & 17.71 & 85.22 & 888 & 888 & 9.71 & 5.22 & 8.31 & 7.48 & 8.99 & 888 & 888 & 7.50 \\
\hline 50 & 888 & 888 & 888 & 0 & 888 & 888 & 1.38 & 15.95 & 83.46 & 888 & 888 & 7.96 & 3.46 & 6.55 & 5.72 & 7.23 & 888 & 888 & 5.75 \\
\hline 60 & 888 & 888 & 888 & 888 & 0 & 888 & 1.22 & 1.38 & 83.30 & 888 & 888 & 7.80 & 3.30 & 6.39 & 5.56 & 7.07 & 888 & 0.04 & 5.59 \\
\hline 70 & 888 & 7.28 & 8.22 & 8.79 & 0.73 & 0 & 1.94 & 0.67 & 0.63 & 888 & 888 & 4.25 & 4.03 & 2.62 & 2.01 & 7.80 & 888 & 0.76 & 2.04 \\
\hline 80 & 888 & 888 & 888 & 888 & 888 & 888 & 0 & 14.57 & 82.09 & 888 & 888 & 6.58 & 2.09 & 5.17 & 4.35 & 5.86 & 888 & 888 & 4.37 \\
\hline 90 & 888 & 888 & 888 & 888 & 888 & 888 & 20.75 & 0 & 85.16 & 888 & 888 & 9.66 & 8.37 & 5.67 & 7.42 & 12.14 & 888 & 888 & 7.45 \\
\hline 100 & 888 & 888 & 888 & 888 & 888 & 888 & 23.39 & 2.65 & 0 & 888 & 888 & 3.62 & 4.69 & 1.99 & 1.39 & 7.41 & 888 & 888 & 1.41 \\
\hline 110 & 888 & 888 & 888 & 888 & 888 & 888 & 24.76 & 4.02 & 1.37 & 0 & 0.88 & 3.02 & 6.06 & 3.37 & 0.79 & 6.81 & 888 & 888 & 0.82 \\
\hline 120 & 888 & 888 & 888 & 888 & 888 & 888 & 122.0 & 101.3 & 103.3 & 888 & 0 & 2.71 & 37.78 & 49.49 & 25.54 & 6.49 & 888 & 888 & 25.57 \\
\hline 130 & 888 & 888 & 888 & 888 & 888 & 888 & 119.33 & 98.59 & 100.58 & & 888 & 0 & 35.07 & 46.79 & 22.84 & 3.78 & 888 & 888 & 22.86 \\
\hline 140 & 888 & 888 & 888 & 888 & 888 & 888 & 98.76 & 78.01 & 80 & 888 & 888 & 4.50 & 0 & 26.21 & 2.26 & 3.77 & 888 & 888 & 2.29 \\
\hline 150 & 888 & 888 & 888 & 888 & 888 & 888 & 72.55 & 51.80 & 79.49 & 888 & 888 & 3.99 & 2.69 & 0 & 1.75 & 6.46 & 888 & 888 & 1.78 \\
\hline 160 & 888 & 888 & 888 & 888 & 888 & 888 & 96.50 & 75.75 & 77.74 & 888 & 888 & 2.24 & 12.24 & 23.95 & 0 & 6.02 & 888 & 888 & 0.03 \\
\hline 170 & 888 & 888 & 888 & 888 & 888 & 888 & 152.9 & 132.2 & 134.1 & 888 & 888 & 33.57 & 68.64 & 80.36 & 56.41 & 0 & 888 & 888 & 56.43 \\
\hline 10 & 0 & 0.84 & 1.78 & 2.35 & 1.43 & 0.70 & 2.64 & 1.37 & 1.33 & 0.78 & 1.66 & 3.81 & 4.73 & 3.32 & 1.57 & 7.59 & 0 & 1.47 & 1.60 \\
\hline 65 & 888 & 888 & 888 & 888 & 0.04 & 888 & 1.26 & 1.42 & 83.35 & 888 & 888 & 7.84 & 3.34 & 6.43 & 5.61 & 7.11 & 888 & 0 & 5.63 \\
\hline 165 & 888 & 888 & 888 & 888 & 888 & 888 & 96.52 & 75.77 & 77.76 & 888 & 888 & 2.26 & 12.26 & 23.97 & 0.02 & 6.04 & 888 & 888 & 0 \\
\hline
\end{tabular}

Matriz Custo de Extensão de viagem Final (ft) (Caminhos Mais Curtos):

\begin{tabular}{|c|c|c|c|c|c|c|c|c|c|c|c|c|c|c|c|c|c|c|c|}
\hline Nós & 20 & 30 & 40 & 50 & 60 & 70 & 80 & 90 & 100 & 110 & 120 & 130 & 140 & 150 & 160 & 170 & 10 & 65 & 165 \\
\hline 20 & 0 & 12000 & 18000 & 21000 & 18000 & 12000 & 24000 & 18000 & 18000 & 12000 & 18000 & 24000 & 24000 & 24000 & 18000 & 30000 & 228200 & 18100 & 18100 \\
\hline 30 & 228200 & 0 & 6000 & 9000 & 6000 & 228200 & 12000 & 12000 & 18000 & 228200 & 228200 & 30000 & 18000 & 18000 & 24000 & 30000 & 228200 & 6100 & 24100 \\
\hline 40 & 8200 & 228200 & 0 & 6000 & 228200 & 228200 & 12000 & 18000 & 24000 & 228200 & 228200 & 30000 & 18000 & 18000 & 24000 & 30000 & 228200 & 228200 & 24100 \\
\hline 50 & 228200 & 228200 & 228200 & 0 & 228200 & 228200 & 6000 & 12000 & 18000 & 228200 & 228200 & 24000 & 12000 & 12000 & 18000 & 24000 & 228200 & 228200 & 18100 \\
\hline 60 & 228200 & 228200 & 228200 & 228200 & 0 & 228200 & 60000 & 6000 & 12000 & 228200 & 228200 & 24000 & 12000 & 12000 & 18000 & 24000 & 228200 & $\begin{array}{lll}1 & 0 & 0\end{array}$ & 18100 \\
\hline 70 & 228200 & 9000 & 15000 & 18000 & 60000 & 0 & 12000 & 6000 & 60000 & 228200 & 228200 & 18000 & 18000 & 12000 & 12000 & 24000 & 228200 & 6100 & 12100 \\
\hline 80 & 228200 & 228200 & 228200 & 228200 & 228200 & 228200 & 0 & 6000 & 12000 & 228200 & 228200 & 18000 & 6000 & 6000 & 12000 & 18000 & 228200 & 228200 & 12100 \\
\hline 90 & 228200 & 228200 & 228200 & 228200 & 228200 & 228200 & 60000 & 0 & 6000 & 228200 & 228200 & 18000 & 12000 & 6000 & 12000 & 24000 & 228200 & 228200 & 12100 \\
\hline 100 & 228200 & 228200 & 228200 & 228200 & 228200 & 228200 & 12000 & 6000 & 0 & 228200 & 228200 & 12000 & 12000 & 60000 & 6000 & 18000 & 228200 & 228200 & 6100 \\
\hline 110 & 228200 & 228200 & 228200 & 228200 & 228200 & 228200 & 18000 & 12000 & 6000 & 0 & 6000 & 12000 & 12000 & 12000 & 6000 & 18000 & 228200 & 228200 & 6100 \\
\hline 120 & 228200 & 228200 & 228200 & 228200 & 228200 & 228200 & 24000 & 24000 & 18000 & 228200 & 0 & 6000 & 18000 & 18000 & 12000 & 12000 & 228200 & 228200 & 12100 \\
\hline 130 & 228200 & 228200 & 228200 & 228200 & 228200 & 228200 & 18000 & 18000 & 12000 & & 228200 & 0 & 12000 & 12000 & 6000 & 6000 & 228200 & 228200 & 6100 \\
\hline 140 & 228200 & 228200 & 228200 & 228200 & 228200 & 228200 & 18000 & 18000 & 12000 & 228200 & 228200 & 12000 & 0 & 12000 & 6000 & 12000 & 228200 & 228200 & 6100 \\
\hline 150 & 228200 & 228200 & 228200 & 228200 & 228200 & 228200 & 6000 & 6000 & 12000 & 228200 & 228200 & 12000 & 60000 & 0 & 6000 & 18000 & 228200 & 228200 & 6100 \\
\hline 160 & 228200 & 228200 & 228200 & 228200 & 228200 & 228200 & 12000 & 12000 & 6000 & 228200 & 228200 & 6000 & 6000 & 6000 & 0 & 12000 & 228200 & 228200 & $\begin{array}{lll}10 & 0 & 0\end{array}$ \\
\hline 170 & 228200 & 228200 & 228200 & 228200 & 228200 & 228200 & 24000 & 24000 & 18000 & 228200 & 228200 & 6000 & 18000 & 18000 & 12000 & 0 & 228200 & 228200 & 12100 \\
\hline 10 & 0 & 12000 & 18000 & 21000 & 18000 & 12000 & 24000 & 18000 & 18000 & 12000 & 18000 & 24000 & 24000 & 24000 & 18000 & 30000 & 0 & 18100 & 18100 \\
\hline 65 & 228200 & 228200 & 228200 & 228200 & $\begin{array}{lll}1 & 0 & 0\end{array}$ & 228200 & 6100 & 6100 & 12100 & 228200 & 228200 & 24100 & 12100 & 12100 & 18100 & 24100 & 228200 & 0 & 18200 \\
\hline 165 & 228200 & 228200 & 228200 & 228200 & 228200 & 228200 & 12100 & 12100 & 6100 & 228200 & 228200 & 6100 & 6100 & 6100 & $\begin{array}{lll}1 & 0 & 0\end{array}$ & 12100 & 228200 & 228200 & 0 \\
\hline
\end{tabular}


Estudo da Localização Otimizada de Equipamentos em Rede de Distribuição de Água para Deteç̧ão de Contaminação

Matriz Roteamento Final:

\begin{tabular}{|c|c|c|c|c|c|c|c|c|c|c|c|c|c|c|c|c|c|c|c|}
\hline Nós & 20 & 30 & 40 & 50 & 60 & 70 & 80 & 90 & 100 & 110 & 120 & 130 & 140 & 150 & 160 & 170 & 10 & 65 & 165 \\
\hline 20 & 1 & 2 & 2 & 2 & 6 & 6 & 6 & 6 & 6 & 10 & 10 & 10 & 6 & 6 & 10 & 10 & 0 & 6 & 10 \\
\hline 30 & 0 & 2 & 3 & 4 & 5 & 0 & 5 & 5 & 5 & 0 & 0 & 5 & 5 & 5 & 5 & 5 & 0 & 5 & 5 \\
\hline 40 & 0 & 0 & 3 & 4 & 0 & 0 & 4 & 4 & 4 & 0 & 0 & 4 & 4 & 4 & 4 & 4 & 0 & 0 & 4 \\
\hline 50 & 0 & 0 & 0 & 4 & 0 & 0 & 7 & 7 & 7 & 0 & 0 & 7 & 7 & 7 & 7 & 7 & 0 & 0 & 7 \\
\hline 60 & 0 & 0 & 0 & 0 & 5 & 0 & 7 & 8 & 7 & 0 & 0 & 7 & 7 & 7 & 7 & 7 & 0 & 18 & 7 \\
\hline 70 & 0 & 2 & 2 & 2 & 5 & 6 & 5 & 8 & 9 & 0 & 0 & 9 & 5 & 9 & 9 & 5 & 0 & 5 & 9 \\
\hline 80 & 0 & 0 & 0 & 0 & 0 & 0 & 7 & 8 & 13 & 0 & 0 & 13 & 13 & 14 & 13 & 13 & 0 & 0 & 13 \\
\hline 90 & 0 & 0 & 0 & 0 & 0 & 0 & 7 & 8 & 14 & 0 & 0 & 14 & 14 & 14 & 14 & 14 & 0 & 0 & 14 \\
\hline 100 & 0 & 0 & 0 & 0 & 0 & 0 & 8 & 8 & 9 & 0 & 0 & 15 & 14 & 14 & 15 & 15 & 0 & 0 & 15 \\
\hline 110 & 0 & 0 & 0 & 0 & 0 & 0 & 9 & 9 & 9 & 10 & 11 & 15 & 9 & 9 & 15 & 15 & 0 & 0 & 15 \\
\hline 120 & 0 & 0 & 0 & 0 & 0 & 0 & 12 & 12 & 12 & 0 & 11 & 12 & 12 & 12 & 12 & 12 & 0 & 0 & 12 \\
\hline 130 & 0 & 0 & 0 & 0 & 0 & 0 & 15 & 15 & 15 & 0 & 0 & 12 & 15 & 15 & 15 & 16 & 0 & 0 & 15 \\
\hline 140 & 0 & 0 & 0 & 0 & 0 & 0 & 15 & 15 & 15 & 0 & 0 & 15 & 13 & 15 & 15 & 16 & 0 & 0 & 15 \\
\hline 150 & 0 & 0 & 0 & 0 & 0 & 0 & 8 & 8 & 15 & 0 & 0 & 15 & 13 & 14 & 15 & 13 & 0 & 0 & 15 \\
\hline 160 & 0 & 0 & 0 & 0 & 0 & 0 & 14 & 14 & 9 & 0 & 0 & 12 & 13 & 14 & 15 & 12 & 0 & 0 & 19 \\
\hline 170 & 0 & 0 & 0 & 0 & 0 & 0 & 12 & 12 & 12 & 0 & 0 & 12 & 12 & 12 & 12 & 16 & 0 & 0 & 12 \\
\hline 10 & 1 & 1 & 1 & 1 & 1 & 1 & 1 & 1 & 1 & 1 & 1 & 1 & 1 & 1 & 1 & 1 & 17 & 1 & 1 \\
\hline 65 & 0 & 0 & 0 & 0 & 5 & 0 & 5 & 5 & 5 & 0 & 0 & 5 & 5 & 5 & 5 & 5 & 0 & 18 & 5 \\
\hline 165 & 0 & 0 & 0 & 0 & 0 & 0 & 15 & 15 & 15 & 0 & 0 & 15 & 15 & 15 & 15 & 15 & 0 & 0 & 19 \\
\hline
\end{tabular}

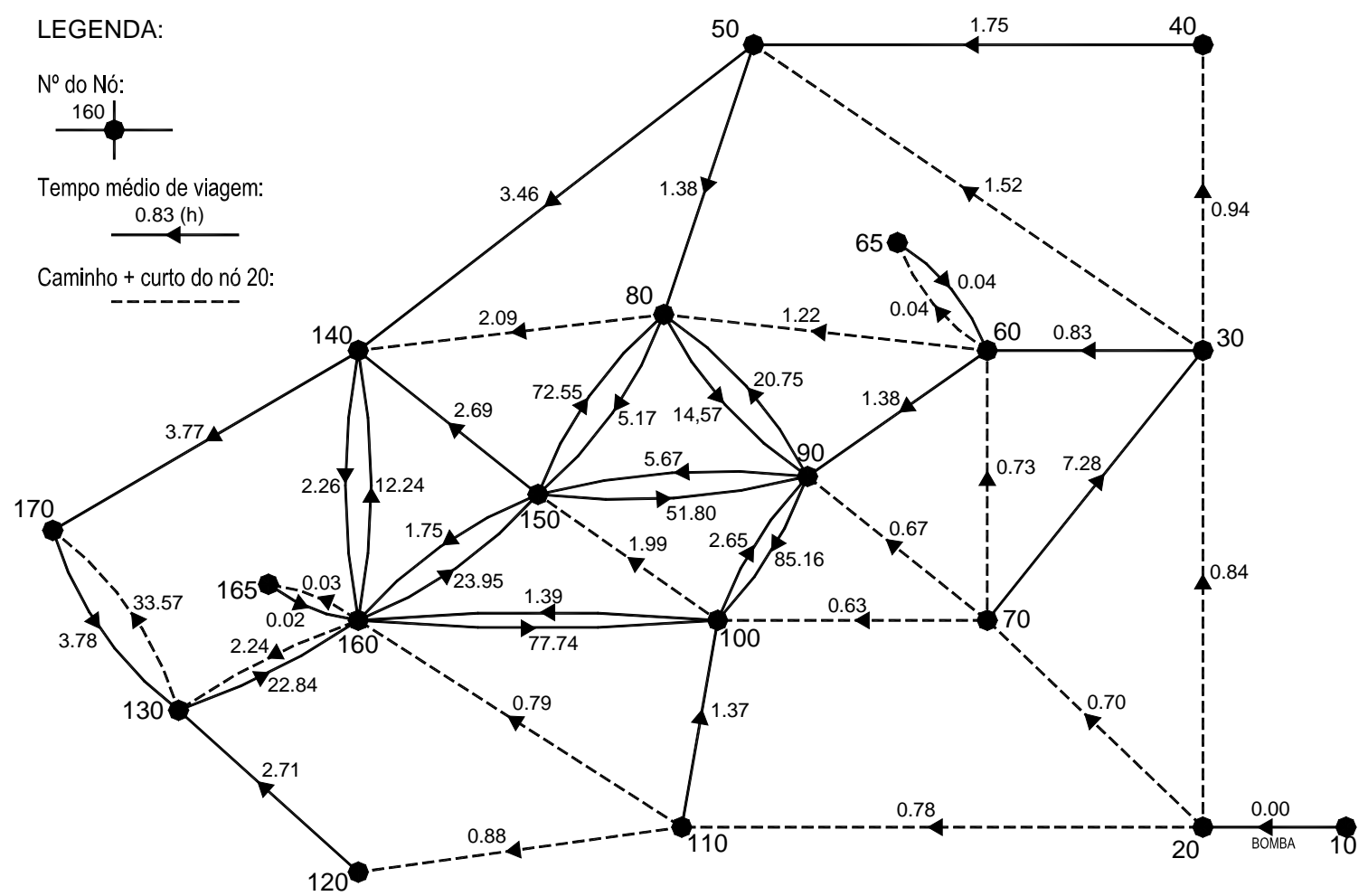

Figura 6.2 - Rede auxiliar para Tempo de viagem da Rede Anytown 


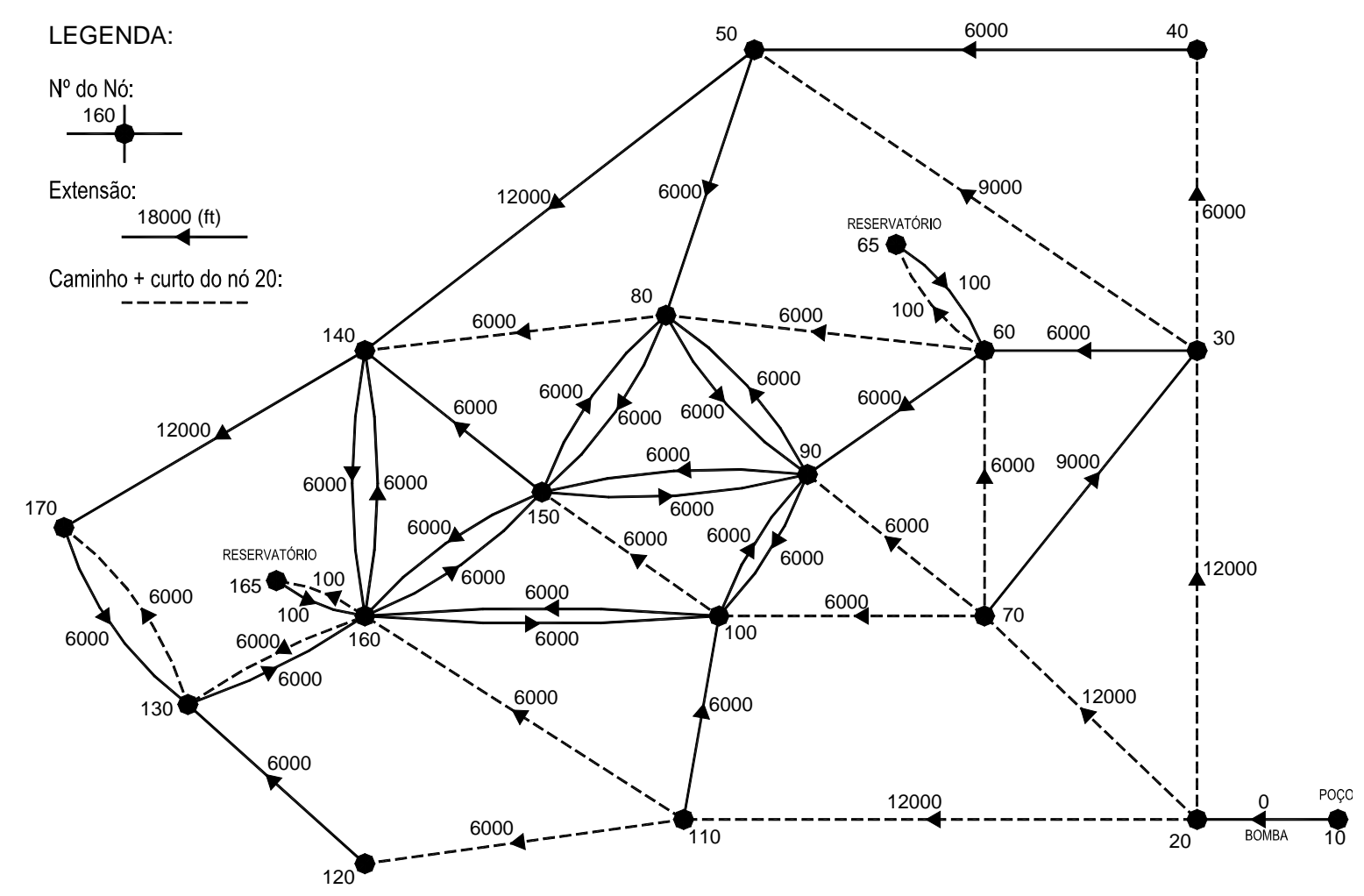

Figura 6.3 - Rede auxiliar para Extensão de viagem da Rede Anytown

\subsection{Determinação das Matrizes de Poluição}

As Matrizes de Poluição construídas são apresentadas a seguir. 
Estudo da Localização Otimizada de Equipamentos em Rede de Distribuição de Água para Deteç̧ão de Contaminação

Matriz Poluição para Volume Consumido (nível de serviço: 468.10m3):

\begin{tabular}{|c|c|c|c|c|c|c|c|c|c|c|c|c|c|c|c|c|c|c|c|}
\hline Nós & $\mathbf{2 0}$ & $\mathbf{3 0}$ & $\mathbf{4 0}$ & $\mathbf{5 0}$ & $\mathbf{6 0}$ & $\mathbf{7 0}$ & $\mathbf{8 0}$ & $\mathbf{9 0}$ & $\mathbf{1 0 0}$ & $\mathbf{1 1 0}$ & $\mathbf{1 2 0}$ & $\mathbf{1 3 0}$ & $\mathbf{1 4 0}$ & $\mathbf{1 5 0}$ & $\mathbf{1 6 0}$ & $\mathbf{1 7 0}$ & $\mathbf{1 0}$ & $\mathbf{6 5}$ & $\mathbf{1 6 5}$ \\
\hline $\mathbf{2 0}$ & 1 & 1 & 0 & 0 & 1 & 1 & 0 & 1 & 1 & 1 & 0 & 0 & 0 & 0 & 0 & 0 & 0 & 1 & 0 \\
\hline $\mathbf{3 0}$ & 0 & 1 & 1 & 1 & 1 & 0 & 1 & 1 & 0 & 0 & 0 & 0 & 0 & 0 & 0 & 0 & 0 & 1 & 0 \\
\hline $\mathbf{4 0}$ & 0 & 0 & 1 & 1 & 0 & 0 & 1 & 0 & 0 & 0 & 0 & 0 & 0 & 0 & 0 & 0 & 0 & 0 & 0 \\
\hline $\mathbf{5 0}$ & 0 & 0 & 0 & 1 & 0 & 0 & 1 & 0 & 0 & 0 & 0 & 0 & 1 & 0 & 0 & 0 & 0 & 0 & 0 \\
\hline $\mathbf{6 0}$ & 0 & 0 & 0 & 0 & 1 & 0 & 1 & 1 & 0 & 0 & 0 & 0 & 0 & 0 & 0 & 0 & 0 & 1 & 0 \\
\hline $\mathbf{7 0}$ & 0 & 0 & 0 & 0 & 1 & 1 & 0 & 1 & 1 & 0 & 0 & 0 & 0 & 0 & 0 & 0 & 0 & 1 & 0 \\
\hline $\mathbf{8 0}$ & 0 & 0 & 0 & 0 & 0 & 0 & 1 & 0 & 0 & 0 & 0 & 0 & 1 & 0 & 0 & 0 & 0 & 0 & 0 \\
\hline $\mathbf{9 0}$ & 0 & 0 & 0 & 0 & 0 & 0 & 0 & 1 & 0 & 0 & 0 & 0 & 0 & 0 & 0 & 0 & 0 & 0 & 0 \\
\hline $\mathbf{1 0 0}$ & 0 & 0 & 0 & 0 & 0 & 0 & 0 & 0 & 1 & 0 & 0 & 0 & 0 & 1 & 1 & 0 & 0 & 0 & 1 \\
\hline $\mathbf{1 1 0}$ & 0 & 0 & 0 & 0 & 0 & 0 & 0 & 0 & 1 & 1 & 1 & 0 & 0 & 0 & 1 & 0 & 0 & 0 & 1 \\
\hline $\mathbf{1 2 0}$ & 0 & 0 & 0 & 0 & 0 & 0 & 0 & 0 & 0 & 0 & 1 & 1 & 0 & 0 & 0 & 1 & 0 & 0 & 0 \\
\hline $\mathbf{1 3 0}$ & 0 & 0 & 0 & 0 & 0 & 0 & 0 & 0 & 0 & 0 & 0 & 1 & 0 & 0 & 0 & 1 & 0 & 0 & 0 \\
\hline $\mathbf{1 4 0}$ & 0 & 0 & 0 & 0 & 0 & 0 & 0 & 0 & 0 & 0 & 0 & 0 & 1 & 0 & 1 & 1 & 0 & 0 & 1 \\
\hline $\mathbf{1 5 0}$ & 0 & 0 & 0 & 0 & 0 & 0 & 0 & 0 & 0 & 0 & 0 & 0 & 1 & 1 & 1 & 0 & 0 & 0 & 1 \\
\hline $\mathbf{1 6 0}$ & 0 & 0 & 0 & 0 & 0 & 0 & 0 & 0 & 0 & 0 & 0 & 1 & 0 & 0 & 1 & 0 & 0 & 0 & 1 \\
\hline $\mathbf{1 7 0}$ & 0 & 0 & 0 & 0 & 0 & 0 & 0 & 0 & 0 & 0 & 0 & 0 & 0 & 0 & 0 & 1 & 0 & 0 & 0 \\
\hline $\mathbf{1 0}$ & 1 & 1 & 0 & 0 & 1 & 1 & 0 & 1 & 1 & 1 & 0 & 0 & 0 & 0 & 0 & 0 & 0 & 1 & 0 \\
\hline $\mathbf{6 5}$ & 0 & 0 & 0 & 0 & 1 & 0 & 1 & 1 & 0 & 0 & 0 & 0 & 0 & 0 & 0 & 0 & 0 & 0 & 0 \\
\hline $\mathbf{1 6 5}$ & 0 & 0 & 0 & 0 & 0 & 0 & 0 & 0 & 0 & 0 & 0 & 1 & 0 & 0 & 1 & 0 & 0 & 0 & 0 \\
\hline
\end{tabular}

Matriz Poluição para Volume Consumido (nível de serviço: 1245.15m3):

\begin{tabular}{|c|c|c|c|c|c|c|c|c|c|c|c|c|c|c|c|c|c|c|c|}
\hline Nós & $\mathbf{2 0}$ & $\mathbf{3 0}$ & $\mathbf{4 0}$ & $\mathbf{5 0}$ & $\mathbf{6 0}$ & $\mathbf{7 0}$ & $\mathbf{8 0}$ & $\mathbf{9 0}$ & $\mathbf{1 0 0}$ & $\mathbf{1 1 0}$ & $\mathbf{1 2 0}$ & $\mathbf{1 3 0}$ & $\mathbf{1 4 0}$ & $\mathbf{1 5 0}$ & $\mathbf{1 6 0}$ & $\mathbf{1 7 0}$ & $\mathbf{1 0}$ & $\mathbf{6 5}$ & $\mathbf{1 6 5}$ \\
\hline $\mathbf{2 0}$ & 1 & 1 & 1 & 0 & 1 & 1 & 0 & 1 & 1 & 1 & 1 & 0 & 0 & 0 & 1 & 0 & 0 & 1 & 1 \\
\hline $\mathbf{3 0}$ & 0 & 1 & 1 & 1 & 1 & 0 & 1 & 1 & 0 & 0 & 0 & 0 & 0 & 0 & 0 & 0 & 0 & 1 & 0 \\
\hline $\mathbf{4 0}$ & 0 & 0 & 1 & 1 & 0 & 0 & 1 & 0 & 0 & 0 & 0 & 0 & 1 & 0 & 1 & 0 & 0 & 0 & 1 \\
\hline $\mathbf{5 0}$ & 0 & 0 & 0 & 1 & 0 & 0 & 1 & 0 & 0 & 0 & 0 & 0 & 1 & 1 & 1 & 0 & 0 & 0 & 1 \\
\hline $\mathbf{6 0}$ & 0 & 0 & 0 & 0 & 1 & 0 & 1 & 1 & 0 & 0 & 0 & 0 & 1 & 0 & 0 & 0 & 0 & 1 & 0 \\
\hline $\mathbf{7 0}$ & 0 & 0 & 0 & 0 & 1 & 1 & 1 & 1 & 1 & 0 & 0 & 0 & 0 & 0 & 1 & 0 & 0 & 1 & 1 \\
\hline $\mathbf{8 0}$ & 0 & 0 & 0 & 0 & 0 & 0 & 1 & 0 & 0 & 0 & 0 & 0 & 1 & 1 & 1 & 1 & 0 & 0 & 1 \\
\hline $\mathbf{9 0}$ & 0 & 0 & 0 & 0 & 0 & 0 & 0 & 1 & 0 & 0 & 0 & 0 & 0 & 0 & 0 & 0 & 0 & 0 & 0 \\
\hline $\mathbf{1 0 0}$ & 0 & 0 & 0 & 0 & 0 & 0 & 0 & 1 & 1 & 0 & 0 & 1 & 0 & 1 & 1 & 0 & 0 & 0 & 1 \\
\hline $\mathbf{1 1 0}$ & 0 & 0 & 0 & 0 & 0 & 0 & 0 & 0 & 1 & 1 & 1 & 1 & 0 & 1 & 1 & 0 & 0 & 0 & 1 \\
\hline $\mathbf{1 2 0}$ & 0 & 0 & 0 & 0 & 0 & 0 & 0 & 0 & 0 & 0 & 1 & 1 & 0 & 0 & 0 & 1 & 0 & 0 & 0 \\
\hline $\mathbf{1 3 0}$ & 0 & 0 & 0 & 0 & 0 & 0 & 0 & 0 & 0 & 0 & 0 & 1 & 0 & 0 & 0 & 1 & 0 & 0 & 0 \\
\hline $\mathbf{1 4 0}$ & 0 & 0 & 0 & 0 & 0 & 0 & 0 & 0 & 0 & 0 & 0 & 1 & 1 & 0 & 1 & 1 & 0 & 0 & 1 \\
\hline $\mathbf{1 5 0}$ & 0 & 0 & 0 & 0 & 0 & 0 & 0 & 0 & 0 & 0 & 0 & 1 & 1 & 1 & 1 & 0 & 0 & 0 & 1 \\
\hline $\mathbf{1 6 0}$ & 0 & 0 & 0 & 0 & 0 & 0 & 0 & 0 & 0 & 0 & 0 & 1 & 0 & 0 & 1 & 0 & 0 & 0 & 1 \\
\hline $\mathbf{1 7 0}$ & 0 & 0 & 0 & 0 & 0 & 0 & 0 & 0 & 0 & 0 & 0 & 0 & 0 & 0 & 0 & 1 & 0 & 0 & 0 \\
\hline $\mathbf{1 0}$ & 1 & 1 & 1 & 0 & 1 & 1 & 0 & 1 & 1 & 1 & 1 & 0 & 0 & 0 & 1 & 0 & 0 & 1 & 1 \\
\hline $\mathbf{6 5}$ & 0 & 0 & 0 & 0 & 1 & 0 & 1 & 1 & 0 & 0 & 0 & 0 & 1 & 0 & 0 & 0 & 0 & 0 & 0 \\
\hline $\mathbf{1 6 5}$ & 0 & 0 & 0 & 0 & 0 & 0 & 0 & 0 & 0 & 0 & 0 & 1 & 0 & 0 & 1 & 0 & 0 & 0 & 0 \\
\hline
\end{tabular}


Estudo da Localização Otimizada de Equipamentos em Rede de Distribuição de Água para Deteç̧ão de Contaminação

Matriz Poluição para Tempo de Deteç̧ão (nível de serviço: 6.00h.)

\begin{tabular}{|c|c|c|c|c|c|c|c|c|c|c|c|c|c|c|c|c|c|c|c|}
\hline Nós & $\mathbf{2 0}$ & $\mathbf{3 0}$ & $\mathbf{4 0}$ & $\mathbf{5 0}$ & $\mathbf{6 0}$ & $\mathbf{7 0}$ & $\mathbf{8 0}$ & $\mathbf{9 0}$ & $\mathbf{1 0 0}$ & $\mathbf{1 1 0}$ & $\mathbf{1 2 0}$ & $\mathbf{1 3 0}$ & $\mathbf{1 4 0}$ & $\mathbf{1 5 0}$ & $\mathbf{1 6 0}$ & $\mathbf{1 7 0}$ & $\mathbf{1 0}$ & $\mathbf{6 5}$ & $\mathbf{1 6 5}$ \\
\hline $\mathbf{2 0}$ & 1 & 1 & 1 & 1 & 1 & 1 & 1 & 1 & 1 & 1 & 1 & 1 & 1 & 1 & 1 & 0 & 0 & 1 & 1 \\
\hline $\mathbf{3 0}$ & 0 & 1 & 1 & 1 & 1 & 0 & 1 & 1 & 0 & 0 & 0 & 0 & 1 & 0 & 0 & 0 & 0 & 1 & 0 \\
\hline $\mathbf{4 0}$ & 0 & 0 & 1 & 1 & 0 & 0 & 1 & 0 & 0 & 0 & 0 & 0 & 1 & 0 & 0 & 0 & 0 & 0 & 0 \\
\hline $\mathbf{5 0}$ & 0 & 0 & 0 & 1 & 0 & 0 & 1 & 0 & 0 & 0 & 0 & 0 & 1 & 0 & 1 & 0 & 0 & 0 & 1 \\
\hline $\mathbf{6 0}$ & 0 & 0 & 0 & 0 & 1 & 0 & 1 & 1 & 0 & 0 & 0 & 0 & 1 & 0 & 1 & 0 & 0 & 1 & 1 \\
\hline $\mathbf{7 0}$ & 0 & 0 & 0 & 0 & 1 & 1 & 1 & 1 & 1 & 0 & 0 & 1 & 1 & 1 & 1 & 0 & 0 & 1 & 1 \\
\hline $\mathbf{8 0}$ & 0 & 0 & 0 & 0 & 0 & 0 & 1 & 0 & 0 & 0 & 0 & 0 & 1 & 1 & 1 & 1 & 0 & 0 & 1 \\
\hline $\mathbf{9 0}$ & 0 & 0 & 0 & 0 & 0 & 0 & 0 & 1 & 0 & 0 & 0 & 0 & 0 & 1 & 0 & 0 & 0 & 0 & 0 \\
\hline $\mathbf{1 0 0}$ & 0 & 0 & 0 & 0 & 0 & 0 & 0 & 1 & 1 & 0 & 0 & 1 & 1 & 1 & 1 & 0 & 0 & 0 & 1 \\
\hline $\mathbf{1 1 0}$ & 0 & 0 & 0 & 0 & 0 & 0 & 0 & 1 & 1 & 1 & 1 & 1 & 0 & 1 & 1 & 0 & 0 & 0 & 1 \\
\hline $\mathbf{1 2 0}$ & 0 & 0 & 0 & 0 & 0 & 0 & 0 & 0 & 0 & 0 & 1 & 1 & 0 & 0 & 0 & 0 & 0 & 0 & 0 \\
\hline $\mathbf{1 3 0}$ & 0 & 0 & 0 & 0 & 0 & 0 & 0 & 0 & 0 & 0 & 0 & 1 & 0 & 0 & 0 & 1 & 0 & 0 & 0 \\
\hline $\mathbf{1 4 0}$ & 0 & 0 & 0 & 0 & 0 & 0 & 0 & 0 & 0 & 0 & 0 & 1 & 1 & 0 & 1 & 1 & 0 & 0 & 1 \\
\hline $\mathbf{1 5 0}$ & 0 & 0 & 0 & 0 & 0 & 0 & 0 & 0 & 0 & 0 & 0 & 1 & 1 & 1 & 1 & 0 & 0 & 0 & 1 \\
\hline $\mathbf{1 6 0}$ & 0 & 0 & 0 & 0 & 0 & 0 & 0 & 0 & 0 & 0 & 0 & 1 & 0 & 0 & 1 & 0 & 0 & 0 & 1 \\
\hline $\mathbf{1 7 0}$ & 0 & 0 & 0 & 0 & 0 & 0 & 0 & 0 & 0 & 0 & 0 & 0 & 0 & 0 & 0 & 1 & 0 & 0 & 0 \\
\hline $\mathbf{1 0}$ & 1 & 1 & 1 & 1 & 1 & 1 & 1 & 1 & 1 & 1 & 1 & 1 & 1 & 1 & 1 & 0 & 1 & 1 & 1 \\
\hline $\mathbf{6 5}$ & 0 & 0 & 0 & 0 & 1 & 0 & 1 & 1 & 0 & 0 & 0 & 0 & 1 & 0 & 1 & 0 & 0 & 1 & 1 \\
\hline $\mathbf{1 6 5}$ & 0 & 0 & 0 & 0 & 0 & 0 & 0 & 0 & 0 & 0 & 0 & 1 & 0 & 0 & 1 & 0 & 0 & 0 & 1 \\
\hline
\end{tabular}

Matriz Poluição para Extensão da Contaminação (nível de serviço: 15.00\%):

\begin{tabular}{|c|c|c|c|c|c|c|c|c|c|c|c|c|c|c|c|c|c|c|c|}
\hline Nós & $\mathbf{2 0}$ & $\mathbf{3 0}$ & $\mathbf{4 0}$ & $\mathbf{5 0}$ & $\mathbf{6 0}$ & $\mathbf{7 0}$ & $\mathbf{8 0}$ & $\mathbf{9 0}$ & $\mathbf{1 0 0}$ & $\mathbf{1 1 0}$ & $\mathbf{1 2 0}$ & $\mathbf{1 3 0}$ & $\mathbf{1 4 0}$ & $\mathbf{1 5 0}$ & $\mathbf{1 6 0}$ & $\mathbf{1 7 0}$ & $\mathbf{1 0}$ & $\mathbf{6 5}$ & $\mathbf{1 6 5}$ \\
\hline $\mathbf{2 0}$ & 1 & 1 & 1 & 1 & 1 & 1 & 1 & 1 & 1 & 1 & 1 & 1 & 1 & 1 & 1 & 1 & 0 & 1 & 1 \\
\hline $\mathbf{3 0}$ & 0 & 1 & 1 & 1 & 1 & 0 & 1 & 1 & 1 & 0 & 0 & 1 & 1 & 1 & 1 & 1 & 0 & 1 & 1 \\
\hline $\mathbf{4 0}$ & 0 & 0 & 1 & 1 & 0 & 0 & 1 & 1 & 1 & 0 & 0 & 1 & 1 & 1 & 1 & 1 & 0 & 0 & 1 \\
\hline $\mathbf{5 0}$ & 0 & 0 & 0 & 1 & 0 & 0 & 1 & 1 & 1 & 0 & 0 & 1 & 1 & 1 & 1 & 1 & 0 & 0 & 1 \\
\hline $\mathbf{6 0}$ & 0 & 0 & 0 & 0 & 1 & 0 & 1 & 1 & 1 & 0 & 0 & 1 & 1 & 1 & 1 & 1 & 0 & 1 & 1 \\
\hline $\mathbf{7 0}$ & 0 & 1 & 1 & 1 & 1 & 1 & 1 & 1 & 1 & 0 & 0 & 1 & 1 & 1 & 1 & 1 & 0 & 1 & 1 \\
\hline $\mathbf{8 0}$ & 0 & 0 & 0 & 0 & 0 & 0 & 1 & 1 & 1 & 0 & 0 & 1 & 1 & 1 & 1 & 1 & 0 & 0 & 1 \\
\hline $\mathbf{9 0}$ & 0 & 0 & 0 & 0 & 0 & 0 & 1 & 1 & 1 & 0 & 0 & 1 & 1 & 1 & 1 & 1 & 0 & 0 & 1 \\
\hline $\mathbf{1 0 0}$ & 0 & 0 & 0 & 0 & 0 & 0 & 1 & 1 & 1 & 0 & 0 & 1 & 1 & 1 & 1 & 1 & 0 & 0 & 1 \\
\hline $\mathbf{1 1 0}$ & 0 & 0 & 0 & 0 & 0 & 0 & 1 & 1 & 1 & 1 & 1 & 1 & 1 & 1 & 1 & 1 & 0 & 0 & 1 \\
\hline $\mathbf{1 2 0}$ & 0 & 0 & 0 & 0 & 0 & 0 & 1 & 1 & 1 & 0 & 1 & 1 & 1 & 1 & 1 & 1 & 0 & 0 & 1 \\
\hline $\mathbf{1 3 0}$ & 0 & 0 & 0 & 0 & 0 & 0 & 1 & 1 & 1 & 0 & 0 & 1 & 1 & 1 & 1 & 1 & 0 & 0 & 1 \\
\hline $\mathbf{1 4 0}$ & 0 & 0 & 0 & 0 & 0 & 0 & 1 & 1 & 1 & 0 & 0 & 1 & 1 & 1 & 1 & 1 & 0 & 0 & 1 \\
\hline $\mathbf{1 5 0}$ & 0 & 0 & 0 & 0 & 0 & 0 & 1 & 1 & 1 & 0 & 0 & 1 & 1 & 1 & 1 & 1 & 0 & 0 & 1 \\
\hline $\mathbf{1 6 0}$ & 0 & 0 & 0 & 0 & 0 & 0 & 1 & 1 & 1 & 0 & 0 & 1 & 1 & 1 & 1 & 1 & 0 & 0 & 1 \\
\hline $\mathbf{1 7 0}$ & 0 & 0 & 0 & 0 & 0 & 0 & 1 & 1 & 1 & 0 & 0 & 1 & 1 & 1 & 1 & 1 & 0 & 0 & 1 \\
\hline $\mathbf{1 0}$ & 1 & 1 & 1 & 1 & 1 & 1 & 1 & 1 & 1 & 1 & 1 & 1 & 1 & 1 & 1 & 1 & 1 & 1 & 1 \\
\hline $\mathbf{6 5}$ & 0 & 0 & 0 & 0 & 1 & 0 & 1 & 1 & 1 & 0 & 0 & 1 & 1 & 1 & 1 & 1 & 0 & 1 & 1 \\
\hline $\mathbf{1 6 5}$ & 0 & 0 & 0 & 0 & 0 & 0 & 1 & 1 & 1 & 0 & 0 & 1 & 1 & 1 & 1 & 1 & 0 & 0 & 1 \\
\hline
\end{tabular}




\subsection{Inicialização do Programa PLEDECORDA_MOM}

O programa denominado PLEDECORDA_MOM importou de um arquivo .INP, o número de nós da rede e as matrizes poluição e solicitou mais dois dados, o número de equipamentos, considerado de 1 a 4 unidades, e a consideração ou não de acréscimo por nó já monitorado. Como resultado, o programa exportou o arquivo PLEDECORDA_MOM.TXT, com a Frente de Pareto ou conjunto de soluções não-dominadas identificado, conforme os anexos VI e VIII (arquivo PLEDECORDA_MOM.TXT para a Rede Anytown).

\subsection{Determinação dos Nós de Monitoramento}

No caso da Rede Anytown., as tabelas 6.6 e 6.7 apresentam as soluções geradas pelo programa PLEDECORDA_MOM para $\mathrm{VC}=468,10 \mathrm{~m}^{3}$, e as tabelas 6.8 e 6.9 apresentam as soluções geradas pelo programa PLEDECORDA_MOM para $\mathrm{VC}=1245,15 \mathrm{~m}^{3}$. 
Tabela 6.6 - Resultados da Rede Anytown não considerando detecções repetidas: Nível $\mathrm{VC}=468,10 \mathrm{~m}^{3}$.

\begin{tabular}{|c|c|c|c|c|}
\hline \multicolumn{5}{|c|}{ No de equipamentos para monitoramento: 01un. } \\
\hline Solução & \multicolumn{3}{|c|}{ no espaço de objetivos } & no espaço de decisão \\
\hline $\mathrm{N}^{\mathrm{o}}$. & F1 & $\mathrm{F} 2$ & F3 & Nó de monitoramento \\
\hline 1 & 6 & 13 & 19 & 160 \\
\hline 2 & 7 & 9 & 19 & 90 \\
\hline \multicolumn{5}{|c|}{ No de equipamentos para monitoramento: 02un. } \\
\hline Solução & \multicolumn{3}{|c|}{ no espaço de objetivos } & no espaço de decisão \\
\hline $\mathrm{N}^{\mathrm{o}}$. & $\mathrm{F} 1$ & $\mathrm{~F} 2$ & F3 & Nós de monitoramento \\
\hline 1 & 13 & 15 & 19 & 160 e 90 \\
\hline 2 & 10 & 17 & 19 & 130 e 80 \\
\hline \multicolumn{5}{|c|}{ No de equipamentos para monitoramento: 03un. } \\
\hline Solução & \multicolumn{3}{|c|}{ no espaço de objetivos } & no espaço de decisão \\
\hline $\mathrm{N}^{\mathrm{o}}$. & F1 & $\mathrm{F} 2$ & F3 & Nós de monitoramento \\
\hline 1 & 15 & 18 & 19 & 140,90 e 130 \\
\hline 2 & 16 & 17 & 19 & 90,160 e 170 \\
\hline \multicolumn{5}{|c|}{ No de equipamentos para monitoramento: 04un. } \\
\hline Solução & \multicolumn{3}{|c|}{ no espaço de objetivos } & no espaço de decisão \\
\hline $\mathrm{N}^{\mathrm{o}}$. & F1 & $\mathrm{F} 2$ & F3 & Nós de monitoramento \\
\hline 1 & 16 & 19 & 19 & $130,170,90$ e 80 \\
\hline 2 & 19 & 18 & 19 & $90,160,170$ e 80 \\
\hline
\end{tabular}


Tabela 6.7 - Resultados da Rede Anytown considerando detecções repetidas: Nível VC $=468,10 \mathrm{~m}^{3}$.

\begin{tabular}{|c|c|c|c|c|}
\hline \multicolumn{5}{|c|}{$\mathrm{N}^{\mathrm{o}}$ de equipamentos para monitoramento: $01 \mathrm{un}$. } \\
\hline Solução & \multicolumn{3}{|c|}{ no espaço de objetivos } & no espaço de decisão \\
\hline $\mathrm{N}^{\mathrm{o}}$. & F1 & $\mathrm{F} 2$ & F3 & Nó de monitoramento \\
\hline 1 & 7 & 9 & 19 & 90 \\
\hline 2 & 6 & 13 & 19 & 160 \\
\hline \multicolumn{5}{|c|}{$\mathrm{N}^{0}$ de equipamentos para monitoramento: $02 \mathrm{un}$. } \\
\hline Solução & \multicolumn{3}{|c|}{ no espaço de objetivos } & no espaço de decisão \\
\hline $\mathrm{N}^{\mathrm{o}}$. & F1 & $\mathrm{F} 2$ & F3 & Nós de monitoramento \\
\hline 1 & 13 & 15.07 & 19.19 & 90,160 \\
\hline 2 & 8 & 17.06 & 19.19 & 130,140 \\
\hline 3 & 10 & 17.03 & 19.19 & 80,130 \\
\hline \multicolumn{5}{|c|}{$\mathrm{N}^{\circ}$ de equipamentos para monitoramento: 03un. } \\
\hline Solução & \multicolumn{3}{|c|}{ no espaço de objetivos } & no espaço de decisão \\
\hline $\mathrm{N}^{\mathrm{o}}$. & F1 & $\mathrm{F} 2$ & $\mathrm{~F} 3$ & Nós de monitoramento \\
\hline 1 & 15 & 18.14 & 19.38 & $130,90,140$ \\
\hline 2 & 16.03 & 16.15 & 19.38 & $160,80,90$ \\
\hline 3 & 16.01 & 17.09 & 19.38 & $160,90,170$ \\
\hline 4 & 15.02 & 17.16 & 19.38 & $160,90,130$ \\
\hline \multicolumn{5}{|c|}{$\mathrm{N}^{\circ}$ de equipamentos para monitoramento: 04un. } \\
\hline Solução & \multicolumn{3}{|c|}{ no espaço de objetivos } & no espaço de decisão \\
\hline $\mathrm{N}^{\mathrm{o}}$. & $\mathrm{F} 1$ & $\mathrm{~F} 2$ & $\mathrm{~F} 3$ & Nós de monitoramento \\
\hline 1 & 16.05 & 19.14 & 19.57 & $130,80,90,170$ \\
\hline 2 & 17.04 & 18.27 & 19.57 & $130,90,140,160$ \\
\hline 3 & 18.05 & 18.24 & 19.57 & $130,80,90,160$ \\
\hline 4 & 16.03 & 19.17 & 19.57 & $130,90,140,170$ \\
\hline 5 & 19,04 & 18.17 & 19.57 & $160,80,90,170$ \\
\hline
\end{tabular}


Tabela 6.8 - Resultados da Rede Anytown não considerando detecções repetidas: Nível VC $=1245,15 \mathrm{~m}^{3}$.

\begin{tabular}{|c|c|c|c|c|}
\hline \multicolumn{5}{|c|}{$\mathbf{N}^{0}$ de equipamentos para monitoramento: 01un. } \\
\hline Solução & \multicolumn{3}{|c|}{ no espaço de objetivos } & no espaço de decisão \\
\hline $\mathrm{N}^{\mathrm{o}}$. & F1 & $\mathrm{F} 2$ & F3 & Nós de monitoramento \\
\hline 1 & 12 & 13 & 19 & 160 \\
\hline \multicolumn{5}{|c|}{$\mathrm{N}^{\circ}$ de equipamentos para monitoramento: $02 \mathrm{un}$. } \\
\hline Solução & \multicolumn{3}{|c|}{ no espaço de objetivos } & no espaço de decisão \\
\hline $\mathrm{N}^{\mathrm{o}}$. & F1 & $\mathrm{F} 2$ & F3 & Nós de monitoramento \\
\hline 1 & 15 & 17 & 19 & 80,130 \\
\hline 2 & 16 & 15 & 19 & 90,160 \\
\hline \multicolumn{5}{|c|}{$\mathrm{N}^{\circ}$ de equipamentos para monitoramento: 03un. } \\
\hline Solução & \multicolumn{3}{|c|}{ no espaço de objetivos } & no espaço de decisão \\
\hline $\mathrm{N}^{\mathrm{o}}$. & $\mathrm{F} 1$ & $\mathrm{~F} 2$ & F3 & Nós de monitoramento \\
\hline 1 & 19 & 17 & 19 & $90,160,170$ \\
\hline 2 & 18 & 18 & 19 & $80,130,90$ \\
\hline \multicolumn{5}{|c|}{$\mathrm{N}^{\circ}$ de equipamentos para monitoramento: 04un. } \\
\hline Solução & \multicolumn{3}{|c|}{ no espaço de objetivos } & no espaço de decisão \\
\hline $\mathrm{N}^{\mathrm{o}}$. & $\mathrm{F} 1$ & $\mathrm{~F} 2$ & F3 & Nós de monitoramento \\
\hline 1 & 19 & 19 & 19 & $170,130,80,90$ \\
\hline
\end{tabular}


Tabela 6.9 - Resultados da Rede Anytown considerando detecções repetidas: Nível VC $=1245,15 \mathrm{~m}^{3}$.

\begin{tabular}{|c|c|c|c|c|}
\hline \multicolumn{5}{|c|}{$\mathrm{N}^{\circ}$ de equipamentos para monitoramento: 01un. } \\
\hline Solução & \multicolumn{3}{|c|}{ no espaço de objetivos } & no espaço de decisão \\
\hline $\mathrm{N}^{\mathrm{o}}$. & F1 & $\mathrm{F} 2$ & F3 & Nó de monitoramento \\
\hline 1 & 12 & 13 & 19 & 160 \\
\hline \multicolumn{5}{|c|}{$\mathrm{N}^{\circ}$ de equipamentos para monitoramento: $02 \mathrm{un}$. } \\
\hline Solução & \multicolumn{3}{|c|}{ no espaço de objetivos } & no espaço de decisão \\
\hline $\mathrm{N}^{\mathrm{o}}$. & $\mathrm{F} 1$ & $\mathrm{~F} 2$ & F3 & Nós de monitoramento \\
\hline 1 & 16.04 & 15.07 & 19.19 & 160,90 \\
\hline 2 & 13.02 & 17.06 & 19.19 & 140,130 \\
\hline 3 & 15 & 17.03 & 19.19 & 130,80 \\
\hline \multicolumn{5}{|c|}{$\mathrm{N}^{\circ}$ de equipamentos para monitoramento: 03un. } \\
\hline Solução & \multicolumn{3}{|c|}{ no espaço de objetivos } & no espaço de decisão \\
\hline $\mathrm{N}^{\mathrm{o}}$. & $\mathrm{F} 1$ & $\mathrm{~F} 2$ & F3 & Nós de monitoramento \\
\hline 1 & 18.05 & 18.14 & 19.38 & $130,140,90$ \\
\hline 2 & 18.01 & 17.16 & 19.38 & $130,160,90$ \\
\hline 3 & 19.06 & 17.09 & 19.38 & $170,160,90$ \\
\hline \multicolumn{5}{|c|}{$\mathrm{N}^{\circ}$ de equipamentos para monitoramento: 04un. } \\
\hline Solução & \multicolumn{3}{|c|}{ no espaço de objetivos } & no espaço de decisão \\
\hline $\mathrm{N}^{\mathrm{o}}$. & F1 & $\mathrm{F} 2$ & F3 & Nós de monitoramento \\
\hline 1 & 19.13 & 18.2 & 19.57 & $160,140,170,90$ \\
\hline 2 & 19.09 & 19.17 & 19.57 & $140,130,170,90$ \\
\hline 3 & 19.14 & 18.19 & 19.57 & $160,130,170,90$ \\
\hline 4 & 19.17 & 17.22 & 19.57 & $160,165,170,90$ \\
\hline
\end{tabular}

Para visualização dos resultados na rede, são apresentadas as

figuras a seguir, referentes à tabela 6.9 . 


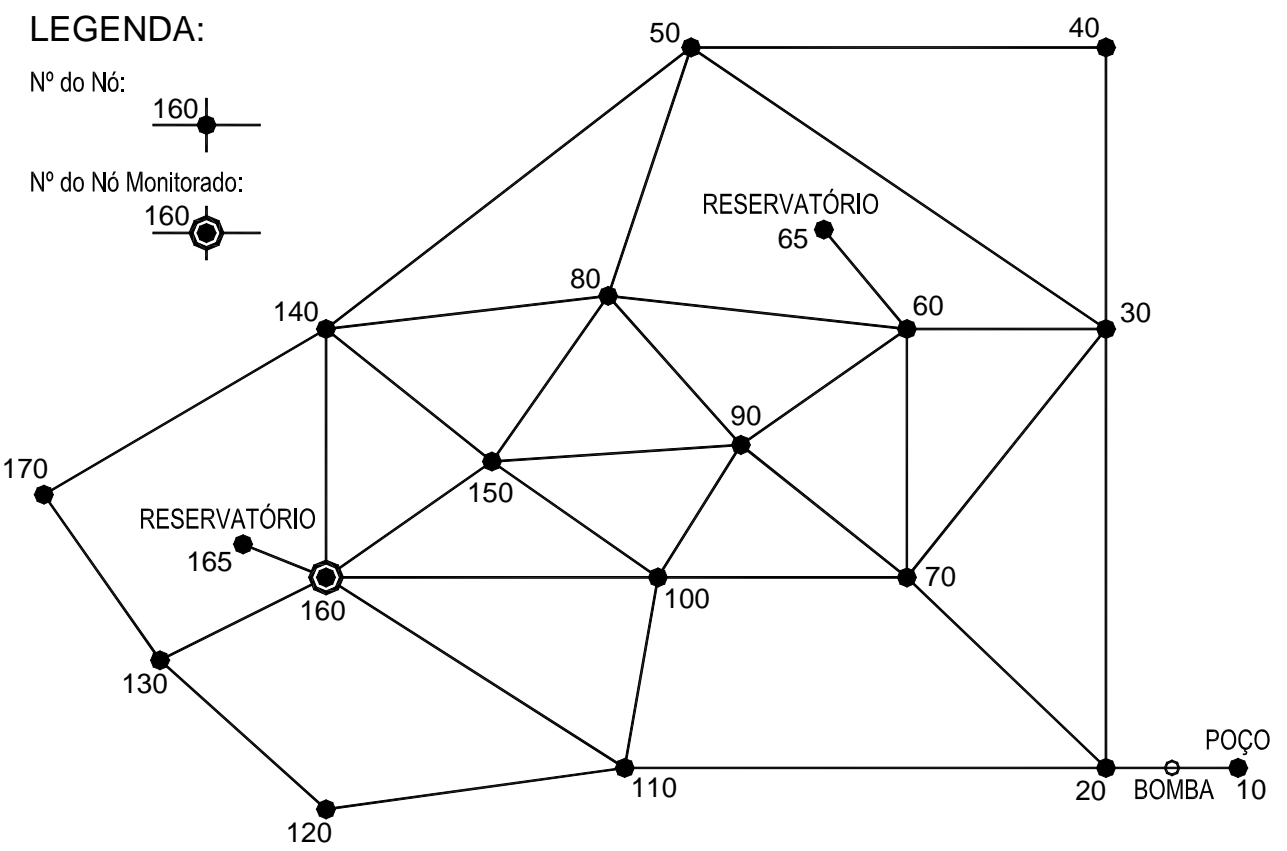

Figura 6.4 - Solução 1 da Rede Anytown para 1 equipamento considerando detecções repetidas.

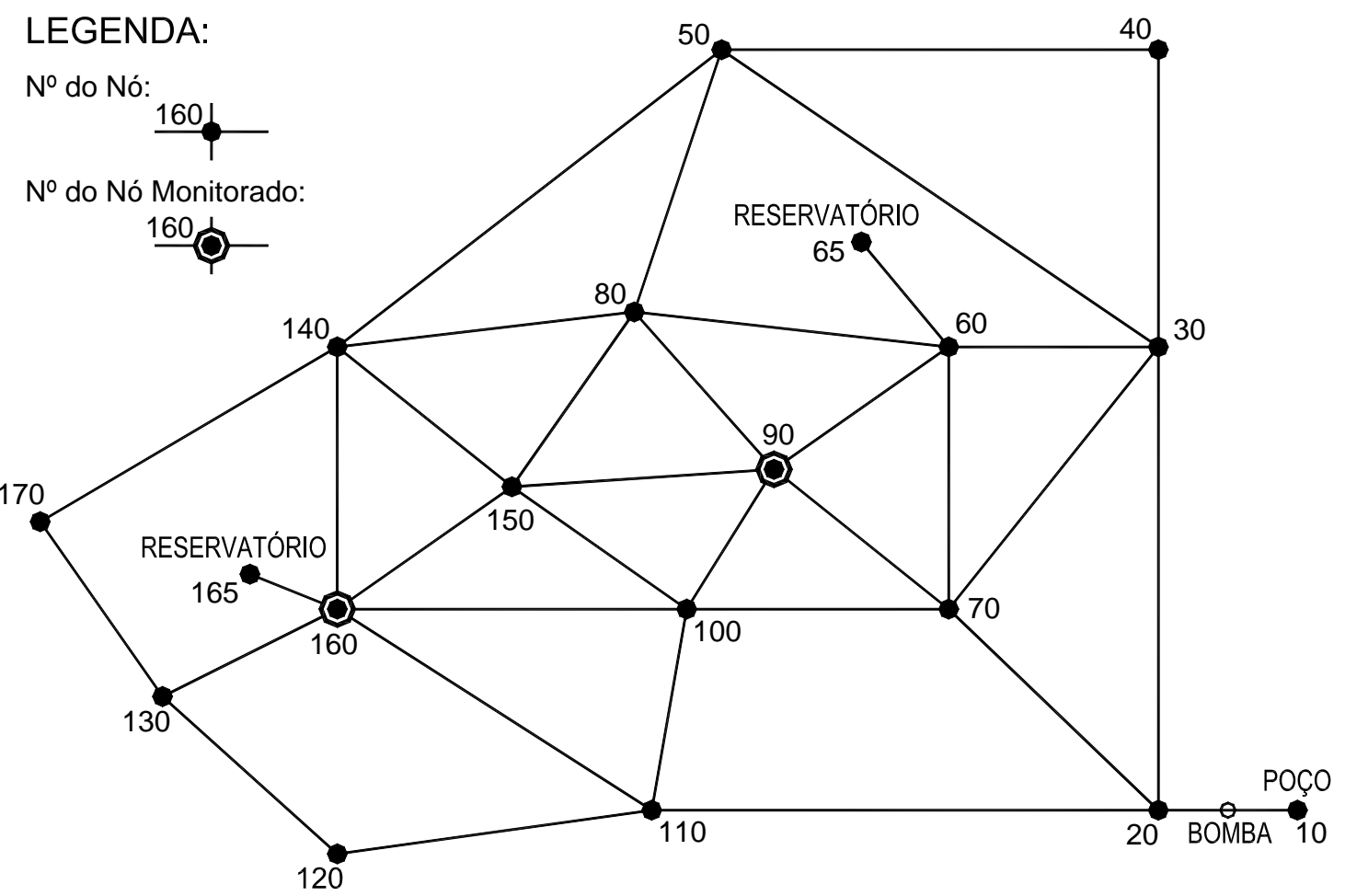

Figura 6.5 - Solução 1 da Rede Anytown para 2 equipamento considerando detecções repetidas. 


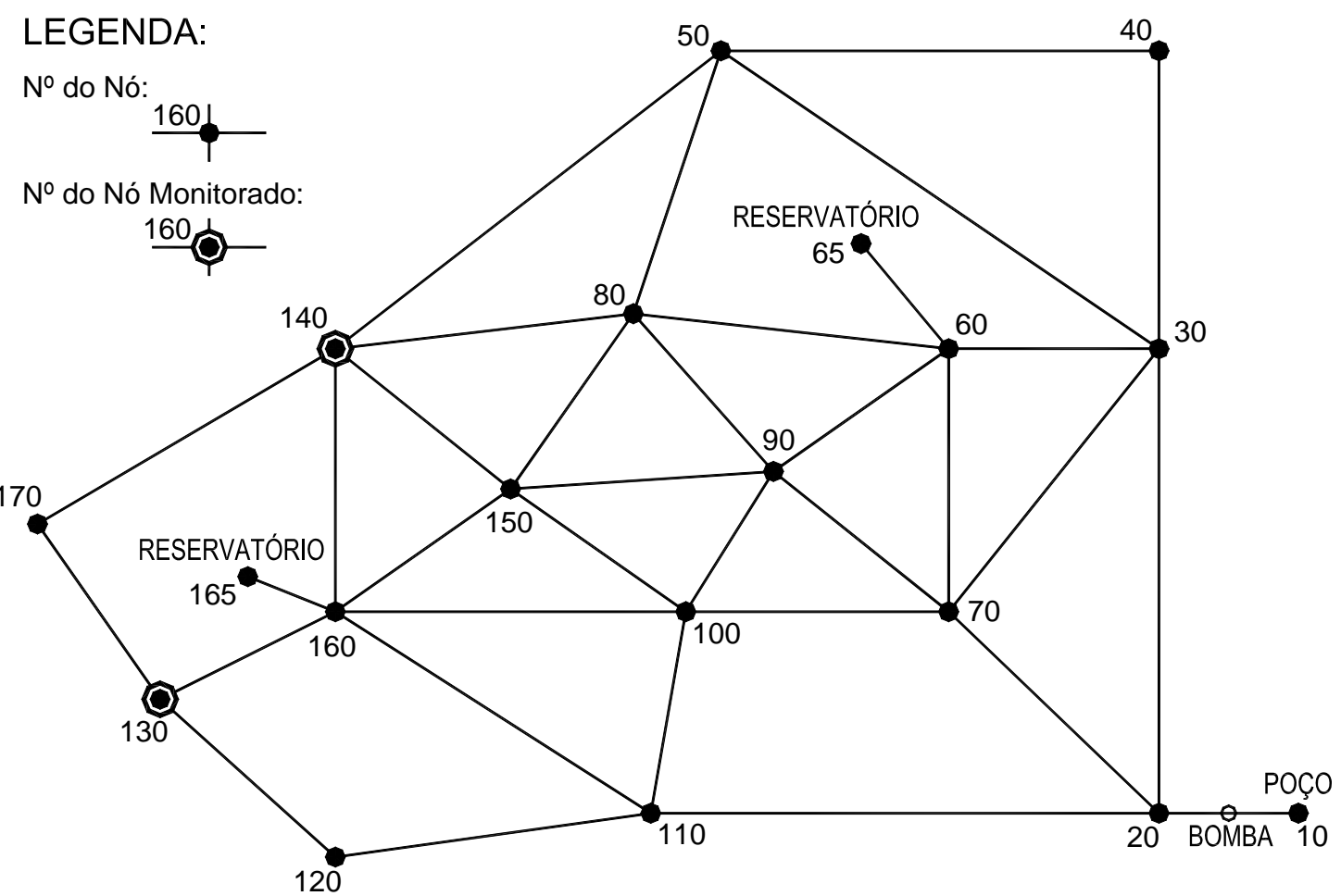

Figura 6.6 - Solução 2 da Rede Anytown para 2 equipamento considerando detecções repetidas.

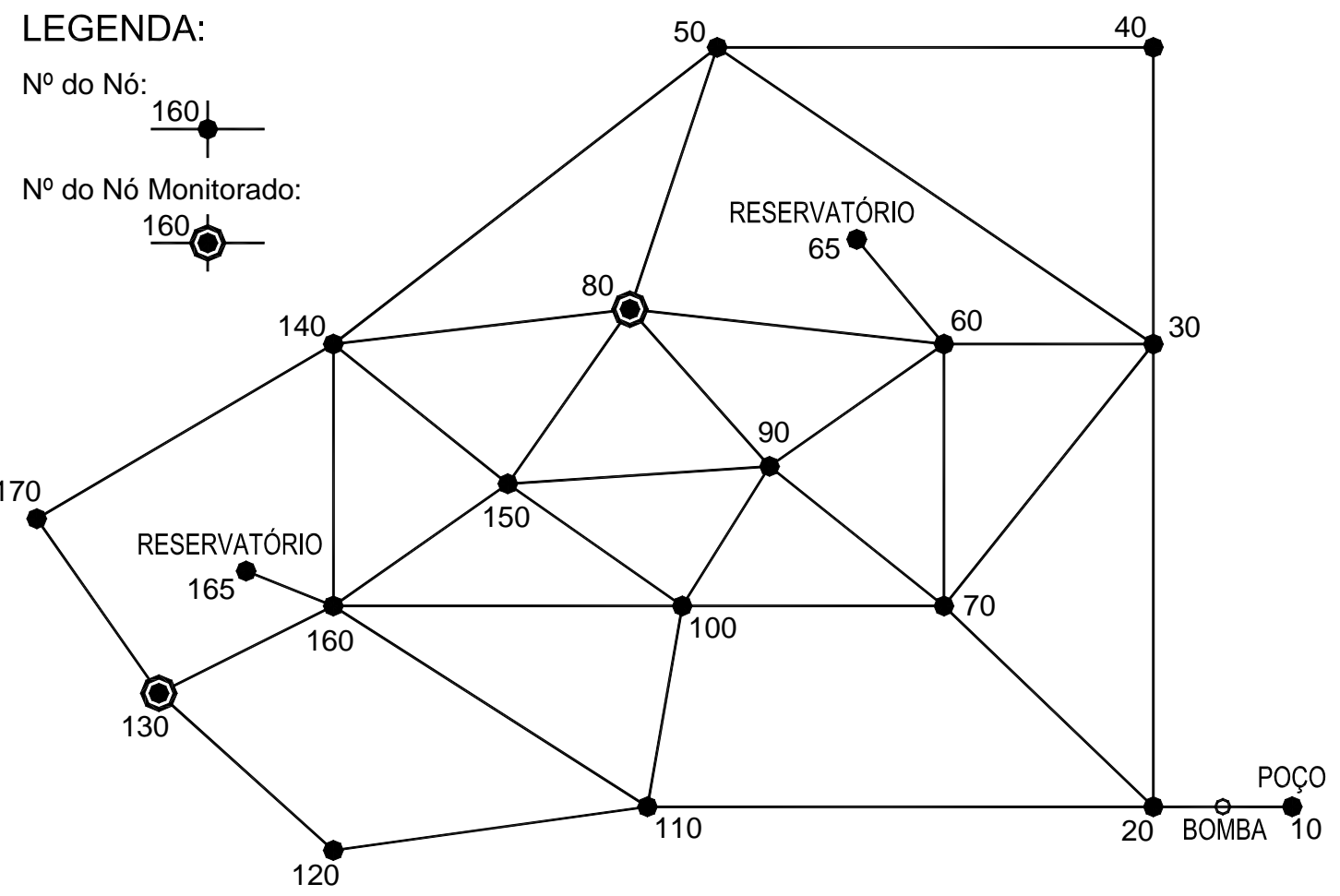

Figura 6.7 - Solução 3 da Rede Anytown para 2 equipamento considerando detecções repetidas. 


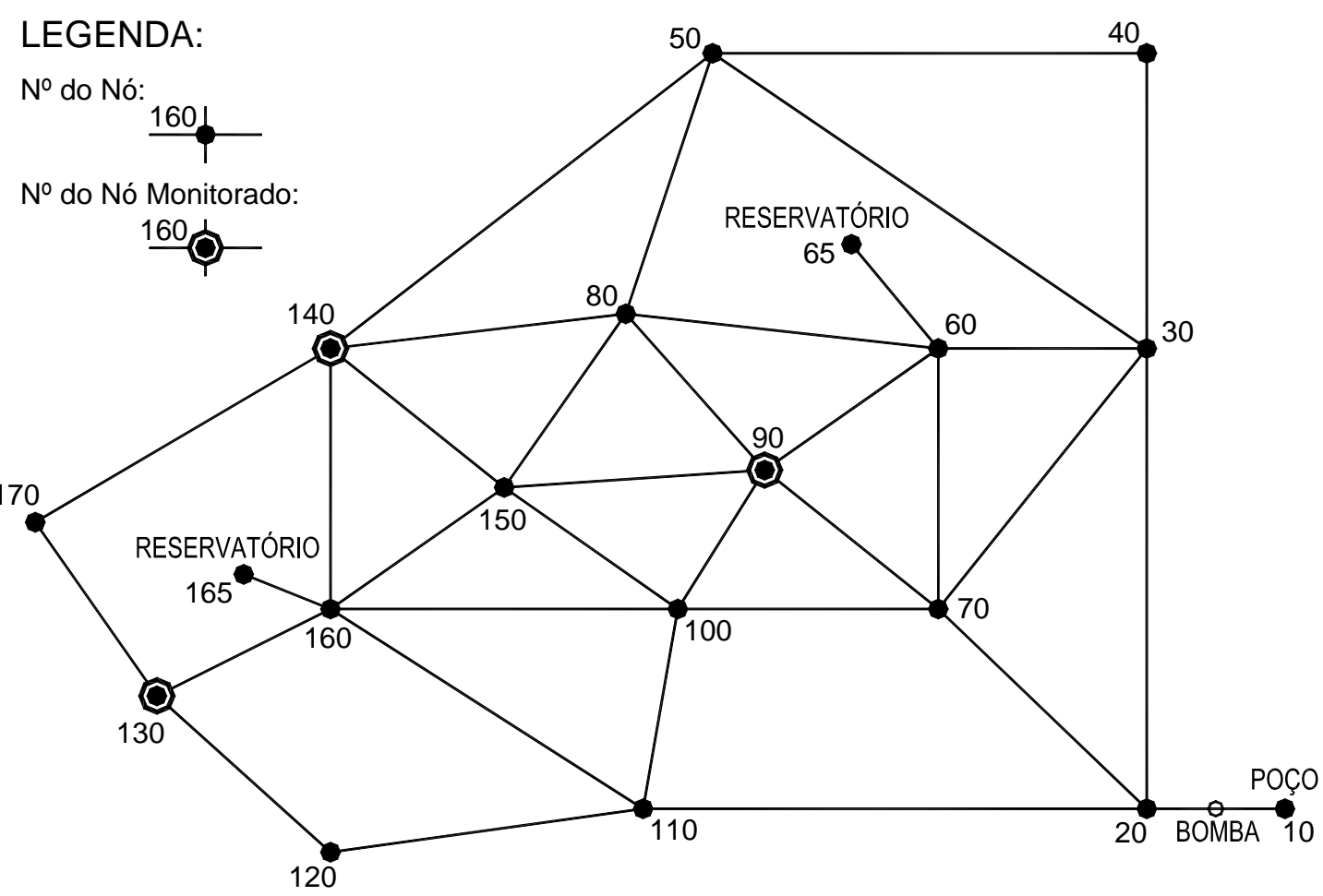

Figura 6.8 - Solução 1 da Rede Anytown para 3 equipamento considerando detecções repetidas.

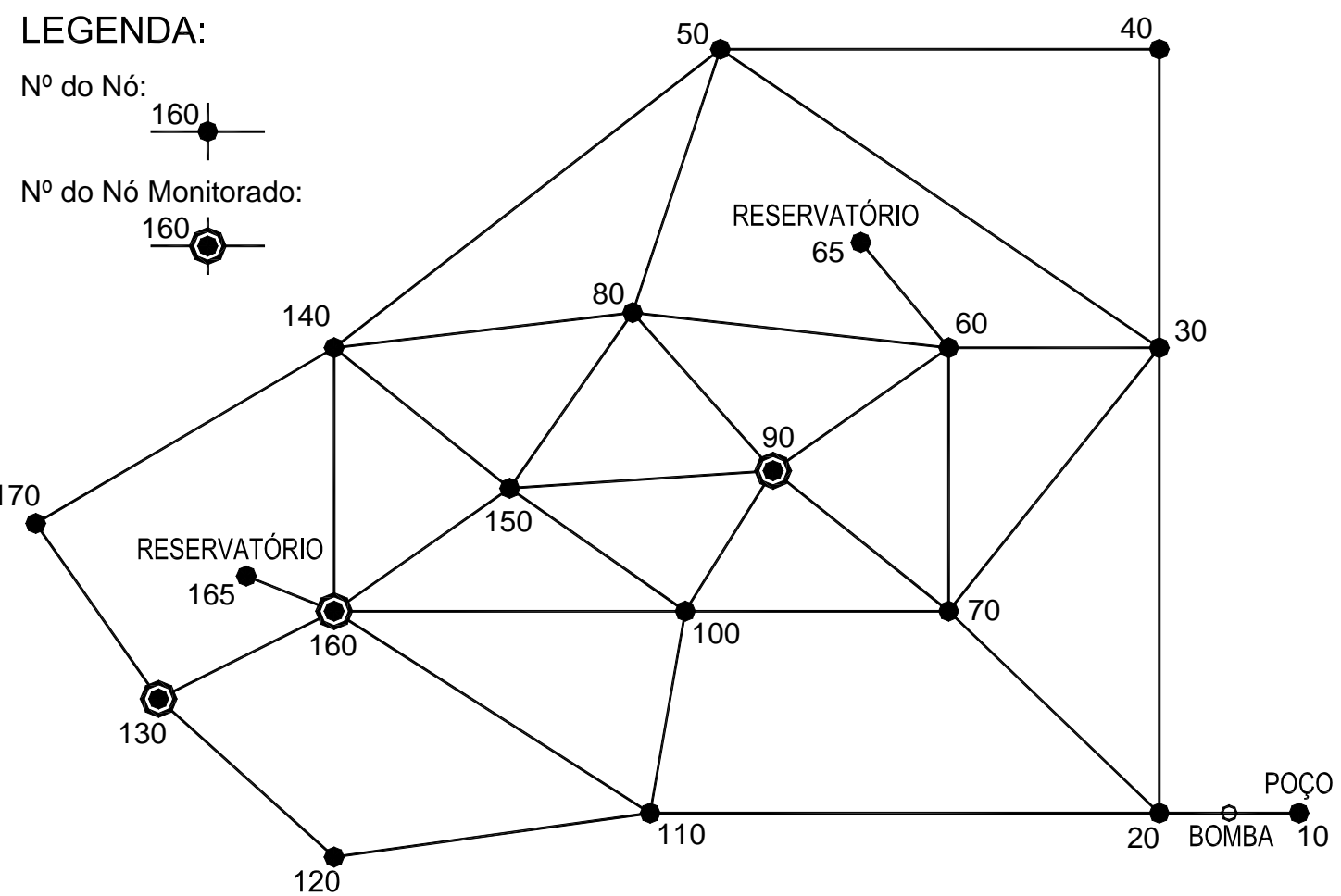

Figura 6.9 - Solução 2 da Rede Anytown para 3 equipamento considerando detecções repetidas. 


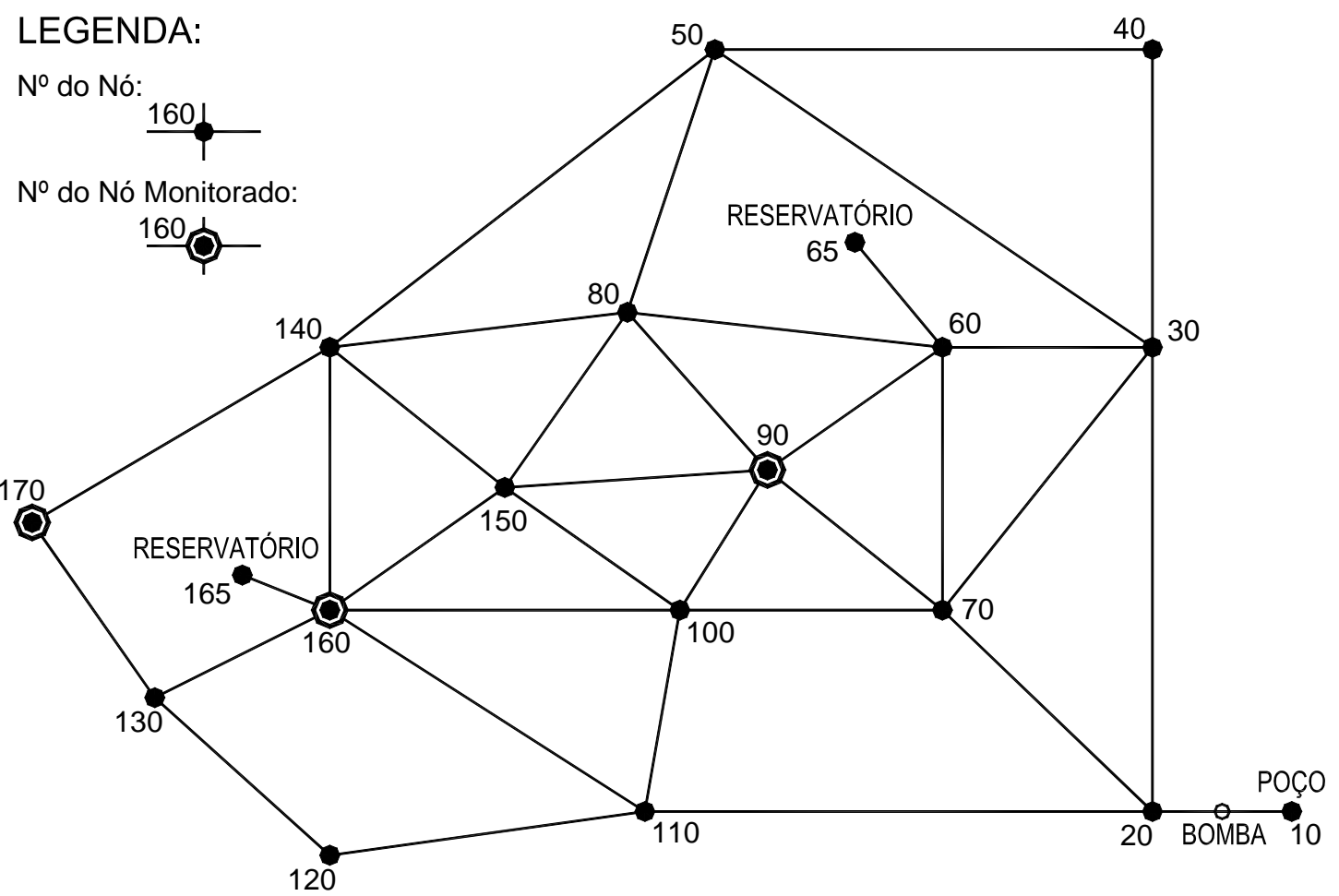

Figura 6.10 - Solução 3 da Rede Anytown para 3 equipamento considerando detecções repetidas.

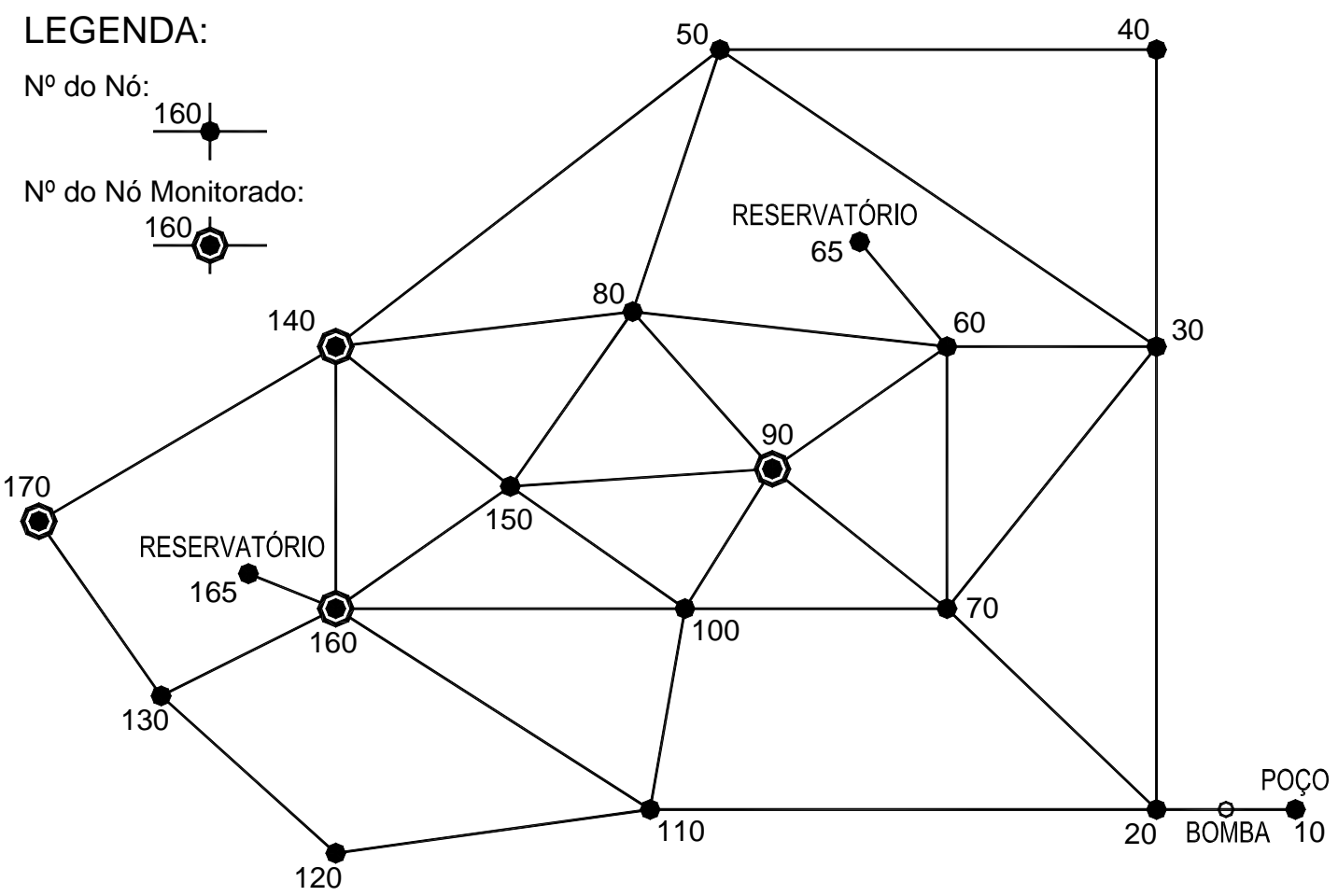

Figura 6.11 - Solução 1 da Rede Anytown para 4 equipamento considerando detecções repetidas. 


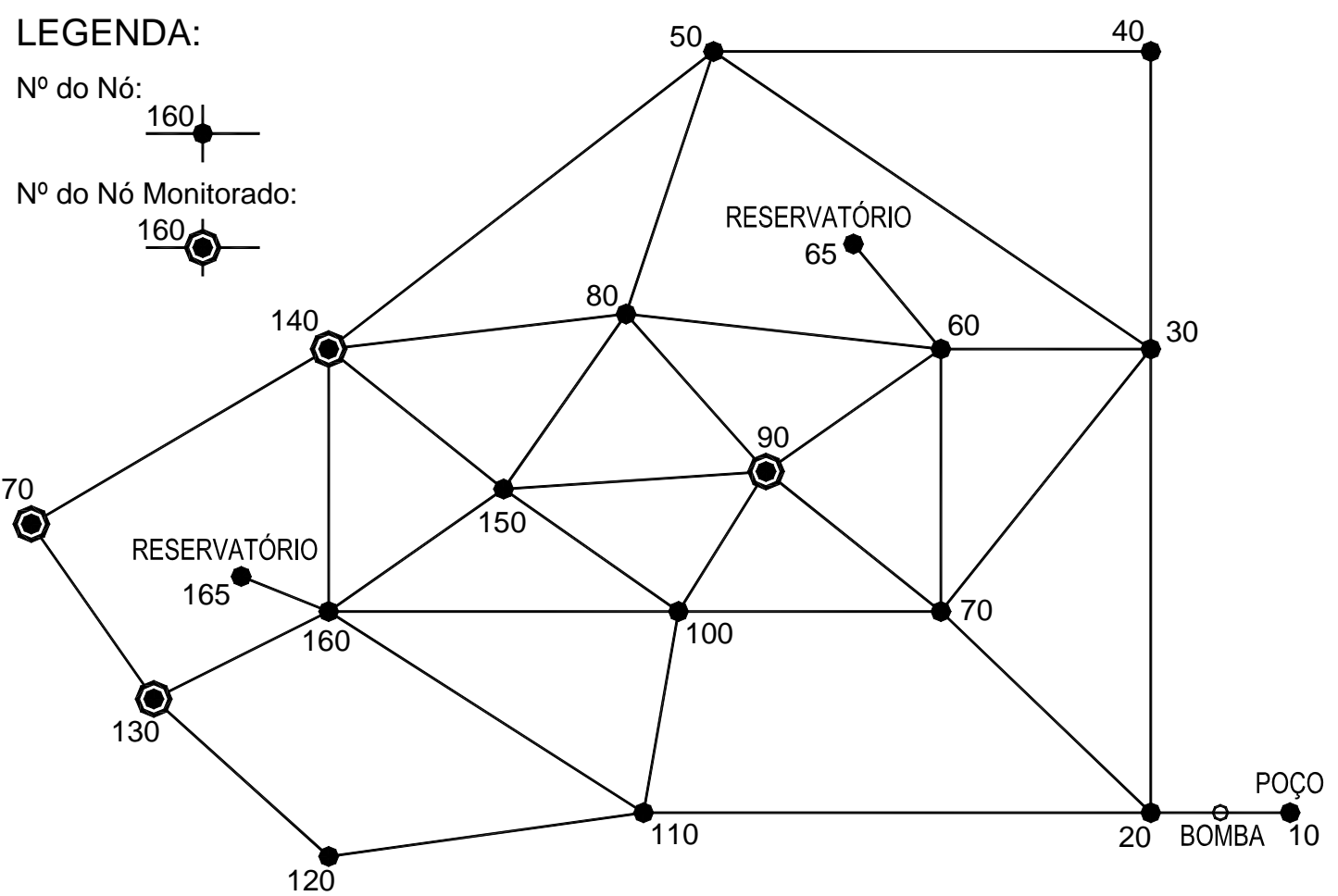

Figura 6.12 - Solução 2 da Rede Anytown para 4 equipamento considerando detecções repetidas.

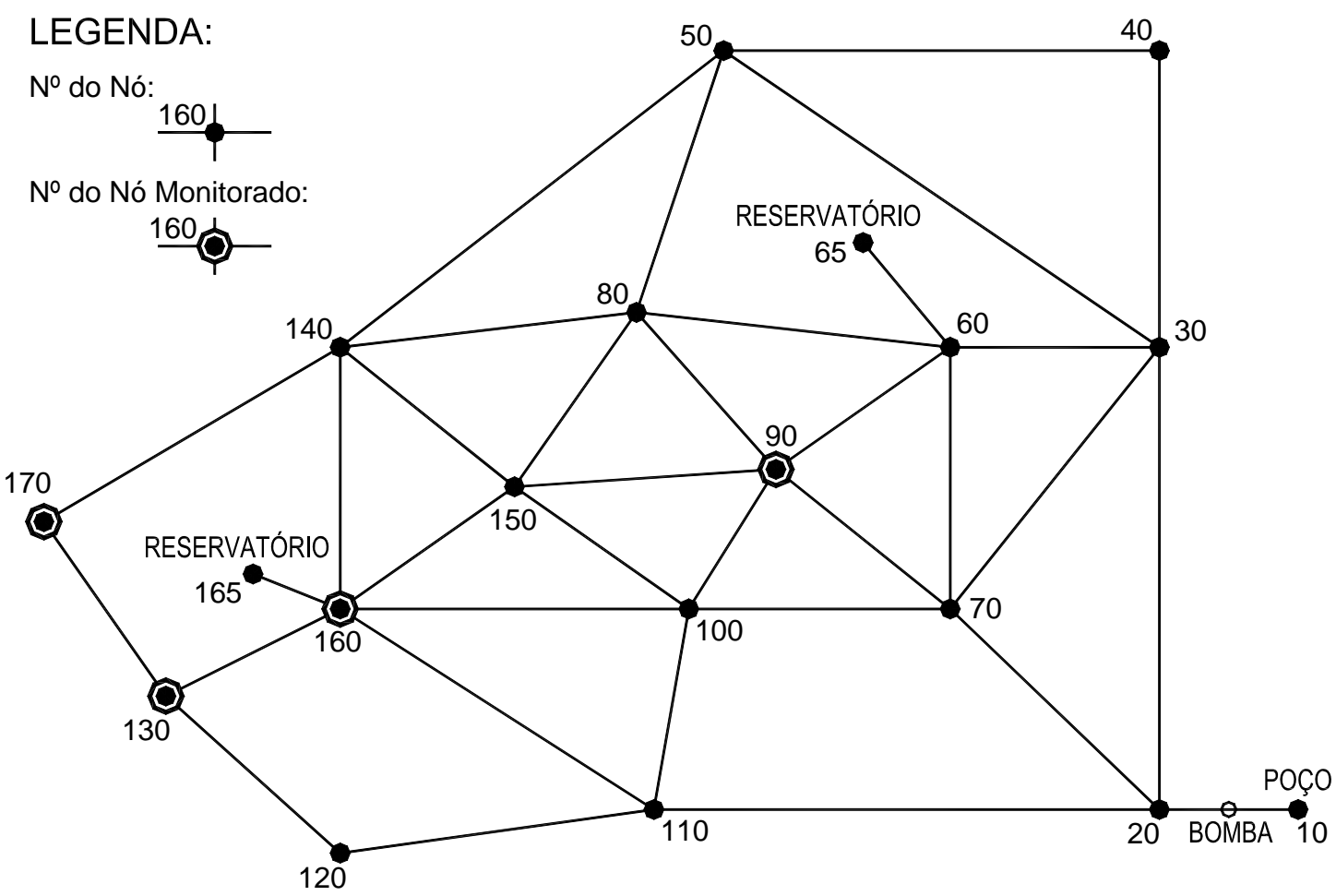

Figura 6.13 - Solução 3 da Rede Anytown para 4 equipamento considerando detecções repetidas. 


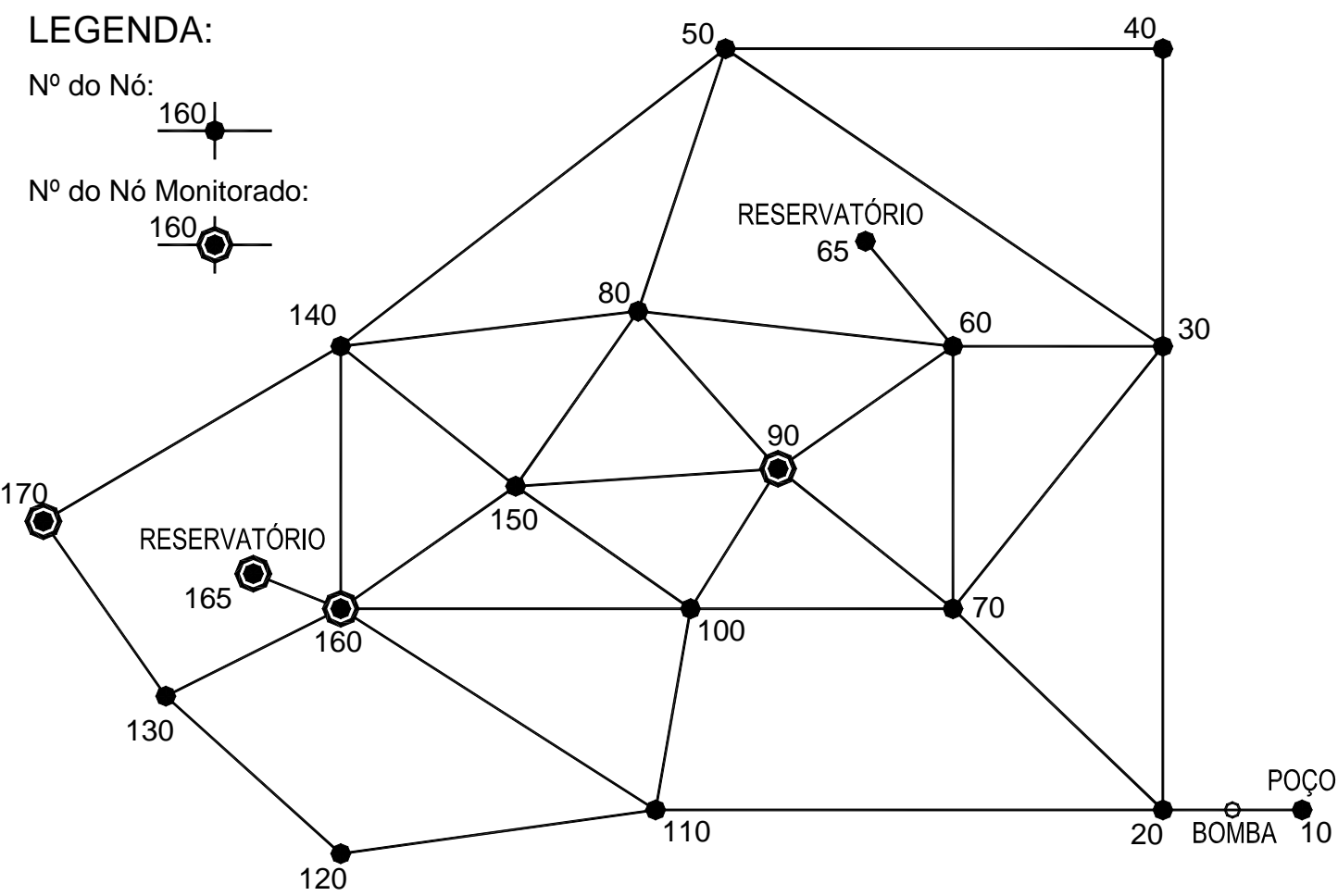

Figura 6.14 - Solução 4 da Rede Anytown para 4 equipamento considerando deteç̧ões repetidas. 


\section{DISCUSSÃO DOS RESULTADOS}

\subsection{Rede Net1}

Os resultados das simulações para a rede Net1, não considerando as detecções repetidas pelas diversas unidades de monitoramento, apresentamse resumidos na Tabela 7.1 e Figura 7.1. Já os resultados das simulações para a rede Net1, considerando as detecções repetidas pelas diversas unidades de monitoramento, apresentam-se resumidos na Tabela 7.2 e Figura 7.2. Tais resultados indicam, para ambas situações:

(1) as mesmas soluções para 1, 2 e 3 unidades foram produzidas, embora os AGs Multiobjetivo tivessem sido rodados a partir de diversas soluções iniciais aleatórias, ou seja, independentemente da população inicial, sempre foram alcançados os mesmos resultados;

(2) a mesma solução de 3 unidades de monitoramento obtida por KESSLER et al. (1998), que considera apenas um único objetivo (volume consumido de água contaminada - VC), foi produzida através do método proposto, já que o objetivo VC é considerado F1;

(3) três unidades de monitoramento são suficientes para a detecção de intrusão intencional de contaminantes e/ou poluentes em todos os 11 nós da rede, antes que os níveis de serviço 1 e 2 sejam ultrapassados, ou seja, o volume consumido de água contaminada pela população e o tempo máximo permitido para a detecção ultrapassam o volume de $283,17 \mathrm{~m}^{3}$ e o tempo de 6 horas respectivamente, até a emissão do sinal de alarme; 
(4) três unidades de monitoramento não foram suficientes para a detecção de intrusão intencional de contaminantes e/ou poluentes em todos os nós antes que o nível de serviço 3 fosse superado, ou seja, a Extensão da Contaminação poderá superar o limite de $15 \%$ do comprimento total da rede previsto.

Tabela 7.1 - Resultados da Rede Net1 não considerando detecções repetidas. $\mathrm{N}^{\mathrm{o}}$ de equipamentos para monitoramento: 01un.

\begin{tabular}{|c|c|c|c|c|}
\hline \hline Solução & \multicolumn{3}{|c|}{ no espaço de objetivos } & no espaço de decisão \\
\hline $\mathrm{N}^{\mathrm{o}}$. & $\mathrm{F} 1$ & $\mathrm{~F} 2$ & $\mathrm{~F} 3$ & Nó de monitoramento \\
\hline 1 & $7^{*}$ & $7^{*}$ & $4^{*}$ & 22 \\
\hline \hline
\end{tabular}

$\mathrm{N}^{\mathrm{o}}$ de equipamentos para monitoramento: $02 \mathrm{un}$.

\begin{tabular}{|c|c|c|c|c|}
\hline \hline Solução & \multicolumn{3}{|c|}{ no espaço de objetivos } & no espaço de decisão \\
\hline $\mathrm{N}^{\mathrm{o}}$. & $\mathrm{F} 1$ & $\mathrm{~F} 2$ & $\mathrm{~F} 3$ & Nós de monitoramento \\
\hline 1 & $9^{*}$ & 9 & $6^{*}$ & 23 e 22 \\
\hline 2 & 6 & $10^{*}$ & 6 & 13 e 32 \\
\hline \hline
\end{tabular}

$\mathrm{N}^{\mathrm{o}}$ de equipamentos para monitoramento: 03un.

\begin{tabular}{|c|c|c|c|c||}
\hline \hline Solução & \multicolumn{3}{|c||}{ no espaço de objetivos } & no espaço de decisão \\
\hline $\mathrm{N}^{\mathrm{o}}$. & $\mathrm{F} 1$ & $\mathrm{~F} 2$ & $\mathrm{~F} 3$ & Nós de monitoramento \\
\hline 1 & $11^{*}$ & $11^{*}$ & $8^{*}$ & 23,32 e 22 ** \\
\hline \hline
\end{tabular}

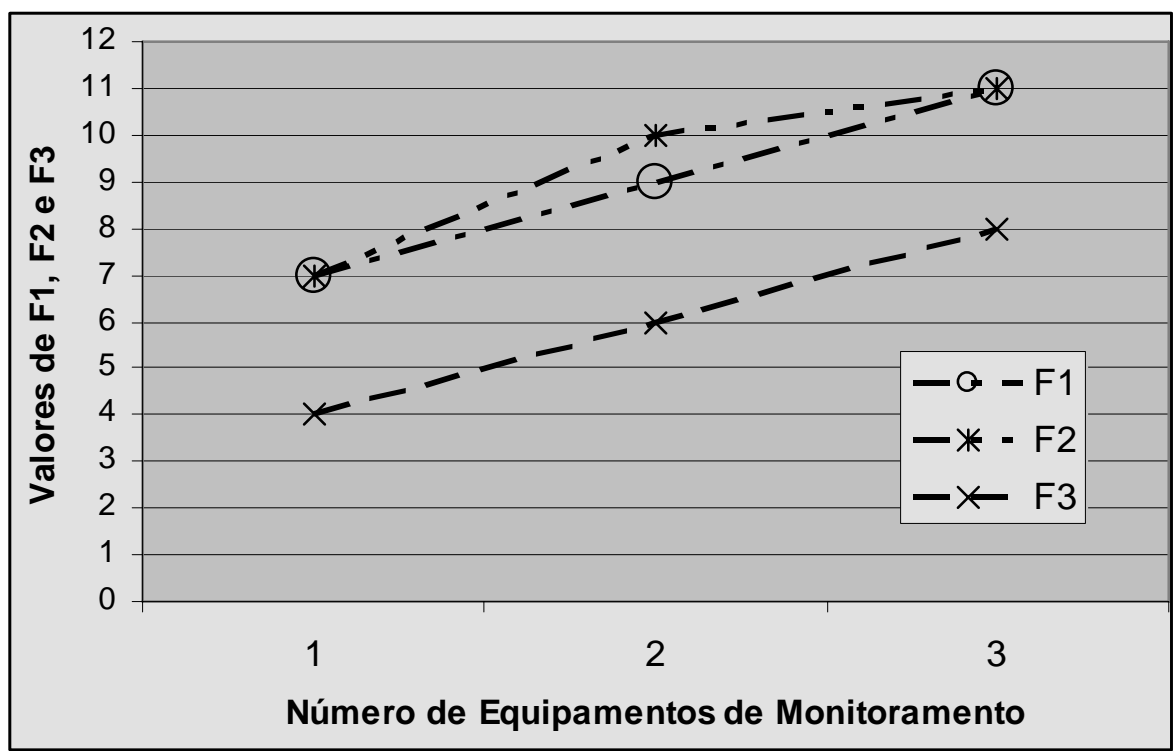

Figura 7.1 - Gráfico Valores máximos de F1, F2 e F3 x Número de Equipamentos para Rede Net1 não considerando detecções repetidas. 
Tabela 7.2 - Resultados da Rede Net1 considerando detecções repetidas. $\mathrm{N}^{\mathrm{o}}$ de equipamentos para monitoramento: $01 \mathrm{un}$.

\begin{tabular}{|c|c|c|c|c|}
\hline Solução & \multicolumn{3}{|c|}{ no espaço de objetivos } & no espaço de decisão \\
\hline $\mathrm{N}^{\mathrm{o}}$. & $\mathrm{F} 1$ & $\mathrm{~F} 2$ & $\mathrm{~F} 3$ & Nó de monitoramento \\
\hline 1 & 7 & 7 & 4 & 22 \\
\hline \multicolumn{5}{|c|}{$\mathrm{N}^{0}$ de equipamentos para monitoramento: $02 \mathrm{un}$. } \\
\hline Solução & \multicolumn{3}{|c|}{ no espaço de objetivos } & no espaço de decisão \\
\hline $\mathrm{N}^{\mathrm{o}}$. & F1 & $\mathrm{F} 2$ & F3 & Nós de monitoramento \\
\hline 1 & 6.02 & 10.03 & 6 & 13 e 32 \\
\hline 2 & 9.03 & 9.05 & 6.01 & 32 e 22 \\
\hline \multicolumn{5}{|c|}{$\mathrm{N}^{\circ}$ de equipamentos para monitoramento: 03un. } \\
\hline Solução & \multicolumn{3}{|c|}{ no espaço de objetivos } & no espaço de decisão \\
\hline $\mathrm{N}^{\mathrm{o}}$. & $\mathrm{F} 1$ & $\mathrm{~F} 2$ & $\mathrm{~F} 3$ & Nós de monitoramento \\
\hline 1 & 11.03 & 11.06 & 8.02 & 22,23 e $32 * *$ \\
\hline
\end{tabular}

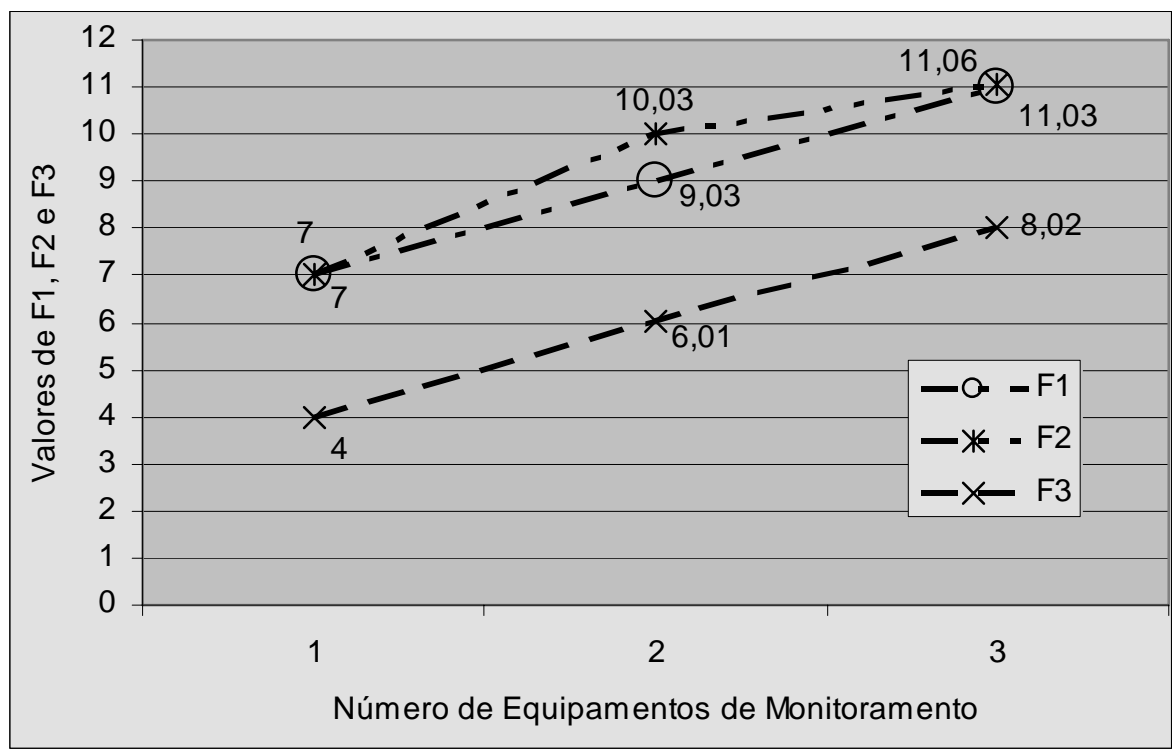

Figura 7.2 - Gráfico Valores máximos de F1, F2 e F3 x Número de Equipamentos para Rede Net1 considerando detecções repetidas. 


\subsection{Rede Anytown}

Os resultados das simulações para a Rede Anytown, não considerando as detecções repetidas pelas diversas unidades de monitoramento, apresentam-se resumidos na Tabela 7.3 e Figura 7.3 para o nível de serviço 1 de $468,10 \mathrm{~m}^{3}$, e na Tabela 7.5 e Figura 7.5 para o nível de serviço 1 de $1245,15 \mathrm{~m}^{3}$. Já os resultados das simulações para a Rede Anytown considerando acréscimo por detecções repetidas pelas diversas unidades de monitoramento apresentamse resumidos na Tabela 7.4 e Figura 7.4 para o nível de serviço 1 de $468,10 \mathrm{~m}^{3}$, e na Tabela 7.6 e Figura 7.6 para o nível de serviço 1 de $1245,15 \mathrm{~m}^{3}$. Tais resultados indicam:

(1) as mesmas soluções de 1, 2, 3 e 4 unidades foram produzidas, embora os AGs Multiobjetivo tivessem sido rodados a partir de diversas soluções iniciais aleatórias, ou seja, independentemente da população inicial, sempre foram alcançados os mesmos resultados;

(2) a mesma solução de 4 unidades de monitoramento obtida por KESSLER et al. (1998), que considera apenas um único objetivo (volume consumido de água contaminada - VC), foi produzida através do método proposto, já que o objetivo VC é considerado F1;

(3) para o nível $\mathrm{VC}=1245,15 \mathrm{~m}^{3}$, três unidades de monitoramento são suficientes para a detecção de intrusão intencional de contaminantes e/ou poluentes em todos os 19 nós da rede, antes que o nível de serviço 1 seja ultrapassado. Para KESSLER et al (1998) o nível de serviço 1 só é atingido com 4 unidades de monitoramento; 
(4) Uma unidade de monitoramento já foi suficiente para a detecção de intrusão intencional de contaminantes e/ou poluentes em todos os nós antes que o nível de serviço 3 fosse superado.

(5) A solução que contém o ponto 165 (Tanque) na Tabela 7.6 foi produzida devido à proximidade entre esse nó e o nó 160 , tendo os dois em comum a detecção praticamente dos mesmos nós fontes de poluição. Sendo assim, estes pontos aumentam o número de detecções repetidas.

Tabela 7.3 - Resultados da Rede Anytown não considerando detecções repetidas: Nível VC $=468,10 \mathrm{~m}^{3}$.

\begin{tabular}{|c|c|c|c|c|}
\hline \multicolumn{5}{|c|}{$\mathrm{N}^{\circ}$ de equipamentos para monitoramento: 01un. } \\
\hline Solução & \multicolumn{3}{|c|}{ no espaço de objetivos } & no espaço de decisão \\
\hline $\mathrm{N}^{\mathrm{o}}$. & $\mathrm{F} 1$ & $\mathrm{~F} 2$ & F3 & Nós de monitoramento \\
\hline 1 & 6 & $13 *$ & $19 *$ & 160 \\
\hline 2 & $7 *$ & 9 & 19 & 90 \\
\hline \multicolumn{5}{|c|}{ No de equipamentos para monitoramento: $02 \mathrm{un}$. } \\
\hline Solução & \multicolumn{3}{|c|}{ no espaço de objetivos } & no espaço de decisão \\
\hline $\mathrm{N}^{\mathrm{o}}$. & $\mathrm{F} 1$ & $\mathrm{~F} 2$ & F3 & Nós de monitoramento \\
\hline 1 & $13 *$ & 15 & $19 *$ & 160 e 90 \\
\hline 2 & 10 & $17 *$ & 19 & 130 e 80 \\
\hline \multicolumn{5}{|c|}{$\mathrm{N}^{\circ}$ de equipamentos para monitoramento: 03un. } \\
\hline Solução & \multicolumn{3}{|c|}{ no espaço de objetivos } & no espaço de decisão \\
\hline $\mathrm{N}^{\mathrm{o}}$. & $\mathrm{F} 1$ & $\mathrm{~F} 2$ & $\mathrm{~F} 3$ & Nós de monitoramento \\
\hline 1 & 15 & $18^{*}$ & $19 *$ & 140,90 e 130 \\
\hline 2 & $16^{*}$ & 17 & 19 & 90,160 e 170 \\
\hline \multicolumn{5}{|c|}{ No de equipamentos para monitoramento: 04un. } \\
\hline Solução & \multicolumn{3}{|c|}{ no espaço de objetivos } & no espaço de decisão \\
\hline $\mathrm{N}^{\mathrm{o}}$. & $\mathrm{F} 1$ & $\mathrm{~F} 2$ & F3 & Nós de monitoramento \\
\hline 1 & 16 & $19 *$ & $19 *$ & $130,170,90$ e 80 \\
\hline 2 & $19 *$ & 18 & 19 & $90,160,170$ e $80 * *$ \\
\hline \multicolumn{5}{|c|}{$\begin{array}{l}\text { Obs.: * valores máximos de F1, F2 e F3 utilizados nos gráficos } \\
\quad * * \text { solução apontada por KESSLER et al. (1998) } \\
\end{array}$} \\
\hline
\end{tabular}




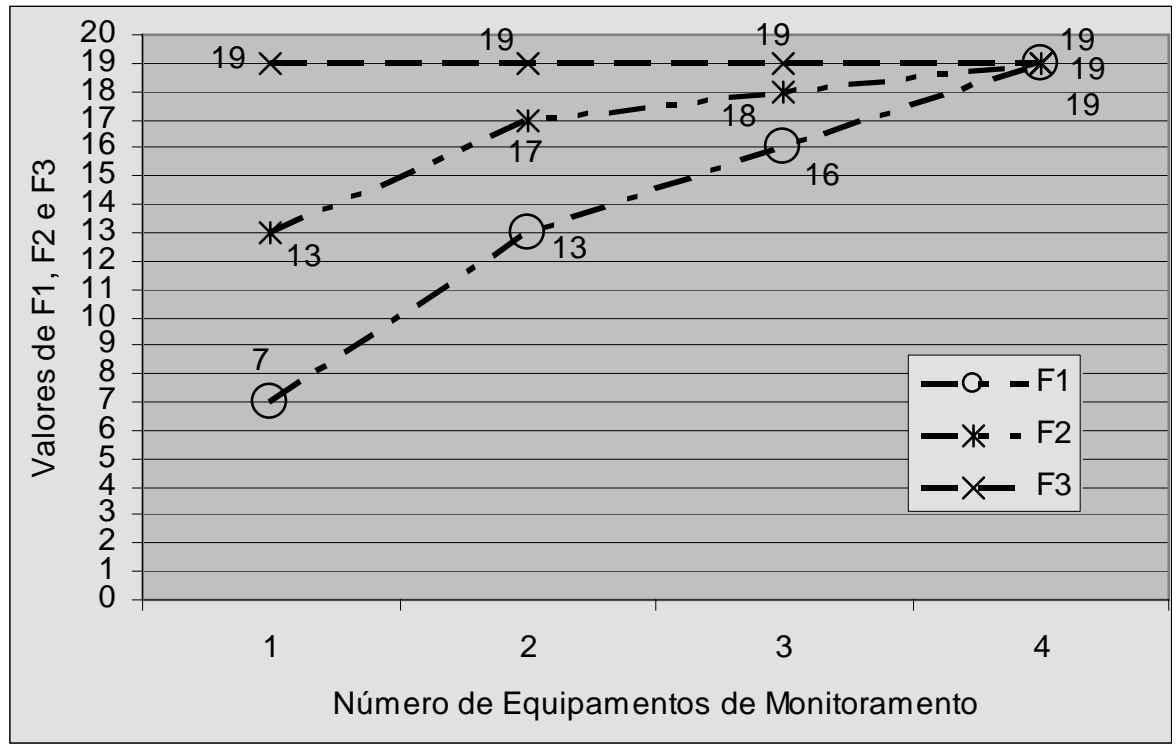

Figura 7.3 - Gráfico Valores máximos de F1, F2 e F3 x Número de Equipamentos para

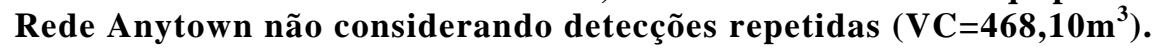

Tabela 7.4 - Resultados da Rede Anytown considerando detecções repetidas: Nível VC $=468,10 \mathrm{~m}^{3}$.

$\mathrm{N}^{0}$ de equipamentos para monitoramento: $01 \mathrm{un}$.

\begin{tabular}{|c|c|c|c|c|}
\hline \hline Solução & \multicolumn{3}{|c|}{ no espaço de objetivos } & no espaço de decisão \\
\hline$N^{\text {o }}$ & F1 & F2 & F3 & Nó de monitoramento \\
\hline 1 & $7^{*}$ & 9 & $19^{*}$ & 90 \\
\hline 2 & 6 & $13^{*}$ & 19 & 160 \\
\hline \hline
\end{tabular}

$\mathrm{N}^{\mathrm{o}}$ de equipamentos para monitoramento: $02 \mathrm{un}$.

\begin{tabular}{||c|c|c|c|c|}
\hline \hline Solução & \multicolumn{3}{|c|}{ no espaço de objetivos } & no espaço de decisão \\
\hline $\mathrm{N}^{\mathrm{o}}$. & $\mathrm{F} 1$ & $\mathrm{~F} 2$ & $\mathrm{~F} 3$ & Nós de monitoramento \\
\hline 1 & $13^{*}$ & 15.07 & $19.19^{*}$ & 90,160 \\
\hline 2 & 8 & $17.06^{*}$ & 19.19 & 130,140 \\
\hline 3 & 10 & 17.03 & 19.19 & 80,130 \\
\hline \hline
\end{tabular}

$\mathrm{N}^{\mathrm{o}}$ de equipamentos para monitoramento: 03un.

\begin{tabular}{||c|c|c|c|c||}
\hline \hline Solução & \multicolumn{3}{|c|}{ no espaço de objetivos } & no espaço de decisão \\
\hline $\mathrm{N}^{\mathrm{o}}$. & $\mathrm{F} 1$ & $\mathrm{~F} 2$ & $\mathrm{~F} 3$ & Nós de monitoramento \\
\hline 1 & 15 & $18.14^{*}$ & $19.38^{*}$ & $130,90,140$ \\
\hline 2 & $16.03^{*}$ & 16.15 & 19.38 & $160,80,90$ \\
\hline 3 & 16.01 & 17.09 & 19.38 & $160,90,170$ \\
\hline 4 & 15.02 & 17.16 & 19.38 & $160,90,130$ \\
\hline
\end{tabular}




\begin{tabular}{|c|c|c|c|c|}
\hline \multicolumn{5}{|c|}{$\mathrm{N}^{0}$ de equipamentos para monitoramento: 04un. } \\
\hline Solução & \multicolumn{3}{|c|}{ no espaço de objetivos } & no espaço de decisão \\
\hline $\mathrm{N}^{\mathrm{o}}$. & F1 & F2 & F3 & Nós de monitoramento \\
\hline 1 & 16.05 & 19.14 & $19.57^{*}$ & $130,80,90,170$ \\
\hline 2 & 17.04 & 18.27 & 19.57 & $130,90,140,160$ \\
\hline 3 & 18.05 & 18.24 & 19.57 & $130,80,90,160$ \\
\hline 4 & 16.03 & $19.17 *$ & 19.57 & $130,90,140,170$ \\
\hline 5 & $19,04 *$ & 18.17 & 19.57 & $160,80,90,170 * *$ \\
\hline \multicolumn{5}{|c|}{$\begin{array}{l}\text { Obs.: * valores máximos de F1, F2 e F3 utilizados nos gráficos } \\
\text { **solução apontada por KESSLER et al. (1998) }\end{array}$} \\
\hline
\end{tabular}

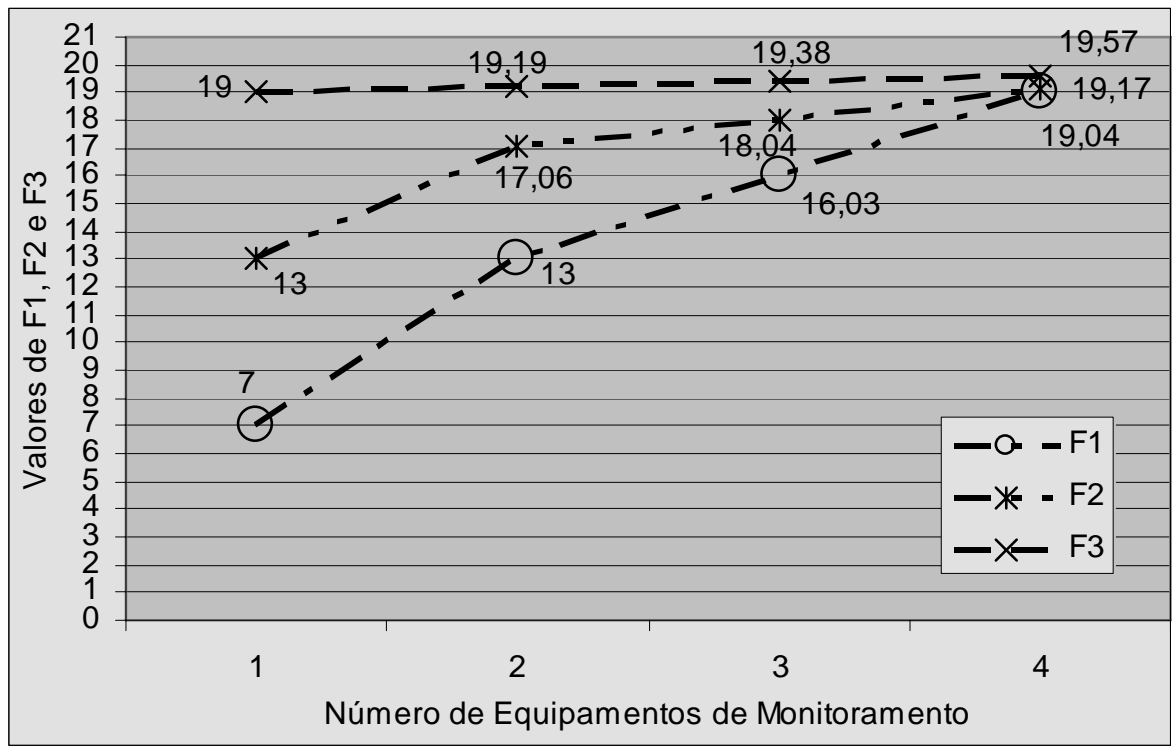

Figura 7.4 - Gráfico Valores máximos de F1, F2 e F3 x Número de Equipamentos para Rede Anytown considerando deteç̧ões repetidas $\left(\mathrm{VC}=468,10 \mathrm{~m}^{3}\right)$.

Tabela 7.5 - Resultados da Rede Anytown não considerando deteç̧ões repetidas: Nível VC $=1245,15 \mathrm{~m}^{3}$.

\begin{tabular}{|c|c|c|c|c|}
\hline \multicolumn{5}{|c|}{$\mathrm{N}^{\circ}$ de equipamentos para monitoramento: 01un. } \\
\hline Solução & \multicolumn{3}{|c|}{ no espaço de objetivos } & no espaço de decisão \\
\hline $\mathrm{N}^{\mathrm{o}}$. & F1 & $\mathrm{F} 2$ & F3 & Nó de monitoramento \\
\hline 1 & $12^{*}$ & $13^{*}$ & $19^{*}$ & 160 \\
\hline \multicolumn{5}{|c|}{ No de equipamentos para monitoramento: $02 \mathrm{un}$. } \\
\hline Solução & \multicolumn{3}{|c|}{ no espaço de objetivos } & no espaço de decisão \\
\hline $\mathrm{N}^{\mathrm{o}}$. & F1 & $\mathrm{F} 2$ & F3 & Nós de monitoramento \\
\hline 1 & 15 & $17^{*}$ & $19 *$ & 80,130 \\
\hline 2 & $16^{*}$ & 15 & 19 & 90,160 \\
\hline
\end{tabular}




\begin{tabular}{|c|c|c|c|c|}
\hline \multicolumn{5}{|c|}{$\mathrm{N}^{o}$ de equipamentos para monitoramento: $03 \mathrm{un}$. } \\
\hline Solução & \multicolumn{3}{|c|}{ no espaço de objetivos } & no espaço de decisão \\
\hline $\mathrm{N}^{\mathrm{o}}$. & $\mathrm{F} 1$ & $\mathrm{~F} 2$ & F3 & Nós de monitoramento \\
\hline 1 & $19^{*}$ & 17 & $19^{*}$ & $90,160,170$ \\
\hline 2 & 18 & $18^{*}$ & 19 & $80,130,90$ \\
\hline \multicolumn{5}{|c|}{ No de equipamentos para monitoramento: 04un. } \\
\hline Solução & \multicolumn{3}{|c|}{ no espaço de objetivos } & no espaço de decisão \\
\hline $\mathrm{N}^{\mathrm{o}}$. & $\mathrm{F} 1$ & $\mathrm{~F} 2$ & F3 & Nós de monitoramento \\
\hline 1 & $19^{*}$ & $19 *$ & $19^{*}$ & $170,130,80,90$ \\
\hline
\end{tabular}

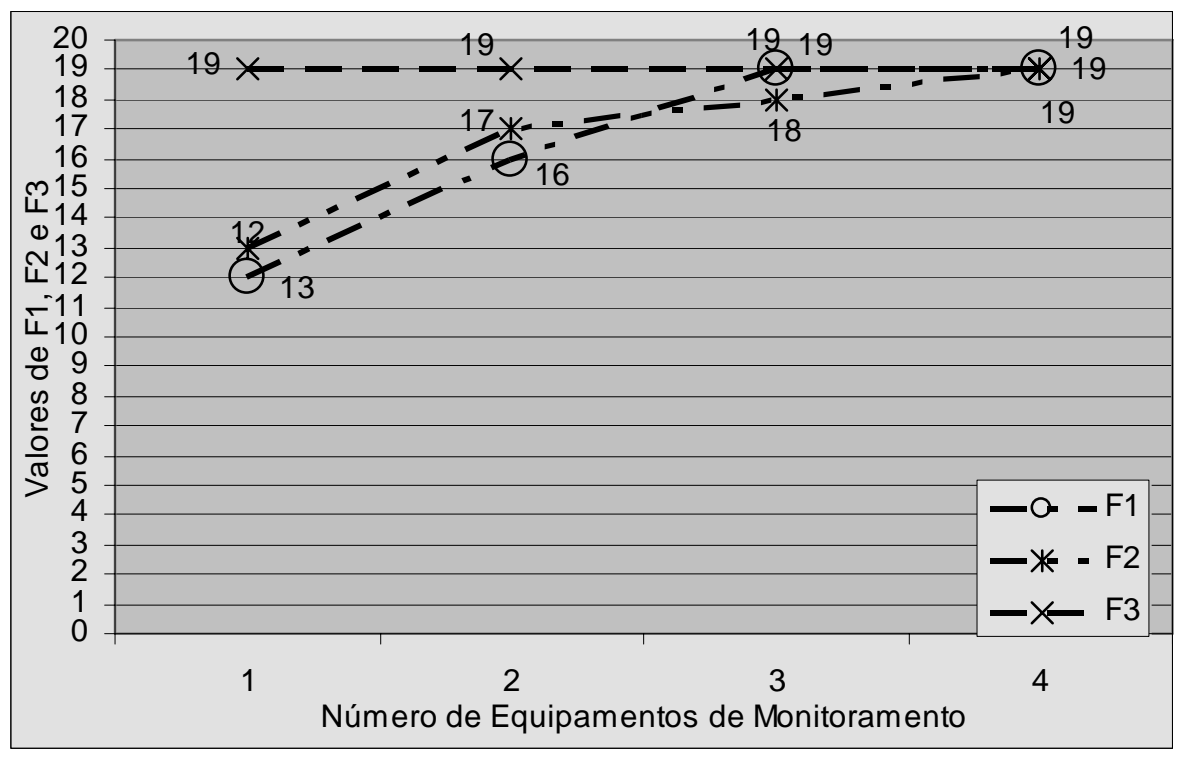

Figura 7.5 - Gráfico Valores máximos de F1, F2 e F3 x Número de Equipamentos para Rede Anytown não considerando detecções repetidas (VC=1245,15 $\mathrm{m}^{3}$ ).

Tabela 7.6 - Resultados da Rede Anytown considerando detecções repetidas: Nível VC $=1245,15 \mathrm{~m}^{3}$.

$\mathrm{N}^{\mathrm{o}}$ de equipamentos para monitoramento: $01 \mathrm{un}$.

\begin{tabular}{||c|c|c|c|c|}
\hline \hline Solução & \multicolumn{3}{|c|}{ no espaço de objetivos } & no espaço de decisão \\
\hline $\mathrm{N}^{\mathrm{o}}$. & $\mathrm{F} 1$ & $\mathrm{~F} 2$ & $\mathrm{~F} 3$ & Nós de monitoramento \\
\hline 1 & $12^{*}$ & $13^{*}$ & $19^{*}$ & 160 \\
\hline \hline
\end{tabular}

$\mathrm{N}^{\mathrm{o}}$ de equipamentos para monitoramento: $02 \mathrm{un}$.

\begin{tabular}{||c|c|c|c|c||}
\hline \hline \multirow{2}{*}{ Solução } & \multicolumn{3}{|c|}{ no espaço de objetivos } & no espaço de decisão \\
\hline $\mathrm{N}^{\mathrm{o}}$. & $\mathrm{F} 1$ & $\mathrm{~F} 2$ & $\mathrm{~F} 3$ & Nós de monitoramento \\
\hline 1 & $16.04^{*}$ & 15.07 & $19.19^{*}$ & 160,90 \\
\hline 2 & 13.02 & $17.06^{*}$ & 19.19 & 140,130 \\
\hline 3 & 15 & 17.03 & 19.19 & 130,80 \\
\hline
\end{tabular}




\begin{tabular}{|c|c|c|c|c|}
\hline \multicolumn{5}{|c|}{$\mathrm{N}^{0}$ de equipamentos para monitoramento: $03 \mathrm{un}$. } \\
\hline Solução & \multicolumn{3}{|c|}{ no espaço de objetivos } & no espaço de decisão \\
\hline $\mathrm{N}^{\mathrm{o}}$. & F1 & $\mathrm{F} 2$ & F3 & Nós de monitoramento \\
\hline 1 & 18.05 & $18.14^{*}$ & $19.38^{*}$ & $130,140,90$ \\
\hline 2 & 18.01 & 17.16 & 19.38 & $130,160,90$ \\
\hline 3 & $19.06^{*}$ & 17.09 & 19.38 & $170,160,90$ \\
\hline \multicolumn{5}{|c|}{$\mathrm{N}^{\circ}$ de equipamentos para monitoramento: 04un. } \\
\hline Solução & \multicolumn{3}{|c|}{ no espaço de objetivos } & no espaço de decisão \\
\hline $\mathrm{N}^{\mathrm{o}}$. & F1 & $\mathrm{F} 2$ & F3 & Nós de monitoramento \\
\hline 1 & 19.13 & 18.2 & $19.57 *$ & $160,140,170,90 * *$ \\
\hline 2 & 19.09 & $19.17 *$ & 19.57 & $140,130,170,90$ \\
\hline 3 & 19.14 & 18.19 & 19.57 & $160,130,170,90$ \\
\hline 4 & $19.17 *$ & 17.22 & 19.57 & $160,165,170,90$ \\
\hline \multicolumn{5}{|c|}{$\begin{array}{l}\text { Obs.: *valores máximos de F1, F2 e F3 utilizados nos gráficos } \\
\text { **solução apontada por KESSLER et al. (1998) }\end{array}$} \\
\hline
\end{tabular}

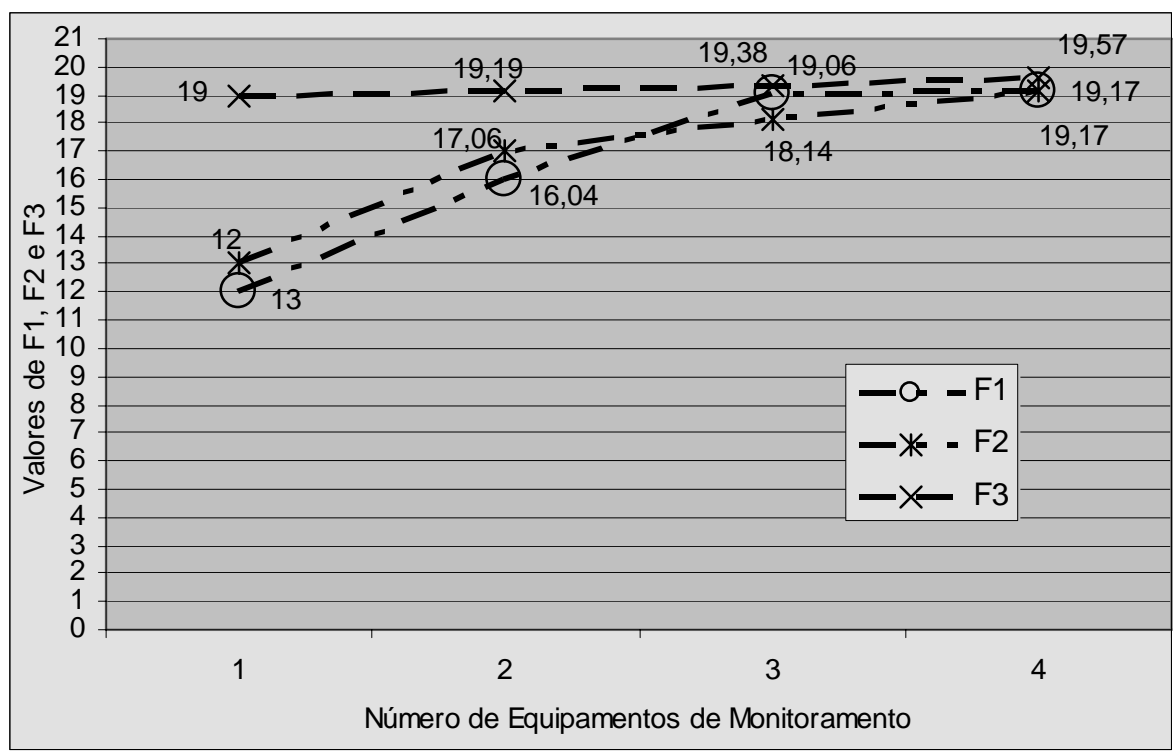

Figura 7.6 - Gráfico Valores máximos de F1, F2 e F3 x Número de Equipamentos para Rede Anytown considerando deteç̧ões repetidas $\left(\mathrm{VC}=1245,15 \mathrm{~m}^{3}\right)$.

Observe que os resultados considerando detecções repetidas são superiores aos resultados sem a consideração, pois mostram mais informações (número de nós já monitorados) que podem ser decisivas ao escolher as estações de monitoramento. 


\section{CONCLUSÕES}

Analisando o funcionamento de redes de distribuição de água para abastecimento, é possível verificar que estes sistemas são vulneráveis a intrusões intencionais de contaminantes, pela facilidade de acesso aos pontos da rede, que pode acarretar sérios danos à saúde pública. Daí a necessidade de se implantar uma rede de monitoramento da qualidade da água em tais sistemas.

Dentre as dificuldades envolvidas no monitoramento da qualidade da água em redes de distribuição, como o comportamento hidráulico, falta de dados e levantamento da rede, dentre outros, destaca-se o estabelecimento da rede de monitoramento e, nesse sentido, a literatura apresenta diversas propostas. O presente estudo propõe um método, cuja aplicação é aqui demonstrada ilustrativamente pela rede Net1 e pela Rede Anytown, que são muito difundidas na literatura.

O desenvolvimento do estudo implicou na codificação e implementação de dois programas computacionais, PLEDECORDA_EPA e PLEDECORDA_MOM, os quais podem ser utilizados para localização dos equipamentos para detecção de contaminação em uma rede qualquer. Ambos os programas obtiveram tempos de processamento curtos para as redes estudadas.

O primeiro programa, PLEDECORDA_EPA, é capaz de gerar as matrizes Custo e Roteamento, utilizadas para montar as redes auxiliares que facilitam o entendimento do problema, e as matrizes poluição para os três níveis de serviço. 
O segundo programa, PLEDECORDA_MOM, é capaz de gerar as Frentes de Pareto (soluções) do problema, que através da investigação das três funções objetivo aplicada às matrizes poluição, disponibiliza diversas opções para se estabelecer a rede de monitoramento dentro dos níveis de serviço sugeridos.

Os resultados mostram que é possível produzir resultados que considerem objetivos múltiplos e, ao mesmo tempo, privilegiem as soluções que possibilitam detecções de mesmas fontes por diversos equipamentos de monitoramento, ampliando a confiabilidade do sistema em casos de eventuais falhas dos equipamentos de monitoramento, ou ainda, é possível que a detecção distancie dos limites do níveis de serviço, ou seja, a detecção para o nível de serviço para Tempo de Detecção de limite de 6 horas, há maiores chances de ocorrer mais rapidamente, em 4 horas, por exemplo. Além disso, com a consideração de detecções repetidas, é possível verificar os objetivos que seus determinados níveis de serviço podem ser diminuídos e assim, aumentar a capacidade da rede de monitoramento.

Verifica-se também, que alguns resultados podem ou às vezes devem ser descartados por certos fatores como: a proximidade entre nós, que acarreta o aumento de detecções repetidas e assim, uma provável solução nãodominada, e pontos na rede de difícil acesso ou de difícil instalação de um equipamento de monitoramento, como por exemplo, uma avenida de grande movimento. Como sugestão, pode ser aplicado o método proposto a um sistema 
com mapeamento dos consumidores, podendo assim, considerar probabilidades de cada nó.

Os níveis de serviço adotados podem ainda ser melhor investigados e variados, conforme a necessidade para cada rede e concessionária, bem como a adição de novos níveis de serviço. Ao invés da rede auxiliar representativa das 24 horas diárias, matrizes de poluição podem ser construídas para os padrões de demanda de cada intervalo horário. Outra sugestão é empregar técnicas de amostragem para criar possíveis combinações de padrões de injeção de contaminantes, etc.

Deve-se ainda ressaltar as limitações para este estudo: as partículas são consideradas conservativas e se propagam na velocidade da água no tubo, não é possível testar intrusão de contaminantes em uma rede real e equipamentos de monitoramento em tempo real para contaminantes são inexistentes, entretanto, com a necessidade, o desenvolvimento de protótipos já é uma realidade.

Para aperfeiçoamento do trabalho, o mapeamento da vulnerabilidade dos sistemas quanto a intrusão de contaminantes em relação a variáveis como acesso aos pontos da rede, tempo de vida dos materiais que compõe a rede e regiões com maiores vazamentos, dá suporte para associar probabilidades de intrusão aos pontos e consequentemente, além de considerar a intrusão intencional de forma mais eficiente, considerar a intrusão acidental de contaminantes. 


\section{REFERÊNCIAS}

- AL-ZAHRANI, M. A., e MOIED, K. “Locating optimum water quality monitoring stations in water distribution system." Bridging the gap: Meeting the world's water and environmental resources challenges, State of the Practice-Proc., World Water and Environmental Resources Congress, CD-ROM, ASCE, Reston, Va., 2001.

- BERRY, J., FLEISCHER, L., HART, W., e PHILliPS, C. "Sensor Placement in Municipal Water Networks." Proc. World Water and Environmental Resources Congress, Philadelphia, PA., 2003.

- CAlderaro, R. V. V., e HELlER, L. "Surto de reações hemolíticas associado a residuais de cloro e cloraminas na água de hemodiálise" Em: Rev. Saúde Pública, v. 35, n. 5, São Paulo, 2001.

- CAVAlCANTI, A. D. "Monitoramento da contaminação por elementos traço em ostras comercializadas em Recife, Pernambuco, Brasil” Em: Cad. Saúde Pública, v.19, n. 5, Rio de Janeiro, 2003.

- CHEUnG, P. B. “Análise de Reabilitação de Redes de Distribuição de Água para Abastecimento via Algoritmos Genéticos Multiobjetivo." Tese (Doutorado) - Escola de Engenharia de São Carlos, Universidade de São Paulo, 268p, 2004.

- CHEUnG, P. B., REIS, L. F. R., FORMiGA, K. T., CHAUdHRY, F. H., TICONA, W. G. C. "Multiobjective Evolutionary Algorithms Applied to the Rehabilitation of a Water Distribution System: A Comparative Study." In: Evolucionary Multi-Criterion Optimization. 1 ed. Berlin Heidelberg: Spring Verlag, v.2532, p. 662-676, 2003.

- CHRISTOFIDES, N. Graph theory: An algorithmic approach. Academic Press, Inc., San Diego, Calif., 1975. 
- COHON, J. L. Multiobjective Programming and Planning. San Diego, Academic, USA, 1978.

- DAVIDSON, J., BOUChART, F., CAVILl, S. e JOWITT, P. "Real-Time Connectivity Modeling of Water Distribution Networks to Predict Contamination Spread" Journal of Computing in Civil Engineering, v. 19, n. 4, p. 377-386, 2005.

- DEB, K. Multi-Objective Using Evolutionary Algorithms. John Wiley \& Sons, Ltd, 2001.

- DECRETO 5440 de 04 de maio de 2005 - Senado Federal, Subsecretaria de Informações.

- DI BERNARDO, L. Métodos e Técnicas de Tratamento de Água. ABES, v.1, Rio de Janeiro, 1983

- FLOYD, R. W. “Algorithm 97: Shortest path." Communications of ACM, 5, 345,1962 .

- FluCK, S., MCKANE, W., CAIRNS, T., FAIRCHILD, V., LAWRENCE A., e LEE J. Chloramine-induced haemolysis presenting as erythropoietin resistance. Nephrol Dial Transplant 1999;14:1687-91.

- GALAL-GORCHEV, H. "Desinfección del agua potable y subproductos de inter's para la salud." In: La calidad del agua potable en America Latina: Ponderación de los riesgos microbiológicos contra los riegos de los subproductos de la desinfeccíon química, Craun, G.F. e Castro, R., eds., p. 89-100. ILSI Press: Washigton, EUA, 1996.

- GESSLER, J., e WALSKI, T. M. "WADISO - Water distribution system optimization.” TREL-11, WES, Corps. of Engineers, Vicksburg, Miss., 1985.

- GOLDBERG, D.E.: Genetic Algorithms in Search, Optimization, and Machine Learning. Addison-Wesley, Reading, Massachusetts, 1989. 
- HARMANT, P., NACE, A., KIENE, L., e FOTOOHI, F. "Optimal supervision of drinking water distribution network." Proc., 26th Annual Water Resources Planning and Management Conf. '99-Preparing for the 21st Century, Cap. 8F180, CD-ROM, ASCE, Reston, Va., 1999.

- JASZKIEWICZ, A. Multiobjective Methods Metaheuristic Library for C++. http://wwwidss.cs.put.poznan.pl/ jaszkiewicz/MOMHLIB/.

- KESSlER, A., e OSTFELD, A. "Detecting accidental contaminations in municipal water networks: Application." Water Resources and the Urban Environment, Proc., 25th Annual Conf. on Water Resources Planning and Management, ASCE, New York, p. 272-278, 1998.

- KESSler, A., OSTFELD, A., e SINAI, G. "Detecting accidental contaminations in municipal water networks." Journal of Water Res. Planning and Management, v. 124, p. 192-198, 1998.

- KUMAR, A., KANSAL, M.L., e ARORA, G. Discussion of "Detecting accidental contaminations in municipal water networks". Journal of Water Resources Planning and Management, v.124, n. 4, p. 308-310, 1999.

- KUMAR, A., KANSAL, M. L., e ARORA, G. "Identification of monitoring stations in water distribution system." Journal of Environmental Engineering, v. 123, n. 8, p. 746-752, 1997.

- LEE, B. H., e DEININGER, R. A. "Optimal locations of monitoring stations in water distribution system." Journal of Environmental Engineering, v. 118, n. 1, p. 4-16, 1992.

- Ostfeld, A., e KESSlER, A. Closure of Discussion of "Detecting accidental contaminations in municipal water networks". Journal of Water Resources Planning and Management, v.124, n. 4, p. 308-310, 1999. 
- OSTFELD, A., e KESSLER, A. "Protecting urban water distribution systems against accidental hazards intrusions". Proc., IWA $2^{\text {nd }}$ Conf., CD-ROM, IWA, 2001.

- OSTFElD, A., e SALOMONS, E. "Optimal Layout of Early Warning Detection Stations for Water Distribution Systems Security" Journal of Water Resources Planning and Management, v. 130, n. 5, p. 377-385, 2004.

- PORTARIA $\mathrm{n}^{\circ}$. 518 de 25 de março de 2004 - Ministério da Saúde. Regulamenta a Norma de qualidade.

- ROSSMAN, L. A. EPANET 2 users manual. U.S. Environmental Protection Agency, Cincinnati, Ohio, 2000.

- TRYBY, M. E., e UBER, J. G. "Representative water quality sampling in water distribution systems." Bridging the gap: Meeting the world's water and environmental resources challenges, State of the Practice-Proc., World Water and Environmental Resources Congress, CD-ROM, ASCE, Reston, Va., 2001.

- VON SPERLING, M. Introdução à Qualidade das águas e ao Tratamento de Esgotos. - 2ed. - Belo Horizonte: Departamento de Engenharia Sanitária e Ambiental, Universidade Federal de Minas Gerais, 1996.

- WAlSKI, T. M., BRILL, E. D., GESSler, J., GOULTER, I. C., JEPPSON, R. M., LANSEY, K., HAN-LIN LEE, LIEBMAN, J. C., Mays, L., MORGAN, D. R., e ORMSBEE, L. "Battle of the network models: Epilogue." Journal of Water Resources Planning and Management, v. 113, n. 2, p. 191-203, 1987.

- WAtson, J. P., GREEnBERG, H. J., HART, W. E. “A Multiple-Objective Analysis of Sensor Placement Optimization in Water Networks." Proc. World Water and Environmental Resources Congress, Salt Lake City, Utah, USA, 2004. 
- WOO, H.-M., YOON, J.-H., e CHOI, D.-Y. “Optimal monitoring sites based on water quality and quantity in water distribution systems." Bridging the gap: Meeting the world's water and environmental resources challenges, State of the Practice-Proc., World Water and Environmental Resources Congress, CD-ROM, ASCE, Reston, Va., 2001.

- ZITZLER, E., THIELE, L. "Multiobjective Evolutionary Algorithms: A Comparative Case Study and the Strength Pareto Approach." IEEE Transactions on Evolutionary Computation, v. 3, n. 4, p. 257-271, 1999.

- ZITZLER, E., THIELE, L. “An Evolutionary Algorithm for Multiobjective Optimization: The Strength Pareto Approach.” Technical Report, 43, Zürich. Switzerland: Computer Engineering and Networks Laboratory (TIK), Swiss Federal Institute of Technology, 1998. 


\section{Anexo I - REDE.INP (Rede Net1): Dados de Entrada}

[TITLE]

EPANET Example Network 1

A simple example of modeling chlorine decay. Both bulk and wall reactions are included.

$\begin{array}{llll}{[\text { JUNCTIONS] }} & & & \\ ; \text { ID } & \text { Elev } & \text { Demand } & \text { Pattern } \\ 10 & 710 & 0 & \\ 11 & 710 & 150 & ; \\ 12 & 700 & 150 & ; \\ 13 & 695 & 100 & ; \\ 21 & 700 & 150 & ; \\ 22 & 695 & 200 & ; \\ 23 & 690 & 150 & ; \\ 31 & 700 & 100 & ; \\ 32 & 710 & 100 & \end{array}$

\section{[RESERVOIRS]}

$\begin{array}{lll}\text {;ID } & \text { Head } & \text { Pattern } \\ 9 & 800 & \end{array}$

[TANKS]

$\begin{array}{llllllll}\text {;ID } & \text { Elevation } & \text { InitLevel } & \text { MinLevel } & \text { MaxLevel } & \text { Diameter } & \text { MinVol } & \text { VolCurve } \\ 2 & 850 & 120 & 100 & 150 & 50.5 & 0 & \end{array}$;

$\begin{array}{lllllrll}\text { [PIPES] } & & & & & \\ ; \text { ID } & \text { Node1 } & \text { Node2 } & \text { Length } & \text { Diameter } & \text { Roughness } & \text { MinorLoss } & \text { Status } \\ 10 & 10 & 11 & 10530 & 18 & 100 & 0 & \text { Open ; } \\ 11 & 11 & 12 & 5280 & 14 & 100 & 0 & \text { Open ; } \\ 12 & 12 & 13 & 5280 & 10 & 100 & 0 & \text { Open ; } \\ 21 & 21 & 22 & 5280 & 10 & 100 & 0 & \text { Open ; } \\ 22 & 22 & 23 & 5280 & 12 & 100 & 0 & \text { Open ; } \\ 31 & 31 & 32 & 5280 & 6 & 100 & 0 & \text { Open ; } \\ 110 & 2 & 12 & 200 & 18 & 100 & 0 & \text { Open ; } \\ 111 & 11 & 21 & 5280 & 10 & 100 & 0 & \text { Open ; } \\ 112 & 12 & 22 & 5280 & 12 & 100 & 0 & \text { Open ; } \\ 113 & 13 & 23 & 5280 & 8 & 100 & 0 & \text { Open ; } \\ 121 & 21 & 31 & 5280 & 8 & 100 & 0 & \text { Open ; } \\ 122 & 22 & 32 & 5280 & 6 & 100 & 0 & \end{array}$

[PUMPS]

;ID $\quad$ Node1 $\quad$ Node2 $\quad$ Parameters

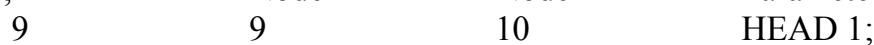

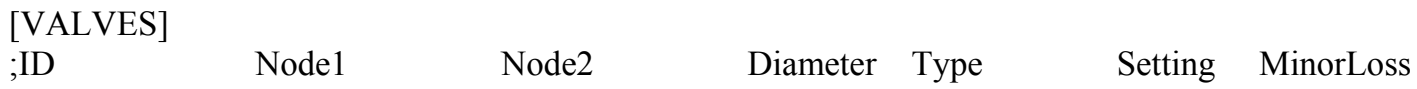

[TAGS]

[DEMANDS]

;Junction

Demand Pattern Category

[STATUS]

;ID Status/Setting 


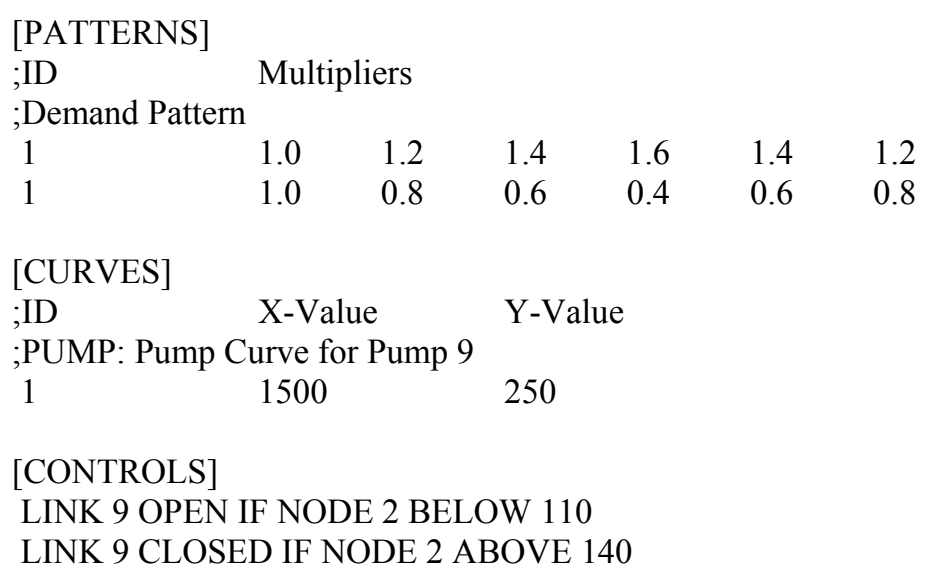

[RULES]

$\begin{array}{ll}\text { [ENERGY] } & \\ \text { Global Efficiency } & 75 \\ \text { Global Price } & 0.0 \\ \text { Demand Charge } & 0.0\end{array}$

[EMITTERS]

;Junction Coefficient

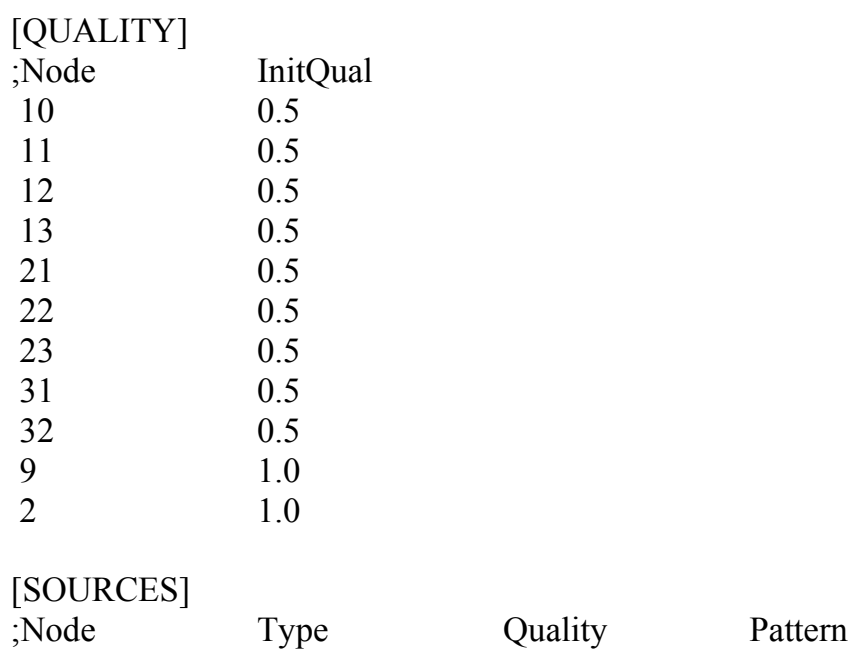

[REACTIONS]

;Type Pipe/Tank Coefficient

[REACTIONS]

Order Bulk

Order Tank

Order Wall 1

Global Bulk $\quad-.5$

Global Wall -1

Limiting Potential $\quad 0.0$

Roughness Correlation $\quad 0.0$

[MIXING] 
Estudo da Localização Otimizada de Equipamentos em Rede de Distribuição de Água para Deteç̧ão de Contaminação

;Tank Model

\begin{tabular}{|c|c|}
\hline [TIMES] & \\
\hline Duration & $240: 00$ \\
\hline Hydraulic Timestep & $1: 00$ \\
\hline Quality Timestep & 0:05 \\
\hline Pattern Timestep & $2: 00$ \\
\hline Pattern Start & 0:00 \\
\hline Report Timestep & $1: 00$ \\
\hline Report Start & 0:00 \\
\hline Start ClockTime & 0:00 \\
\hline Statistic & None \\
\hline [REPORT] & \\
\hline Status & Yes \\
\hline Summary & No \\
\hline Page & 0 \\
\hline [OPTIONS] & \\
\hline Units & GPM \\
\hline Headloss & $\mathrm{H}-\mathrm{W}$ \\
\hline Specific Gravity & 1.0 \\
\hline Viscosity & 1.0 \\
\hline Trials & 40 \\
\hline Accuracy & 0.001 \\
\hline Unbalanced & Continue 10 \\
\hline Pattern & 1 \\
\hline Demand Multiplier & 1.0 \\
\hline Emitter Exponent & 0.5 \\
\hline Quality & Chlorine $\mathrm{mg} / \mathrm{L}$ \\
\hline Diffusivity & 1.0 \\
\hline Tolerance & 0.01 \\
\hline
\end{tabular}

[COORDINATES]

$\begin{array}{lcc}\text {;Node } & \text { X-Coord } & \text { Y-Coord } \\ 10 & 20.00 & 70.00 \\ 11 & 30.00 & 70.00 \\ 12 & 50.00 & 70.00 \\ 13 & 70.00 & 70.00 \\ 21 & 30.00 & 40.00 \\ 22 & 50.00 & 40.00 \\ 23 & 70.00 & 40.00 \\ 31 & 30.00 & 10.00 \\ 32 & 50.00 & 10.00 \\ 9 & 10.00 & 70.00 \\ 2 & 50.00 & 90.00 \\ & & \\ \text { [VERTICES] } & & \\ \text {;Link } & \text { X-Coord } & \text { Y-Coord } \\ & & \\ \text { [LABELS] } & & \\ \text {;X-Coord } & \text { Y-Coord } & \text { Label \& Anchor Node } \\ \text { 6.12 } & 67.96 & \text { "Source" } \\ \text { 13.03 } & 68.08 & \text { "Pump" } \\ 43.85 & 91.21 & \text { "Tank" }\end{array}$

[BACKDROP] 
Estudo da Localização Otimizada de Equipamentos em Rede de Distribuição de Água para Deteç̧ão de Contaminação

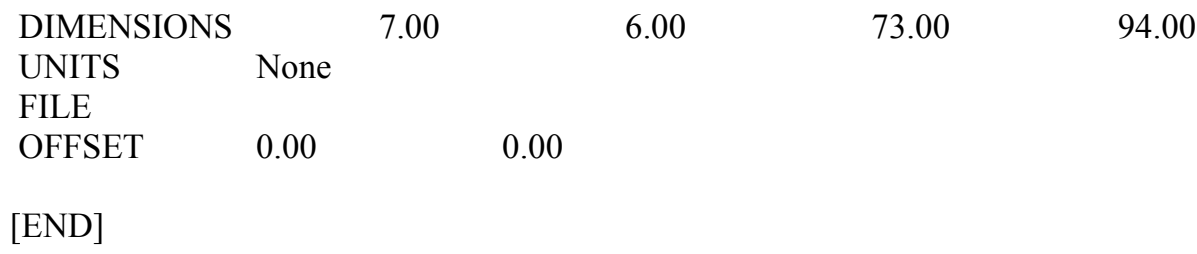




\section{Anexo II - PLEDECORDA_EPA.TXT (Rede Net1): Saídas das Matrizes de Poluição}

PLEDECORDA - Programa de Localização de Equipamentos para DEtecção de COntaminação em Rede de Distribuição de Água

Programador: Luiz Fernando de Souza Dias.

Orientadora: Luisa Fernanda Ribeiro Reis.

Data Inicial do Programa: 15/03/2005

Data e hora do início da execução: 02/16/06 17:12:44

Número de tubos da rede: 12

Número de nós da rede: 11

Número de reservatórios e tanques da rede: 2

Duração do período da Simulação (h): 240

Comprimento total da rede (ft): 63530

Valor do Nível de Serviço 1 - Volume Consumido: $283.17 \mathrm{~m} 3$.

Valor do Nível de Serviço 2 - Tempo de Detecção: 6h.

Valor do Nível de Serviço 3 - Extensão da Contaminação: 15\% do comp. da rede.

TUBO (1): 10 Nó inicial (1): 10 Nó final (2) : 11 Compr.(ft): 10530.00 Diam.(in): 18.00

* Soma+: 1191149.25

* Soma-: $\quad-0.35$

* Contador: 241

TUBO (2): 11 Nó inicial (2): 11 Nó final (3) : 12 Compr.(ft): 5280.00 Diam.(in): 14.00

* Soma+: 1245481.50

* Soma-: -187693.89

* Contador: 241

TUBO (3): 12 Nó inicial (3): 12 Nó final (4) : 13 Compr.(ft): 5280.00 Diam.(in): 10.00

* Soma+: 556701.19

* Soma-: 0.00

* Contador: 241

TUBO (4): 21 Nó inicial (5): 21 Nó final (6) : 22 Compr.(ft): 5280.00 Diam.(in): 10.00

* Soma+: 319494.00

* Soma-: -72170.35

* Contador: 241

TUBO (5): 22 Nó inicial (6): 22 Nó final (7) : 23 Compr.(ft): 5280.00 Diam.(in): 12.00

* Soma+: 228748.44

* Soma-: 0.00

* Contador: 241

TUBO (6): 31 Nó inicial (8): 31 Nó final (9) : 32 Compr.(ft): 5280.00 Diam.(in): 6.00 
Estudo da Localização Otimizada de Equipamentos em Rede de Distribuição de Água para Deteç̧ão de Contaminação

* Soma+: 336050.84

* Soma-: 0.00

* Contador: 241

TUBO (7): 110 Nó inicial (11): 2 Nó final (3) : 12 Compr.(ft): 200.00 Diam.(in): 18.00

* Soma+: 348520.41

* Soma-: -336325.91

* Contador: 241

TUBO (8): 111 Nó inicial (2): 11 Nó final (5) : 21 Compr.(ft): 5280.00 Diam.(in): 10.00

* Soma+: 1254400.38

* Soma-: 0.00

* Contador: 241

TUBO (9): 112 Nó inicial (3): 12 Nó final (6) : 22 Compr.(ft): 5280.00 Diam.(in): 12.00

* Soma+: 711948.38

* Soma-: $\quad-549.29$

* Contador: 241

TUBO (10): 113 Nó inicial (4): 13 Nó final (7) : 23 Compr.(ft): 5280.00 Diam.(in): 8.00

* Soma+: 316405.84

* Soma-: $\quad-372.54$

* Contador: 241

TUBO (11): 121 Nó inicial (5): 21 Nó final (8) : 31 Compr.(ft): 5280.00 Diam.(in): 8.00

* Soma+: 742840.12

* Soma-: 0.00

* Contador: 241

TUBO (12): 122 Nó inicial (6): 22 Nó final (9) : 32 Compr.(ft): 5280.00 Diam.(in): 6.00

* Soma+: 648503.44

* Soma-: 0.00

* Contador: 241

TEMPO MÉDIO DE VIAGEM - SENTIDO ADOTADO:

$\begin{array}{ll}\text { TUBO 10: } & 2.13 \\ \text { TUBO 11: } & 1.02 \\ \text { TUBO 12: } & 2.29 \\ \text { TUBO 21: } & 3.98 \\ \text { TUBO 22: } & 5.56 \\ \text { TUBO 31: } & 3.79 \\ \text { TUBO 110: } & 0.14 \\ \text { TUBO 111: } & 1.01 \\ \text { TUBO 112: } & 1.79 \\ \text { TUBO 113: } & 4.02 \\ \text { TUBO 121: } & 1.71 \\ \text { TUBO 122: } & 1.96\end{array}$

TEMPO MÉDIO DE VIAGEM - SENTIDO INVERSO:

TUBO 10: 0.00

TUBO 11: 6.78 
Estudo da Localização Otimizada de Equipamentos em Rede de Distribuição de Água para Deteç̧ão de Contaminação

TUBO 12: $\quad 0.00$

TUBO 21: $\quad 17.63$

TUBO 22: $\quad 0.00$

TUBO 31: $\quad 0.00$

TUBO 110: $\quad 0.14$

TUBO 111: $\quad 0.00$

TUBO 112: $\quad 0.00$

TUBO 113: $\quad 0.00$

TUBO 121: $\quad 0.00$

TUBO 122: $\quad 0.00$

Matriz Custo de Tempo de viagem Inicial (h):

\begin{tabular}{|c|ccccccccccc|}
\hline Nós: & 10 & 11 & 12 & 13 & 21 & 22 & 23 & 31 & 32 & 9 & 2 \\
\hline 10 & 0 & 2.13 & 888 & 888 & 888 & 888 & 888 & 888 & 888 & 888 & 888 \\
11 & 888 & 0 & 1.02 & 888 & 1.01 & 888 & 888 & 888 & 888 & 888 & 888 \\
12 & 888 & 6.78 & 0 & 2.29 & 888 & 1.79 & 888 & 888 & 888 & 888 & 0.14 \\
13 & 888 & 888 & 888 & 0 & 888 & 888 & 4.02 & 888 & 888 & 888 & 888 \\
21 & 888 & 888 & 888 & 888 & 0 & 3.98 & 888 & 1.71 & 888 & 888 & 888 \\
22 & 888 & 888 & 888 & 888 & 17.63 & 0 & 5.56 & 888 & 1.96 & 888 & 888 \\
23 & 888 & 888 & 888 & 888 & 888 & 888 & 0 & 888 & 888 & 888 & 888 \\
31 & 888 & 888 & 888 & 888 & 888 & 888 & 888 & 0 & 3.79 & 888 & 888 \\
32 & 888 & 888 & 888 & 888 & 888 & 888 & 888 & 888 & 0 & 888 & 888 \\
9 & 0 & 888 & 888 & 888 & 888 & 888 & 888 & 888 & 888 & 0 & 888 \\
2 & 888 & 888 & 0.14 & 888 & 888 & 888 & 888 & 888 & 888 & 888 & 0 \\
\hline
\end{tabular}

Matriz Roteamento Inicial 1:

\begin{tabular}{|c|ccccccccccc|}
\hline Nós: & 10 & 11 & 12 & 13 & 21 & 22 & 23 & 31 & 32 & 9 & 2 \\
\hline 10 & 1 & 2 & 0 & 0 & 0 & 0 & 0 & 0 & 0 & 0 & 0 \\
11 & 0 & 2 & 3 & 0 & 5 & 0 & 0 & 0 & 0 & 0 & 0 \\
12 & 0 & 2 & 3 & 4 & 0 & 6 & 0 & 0 & 0 & 0 & 11 \\
13 & 0 & 0 & 0 & 4 & 0 & 0 & 7 & 0 & 0 & 0 & 0 \\
21 & 0 & 0 & 0 & 0 & 5 & 6 & 0 & 8 & 0 & 0 & 0 \\
22 & 0 & 0 & 0 & 0 & 5 & 6 & 7 & 0 & 9 & 0 & 0 \\
23 & 0 & 0 & 0 & 0 & 0 & 0 & 7 & 0 & 0 & 0 & 0 \\
31 & 0 & 0 & 0 & 0 & 0 & 0 & 0 & 8 & 9 & 0 & 0 \\
32 & 0 & 0 & 0 & 0 & 0 & 0 & 0 & 0 & 9 & 0 & 0 \\
9 & 1 & 0 & 0 & 0 & 0 & 0 & 0 & 0 & 0 & 10 & 0 \\
2 & 0 & 0 & 3 & 0 & 0 & 0 & 0 & 0 & 0 & 0 & 11 \\
\hline
\end{tabular}


Estudo da Localização Otimizada de Equipamentos em Rede de Distribuição de Água para Deteç̧ão de Contaminação

Matriz Custo de Tempo de viagem Final (h) (Caminhos Mais Curtos):

\begin{tabular}{|c|ccccccccccc|}
\hline Nós: & 10 & 11 & 12 & 13 & 21 & 22 & 23 & 31 & 32 & 9 & 2 \\
\hline 10 & 0 & 2.13 & 3.15 & 5.44 & 3.14 & 4.94 & 9.46 & 4.86 & 6.90 & 888 & 3.30 \\
11 & 888 & 0 & 1.02 & 3.31 & 1.01 & 2.81 & 7.33 & 2.73 & 4.77 & 888 & 1.16 \\
12 & 888 & 6.78 & 0 & 2.29 & 7.79 & 1.79 & 6.31 & 9.51 & 3.75 & 888 & 0.14 \\
13 & 888 & 888 & 888 & 0 & 888 & 888 & 4.02 & 888 & 888 & 888 & 888 \\
21 & 888 & 888 & 888 & 888 & 0 & 3.98 & 9.55 & 1.71 & 5.50 & 888 & 888 \\
22 & 888 & 888 & 888 & 888 & 17.63 & 0 & 5.56 & 19.34 & 1.96 & 888 & 888 \\
23 & 888 & 888 & 888 & 888 & 888 & 888 & 0 & 888 & 888 & 888 & 888 \\
31 & 888 & 888 & 888 & 888 & 888 & 888 & 888 & 0 & 3.79 & 888 & 888 \\
32 & 888 & 888 & 888 & 888 & 888 & 888 & 888 & 888 & 0 & 888 & 888 \\
9 & 0 & 2.13 & 3.15 & 5.44 & 3.14 & 4.94 & 9.46 & 4.86 & 6.90 & 0 & 3.30 \\
2 & 888 & 6.92 & 0.14 & 2.42 & 7.93 & 1.93 & 6.45 & 9.65 & 3.89 & 888 & 0 \\
\hline
\end{tabular}

Matriz Roteamento Final 1:

\begin{tabular}{|c|ccccccccccc|}
\hline Nós: & 10 & 11 & 12 & 13 & 21 & 22 & 23 & 31 & 32 & 9 & 2 \\
\hline 10 & 1 & 2 & 2 & 2 & 2 & 2 & 2 & 2 & 2 & 0 & 2 \\
11 & 0 & 2 & 3 & 3 & 5 & 3 & 3 & 5 & 3 & 0 & 3 \\
12 & 0 & 2 & 3 & 4 & 2 & 6 & 4 & 2 & 6 & 0 & 11 \\
13 & 0 & 0 & 0 & 4 & 0 & 0 & 7 & 0 & 0 & 0 & 0 \\
21 & 0 & 0 & 0 & 0 & 5 & 6 & 6 & 8 & 8 & 0 & 0 \\
22 & 0 & 0 & 0 & 0 & 5 & 6 & 7 & 5 & 9 & 0 & 0 \\
23 & 0 & 0 & 0 & 0 & 0 & 0 & 7 & 0 & 0 & 0 & 0 \\
31 & 0 & 0 & 0 & 0 & 0 & 0 & 0 & 8 & 9 & 0 & 0 \\
32 & 0 & 0 & 0 & 0 & 0 & 0 & 0 & 0 & 9 & 0 & 0 \\
9 & 1 & 1 & 1 & 1 & 1 & 1 & 1 & 1 & 1 & 10 & 1 \\
2 & 0 & 3 & 3 & 3 & 3 & 3 & 3 & 3 & 3 & 0 & 11 \\
\hline
\end{tabular}

Matriz Custo de Extensão de viagem Inicial (ft):

\begin{tabular}{|c|ccccccccccc|}
\hline Nós: & 10 & 11 & 12 & 13 & 21 & 22 & 23 & 31 & 32 & 9 & 2 \\
\hline 10 & 0 & 10530 & 63530 & 63530 & 63530 & 63530 & 63530 & 63530 & 63530 & 63530 & 63530 \\
11 & 63530 & 0 & 5280 & 63530 & 5280 & 63530 & 63530 & 63530 & 63530 & 63530 & 63530 \\
12 & 63530 & 5280 & 0 & 5280 & 63530 & 5280 & 63530 & 63530 & 63530 & 63530 & 200 \\
13 & 63530 & 63530 & 63530 & 0 & 63530 & 63530 & 5280 & 63530 & 63530 & 63530 & 63530 \\
21 & 63530 & 63530 & 63530 & 63530 & 0 & 5280 & 63530 & 5280 & 63530 & 63530 & 63530 \\
22 & 63530 & 63530 & 63530 & 63530 & 5280 & 0 & 5280 & 63530 & 5280 & 63530 & 63530 \\
23 & 63530 & 63530 & 63530 & 63530 & 63530 & 63530 & 0 & 63530 & 63530 & 63530 & 63530 \\
31 & 63530 & 63530 & 63530 & 63530 & 63530 & 63530 & 63530 & 0 & 5280 & 63530 & 63530 \\
32 & 63530 & 63530 & 63530 & 63530 & 63530 & 63530 & 63530 & 63530 & 0 & 63530 & 63530 \\
9 & 0 & 63530 & 63530 & 63530 & 63530 & 63530 & 63530 & 63530 & 63530 & 0 & 63530 \\
2 & 63530 & 63530 & 200 & 63530 & 63530 & 63530 & 63530 & 63530 & 63530 & 63530 & 0 \\
\hline
\end{tabular}


Estudo da Localização Otimizada de Equipamentos em Rede de Distribuição de Água para Deteç̧ão de Contaminação

Matriz Roteamento Inicial 2:

\begin{tabular}{|c|ccccccccccc|}
\hline Nós: & 10 & 11 & 12 & 13 & 21 & 22 & 23 & 31 & 32 & 9 & 2 \\
\hline 10 & 1 & 2 & 0 & 0 & 0 & 0 & 0 & 0 & 0 & 0 & 0 \\
11 & 0 & 2 & 3 & 0 & 5 & 0 & 0 & 0 & 0 & 0 & 0 \\
12 & 0 & 2 & 3 & 4 & 0 & 6 & 0 & 0 & 0 & 0 & 11 \\
13 & 0 & 0 & 0 & 4 & 0 & 0 & 7 & 0 & 0 & 0 & 0 \\
21 & 0 & 0 & 0 & 0 & 5 & 6 & 0 & 8 & 0 & 0 & 0 \\
22 & 0 & 0 & 0 & 0 & 5 & 6 & 7 & 0 & 9 & 0 & 0 \\
23 & 0 & 0 & 0 & 0 & 0 & 0 & 7 & 0 & 0 & 0 & 0 \\
31 & 0 & 0 & 0 & 0 & 0 & 0 & 0 & 8 & 9 & 0 & 0 \\
32 & 0 & 0 & 0 & 0 & 0 & 0 & 0 & 0 & 9 & 0 & 0 \\
9 & 1 & 0 & 0 & 0 & 0 & 0 & 0 & 0 & 0 & 10 & 0 \\
2 & 0 & 0 & 3 & 0 & 0 & 0 & 0 & 0 & 0 & 0 & 11 \\
\hline
\end{tabular}

Matriz Custo de Extensão de viagem Final (ft) (Caminhos Mais Curtos):

\begin{tabular}{|c|ccccccccccc|}
\hline Nós: & 10 & 11 & 12 & 13 & 21 & 22 & 23 & 31 & 32 & 9 & 2 \\
\hline 10 & 0 & 10530 & 15810 & 21090 & 15810 & 21090 & 26370 & 21090 & 26370 & 63530 & 16010 \\
11 & 63530 & 0 & 5280 & 10560 & 5280 & 10560 & 15840 & 10560 & 15840 & 63530 & 5480 \\
12 & 63530 & 5280 & 0 & 5280 & 10560 & 5280 & 10560 & 15840 & 10560 & 63530 & 200 \\
13 & 63530 & 63530 & 63530 & 0 & 63530 & 63530 & 5280 & 63530 & 63530 & 63530 & 63530 \\
21 & 63530 & 63530 & 63530 & 63530 & 0 & 5280 & 10560 & 5280 & 10560 & 63530 & 63530 \\
22 & 63530 & 63530 & 63530 & 63530 & 5280 & 0 & 5280 & 10560 & 5280 & 63530 & 63530 \\
23 & 63530 & 63530 & 63530 & 63530 & 63530 & 63530 & 0 & 63530 & 63530 & 63530 & 63530 \\
31 & 63530 & 63530 & 63530 & 63530 & 63530 & 63530 & 63530 & 0 & 5280 & 63530 & 63530 \\
32 & 63530 & 63530 & 63530 & 63530 & 63530 & 63530 & 63530 & 63530 & 0 & 63530 & 63530 \\
9 & 0 & 10530 & 15810 & 21090 & 15810 & 21090 & 26370 & 21090 & 26370 & 0 & 16010 \\
2 & 63530 & 5480 & 200 & 5480 & 10760 & 5480 & 10760 & 16040 & 10760 & 63530 & 0 \\
\hline
\end{tabular}

Matriz Roteamento Final 2:

\begin{tabular}{|c|ccccccccccc|}
\hline Nós: & 10 & 11 & 12 & 13 & 21 & 22 & 23 & 31 & 32 & 9 & 2 \\
\hline 10 & 1 & 2 & 2 & 2 & 2 & 2 & 2 & 2 & 2 & 0 & 2 \\
11 & 0 & 2 & 3 & 3 & 5 & 3 & 3 & 5 & 3 & 0 & 3 \\
12 & 0 & 2 & 3 & 4 & 2 & 6 & 4 & 2 & 6 & 0 & 11 \\
13 & 0 & 0 & 0 & 4 & 0 & 0 & 7 & 0 & 0 & 0 & 0 \\
21 & 0 & 0 & 0 & 0 & 5 & 6 & 6 & 8 & 6 & 0 & 0 \\
22 & 0 & 0 & 0 & 0 & 5 & 6 & 7 & 5 & 9 & 0 & 0 \\
23 & 0 & 0 & 0 & 0 & 0 & 0 & 7 & 0 & 0 & 0 & 0 \\
31 & 0 & 0 & 0 & 0 & 0 & 0 & 0 & 8 & 9 & 0 & 0 \\
32 & 0 & 0 & 0 & 0 & 0 & 0 & 0 & 0 & 9 & 0 & 0 \\
9 & 1 & 1 & 1 & 1 & 1 & 1 & 1 & 1 & 1 & 10 & 1 \\
2 & 0 & 3 & 3 & 3 & 3 & 3 & 3 & 3 & 3 & 0 & 11 \\
\hline
\end{tabular}


Estudo da Localização Otimizada de Equipamentos em Rede de Distribuição de Água para Deteç̧ão de Contaminação

Matriz Poluição para Volume Consumido (nível de serviço: 283.17m3):

\begin{tabular}{|c|ccccccccccc|}
\hline Nós: & 10 & 11 & 12 & 13 & 21 & 22 & 23 & 31 & 32 & 9 & 2 \\
\hline 10 & 0 & 1 & 1 & 0 & 1 & 1 & 0 & 1 & 0 & 0 & 1 \\
11 & 0 & 1 & 1 & 0 & 1 & 1 & 0 & 1 & 0 & 0 & 1 \\
12 & 0 & 0 & 1 & 1 & 0 & 1 & 0 & 0 & 1 & 0 & 1 \\
13 & 0 & 0 & 0 & 1 & 0 & 0 & 1 & 0 & 0 & 0 & 0 \\
21 & 0 & 0 & 0 & 0 & 1 & 1 & 0 & 1 & 0 & 0 & 0 \\
22 & 0 & 0 & 0 & 0 & 0 & 1 & 0 & 0 & 1 & 0 & 0 \\
23 & 0 & 0 & 0 & 0 & 0 & 0 & 1 & 0 & 0 & 0 & 0 \\
31 & 0 & 0 & 0 & 0 & 0 & 0 & 0 & 1 & 1 & 0 & 0 \\
32 & 0 & 0 & 0 & 0 & 0 & 0 & 0 & 0 & 1 & 0 & 0 \\
9 & 1 & 1 & 1 & 0 & 1 & 1 & 0 & 1 & 0 & 0 & 1 \\
2 & 0 & 0 & 1 & 1 & 0 & 1 & 0 & 0 & 1 & 0 & 0 \\
\hline
\end{tabular}

Matriz Poluição para Tempo de Detecção (nível de serviço: 6.00h.)

\begin{tabular}{|c|ccccccccccc|}
\hline Nós: & 10 & 11 & 12 & 13 & 21 & 22 & 23 & 31 & 32 & 9 & 2 \\
\hline 10 & 1 & 1 & 1 & 1 & 1 & 1 & 0 & 1 & 0 & 0 & 1 \\
11 & 0 & 1 & 1 & 1 & 1 & 1 & 0 & 1 & 1 & 0 & 1 \\
12 & 0 & 0 & 1 & 1 & 0 & 1 & 0 & 0 & 1 & 0 & 1 \\
13 & 0 & 0 & 0 & 1 & 0 & 0 & 1 & 0 & 0 & 0 & 0 \\
21 & 0 & 0 & 0 & 0 & 1 & 1 & 0 & 1 & 1 & 0 & 0 \\
22 & 0 & 0 & 0 & 0 & 0 & 1 & 1 & 0 & 1 & 0 & 0 \\
23 & 0 & 0 & 0 & 0 & 0 & 0 & 1 & 0 & 0 & 0 & 0 \\
31 & 0 & 0 & 0 & 0 & 0 & 0 & 0 & 1 & 1 & 0 & 0 \\
32 & 0 & 0 & 0 & 0 & 0 & 0 & 0 & 0 & 1 & 0 & 0 \\
9 & 1 & 1 & 1 & 1 & 1 & 1 & 0 & 1 & 0 & 1 & 1 \\
2 & 0 & 0 & 1 & 1 & 0 & 1 & 0 & 0 & 1 & 0 & 1 \\
\hline
\end{tabular}

Matriz Poluição para Extensão da Contaminação (nível de serviço: 15.00\%):

\begin{tabular}{|c|ccccccccccc|}
\hline Nós: & 10 & 11 & 12 & 13 & 21 & 22 & 23 & 31 & 32 & 9 & 2 \\
\hline 10 & 1 & 0 & 0 & 0 & 0 & 0 & 0 & 0 & 0 & 0 & 0 \\
11 & 0 & 1 & 1 & 0 & 1 & 0 & 0 & 0 & 0 & 0 & 1 \\
12 & 0 & 1 & 1 & 1 & 0 & 1 & 0 & 0 & 0 & 0 & 1 \\
13 & 0 & 0 & 0 & 1 & 0 & 0 & 1 & 0 & 0 & 0 & 0 \\
21 & 0 & 0 & 0 & 0 & 1 & 1 & 0 & 1 & 0 & 0 & 0 \\
22 & 0 & 0 & 0 & 0 & 1 & 1 & 1 & 0 & 1 & 0 & 0 \\
23 & 0 & 0 & 0 & 0 & 0 & 0 & 1 & 0 & 0 & 0 & 0 \\
31 & 0 & 0 & 0 & 0 & 0 & 0 & 0 & 1 & 1 & 0 & 0 \\
32 & 0 & 0 & 0 & 0 & 0 & 0 & 0 & 0 & 1 & 0 & 0 \\
9 & 1 & 0 & 0 & 0 & 0 & 0 & 0 & 0 & 0 & 1 & 0 \\
2 & 0 & 1 & 1 & 1 & 0 & 1 & 0 & 0 & 0 & 0 & 1 \\
\hline
\end{tabular}

Programa finalizado com sucesso!

Tempo decorrido: $6.29 \mathrm{~s}$. 


\section{Anexo III - PLEDECORDA_MOM.TXT (Rede Net1): \\ Saídas dos Nós escolhidos}

\section{FRENTES PARETO GLOBAL}

Número de Equipamentos: 1 un.

Considerado acréscimo de 0.01 por detecções repetidas.
f1: f2: f3:
Nós de monitoramento (X[i]):
$\begin{array}{llll}1: & 7 & 7 & 4\end{array}$
$22(5)$

Número de Equipamentos: 2 un.

Considerado acréscimo de 0.01 por detecções repetidas.
f1: f2: f3:
Nós de monitoramento (X[i]):
1: $\quad 6.02 \quad 10.03 \quad 6$
$13(3) \quad 32(8)$
$\begin{array}{llll}2: & 9.03 & 9.05 & 6.01\end{array}$
$32(8) \quad 22(5)$

Número de Equipamentos: 3 un.

Considerado acréscimo de 0.01 por detecções repetidas.

f1: f2: f3: $\quad$ Nós de monitoramento (X[i]):
1: $\quad 11.03 \quad 11.06 \quad 8.02$
$22(5) \quad 23(6) \quad 32(8)$ 


\section{FRENTES PARETO GLOBAL}

Número de Equipamentos: 1 un.

Não considerado acréscimo por detecções repetidas.
f1: f2: f3
Nós de monitoramento (X[i]):
$\begin{array}{llll}1: & 7 & 7 & 4\end{array}$
$22(5)$

Número de Equipamentos: 2 un.

Não considerado acréscimo por detecções repetidas.
f1: f2: f3:
Nós de monitoramento (X[i]):

$\begin{array}{llllll}\text { 1: } & 9 & 9 & 6 & 23(6) & 22(5) \\ \text { 2: } & 6 & 10 & 6 & 13(3) & 32(8)\end{array}$

Número de Equipamentos: 3 un.

Não considerado acréscimo por detecções repetidas.

f1: f2: f3: $\quad$ Nós de monitoramento (X[i]):

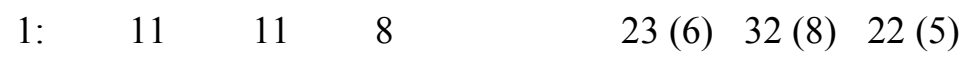




\section{Anexo IV - REDE.INP (Rede Anytown): Dados de Entrada}

[TITLE]

Rede ANYTOWN

[JUNCTIONS]

;ID

20

30

40

50

60

70

80

90

100

110

120

130

140

150

160

170

\section{Elev}

20

50

50

50

50

50

50

50

50

50

120

120

80

120

120

120

Demand
500
200
200
200
500
500
500
1000
500
500
200
200
200
200
800
200

[RESERVOIRS]

$\begin{array}{ll}\text {;ID } & \text { Head } \\ 10 & 10\end{array}$

[TANKS]

$\begin{array}{lllllllc}\text {;ID } & \text { Elevation } & \text { InitLevel } & \text { MinLevel } & \text { MaxLevel } & \text { Diameter } & \text { MinVol } & \text { VolCurve } \\ 65 & 215 & 35 & 10 & 35 & 53.8 & 0 & ; \\ 165 & 215 & 35 & 10 & 35 & 53.8 & 0 & ;\end{array}$

[PIPES]

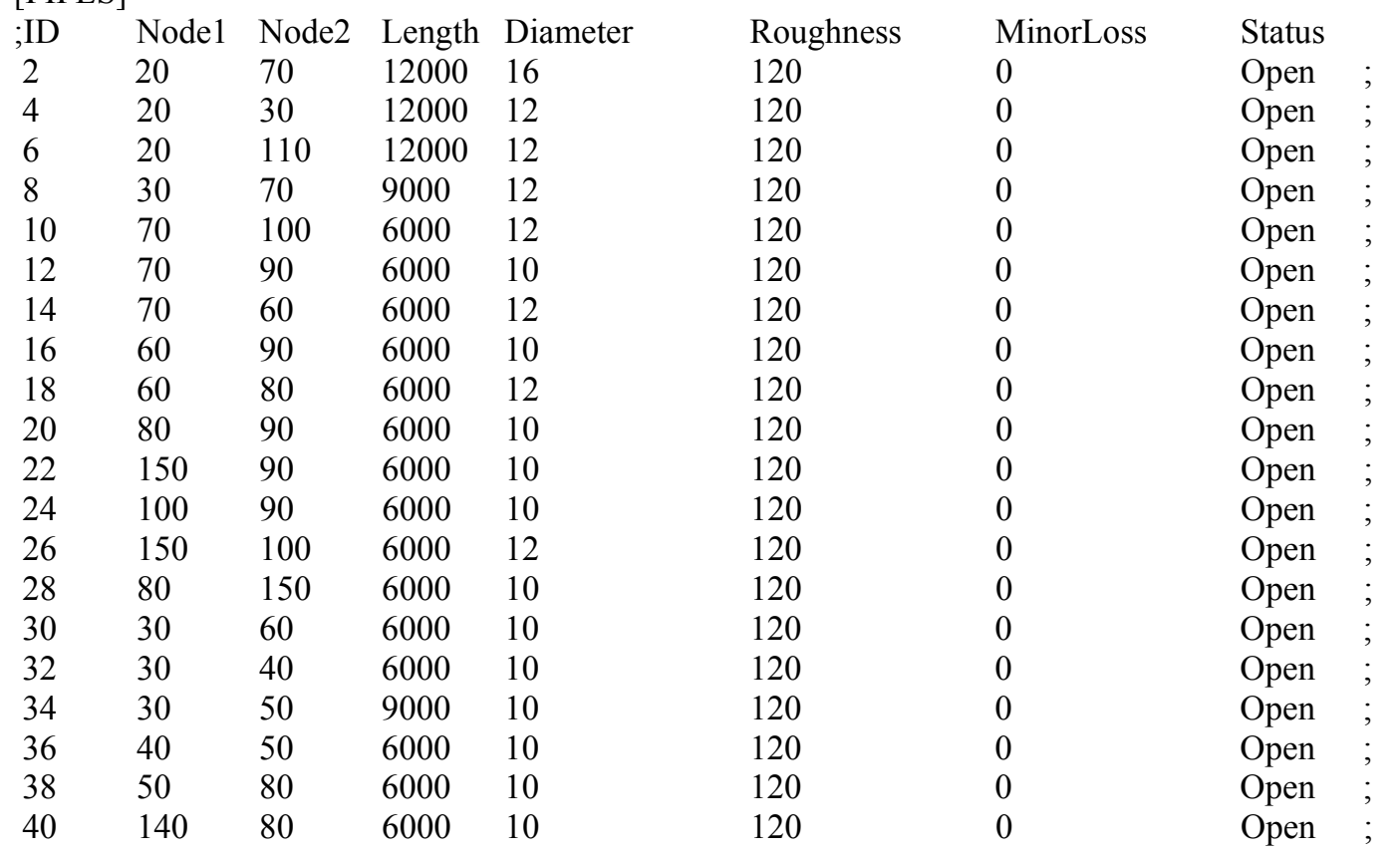


Estudo da Localização Otimizada de Equipamentos em Rede de Distribuição de Água para Deteç̧ão de Contaminação

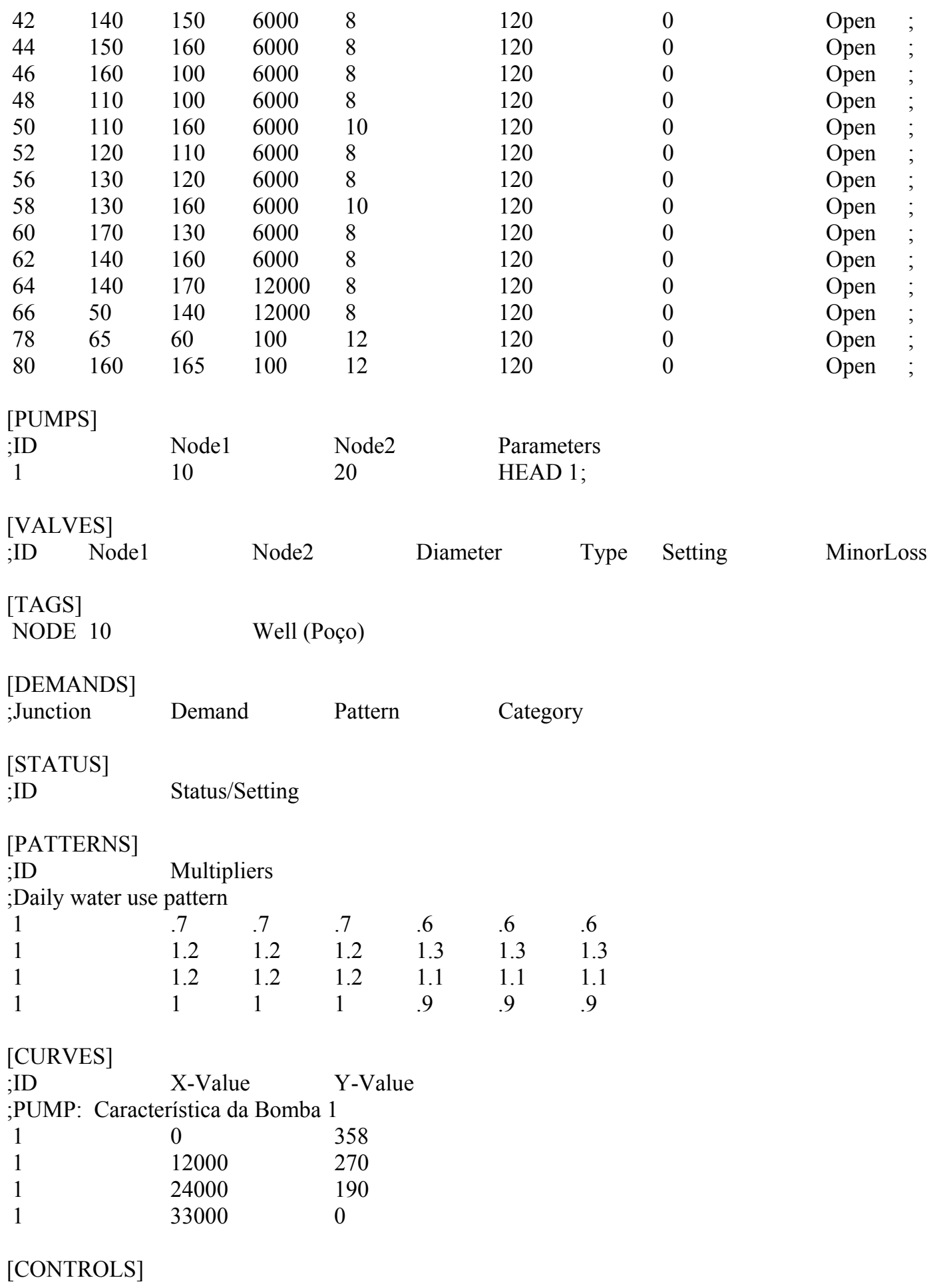

[RULES]

[ENERGY]

Global Efficiency $\quad 75$

Global Price $\quad 0$

Demand Charge $\quad 0$ 
Estudo da Localização Otimizada de Equipamentos em Rede de Distribuição de Água para Deteç̧ão de Contaminação

\begin{tabular}{|c|c|c|c|}
\hline $\begin{array}{l}\text { [EMITTERS] } \\
\text {;Junction }\end{array}$ & Coefficient & & \\
\hline [QUALITY] & & & \\
\hline ;Node & InitQual & & \\
\hline [SOURCES] & & & \\
\hline ;Node & Type & Quality & Pattern \\
\hline [REACTIONS] & & & \\
\hline ;Type & Pipe/Tank & Coefficient & \\
\hline [REACTIONS] & & & \\
\hline Order Bulk & 1 & & \\
\hline Order Tank & 1 & & \\
\hline Order Wall & 1 & & \\
\hline Global Bulk & 0 & & \\
\hline Global Wall & 0 & & \\
\hline Limiting Potential & 0 & & \\
\hline Roughness Correlation & 0 & & \\
\hline [MIXING] & & & \\
\hline ;Tank & Model & & \\
\hline [TIMES] & & & \\
\hline Duration & $240: 00$ & & \\
\hline Hydraulic Timestep & $1: 00$ & & \\
\hline Quality Timestep & $0: 05$ & & \\
\hline Pattern Timestep & $1: 00$ & & \\
\hline Pattern Start & 0:00 & & \\
\hline Report Timestep & 1:00 & & \\
\hline Report Start & 0:00 & & \\
\hline Start ClockTime & $0: 00$ & & \\
\hline Statistic & None & & \\
\hline [REPORT] & & & \\
\hline Status & No & & \\
\hline Summary & No & & \\
\hline Page & 0 & & \\
\hline [OPTIONS] & & & \\
\hline Units & GPM & & \\
\hline Headloss & $\mathrm{H}-\mathrm{W}$ & & \\
\hline Specific Gravity 1 & & & \\
\hline Viscosity & 1 & & \\
\hline Trials & 40 & & \\
\hline Accuracy & 0.001 & & \\
\hline Unbalanced & Continue 10 & & \\
\hline Pattern & 1 & & \\
\hline Demand Multiplier & 1.0 & & \\
\hline Emitter Exponent & 0.5 & & \\
\hline Quality & NONE mg/L & & \\
\hline Diffusivity & 1.0 & & \\
\hline Tolerance & 0.01 & & \\
\hline
\end{tabular}


Estudo da Localização Otimizada de Equipamentos em Rede de Distribuição de Água para Detecção de Contaminação

\begin{tabular}{|c|c|c|c|c|}
\hline \multicolumn{5}{|c|}{ [COORDINATES] } \\
\hline ;Node & X-Coord & \multicolumn{3}{|l|}{ Y-Coord } \\
\hline 20 & 7700.00 & \multicolumn{3}{|l|}{3350.00} \\
\hline 30 & 7700.00 & \multicolumn{3}{|l|}{6000.00} \\
\hline 40 & 7700.00 & \multicolumn{3}{|l|}{7700.00} \\
\hline 50 & 5200.00 & \multicolumn{3}{|l|}{7700.00} \\
\hline 60 & 6500.00 & \multicolumn{3}{|l|}{6000.00} \\
\hline 70 & 6500.00 & \multicolumn{3}{|l|}{4500.00} \\
\hline 80 & 4700.00 & \multicolumn{3}{|l|}{6200.00} \\
\hline 90 & 5500.00 & \multicolumn{3}{|l|}{5300.00} \\
\hline 100 & 5000.00 & \multicolumn{3}{|l|}{4500.00} \\
\hline 110 & 4800.00 & \multicolumn{3}{|l|}{3350.00} \\
\hline 120 & 3000.00 & \multicolumn{3}{|l|}{3100.00} \\
\hline 130 & 2000.00 & \multicolumn{3}{|l|}{4000.00} \\
\hline 140 & 3000.00 & \multicolumn{3}{|l|}{6000.00} \\
\hline 150 & 4000.00 & \multicolumn{3}{|l|}{5200.00} \\
\hline 160 & 3000.00 & 4500.00 & & \\
\hline 170 & 1300.00 & 5000.00 & & \\
\hline 10 & 8720.83 & 3368.11 & & \\
\hline 65 & 6000.00 & 6600.00 & & \\
\hline 165 & 2500.00 & 4700.00 & & \\
\hline [VERTICES] & & & & \\
\hline ;Link & X-Coord & Y-Coord & & \\
\hline [LABELS] & & & & \\
\hline ;X-Coord & Y-Coord & Label \& Anchor Node & & \\
\hline [BACKDROP] & & & & \\
\hline DIMENSIONS & 935.87 & 2613.65 & 9070.35 & 8738.70 \\
\hline UNITS & Meters & & & \\
\hline FILE & & & & \\
\hline OFFSET & 0.00 & 0.00 & & \\
\hline
\end{tabular}




\section{Anexo V - PLEDECORDA_EPA.TXT (Rede Anytown): Saídas das Matrizes de Poluição: Nível de Serviço 1 - Volume Consumido: 468,10m³}

PLEDECORDA - Programa de Localização de Equipamentos para DEtecção de COntaminação em Rede de Distribuição de Água

Programador: Luiz Fernando de Souza Dias.

Orientadora: Luisa Fernanda Ribeiro Reis.

Data Inicial do Programa: 15/03/2005

Data e hora do início da execução: 02/16/06 13:59:51

Número de tubos da rede: 34

Número de nós da rede: 19

Número de reservatórios e tanques da rede: 3

Duração do período da Simulação (h): 240

Comprimento total da rede (ft): 228200

TUBO (1): 2 Nó inicial (1): 20 Nó final (6) : 70 Compr.(ft): 12000.00 Diam.(in): 16.00

* Soma+: 4121963.25

* Soma-: 0.00

* Contador: 241

TUBO (2): 4 Nó inicial (1): 20 Nó final (2) : 30 Compr.(ft): 12000.00 Diam.(in): 12.00

* Soma+: 3453652.50

* Soma-: 0.00

* Contador: 241

TUBO (3): 6 Nó inicial (1): 20 Nó final (10) : 110 Compr.(ft): 12000.00 Diam.(in): 12.00

* Soma+: 3700607.75

* Soma-: 0.00

* Contador: 241

TUBO (4): 8 Nó inicial (2): 30 Nó final (6) : 70 Compr.(ft): 9000.00 Diam.(in): 12.00

* Soma+: $\quad 0.00$

* Soma-: -298116.16

* Contador: 241

TUBO (5): 10 Nó inicial (6): 70 Nó final (9) : 100 Compr.(ft): 6000.00 Diam.(in): 12.00

* Soma+: 2309610.25

* Soma-: 0.00

* Contador: 241

TUBO (6): 12 Nó inicial (6): 70 Nó final (8) : 90 Compr.(ft): 6000.00 Diam.(in): 10.00

* Soma+: 2158205.75 
Estudo da Localização Otimizada de Equipamentos em Rede de Distribuição de Água para Deteç̧ão de Contaminação

* Soma-: $\quad 0.00$

* Contador: 241

TUBO (7): 14 Nó inicial (6): 70 Nó final (5) : 60 Compr.(ft): 6000.00 Diam.(in): 12.00

* Soma+: 1992290.62

* Soma-: 0.00

* Contador: 241

TUBO (8): 16 Nó inicial (5): 60 Nó final (8) : 90 Compr.(ft): 6000.00 Diam.(in): 10.00

* Soma+: 1047277.31

* Soma-: 0.00

* Contador: 241

TUBO (9): 18 Nó inicial (5): 60 Nó final (7) : 80 Compr.(ft): 6000.00 Diam.(in): 12.00

* Soma+: 1189309.25

* Soma-: $\quad 0.00$

* Contador: 241

TUBO (10): 20 Nó inicial (7): 80 Nó final (8) : 90 Compr.(ft): 6000.00 Diam.(in): 10.00

* Soma+: 99212.45

* Soma-: -69697.99

* Contador: 241

TUBO (11): 22 Nó inicial (14): 150 Nó final (8) : 90 Compr.(ft): 6000.00 Diam.(in): 10.00

* Soma+: 27913.84

* Soma-: -254836.89

* Contador: 241

TUBO (12): 24 Nó inicial (9): 100 Nó final (8) : 90 Compr.(ft): 6000.00 Diam.(in): 10.00

* Soma+: 546463.06

* Soma-: -14553.72

* Contador: 241

TUBO (13): 26 Nó inicial (14): 150 Nó final (9) : 100 Compr.(ft): 6000.00 Diam.(in): 12.00

* Soma+: $\quad 0.00$

* Soma-: -725017.00

* Contador: 241

TUBO (14): 28 Nó inicial (7): 80 Nó final (14) : 150 Compr.(ft): 6000.00 Diam.(in): 10.00

* Soma+: 279434.81

* Soma-: $\quad-8471.83$

* Contador: 241

TUBO (15): 30 Nó inicial (2): 30 Nó final (5) : 60 Compr.(ft): 6000.00 Diam.(in): 10.00

* Soma+: 1731744.62

* Soma-: $\quad 0.00$

* Contador: 241 
Estudo da Localização Otimizada de Equipamentos em Rede de Distribuição de Água para Detecção de Contaminação

TUBO (16): 32 Nó inicial (2): 30 Nó final (3) : 40 Compr.(ft): 6000.00 Diam.(in): 10.00

* Soma+: 1532301.75

* Soma-: 0.00

* Contador: 241

TUBO (17): 34 Nó inicial (2): 30 Nó final (4) : 50 Compr.(ft): 9000.00 Diam.(in): 10.00

* Soma+: 1430503.12

* Soma-: 0.00

* Contador: 241

TUBO (18): 36 Nó inicial (3): 40 Nó final (4) : 50 Compr.(ft): 6000.00 Diam.(in): 10.00

* Soma+: 824305.81

* Soma-: 0.00

* Contador: 241

TUBO (19): 38 Nó inicial (4): 50 Nó final (7) : 80 Compr.(ft): 6000.00 Diam.(in): 10.00

* Soma+: 1050752.50

* Soma-: 0.00

* Contador: 241

TUBO (20): 40 Nó inicial (13): 140 Nó final (7) : 80 Compr.(ft): 6000.00 Diam.(in): 10.00

* Soma+: $\quad 0.00$

* Soma-: -692889.38

* Contador: 241

TUBO (21): 42 Nó inicial (13): 140 Nó final (14) : 150 Compr.(ft): 6000.00 Diam.(in): 8.00

* Soma+: $\quad 0.00$

* Soma-: -536864.81

* Contador: 241

TUBO (22): 44 Nó inicial (14): 150 Nó final (15) : 160 Compr.(ft): 6000.00 Diam.(in): 8.00

* Soma+: 826505.00

* Soma-: -60379.48

* Contador: 241

TUBO (23): 46 Nó inicial (15): 160 Nó final (9) : 100 Compr.(ft): 6000.00 Diam.(in): 8.00

* Soma+: 18600.40

* Soma-: -1042251.75

* Contador: 241

TUBO (24): 48 Nó inicial (10): 110 Nó final (9) : 100 Compr.(ft): 6000.00 Diam.(in): 8.00

* Soma+: 1055033.00

* Soma-: $\quad 0.00$

* Contador: 241

TUBO (25): 50 Nó inicial (10): 110 Nó final (15) : 160 Compr.(ft): 6000.00 Diam.(in): 10.00

* Soma+: 1833835.12 
Estudo da Localização Otimizada de Equipamentos em Rede de Distribuição de Água para Deteç̧ão de Contaminação

* Soma-: $\quad 0.00$

* Contador: 241

TUBO (26): 52 Nó inicial (11): 120 Nó final (10) : 110 Compr.(ft): 6000.00 Diam.(in): 8.00

* Soma+: $\quad 0.00$

* Soma-: -1640359.12

* Contador: 241

TUBO (27): 56 Nó inicial (12): 130 Nó final (11) : 120 Compr.(ft): 6000.00 Diam.(in): 8.00

* Soma+: $\quad 0.00$

* Soma-: -534114.38

* Contador: 241

TUBO (28): 58 Nó inicial (12): 130 Nó final (15) : 160 Compr.(ft): 6000.00 Diam.(in): 10.00

* Soma+: 63318.39

* Soma-: -646605.50

* Contador: 241

TUBO (29): 60 Nó inicial (16): 170 Nó final (12) : 130 Compr.(ft): 6000.00 Diam.(in): 8.00

* Soma+: 43074.71

* Soma-: -382330.75

* Contador: 241

TUBO (30): 62 Nó inicial (13): 140 Nó final (15) : 160 Compr.(ft): 6000.00 Diam.(in): 8.00

* Soma+: 639524.62

* Soma-: -118161.22

* Contador: 241

TUBO (31): 64 Nó inicial (13): 140 Nó final (16) : 170 Compr.(ft): 12000.00 Diam.(in): 8.00

* Soma+: 766988.38

* Soma-: 0.00

* Contador: 241

TUBO (32): 66 Nó inicial (4): 50 Nó final (13) : 140 Compr.(ft): 12000.00 Diam.(in): 8.00

* Soma+: 775092.19

* Soma-: 0.00

* Contador: 241

TUBO (33): 78 Nó inicial (18): 65 Nó final (5) : 60 Compr.(ft): 100.00 Diam.(in): 12.00

* Soma+: 525809.12

* Soma-: -574953.56

* Contador: 217

TUBO (34): 80 Nó inicial (15): 160 Nó final (19) : 165 Compr.(ft): 100.00 Diam.(in): 12.00

* Soma+: 697376.44

* Soma-: -768424.44

* Contador: 185 
Estudo da Localização Otimizada de Equipamentos em Rede de Distribuição de Água para Deteç̧ão de Contaminação

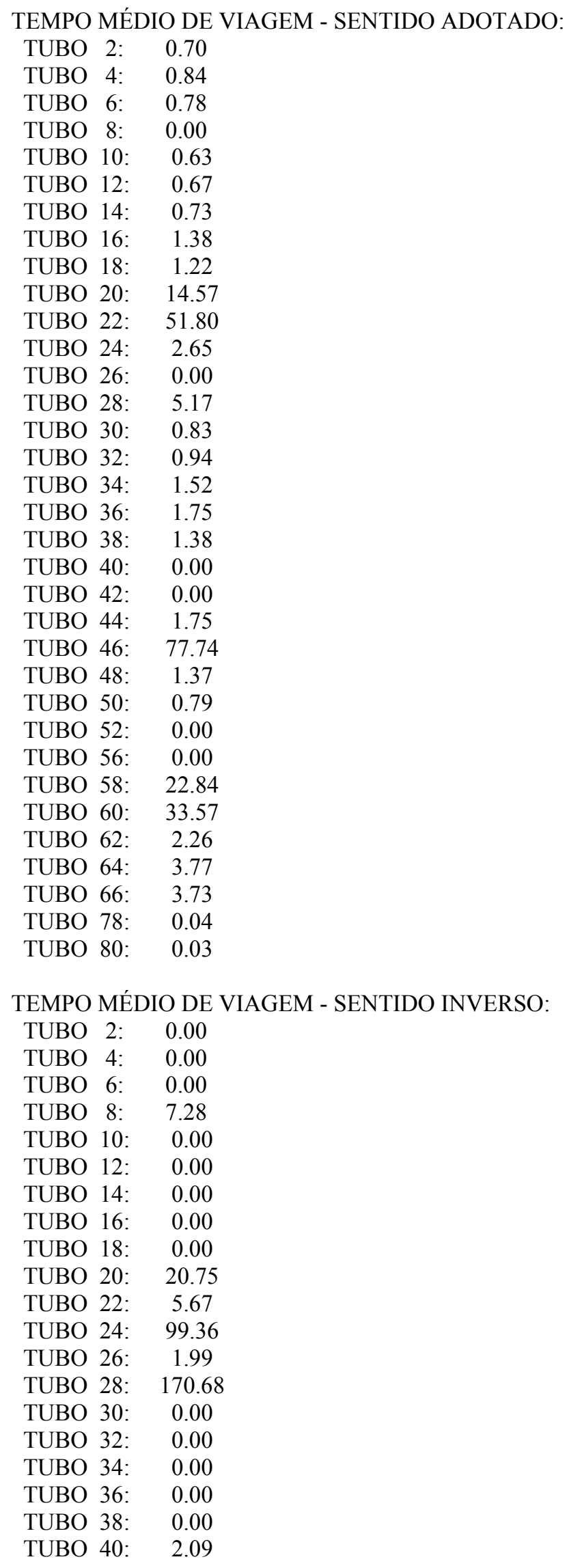


Estudo da Localização Otimizada de Equipamentos em Rede de Distribuição de Água para Deteç̧ão de Contaminação

TUBO 42: $\quad 2.69$

TUBO 44: 23.95

TUBO 46: 1.39

TUBO 48: $\quad 0.00$

TUBO 50: $\quad 0.00$

TUBO 52: $\quad 0.88$

TUBO 56: 2.71

TUBO 58: $\quad 2.24$

TUBO 60: 3.78

TUBO 62: $\quad 12.24$

TUBO 64: $\quad 0.00$

TUBO 66: $\quad 0.00$

TUBO 78: $\quad 0.04$

TUBO 80: 0.02

Matriz Custo de Tempo de viagem Inicial (h):

\begin{tabular}{|c|c|c|c|c|c|c|c|c|c|c|c|c|c|c|c|c|c|c|c|}
\hline Nós & 20 & 30 & 40 & 50 & 60 & 70 & 80 & 90 & 100 & 110 & 120 & 130 & 140 & 150 & 160 & 170 & 10 & 65 & 165 \\
\hline 20 & 0 & 0.84 & 888 & 888 & 888 & 0.70 & 8888 & 888 & 888 & 0.78 & 888 & 888 & 888 & 888 & $\begin{array}{lll}8 & 88\end{array}$ & 888 & 888 & 888 & 888 \\
\hline 30 & 888 & 0 & 0.94 & 1.52 & 0.83 & 888 & 888 & 888 & 888 & 888 & 888 & 888 & 888 & 888 & 888 & 888 & 888 & 888 & 888 \\
\hline 40 & 888 & 888 & 0 & 1.75 & 888 & 888 & 888 & 888 & 888 & 888 & 888 & 888 & 888 & 888 & $\begin{array}{lll}8 & 8 & 8\end{array}$ & 888 & 888 & 888 & 888 \\
\hline 50 & 888 & 888 & 888 & 0 & 888 & 888 & 1.38 & 888 & 888 & 888 & 888 & 888 & 3.73 & 888 & 888 & 888 & 888 & 888 & 888 \\
\hline 60 & 888 & 888 & 888 & 888 & 0 & 888 & 1.22 & 1.38 & 888 & 888 & $\begin{array}{lll}88 & 88\end{array}$ & 888 & 888 & 888 & 8888 & 888 & 888 & 0.04 & 888 \\
\hline 70 & 888 & 7.28 & 888 & 888 & 0.73 & 0 & 888 & 0.67 & 0.63 & 888 & 888 & 8888 & 888 & 888 & 888 & 888 & 888 & 888 & 888 \\
\hline 80 & 888 & 888 & 888 & 888 & 888 & 888 & 0 & 14.57 & 888 & 888 & 888 & 888 & 2.09 & 5.17 & 888 & 888 & 888 & 888 & 888 \\
\hline 90 & 888 & 888 & 888 & 888 & 888 & 888 & 20.75 & 0 & 99.36 & 888 & 888 & 888 & 888 & 5.67 & 8888 & 888 & 888 & 888 & 888 \\
\hline 100 & 888 & 888 & 888 & 888 & 888 & 888 & 888 & 2.65 & 0 & 888 & 888 & 8888 & 8888 & 1.99 & 1.39 & 888 & 888 & 888 & 888 \\
\hline 110 & 888 & 888 & 888 & 888 & 888 & 888 & 888 & 888 & 1.37 & 0 & 0.88 & 888 & 888 & 888 & 0.79 & 888 & 888 & 888 & 888 \\
\hline 120 & 888 & 888 & 888 & 888 & 888 & 888 & 888 & 888 & 888 & 888 & 0 & 2.71 & 888 & 888 & $\begin{array}{llll}8 & 8 & 8\end{array}$ & 888 & 888 & 888 & 888 \\
\hline 130 & 888 & 888 & 888 & 888 & 888 & 888 & 888 & 888 & 888 & 888 & 888 & 0 & 888 & 888 & 22.84 & 3.78 & 888 & 888 & 888 \\
\hline 140 & 888 & 888 & 888 & 888 & 888 & 888 & 888 & 888 & 888 & 888 & 888 & $\begin{array}{lll}8 & 88 \\
\end{array}$ & 0 & 888 & 2.26 & 3.77 & 888 & 888 & 888 \\
\hline 150 & 888 & 888 & 888 & 888 & 888 & 888 & 170.7 & 51.80 & 888 & 888 & 888 & 888 & 2.69 & 0 & 1.75 & 888 & 888 & 888 & 888 \\
\hline 160 & 888 & 888 & 888 & 888 & 888 & 888 & 888 & 8888 & 77.74 & 888 & 888 & 2.24 & 12.24 & 23.95 & 0 & 888 & 888 & 888 & 0.03 \\
\hline 170 & 888 & 888 & 888 & 888 & 888 & 888 & 888 & 888 & 8888 & 888 & 888 & 33.57 & 8888 & 888 & 888 & 0 & 888 & 888 & 888 \\
\hline 10 & 0 & 888 & 888 & 888 & 888 & 888 & 888 & 888 & 888 & 888 & 888 & 888 & 888 & 888 & 888 & 888 & 0 & 888 & 888 \\
\hline 65 & 888 & 888 & 888 & 888 & 0.04 & 888 & 888 & 888 & 888 & 888 & $\begin{array}{llll}8 & 8 & 8\end{array}$ & $\begin{array}{llll}8 & 8 & 8\end{array}$ & $\begin{array}{llll}8 & 8 & 8\end{array}$ & 888 & 8888 & 888 & 888 & 0 & 888 \\
\hline 165 & 888 & 888 & 888 & 888 & 888 & 888 & 888 & 888 & 888 & 888 & 888 & 8888 & 8888 & 888 & 0.02 & 888 & 888 & 888 & 0 \\
\hline
\end{tabular}


Estudo da Localização Otimizada de Equipamentos em Rede de Distribuição de Água para Deteç̧ão de Contaminação

Matriz Roteamento Inicial 1:

\begin{tabular}{|c|c|c|c|c|c|c|c|c|c|c|c|c|c|c|c|c|c|c|c|}
\hline Nós & $\mathbf{2 0}$ & $\mathbf{3 0}$ & $\mathbf{4 0}$ & $\mathbf{5 0}$ & $\mathbf{6 0}$ & $\mathbf{7 0}$ & $\mathbf{8 0}$ & $\mathbf{9 0}$ & $\mathbf{1 0 0}$ & $\mathbf{1 1 0}$ & $\mathbf{1 2 0}$ & $\mathbf{1 3 0}$ & $\mathbf{1 4 0}$ & $\mathbf{1 5 0}$ & $\mathbf{1 6 0}$ & $\mathbf{1 7 0}$ & $\mathbf{1 0}$ & $\mathbf{6 5}$ & $\mathbf{1 6 5}$ \\
\hline $\mathbf{2 0}$ & 1 & 2 & 0 & 0 & 0 & 6 & 0 & 0 & 0 & $\mathbf{1 0}$ & 0 & 0 & 0 & 0 & 0 & 0 & 0 & 0 & 0 \\
\hline $\mathbf{3 0}$ & 0 & 2 & 3 & 4 & 5 & 0 & 0 & 0 & 0 & 0 & 0 & 0 & 0 & 0 & 0 & 0 & 0 & 0 & 0 \\
\hline $\mathbf{4 0}$ & 0 & 0 & 3 & 4 & 0 & 0 & 0 & 0 & 0 & 0 & 0 & 0 & 0 & 0 & 0 & 0 & 0 & 0 & 0 \\
\hline $\mathbf{5 0}$ & 0 & 0 & 0 & 4 & 0 & 0 & 7 & 0 & 0 & 0 & 0 & 0 & 13 & 0 & 0 & 0 & 0 & 0 & 0 \\
\hline $\mathbf{6 0}$ & 0 & 0 & 0 & 0 & 5 & 0 & 7 & 8 & 0 & 0 & 0 & 0 & 0 & 0 & 0 & 0 & 0 & 18 & 0 \\
\hline $\mathbf{7 0}$ & 0 & 2 & 0 & 0 & 5 & 6 & 0 & 8 & 9 & 0 & 0 & 0 & 0 & 0 & 0 & 0 & 0 & 0 & 0 \\
\hline $\mathbf{8 0}$ & 0 & 0 & 0 & 0 & 0 & 0 & 7 & 8 & 0 & 0 & 0 & 0 & 13 & 14 & 0 & 0 & 0 & 0 & 0 \\
\hline $\mathbf{9 0}$ & 0 & 0 & 0 & 0 & 0 & 0 & 7 & 8 & 9 & 0 & 0 & 0 & 0 & 14 & 0 & 0 & 0 & 0 & 0 \\
\hline $\mathbf{1 0 0}$ & 0 & 0 & 0 & 0 & 0 & 0 & 0 & 8 & 9 & 0 & 0 & 0 & 0 & 14 & 15 & 0 & 0 & 0 & 0 \\
\hline $\mathbf{1 1 0}$ & 0 & 0 & 0 & 0 & 0 & 0 & 0 & 0 & 9 & 10 & 11 & 0 & 0 & 0 & 15 & 0 & 0 & 0 & 0 \\
\hline $\mathbf{1 2 0}$ & 0 & 0 & 0 & 0 & 0 & 0 & 0 & 0 & 0 & 0 & 11 & 12 & 0 & 0 & 0 & 0 & 0 & 0 & 0 \\
\hline $\mathbf{1 3 0}$ & 0 & 0 & 0 & 0 & 0 & 0 & 0 & 0 & 0 & 0 & 0 & 12 & 0 & 0 & 15 & 16 & 0 & 0 & 0 \\
\hline $\mathbf{1 4 0}$ & 0 & 0 & 0 & 0 & 0 & 0 & 0 & 0 & 0 & 0 & 0 & 0 & 13 & 0 & 15 & 16 & 0 & 0 & 0 \\
\hline $\mathbf{1 5 0}$ & 0 & 0 & 0 & 0 & 0 & 0 & 7 & 8 & 0 & 0 & 0 & 0 & 13 & 14 & 15 & 0 & 0 & 0 & 0 \\
\hline $\mathbf{1 6 0}$ & 0 & 0 & 0 & 0 & 0 & 0 & 0 & 0 & 9 & 0 & 0 & 12 & 13 & 14 & 15 & 0 & 0 & 0 & 19 \\
\hline $\mathbf{1 7 0}$ & 0 & 0 & 0 & 0 & 0 & 0 & 0 & 0 & 0 & 0 & 0 & 12 & 0 & 0 & 0 & 16 & 0 & 0 & 0 \\
\hline $\mathbf{1 0}$ & 1 & 0 & 0 & 0 & 0 & 0 & 0 & 0 & 0 & 0 & 0 & 0 & 0 & 0 & 0 & 0 & 17 & 0 & 0 \\
\hline $\mathbf{6 5}$ & 0 & 0 & 0 & 0 & 5 & 0 & 0 & 0 & 0 & 0 & 0 & 0 & 0 & 0 & 0 & 0 & 0 & 18 & 0 \\
\hline $\mathbf{1 6 5}$ & 0 & 0 & 0 & 0 & 0 & 0 & 0 & 0 & 0 & 0 & 0 & 0 & 0 & 0 & 15 & 0 & 0 & 0 & 19 \\
\hline
\end{tabular}

Matriz Custo de Tempo de viagem Final (h) (Caminhos Mais Curtos):

\begin{tabular}{|c|c|c|c|c|c|c|c|c|c|c|c|c|c|c|c|c|c|c|c|}
\hline Nós & 20 & 30 & 40 & 50 & 60 & 70 & 80 & 90 & 100 & 110 & 120 & 130 & 140 & 150 & 160 & 170 & 10 & 65 & 165 \\
\hline 20 & 0 & .84 & .78 & .35 & 1.43 & 0.70 & 2.64 & 1.37 & 1.33 & 0.78 & 1.66 & 3.81 & 4.73 & 3.32 & 1.57 & 7.59 & 888 & 1.47 & 1.60 \\
\hline 30 & 388 & 0 & 0.94 & 1.52 & 0.83 & 388 & 2.05 & .22 & 84.14 & 888 & 888 & 8.64 & 4.14 & 7.23 & 6.40 & 7.91 & 888 & 0.87 & 6.43 \\
\hline 40 & 888 & 888 & 0 & 1.75 & 888 & 888 & 3.13 & 17.71 & 85.22 & 888 & 888 & 9.71 & 5.22 & 8.31 & 7.48 & 8.99 & 888 & 888 & 7.50 \\
\hline 50 & 888 & 888 & 888 & 0 & 888 & 888 & 1.38 & 15.95 & 83.46 & 888 & 888 & 7.96 & 3.46 & 6.55 & 5.72 & 7.23 & 888 & 888 & 5.75 \\
\hline 60 & 888 & 888 & 888 & 888 & 0 & 888 & 1.22 & 1.38 & 83.30 & 888 & 888 & 7.80 & 3.30 & 6.39 & 5.56 & 7.07 & 888 & 0.04 & 5.59 \\
\hline 70 & 888 & 7.28 & 8.22 & 8.79 & 0.73 & 0 & 1.94 & 0.67 & 0.63 & 888 & 888 & 4.25 & 4.03 & 2.62 & 2.01 & 7.80 & 888 & 0.76 & 2.04 \\
\hline 80 & 888 & 888 & 888 & 888 & 888 & 888 & 0 & 14.57 & 82.09 & 888 & 888 & 6.58 & 2.09 & 5.17 & 4.35 & 5.86 & 888 & 888 & 4.37 \\
\hline 90 & 888 & 888 & 888 & 888 & 888 & 888 & 20.75 & 0 & 85.16 & 888 & 888 & 9.66 & 8.37 & 5.67 & 7.42 & 12.14 & 888 & 888 & 7.45 \\
\hline 100 & 888 & 888 & 888 & 888 & 888 & 888 & 23.39 & 2.65 & 0 & 888 & 888 & 3.62 & 4.69 & 1.99 & 1.39 & 7.41 & 888 & 888 & 1.41 \\
\hline 110 & 888 & 888 & 888 & 888 & 888 & 888 & 24.76 & 4.02 & 1.37 & 0 & 0.88 & 3.02 & 6.06 & 3.37 & 0.79 & 6.81 & 888 & 888 & 0.82 \\
\hline 120 & 888 & 888 & 888 & 888 & 888 & 888 & 122.0 & 101.3 & 103.3 & 000 & 0 & 2.71 & 37.78 & 49.49 & 25.54 & 6.49 & 888 & 888 & 25.57 \\
\hline 130 & 888 & 888 & 888 & 888 & 888 & 888 & 119.33 & & 100.58 & & 888 & 0 & 35.07 & 46.79 & 22.84 & 3.78 & 888 & 888 & 22.86 \\
\hline 140 & 888 & 888 & 888 & 888 & 888 & 888 & 98.76 & 78.01 & 80 & 888 & 888 & 4.50 & 0 & 26.21 & 2.26 & 3.77 & 888 & 888 & 2.29 \\
\hline 150 & 888 & 888 & 888 & 888 & 888 & 888 & 72.55 & 51.80 & 79.49 & 888 & 888 & 3.99 & 2.69 & 0 & 1.75 & 6.46 & 888 & 888 & 1.78 \\
\hline 160 & 888 & 888 & 888 & 888 & 888 & 888 & 96.50 & 75.75 & 77.74 & 888 & 888 & 2.24 & 12.24 & 23.95 & 0 & 6.02 & 888 & 888 & 0.03 \\
\hline 170 & 888 & 888 & 888 & 888 & 888 & 888 & 152.9 & 132.2 & 134.1 & 888 & 888 & 33.57 & 68.64 & 80.36 & 56.41 & 0 & 888 & 888 & 56.43 \\
\hline 10 & 0 & 0.84 & 1.78 & 2.35 & 1.43 & 0.70 & 2.64 & 1.37 & 1.33 & 0.78 & 1.66 & 3.81 & 4.73 & 3.32 & 1.57 & 7.59 & 0 & 1.47 & 1.60 \\
\hline 65 & 888 & 888 & 888 & 888 & 0.04 & 888 & 1.26 & 1.42 & 83.35 & 888 & 888 & 7.84 & 3.34 & 6.43 & 5.61 & 7.11 & 888 & 0 & 5.63 \\
\hline 165 & 888 & 888 & 888 & 888 & 888 & 888 & 96.52 & 75.77 & 77.76 & 888 & 888 & 2.26 & 12.26 & 23.97 & 0.02 & 6.04 & 888 & 888 & 0 \\
\hline
\end{tabular}


Estudo da Localização Otimizada de Equipamentos em Rede de Distribuição de Água para Deteç̧ão de Contaminação

Matriz Roteamento Final:

\begin{tabular}{|c|c|c|c|c|c|c|c|c|c|c|c|c|c|c|c|c|c|c|c|}
\hline Nós & $\mathbf{2 0}$ & $\mathbf{3 0}$ & $\mathbf{4 0}$ & $\mathbf{5 0}$ & $\mathbf{6 0}$ & $\mathbf{7 0}$ & $\mathbf{8 0}$ & $\mathbf{9 0}$ & $\mathbf{1 0 0}$ & $\mathbf{1 1 0}$ & $\mathbf{1 2 0}$ & $\mathbf{1 3 0}$ & $\mathbf{1 4 0}$ & $\mathbf{1 5 0}$ & $\mathbf{1 6 0}$ & $\mathbf{1 7 0}$ & $\mathbf{1 0}$ & $\mathbf{6 5}$ & $\mathbf{1 6 5}$ \\
\hline $\mathbf{2 0}$ & 1 & 2 & 2 & 2 & 6 & 6 & 6 & 6 & 6 & $\mathbf{1 0}$ & 10 & 10 & 6 & 6 & 10 & 10 & 0 & 6 & 10 \\
\hline $\mathbf{3 0}$ & 0 & 2 & 3 & 4 & 5 & 0 & 5 & 5 & 5 & 0 & 0 & 5 & 5 & 5 & 5 & 5 & 0 & 5 & 5 \\
\hline $\mathbf{4 0}$ & 0 & 0 & 3 & 4 & 0 & 0 & 4 & 4 & 4 & 0 & 0 & 4 & 4 & 4 & 4 & 4 & 0 & 0 & 4 \\
\hline $\mathbf{5 0}$ & 0 & 0 & 0 & 4 & 0 & 0 & 7 & 7 & 7 & 0 & 0 & 7 & 7 & 7 & 7 & 7 & 0 & 0 & 7 \\
\hline $\mathbf{6 0}$ & 0 & 0 & 0 & 0 & 5 & 0 & 7 & 8 & 7 & 0 & 0 & 7 & 7 & 7 & 7 & 7 & 0 & 18 & 7 \\
\hline $\mathbf{7 0}$ & 0 & 2 & 2 & 2 & 5 & 6 & 5 & 8 & 9 & 0 & 0 & 9 & 5 & 9 & 9 & 5 & 0 & 5 & 9 \\
\hline $\mathbf{8 0}$ & 0 & 0 & 0 & 0 & 0 & 0 & 7 & 8 & 13 & 0 & 0 & 13 & 13 & 14 & 13 & 13 & 0 & 0 & 13 \\
\hline $\mathbf{9 0}$ & 0 & 0 & 0 & 0 & 0 & 0 & 7 & 8 & 14 & 0 & 0 & 14 & 14 & 14 & 14 & 14 & 0 & 0 & 14 \\
\hline $\mathbf{1 0 0}$ & 0 & 0 & 0 & 0 & 0 & 0 & 8 & 8 & 9 & 0 & 0 & 15 & 14 & 14 & 15 & 15 & 0 & 0 & 15 \\
\hline $\mathbf{1 1 0}$ & 0 & 0 & 0 & 0 & 0 & 0 & 9 & 9 & 9 & 10 & 11 & 15 & 9 & 9 & 15 & 15 & 0 & 0 & 15 \\
\hline $\mathbf{1 2 0}$ & 0 & 0 & 0 & 0 & 0 & 0 & 12 & 12 & 12 & 0 & 11 & 12 & 12 & 12 & 12 & 12 & 0 & 0 & 12 \\
\hline $\mathbf{1 3 0}$ & 0 & 0 & 0 & 0 & 0 & 0 & 15 & 15 & 15 & 0 & 0 & 12 & 15 & 15 & 15 & 16 & 0 & 0 & 15 \\
\hline $\mathbf{1 4 0}$ & 0 & 0 & 0 & 0 & 0 & 0 & 15 & 15 & 15 & 0 & 0 & 15 & 13 & 15 & 15 & 16 & 0 & 0 & 15 \\
\hline $\mathbf{1 5 0}$ & 0 & 0 & 0 & 0 & 0 & 0 & 8 & 8 & 15 & 0 & 0 & 15 & 13 & 14 & 15 & 13 & 0 & 0 & 15 \\
\hline $\mathbf{1 6 0}$ & 0 & 0 & 0 & 0 & 0 & 0 & 14 & 14 & 9 & 0 & 0 & 12 & 13 & 14 & 15 & 12 & 0 & 0 & 19 \\
\hline $\mathbf{1 7 0}$ & 0 & 0 & 0 & 0 & 0 & 0 & 12 & 12 & 12 & 0 & 0 & 12 & 12 & 12 & 12 & 16 & 0 & 0 & 12 \\
\hline $\mathbf{1 0}$ & 1 & 1 & 1 & 1 & 1 & 1 & 1 & 1 & 1 & 1 & 1 & 1 & 1 & 1 & 1 & 1 & 17 & 1 & 1 \\
\hline $\mathbf{6 5}$ & 0 & 0 & 0 & 0 & 5 & 0 & 5 & 5 & 5 & 0 & 0 & 5 & 5 & 5 & 5 & 5 & 0 & 18 & 5 \\
\hline $\mathbf{1 6 5}$ & 0 & 0 & 0 & 0 & 0 & 0 & 15 & 15 & 15 & 0 & 0 & 15 & 15 & 15 & 15 & 15 & 0 & 0 & 19 \\
\hline
\end{tabular}

Matriz Custo de Extensão de viagem Inicial (ft):

\begin{tabular}{|c|c|c|c|c|c|c|c|c|c|c|c|c|c|c|c|c|c|c|c|}
\hline Nós & 20 & 30 & 40 & 50 & 60 & 70 & 80 & 90 & 100 & 110 & 120 & 130 & 140 & 150 & 160 & 170 & 10 & 65 & 165 \\
\hline 20 & 0 & 12000 & 228200 & 228200 & 228200 & 12000 & 228200 & 228200 & 228200 & 12000 & 228200 & 228200 & 228200 & 228200 & 228200 & 228200 & 228200 & 228200 & 228200 \\
\hline 30 & 228200 & 0 & 6000 & 9000 & 6000 & 228200 & 228200 & 228200 & 228200 & 228200 & 228200 & 228200 & 228200 & 228200 & 228200 & 228200 & 228200 & 228200 & 228200 \\
\hline 40 & 228200 & 228200 & 0 & 6000 & 228200 & 228200 & 228200 & 228200 & 228200 & 228200 & 228200 & 228200 & 228200 & 228200 & 228200 & 228200 & 228200 & 228200 & 228200 \\
\hline 50 & 228200 & 228200 & 228200 & 0 & 228200 & 228200 & 6000 & 228200 & 228200 & 228200 & 228200 & 228200 & 12000 & 228200 & 228200 & 228200 & 228200 & 228200 & 228200 \\
\hline 60 & 228200 & 228200 & 228200 & 228200 & 0 & 228200 & 60000 & 6000 & 228200 & 228200 & 228200 & 228200 & 228200 & 228200 & 228200 & 228200 & 228200 & $\left.\begin{array}{lll}1 & 0 & 0\end{array}\right]$ & 228200 \\
\hline 70 & 228200 & 90000 & 228200 & 228200 & 6000 & 0 & 228200 & 6000 & 6000 & 228200 & 228200 & 228200 & 228200 & 228200 & 228200 & 228200 & 228200 & 228200 & 228200 \\
\hline 80 & 228200 & 228200 & 228200 & 228200 & 228200 & 228200 & 0 & 6000 & 228200 & 228200 & 228200 & 228200 & 6000 & 6000 & 228200 & 228200 & 228200 & 228200 & 228200 \\
\hline 90 & 228200 & 228200 & 228200 & 228200 & 228200 & 228200 & 6000 & 0 & 60000 & 228200 & 228200 & 228200 & 228200 & 6000 & 228200 & 228200 & 228200 & 228200 & 228200 \\
\hline 100 & 228200 & 228200 & 228200 & 228200 & 228200 & 228200 & 228200 & 6000 & 0 & 228200 & 228200 & 228200 & 228200 & 6000 & 6000 & 228200 & 228200 & 228200 & 228200 \\
\hline 110 & 228200 & 228200 & 228200 & 228200 & 228200 & 228200 & 228200 & 228200 & 6000 & 0 & 6000 & 228200 & 228200 & 228200 & 6000 & 228200 & 228200 & 228200 & 228200 \\
\hline 120 & 228200 & 228200 & 228200 & 228200 & 228200 & 228200 & 228200 & 228200 & 228200 & 228200 & 0 & 60000 & 228200 & 228200 & 228200 & 228200 & 228200 & 228200 & 228200 \\
\hline 130 & 228200 & 228200 & 228200 & 228200 & 228200 & 228200 & 228200 & 228200 & 228200 & 228200 & 228200 & 0 & 228200 & 228200 & 6000 & 6000 & 228200 & 228200 & 228200 \\
\hline 140 & 228200 & 228200 & 228200 & 228200 & 228200 & 228200 & 228200 & 228200 & 228200 & 228200 & 228200 & 228200 & 0 & 228200 & 6000 & 12000 & 228200 & 228200 & 228200 \\
\hline 150 & 228200 & 228200 & 228200 & 228200 & 228200 & 228200 & 6000 & 6000 & 228200 & 228200 & 228200 & 228200 & 6000 & 0 & 60000 & 228200 & 228200 & 228200 & 228200 \\
\hline 160 & 228200 & 228200 & 228200 & 228200 & 228200 & 228200 & 228200 & 228200 & 6000 & 228200 & 228200 & 6000 & 6000 & 6000 & 0 & 228200 & 228200 & 228200 & $\begin{array}{lll}1 & 0 & 0\end{array}$ \\
\hline 170 & 228200 & 228200 & 228200 & 228200 & 228200 & 228200 & 228200 & 228200 & 228200 & 228200 & 228200 & 6000 & 228200 & 228200 & 228200 & 0 & 228200 & 228200 & 228200 \\
\hline 10 & 0 & 228200 & 228200 & 228200 & 228200 & 228200 & 228200 & 228200 & 228200 & 228200 & 228200 & 228200 & 228200 & 228200 & 228200 & 228200 & 0 & 228200 & 228200 \\
\hline 65 & 228200 & 228200 & 228200 & 228200 & $\begin{array}{lll}1 & 0 & 0\end{array}$ & 228200 & 228200 & 228200 & 228200 & 228200 & 228200 & 228200 & 228200 & 228200 & 228200 & 228200 & 228200 & 0 & 228200 \\
\hline 165 & 228200 & 228200 & 228200 & 228200 & 228200 & 228200 & 228200 & 228200 & 228200 & 228200 & 228200 & 228200 & 228200 & 228200 & $\begin{array}{lll}1 & 0 & 0\end{array}$ & 228200 & 228200 & 228200 & 0 \\
\hline
\end{tabular}


Estudo da Localização Otimizada de Equipamentos em Rede de Distribuição de Água para Deteç̧ão de Contaminação

Matriz Roteamento Inicial 2:

\begin{tabular}{|c|c|c|c|c|c|c|c|c|c|c|c|c|c|c|c|c|c|c|c|}
\hline Nós & $\mathbf{2 0}$ & $\mathbf{3 0}$ & $\mathbf{4 0}$ & $\mathbf{5 0}$ & $\mathbf{6 0}$ & $\mathbf{7 0}$ & $\mathbf{8 0}$ & $\mathbf{9 0}$ & $\mathbf{1 0 0}$ & $\mathbf{1 1 0}$ & $\mathbf{1 2 0}$ & $\mathbf{1 3 0}$ & $\mathbf{1 4 0}$ & $\mathbf{1 5 0}$ & $\mathbf{1 6 0}$ & $\mathbf{1 7 0}$ & $\mathbf{1 0}$ & $\mathbf{6 5}$ & $\mathbf{1 6 5}$ \\
\hline $\mathbf{2 0}$ & 1 & 2 & 0 & 0 & 0 & 6 & 0 & 0 & 0 & $\mathbf{1 0}$ & 0 & 0 & 0 & 0 & 0 & 0 & 0 & 0 & 0 \\
\hline $\mathbf{3 0}$ & 0 & 2 & 3 & 4 & 5 & 0 & 0 & 0 & 0 & 0 & 0 & 0 & 0 & 0 & 0 & 0 & 0 & 0 & 0 \\
\hline $\mathbf{4 0}$ & 0 & 0 & 3 & 4 & 0 & 0 & 0 & 0 & 0 & 0 & 0 & 0 & 0 & 0 & 0 & 0 & 0 & 0 & 0 \\
\hline $\mathbf{5 0}$ & 0 & 0 & 0 & 4 & 0 & 0 & 7 & 0 & 0 & 0 & 0 & 0 & 13 & 0 & 0 & 0 & 0 & 0 & 0 \\
\hline $\mathbf{6 0}$ & 0 & 0 & 0 & 0 & 5 & 0 & 7 & 8 & 0 & 0 & 0 & 0 & 0 & 0 & 0 & 0 & 0 & 18 & 0 \\
\hline $\mathbf{7 0}$ & 0 & 2 & 0 & 0 & 5 & 6 & 0 & 8 & 9 & 0 & 0 & 0 & 0 & 0 & 0 & 0 & 0 & 0 & 0 \\
\hline $\mathbf{8 0}$ & 0 & 0 & 0 & 0 & 0 & 0 & 7 & 8 & 0 & 0 & 0 & 0 & 13 & 14 & 0 & 0 & 0 & 0 & 0 \\
\hline $\mathbf{9 0}$ & 0 & 0 & 0 & 0 & 0 & 0 & 7 & 8 & 9 & 0 & 0 & 0 & 0 & 14 & 0 & 0 & 0 & 0 & 0 \\
\hline $\mathbf{1 0 0}$ & 0 & 0 & 0 & 0 & 0 & 0 & 0 & 8 & 9 & 0 & 0 & 0 & 0 & 14 & 15 & 0 & 0 & 0 & 0 \\
\hline $\mathbf{1 1 0}$ & 0 & 0 & 0 & 0 & 0 & 0 & 0 & 0 & 9 & 10 & 11 & 0 & 0 & 0 & 15 & 0 & 0 & 0 & 0 \\
\hline $\mathbf{1 2 0}$ & 0 & 0 & 0 & 0 & 0 & 0 & 0 & 0 & 0 & 0 & 11 & 12 & 0 & 0 & 0 & 0 & 0 & 0 & 0 \\
\hline $\mathbf{1 3 0}$ & 0 & 0 & 0 & 0 & 0 & 0 & 0 & 0 & 0 & 0 & 0 & 12 & 0 & 0 & 15 & 16 & 0 & 0 & 0 \\
\hline $\mathbf{1 4 0}$ & 0 & 0 & 0 & 0 & 0 & 0 & 0 & 0 & 0 & 0 & 0 & 0 & 13 & 0 & 15 & 16 & 0 & 0 & 0 \\
\hline $\mathbf{1 5 0}$ & 0 & 0 & 0 & 0 & 0 & 0 & 7 & 8 & 0 & 0 & 0 & 0 & 13 & 14 & 15 & 0 & 0 & 0 & 0 \\
\hline $\mathbf{1 6 0}$ & 0 & 0 & 0 & 0 & 0 & 0 & 0 & 0 & 9 & 0 & 0 & 12 & 13 & 14 & 15 & 0 & 0 & 0 & 19 \\
\hline $\mathbf{1 7 0}$ & 0 & 0 & 0 & 0 & 0 & 0 & 0 & 0 & 0 & 0 & 0 & 12 & 0 & 0 & 0 & 16 & 0 & 0 & 0 \\
\hline $\mathbf{1 0}$ & 1 & 0 & 0 & 0 & 0 & 0 & 0 & 0 & 0 & 0 & 0 & 0 & 0 & 0 & 0 & 0 & 17 & 0 & 0 \\
\hline $\mathbf{6 5}$ & 0 & 0 & 0 & 0 & 5 & 0 & 0 & 0 & 0 & 0 & 0 & 0 & 0 & 0 & 0 & 0 & 0 & 18 & 0 \\
\hline $\mathbf{1 6 5}$ & 0 & 0 & 0 & 0 & 0 & 0 & 0 & 0 & 0 & 0 & 0 & 0 & 0 & 0 & 15 & 0 & 0 & 0 & 19 \\
\hline
\end{tabular}

Matriz Custo de Extensão de viagem Final (ft) (Caminhos Mais Curtos):

\begin{tabular}{|c|c|c|c|c|c|c|c|c|c|c|c|c|c|c|c|c|c|c|c|}
\hline Nós & 20 & 30 & 40 & 50 & 60 & 70 & 80 & 90 & 100 & 110 & 120 & 130 & 140 & 150 & 160 & 170 & 10 & 65 & 165 \\
\hline 20 & 0 & 2000 & 18000 & 21000 & 18000 & 12000 & 24000 & 18000 & 18000 & 12000 & 18000 & 24000 & 24000 & 24000 & 18000 & 30000 & 228200 & 18100 & 18100 \\
\hline 30 & 28200 & 0 & 6000 & 90000 & 6000 & 228200 & 12000 & 12000 & 18000 & 228200 & 228200 & 30000 & 18000 & 18000 & 24000 & 30000 & 228200 & 6100 & 24100 \\
\hline 40 & 228200 & 228200 & 0 & 6000 & 228200 & 228200 & 12000 & 18000 & 24000 & 228200 & 228200 & 30000 & 18000 & 18000 & 24000 & 30000 & 228200 & 228200 & 24100 \\
\hline 50 & 8200 & 228200 & 228200 & 0 & 228200 & 228200 & 6000 & 12000 & 18000 & 228200 & 228200 & 24000 & 12000 & 12000 & 18000 & 24000 & 228200 & 228200 & 18100 \\
\hline 60 & 228200 & 228200 & 228200 & 228200 & 0 & 228200 & 6000 & 6000 & 12000 & 228200 & 228200 & 24000 & 12000 & 12000 & 18000 & 24000 & 228200 & $\begin{array}{lll}1 & 0 & 0\end{array}$ & 18100 \\
\hline 70 & 228200 & 9000 & 15000 & 18000 & 6000 & 0 & 12000 & 6000 & 6000 & 228200 & 228200 & 18000 & 18000 & 12000 & 12000 & 24000 & 228200 & 6100 & 12100 \\
\hline 80 & 228200 & 228200 & 228200 & 228200 & 228200 & 228200 & 0 & 6000 & 12000 & 228200 & 228200 & 18000 & 6000 & 6000 & 12000 & 18000 & 228200 & 228200 & 12100 \\
\hline 90 & 228200 & 228200 & 228200 & 228200 & 228200 & 228200 & 6000 & 0 & 6000 & 228200 & 228200 & 18000 & 12000 & 6000 & 12000 & 24000 & 228200 & 228200 & 12100 \\
\hline 100 & 228200 & 228200 & 228200 & 228200 & 228200 & 228200 & 12000 & 60000 & 0 & 228200 & 228200 & 12000 & 12000 & 6000 & 6000 & 18000 & 228200 & 228200 & 6100 \\
\hline 110 & 228200 & 228200 & 228200 & 228200 & 228200 & 228200 & 18000 & 12000 & 6000 & 0 & 6000 & 12000 & 12000 & 12000 & 6000 & 18000 & 228200 & 228200 & 6100 \\
\hline 120 & 228200 & 228200 & 228200 & 228200 & 228200 & 228200 & 24000 & 24000 & 18000 & 228200 & 0 & 6000 & 18000 & 18000 & 12000 & 12000 & 228200 & 228200 & 12100 \\
\hline 130 & 228200 & 228200 & 228200 & 228200 & 228200 & 228200 & 18000 & 18000 & 12000 & 228200 & 228200 & 0 & 12000 & 12000 & 6000 & 6000 & 228200 & 228200 & 6100 \\
\hline 140 & 228200 & 228200 & 228200 & 228200 & 228200 & 228200 & 18000 & 18000 & 12000 & 228200 & 228200 & 12000 & 0 & 12000 & 6000 & 12000 & 228200 & 228200 & 6100 \\
\hline 150 & 228200 & 228200 & 228200 & 228200 & 228200 & 228200 & 6000 & 6000 & 12000 & 228200 & 228200 & 12000 & 6000 & 0 & 6000 & 18000 & 228200 & 228200 & 6100 \\
\hline 160 & 228200 & 228200 & 228200 & 228200 & 228200 & 228200 & 12000 & 12000 & 6000 & 228200 & 228200 & 6000 & 6000 & 6000 & 0 & 12000 & 228200 & 228200 & $\begin{array}{lll}1 & 0 & 0\end{array}$ \\
\hline 170 & 228200 & 228200 & 228200 & 228200 & 228200 & 228200 & 24000 & 24000 & 18000 & 228200 & 228200 & 6000 & 18000 & 18000 & 12000 & 0 & 228200 & 228200 & 12100 \\
\hline 10 & 0 & 12000 & 18000 & 21000 & 18000 & 12000 & 24000 & 18000 & 18000 & 12000 & 18000 & 24000 & 24000 & 24000 & 18000 & 30000 & 0 & 18100 & 18100 \\
\hline 65 & 228200 & 228200 & 228200 & 228200 & $\begin{array}{lll}1 & 0 & 0\end{array}$ & 228200 & 6100 & 6100 & 12100 & 228200 & 228200 & 24100 & 12100 & 12100 & 18100 & 24100 & 228200 & 0 & 18200 \\
\hline 165 & 228200 & 228200 & 228200 & 228200 & 228200 & 228200 & 12100 & 12100 & 6100 & 228200 & 228200 & 6100 & 6100 & 6100 & 100 & 12100 & 228200 & 228200 & 0 \\
\hline
\end{tabular}


Estudo da Localização Otimizada de Equipamentos em Rede de Distribuição de Água para Deteç̧ão de Contaminação

Matriz Roteamento Final 2:

\begin{tabular}{|c|c|c|c|c|c|c|c|c|c|c|c|c|c|c|c|c|c|c|c|}
\hline Nós & $\mathbf{2 0}$ & $\mathbf{3 0}$ & $\mathbf{4 0}$ & $\mathbf{5 0}$ & $\mathbf{6 0}$ & $\mathbf{7 0}$ & $\mathbf{8 0}$ & $\mathbf{9 0}$ & $\mathbf{1 0 0}$ & $\mathbf{1 1 0}$ & $\mathbf{1 2 0}$ & $\mathbf{1 3 0}$ & $\mathbf{1 4 0}$ & $\mathbf{1 5 0}$ & $\mathbf{1 6 0}$ & $\mathbf{1 7 0}$ & $\mathbf{1 0}$ & $\mathbf{6 5}$ & $\mathbf{1 6 5}$ \\
\hline $\mathbf{2 0}$ & 1 & 2 & 2 & 2 & 2 & 6 & 2 & 6 & 6 & $\mathbf{1 0}$ & 10 & 10 & 10 & 6 & 10 & 10 & 0 & 2 & 10 \\
\hline $\mathbf{3 0}$ & 0 & 2 & 3 & 4 & 5 & 0 & 5 & 5 & 5 & 0 & 0 & 5 & 5 & 5 & 5 & 5 & 0 & 5 & 5 \\
\hline $\mathbf{4 0}$ & 0 & 0 & 3 & 4 & 0 & 0 & 4 & 4 & 4 & 0 & 0 & 4 & 4 & 4 & 4 & 4 & 0 & 0 & 4 \\
\hline $\mathbf{5 0}$ & 0 & 0 & 0 & 4 & 0 & 0 & 7 & 7 & 7 & 0 & 0 & 13 & 13 & 7 & 13 & 13 & 0 & 0 & 13 \\
\hline $\mathbf{6 0}$ & 0 & 0 & 0 & 0 & 5 & 0 & 7 & 8 & 8 & 0 & 0 & 8 & 7 & 7 & 8 & 7 & 0 & 18 & 8 \\
\hline $\mathbf{7 0}$ & 0 & 2 & 2 & 2 & 5 & 6 & 5 & 8 & 9 & 0 & 0 & 9 & 5 & 8 & 9 & 9 & 0 & 5 & 9 \\
\hline $\mathbf{8 0}$ & 0 & 0 & 0 & 0 & 0 & 0 & 7 & 8 & 8 & 0 & 0 & 13 & 13 & 14 & 13 & 13 & 0 & 0 & 13 \\
\hline $\mathbf{9 0}$ & 0 & 0 & 0 & 0 & 0 & 0 & 7 & 8 & 9 & 0 & 0 & 9 & 7 & 14 & 9 & 7 & 0 & 0 & 9 \\
\hline $\mathbf{1 0 0}$ & 0 & 0 & 0 & 0 & 0 & 0 & 8 & 8 & 9 & 0 & 0 & 15 & 14 & 14 & 15 & 15 & 0 & 0 & 15 \\
\hline $\mathbf{1 1 0}$ & 0 & 0 & 0 & 0 & 0 & 0 & 9 & 9 & 9 & 10 & 11 & 11 & 15 & 9 & 15 & 11 & 0 & 0 & 15 \\
\hline $\mathbf{1 2 0}$ & 0 & 0 & 0 & 0 & 0 & 0 & 12 & 12 & 12 & 0 & 11 & 12 & 12 & 12 & 12 & 12 & 0 & 0 & 12 \\
\hline $\mathbf{1 3 0}$ & 0 & 0 & 0 & 0 & 0 & 0 & 15 & 15 & 15 & 0 & 0 & 12 & 15 & 15 & 15 & 16 & 0 & 0 & 15 \\
\hline $\mathbf{1 4 0}$ & 0 & 0 & 0 & 0 & 0 & 0 & 15 & 15 & 15 & 0 & 0 & 15 & 13 & 15 & 15 & 16 & 0 & 0 & 15 \\
\hline $\mathbf{1 5 0}$ & 0 & 0 & 0 & 0 & 0 & 0 & 7 & 8 & 8 & 0 & 0 & 15 & 13 & 14 & 15 & 13 & 0 & 0 & 15 \\
\hline $\mathbf{1 6 0}$ & 0 & 0 & 0 & 0 & 0 & 0 & 14 & 9 & 9 & 0 & 0 & 12 & 13 & 14 & 15 & 12 & 0 & 0 & 19 \\
\hline $\mathbf{1 7 0}$ & 0 & 0 & 0 & 0 & 0 & 0 & 12 & 12 & 12 & 0 & 0 & 12 & 12 & 12 & 12 & 16 & 0 & 0 & 12 \\
\hline $\mathbf{1 0}$ & 1 & 1 & 1 & 1 & 1 & 1 & 1 & 1 & 1 & 1 & 1 & 1 & 1 & 1 & 1 & 1 & 17 & 1 & 1 \\
\hline $\mathbf{6 5}$ & 0 & 0 & 0 & 0 & 5 & 0 & 5 & 5 & 5 & 0 & 0 & 5 & 5 & 5 & 5 & 5 & 0 & 18 & 5 \\
\hline $\mathbf{1 6 5}$ & 0 & 0 & 0 & 0 & 0 & 0 & 15 & 15 & 15 & 0 & 0 & 15 & 15 & 15 & 15 & 15 & 0 & 0 & 19 \\
\hline
\end{tabular}

Matriz Poluição para Volume Consumido (nível de serviço: 468.10m3):

\begin{tabular}{|c|c|c|c|c|c|c|c|c|c|c|c|c|c|c|c|c|c|c|c|}
\hline Nós & $\mathbf{2 0}$ & $\mathbf{3 0}$ & $\mathbf{4 0}$ & $\mathbf{5 0}$ & $\mathbf{6 0}$ & $\mathbf{7 0}$ & $\mathbf{8 0}$ & $\mathbf{9 0}$ & $\mathbf{1 0 0}$ & $\mathbf{1 1 0}$ & $\mathbf{1 2 0}$ & $\mathbf{1 3 0}$ & $\mathbf{1 4 0}$ & $\mathbf{1 5 0}$ & $\mathbf{1 6 0}$ & $\mathbf{1 7 0}$ & $\mathbf{1 0}$ & $\mathbf{6 5}$ & $\mathbf{1 6 5}$ \\
\hline $\mathbf{2 0}$ & 1 & 1 & 0 & 0 & 1 & 1 & 0 & 1 & 1 & 1 & 0 & 0 & 0 & 0 & 0 & 0 & 0 & 1 & 0 \\
\hline $\mathbf{3 0}$ & 0 & 1 & 1 & 1 & 1 & 0 & 1 & 1 & 0 & 0 & 0 & 0 & 0 & 0 & 0 & 0 & 0 & 1 & 0 \\
\hline $\mathbf{4 0}$ & 0 & 0 & 1 & 1 & 0 & 0 & 1 & 0 & 0 & 0 & 0 & 0 & 0 & 0 & 0 & 0 & 0 & 0 & 0 \\
\hline $\mathbf{5 0}$ & 0 & 0 & 0 & 1 & 0 & 0 & 1 & 0 & 0 & 0 & 0 & 0 & 1 & 0 & 0 & 0 & 0 & 0 & 0 \\
\hline $\mathbf{6 0}$ & 0 & 0 & 0 & 0 & 1 & 0 & 1 & 1 & 0 & 0 & 0 & 0 & 0 & 0 & 0 & 0 & 0 & 1 & 0 \\
\hline $\mathbf{7 0}$ & 0 & 0 & 0 & 0 & 1 & 1 & 0 & 1 & 1 & 0 & 0 & 0 & 0 & 0 & 0 & 0 & 0 & 1 & 0 \\
\hline $\mathbf{8 0}$ & 0 & 0 & 0 & 0 & 0 & 0 & 1 & 0 & 0 & 0 & 0 & 0 & 1 & 0 & 0 & 0 & 0 & 0 & 0 \\
\hline $\mathbf{9 0}$ & 0 & 0 & 0 & 0 & 0 & 0 & 0 & 1 & 0 & 0 & 0 & 0 & 0 & 0 & 0 & 0 & 0 & 0 & 0 \\
\hline $\mathbf{1 0 0}$ & 0 & 0 & 0 & 0 & 0 & 0 & 0 & 0 & 1 & 0 & 0 & 0 & 0 & 1 & 1 & 0 & 0 & 0 & 1 \\
\hline $\mathbf{1 1 0}$ & 0 & 0 & 0 & 0 & 0 & 0 & 0 & 0 & 1 & 1 & 1 & 0 & 0 & 0 & 1 & 0 & 0 & 0 & 1 \\
\hline $\mathbf{1 2 0}$ & 0 & 0 & 0 & 0 & 0 & 0 & 0 & 0 & 0 & 0 & 1 & 1 & 0 & 0 & 0 & 1 & 0 & 0 & 0 \\
\hline $\mathbf{1 3 0}$ & 0 & 0 & 0 & 0 & 0 & 0 & 0 & 0 & 0 & 0 & 0 & 1 & 0 & 0 & 0 & 1 & 0 & 0 & 0 \\
\hline $\mathbf{1 4 0}$ & 0 & 0 & 0 & 0 & 0 & 0 & 0 & 0 & 0 & 0 & 0 & 0 & 1 & 0 & 1 & 1 & 0 & 0 & 1 \\
\hline $\mathbf{1 5 0}$ & 0 & 0 & 0 & 0 & 0 & 0 & 0 & 0 & 0 & 0 & 0 & 0 & 1 & 1 & 1 & 0 & 0 & 0 & 1 \\
\hline $\mathbf{1 6 0}$ & 0 & 0 & 0 & 0 & 0 & 0 & 0 & 0 & 0 & 0 & 0 & 1 & 0 & 0 & 1 & 0 & 0 & 0 & 1 \\
\hline $\mathbf{1 7 0}$ & 0 & 0 & 0 & 0 & 0 & 0 & 0 & 0 & 0 & 0 & 0 & 0 & 0 & 0 & 0 & 1 & 0 & 0 & 0 \\
\hline $\mathbf{1 0}$ & 1 & 1 & 0 & 0 & 1 & 1 & 0 & 1 & 1 & 1 & 0 & 0 & 0 & 0 & 0 & 0 & 0 & 1 & 0 \\
\hline $\mathbf{6 5}$ & 0 & 0 & 0 & 0 & 1 & 0 & 1 & 1 & 0 & 0 & 0 & 0 & 0 & 0 & 0 & 0 & 0 & 0 & 0 \\
\hline $\mathbf{1 6 5}$ & 0 & 0 & 0 & 0 & 0 & 0 & 0 & 0 & 0 & 0 & 0 & 1 & 0 & 0 & 1 & 0 & 0 & 0 & 0 \\
\hline
\end{tabular}


Estudo da Localização Otimizada de Equipamentos em Rede

de Distribuição de Água para Deteç̧ão de Contaminação

Matriz Poluição para Tempo de Detecção (nível de serviço: 6.00h.)

\begin{tabular}{|c|c|c|c|c|c|c|c|c|c|c|c|c|c|c|c|c|c|c|c|}
\hline Nós & $\mathbf{2 0}$ & $\mathbf{3 0}$ & $\mathbf{4 0}$ & $\mathbf{5 0}$ & $\mathbf{6 0}$ & $\mathbf{7 0}$ & $\mathbf{8 0}$ & $\mathbf{9 0}$ & $\mathbf{1 0 0}$ & $\mathbf{1 1 0}$ & $\mathbf{1 2 0}$ & $\mathbf{1 3 0}$ & $\mathbf{1 4 0}$ & $\mathbf{1 5 0}$ & $\mathbf{1 6 0}$ & $\mathbf{1 7 0}$ & $\mathbf{1 0}$ & $\mathbf{6 5}$ & $\mathbf{1 6 5}$ \\
\hline $\mathbf{2 0}$ & 1 & 1 & 1 & 1 & 1 & $\mathbf{1}$ & 1 & 1 & 1 & 1 & $\mathbf{1}$ & 1 & 1 & 1 & 1 & 0 & 0 & 1 & 1 \\
\hline $\mathbf{3 0}$ & 0 & 1 & 1 & 1 & 1 & 0 & 1 & 1 & 0 & 0 & 0 & 0 & 1 & 0 & 0 & 0 & 0 & 1 & 0 \\
\hline $\mathbf{4 0}$ & 0 & 0 & 1 & 1 & 0 & 0 & 1 & 0 & 0 & 0 & 0 & 0 & 1 & 0 & 0 & 0 & 0 & 0 & 0 \\
\hline $\mathbf{5 0}$ & 0 & 0 & 0 & 1 & 0 & 0 & 1 & 0 & 0 & 0 & 0 & 0 & 1 & 0 & 1 & 0 & 0 & 0 & 1 \\
\hline $\mathbf{6 0}$ & 0 & 0 & 0 & 0 & 1 & 0 & 1 & 1 & 0 & 0 & 0 & 0 & 1 & 0 & 1 & 0 & 0 & 1 & 1 \\
\hline $\mathbf{7 0}$ & 0 & 0 & 0 & 0 & 1 & 1 & 1 & 1 & 1 & 0 & 0 & 1 & 1 & 1 & 1 & 0 & 0 & 1 & 1 \\
\hline $\mathbf{8 0}$ & 0 & 0 & 0 & 0 & 0 & 0 & 1 & 0 & 0 & 0 & 0 & 0 & 1 & 1 & 1 & 1 & 0 & 0 & 1 \\
\hline $\mathbf{9 0}$ & 0 & 0 & 0 & 0 & 0 & 0 & 0 & 1 & 0 & 0 & 0 & 0 & 0 & 1 & 0 & 0 & 0 & 0 & 0 \\
\hline $\mathbf{1 0 0}$ & 0 & 0 & 0 & 0 & 0 & 0 & 0 & 1 & 1 & 0 & 0 & 1 & 1 & 1 & 1 & 0 & 0 & 0 & 1 \\
\hline $\mathbf{1 1 0}$ & 0 & 0 & 0 & 0 & 0 & 0 & 0 & 1 & 1 & 1 & 1 & 1 & 0 & 1 & 1 & 0 & 0 & 0 & 1 \\
\hline $\mathbf{1 2 0}$ & 0 & 0 & 0 & 0 & 0 & 0 & 0 & 0 & 0 & 0 & 1 & 1 & 0 & 0 & 0 & 0 & 0 & 0 & 0 \\
\hline $\mathbf{1 3 0}$ & 0 & 0 & 0 & 0 & 0 & 0 & 0 & 0 & 0 & 0 & 0 & 1 & 0 & 0 & 0 & 1 & 0 & 0 & 0 \\
\hline $\mathbf{1 4 0}$ & 0 & 0 & 0 & 0 & 0 & 0 & 0 & 0 & 0 & 0 & 0 & 1 & 1 & 0 & 1 & 1 & 0 & 0 & 1 \\
\hline $\mathbf{1 5 0}$ & 0 & 0 & 0 & 0 & 0 & 0 & 0 & 0 & 0 & 0 & 0 & 1 & 1 & 1 & 1 & 0 & 0 & 0 & 1 \\
\hline $\mathbf{1 6 0}$ & 0 & 0 & 0 & 0 & 0 & 0 & 0 & 0 & 0 & 0 & 0 & 1 & 0 & 0 & 1 & 0 & 0 & 0 & 1 \\
\hline $\mathbf{1 7 0}$ & 0 & 0 & 0 & 0 & 0 & 0 & 0 & 0 & 0 & 0 & 0 & 0 & 0 & 0 & 0 & 1 & 0 & 0 & 0 \\
\hline $\mathbf{1 0}$ & 1 & 1 & 1 & 1 & 1 & 1 & 1 & 1 & 1 & 1 & 1 & 1 & 1 & 1 & 1 & 0 & 1 & 1 & 1 \\
\hline $\mathbf{6 5}$ & 0 & 0 & 0 & 0 & 1 & 0 & 1 & 1 & 0 & 0 & 0 & 0 & 1 & 0 & 1 & 0 & 0 & 1 & 1 \\
\hline $\mathbf{1 6 5}$ & 0 & 0 & 0 & 0 & 0 & 0 & 0 & 0 & 0 & 0 & 0 & 1 & 0 & 0 & 1 & 0 & 0 & 0 & 1 \\
\hline
\end{tabular}

Matriz Poluição para Extensão da Contaminação (nível de serviço: 15.00\%):

\begin{tabular}{|c|c|c|c|c|c|c|c|c|c|c|c|c|c|c|c|c|c|c|c|c|c|}
\hline Nós & $\mathbf{2 0}$ & $\mathbf{3 0}$ & $\mathbf{4 0}$ & $\mathbf{5 0}$ & $\mathbf{6 0}$ & $\mathbf{7 0}$ & $\mathbf{8 0}$ & $\mathbf{9 0}$ & $\mathbf{1 0 0}$ & $\mathbf{1 1 0}$ & $\mathbf{1 2 0}$ & $\mathbf{1 3 0}$ & $\mathbf{1 4 0}$ & $\mathbf{1 5 0}$ & $\mathbf{1 6 0}$ & $\mathbf{1 7 0}$ & $\mathbf{1 0}$ & $\mathbf{6 5}$ & $\mathbf{1 6 5}$ \\
\hline $\mathbf{2 0}$ & 1 & 1 & 1 & 1 & 1 & 1 & 1 & 1 & 1 & 1 & 1 & 1 & 1 & 1 & 1 & 1 & 0 & 1 & 1 \\
\hline $\mathbf{3 0}$ & 0 & 1 & 1 & 1 & 1 & 0 & 1 & 1 & 1 & 0 & 0 & 1 & 1 & 1 & 1 & 1 & 0 & 1 & 1 \\
\hline $\mathbf{4 0}$ & 0 & 0 & 1 & 1 & 0 & 0 & 1 & 1 & 1 & 0 & 0 & 1 & 1 & 1 & 1 & 1 & 0 & 0 & 1 \\
\hline $\mathbf{5 0}$ & 0 & 0 & 0 & 1 & 0 & 0 & 1 & 1 & 1 & 0 & 0 & 1 & 1 & 1 & 1 & 1 & 0 & 0 & 1 \\
\hline $\mathbf{6 0}$ & 0 & 0 & 0 & 0 & 1 & 0 & 1 & 1 & 1 & 0 & 0 & 1 & 1 & 1 & 1 & 1 & 0 & 1 & 1 \\
\hline $\mathbf{7 0}$ & 0 & 1 & 1 & 1 & 1 & 1 & 1 & 1 & 1 & 0 & 0 & 1 & 1 & 1 & 1 & 1 & 0 & 1 & 1 \\
\hline $\mathbf{8 0}$ & 0 & 0 & 0 & 0 & 0 & 0 & 1 & 1 & 1 & 0 & 0 & 1 & 1 & 1 & 1 & 1 & 0 & 0 & 1 \\
\hline $\mathbf{9 0}$ & 0 & 0 & 0 & 0 & 0 & 0 & 1 & 1 & 1 & 0 & 0 & 1 & 1 & 1 & 1 & 1 & 0 & 0 & 1 \\
\hline $\mathbf{1 0 0}$ & 0 & 0 & 0 & 0 & 0 & 0 & 1 & 1 & 1 & 0 & 0 & 1 & 1 & 1 & 1 & 1 & 0 & 0 & 1 \\
\hline $\mathbf{1 1 0}$ & 0 & 0 & 0 & 0 & 0 & 0 & 1 & 1 & 1 & 1 & 1 & 1 & 1 & 1 & 1 & 1 & 0 & 0 & 1 \\
\hline $\mathbf{1 2 0}$ & 0 & 0 & 0 & 0 & 0 & 0 & 1 & 1 & 1 & 0 & 1 & 1 & 1 & 1 & 1 & 1 & 0 & 0 & 1 \\
\hline $\mathbf{1 3 0}$ & 0 & 0 & 0 & 0 & 0 & 0 & 1 & 1 & 1 & 0 & 0 & 1 & 1 & 1 & 1 & 1 & 0 & 0 & 1 \\
\hline $\mathbf{1 4 0}$ & 0 & 0 & 0 & 0 & 0 & 0 & 1 & 1 & 1 & 0 & 0 & 1 & 1 & 1 & 1 & 1 & 0 & 0 & 1 \\
\hline $\mathbf{1 5 0}$ & 0 & 0 & 0 & 0 & 0 & 0 & 1 & 1 & 1 & 0 & 0 & 1 & 1 & 1 & 1 & 1 & 0 & 0 & 1 \\
\hline $\mathbf{1 6 0}$ & 0 & 0 & 0 & 0 & 0 & 0 & 1 & 1 & 1 & 0 & 0 & 1 & 1 & 1 & 1 & 1 & 0 & 0 & 1 \\
\hline $\mathbf{1 7 0}$ & 0 & 0 & 0 & 0 & 0 & 0 & 1 & 1 & 1 & 0 & 0 & 1 & 1 & 1 & 1 & 1 & 0 & 0 & 1 \\
\hline $\mathbf{1 0}$ & 1 & 1 & 1 & 1 & 1 & 1 & 1 & 1 & 1 & 1 & 1 & 1 & 1 & 1 & 1 & 1 & 1 & 1 & 1 \\
\hline $\mathbf{6 5}$ & 0 & 0 & 0 & 0 & 1 & 0 & 1 & 1 & 1 & 0 & 0 & 1 & 1 & 1 & 1 & 1 & 0 & 1 & 1 \\
\hline $\mathbf{1 6 5}$ & 0 & 0 & 0 & 0 & 0 & 0 & 1 & 1 & 1 & 0 & 0 & 1 & 1 & 1 & 1 & 1 & 0 & 0 & 1 \\
\hline
\end{tabular}

Programa finalizado com sucesso!

Tempo decorrido: 10.65 s. 


\section{Anexo VI - PLEDECORDA_MOM.TXT (Rede Net1):}

\section{Saídas dos Nós escolhidos:}

\section{Nível de Serviço 1 - Volume Consumido: 468,10m³}

\section{FRENTES PARETO GLOBAL}

Número de Equipamentos: 1 un.

Considerado acréscimo de 0.01 por detecções repetidas.

$\begin{array}{lllll} & \text { f1: } & \text { f2: } & \text { f3: } & \text { Nós de monitoramento (X[i]): } \\ \text { 1: } & 7 & 9 & 19 & 90(7) \\ \text { 2: } & 6 & 13 & 19 & 160(14)\end{array}$

Número de Equipamentos: 2 un.

Considerado acréscimo de 0.01 por detecções repetidas.

\begin{tabular}{llllll} 
& f1: & f2: & f3: & \multicolumn{2}{c}{ Nós de monitoramento (X[i]): } \\
1: & 13 & 15.07 & 19.19 & $90(7)$ & $160(14)$ \\
2: & 8 & 17.06 & 19.19 & $130(11)$ & $140(12)$ \\
3: & 10 & 17.03 & 19.19 & $80(6)$ & $130(11)$
\end{tabular}

Número de Equipamentos: 3 un.

Considerado acréscimo de 0.01 por detecções repetidas.

\begin{tabular}{lllllll} 
& f1: & f2: & f3: & \multicolumn{2}{c}{ Nós de monitoramento (X[i]): } \\
1: & 15 & 18.14 & 19.38 & $130(11)$ & $90(7)$ & $140(12)$ \\
2: & 16.03 & 16.15 & 19.38 & $160(14)$ & $80(6)$ & $90(7)$ \\
3: & 16.01 & 17.09 & 19.38 & $160(14)$ & $90(7)$ & $170(15)$ \\
4: & 15.02 & 17.16 & 19.38 & $160(14)$ & $90(7)$ & $130(11)$
\end{tabular}

Número de Equipamentos: 4 un.

Considerado acréscimo de 0.01 por detecções repetidas.
f1: f2: f3:
Nós de monitoramento (X[i]):
$\begin{array}{llll}1: & 16.05 & 19.14 & 19.57\end{array}$
$130(11)$
$80(6)$
$90(7)$ 


$\begin{array}{lrllllll}\text { 2: } & 17.04 & 18.27 & 19.57 & 130(11) & 90(7) & 140(12) & 160(14) \\ \text { 3: } & 18.05 & 18.24 & 19.57 & 130(11) & 80(6) & 90(7) & 160(14) \\ \text { 4: } & 16.03 & 19.17 & 19.57 & 130(11) & 90(7) & 140(12) & 170(15) \\ \text { 5: } & 19.04 & 18.17 & 19.57 & 160(14) & 80(6) & 90(7) & 170(15)\end{array}$

\section{FRENTES PARETO GLOBAL}

Número de Equipamentos: 1 un.

Não considerado acréscimo por detecções repetidas.

$\begin{array}{lllll} & \text { f1: } & \text { f2: } & \text { f3: } & \text { Nós de monitoramento (X[i]): } \\ 1: & 6 & 13 & 19 & 160(14) \\ 2: & 7 & 9 & 19 & 90(7)\end{array}$

Número de Equipamentos: 2 un.

Não considerado acréscimo por detecções repetidas.

\begin{tabular}{lccccc} 
& f1: & f2: & f3: & \multicolumn{2}{c}{ Nós de monitoramento (X[i]): } \\
1: & 13 & 15 & 19 & $160(14)$ & $90(7)$ \\
2: & 10 & 17 & 19 & $130(11)$ & $80(6)$
\end{tabular}

Número de Equipamentos: 3 un.

Não considerado acréscimo por detecções repetidas.

f1: f2: f3: $\quad$ Nós de monitoramento (X[i]):
1: $\quad 15 \quad 18 \quad 19$
$140(12)$
$90(7)$
$130(11)$
2: $\quad 16 \quad 17 \quad 19$
$90(7)$
$170(15)$
$160(14)$

Número de Equipamentos: 4 un.

Não considerado acréscimo por detecções repetidas.
f1: f2: f3:
Nós de monitoramento (X[i]):
1: $\quad 16 \quad 19 \quad 19$
$130(11)$
$170(15)$
$90(7)$
$80(6)$
2: $\quad 19 \quad 18 \quad 19$
$90(7) \quad 160(14)$
$170(15)$
$80(6)$ 


\section{Anexo VII - PLEDECORDA_EPA.TXT (Rede Anytown): Saídas das Matrizes de Poluição: \\ Nível de Serviço 1 - Volume Consumido: 1245,15m³}

PLEDECORDA - Programa de Localização de Equipamentos para DEtecção de COntaminação em Rede de Distribuição de Água

Programador: Luiz Fernando de Souza Dias.

Orientadora: Luisa Fernanda Ribeiro Reis.

Data Inicial do Programa: 15/03/2005

Data e hora do início da execução: 02/16/06 14:11:36

Número de tubos da rede: 34

Número de nós da rede: 19

Número de reservatórios e tanques da rede: 3

Duração do período da Simulação (h): 240

Comprimento total da rede (ft): 228200

TUBO (1): 2 Nó inicial (1): 20 Nó final (6) : 70 Compr.(ft): 12000.00 Diam.(in): 16.00

* Soma+: 4121963.25

* Soma-: $\quad 0.00$

* Contador: 241

TUBO (2): 4 Nó inicial (1): 20 Nó final (2) : 30 Compr.(ft): 12000.00 Diam.(in): 12.00

* Soma+: 3453652.50

* Soma-: 0.00

* Contador: 241

TUBO (3): 6 Nó inicial (1): 20 Nó final (10) : 110 Compr.(ft): 12000.00 Diam.(in): 12.00

* Soma+: 3700607.75

* Soma-: 0.00

* Contador: 241

TUBO (4): 8 Nó inicial (2): 30 Nó final (6) : 70 Compr.(ft): 9000.00 Diam.(in): 12.00

* Soma+: $\quad 0.00$

* Soma-: -298116.16

* Contador: 241

TUBO (5): 10 Nó inicial (6): 70 Nó final (9) : 100 Compr.(ft): 6000.00 Diam.(in): 12.00

* Soma+: 2309610.25

* Soma-: 0.00

* Contador: 241

TUBO (6): 12 Nó inicial (6): 70 Nó final (8) : 90 Compr.(ft): 6000.00 Diam.(in): 10.00

* Soma+: 2158205.75 
Estudo da Localização Otimizada de Equipamentos em Rede de Distribuição de Água para Deteç̧ão de Contaminação

* Soma-: $\quad 0.00$

* Contador: 241

TUBO (7): 14 Nó inicial (6): 70 Nó final (5) : 60 Compr.(ft): 6000.00 Diam.(in): 12.00

* Soma+: 1992290.62

* Soma-: 0.00

* Contador: 241

TUBO (8): 16 Nó inicial (5): 60 Nó final (8) : 90 Compr.(ft): 6000.00 Diam.(in): 10.00

* Soma+: 1047277.31

* Soma-: 0.00

* Contador: 241

TUBO (9): 18 Nó inicial (5): 60 Nó final (7) : 80 Compr.(ft): 6000.00 Diam.(in): 12.00

* Soma+: 1189309.25

* Soma-: $\quad 0.00$

* Contador: 241

TUBO (10): 20 Nó inicial (7): 80 Nó final (8) : 90 Compr.(ft): 6000.00 Diam.(in): 10.00

* Soma+: 99212.45

* Soma-: -69697.99

* Contador: 241

TUBO (11): 22 Nó inicial (14): 150 Nó final (8) : 90 Compr.(ft): 6000.00 Diam.(in): 10.00

* Soma+: 27913.84

* Soma-: -254836.89

* Contador: 241

TUBO (12): 24 Nó inicial (9): 100 Nó final (8) : 90 Compr.(ft): 6000.00 Diam.(in): 10.00

* Soma+: 546463.06

* Soma-: -14553.72

* Contador: 241

TUBO (13): 26 Nó inicial (14): 150 Nó final (9) : 100 Compr.(ft): 6000.00 Diam.(in): 12.00

* Soma+: $\quad 0.00$

* Soma-: -725017.00

* Contador: 241

TUBO (14): 28 Nó inicial (7): 80 Nó final (14) : 150 Compr.(ft): 6000.00 Diam.(in): 10.00

* Soma+: 279434.81

* Soma-: $\quad-8471.83$

* Contador: 241

TUBO (15): 30 Nó inicial (2): 30 Nó final (5) : 60 Compr.(ft): 6000.00 Diam.(in): 10.00

* Soma+: 1731744.62

* Soma-: $\quad 0.00$

* Contador: 241 
Estudo da Localização Otimizada de Equipamentos em Rede de Distribuição de Água para Detecção de Contaminação

TUBO (16): 32 Nó inicial (2): 30 Nó final (3) : 40 Compr.(ft): 6000.00 Diam.(in): 10.00

* Soma+: 1532301.75

* Soma-: 0.00

* Contador: 241

TUBO (17): 34 Nó inicial (2): 30 Nó final (4) : 50 Compr.(ft): 9000.00 Diam.(in): 10.00

* Soma+: 1430503.12

* Soma-: 0.00

* Contador: 241

TUBO (18): 36 Nó inicial (3): 40 Nó final (4) : 50 Compr.(ft): 6000.00 Diam.(in): 10.00

* Soma+: 824305.81

* Soma-: 0.00

* Contador: 241

TUBO (19): 38 Nó inicial (4): 50 Nó final (7) : 80 Compr.(ft): 6000.00 Diam.(in): 10.00

* Soma+: 1050752.50

* Soma-: 0.00

* Contador: 241

TUBO (20): 40 Nó inicial (13): 140 Nó final (7) : 80 Compr.(ft): 6000.00 Diam.(in): 10.00

* Soma+: $\quad 0.00$

* Soma-: -692889.38

* Contador: 241

TUBO (21): 42 Nó inicial (13): 140 Nó final (14) : 150 Compr.(ft): 6000.00 Diam.(in): 8.00

* Soma+: $\quad 0.00$

* Soma-: -536864.81

* Contador: 241

TUBO (22): 44 Nó inicial (14): 150 Nó final (15) : 160 Compr.(ft): 6000.00 Diam.(in): 8.00

* Soma+: 826505.00

* Soma-: -60379.48

* Contador: 241

TUBO (23): 46 Nó inicial (15): 160 Nó final (9) : 100 Compr.(ft): 6000.00 Diam.(in): 8.00

* Soma+: 18600.40

* Soma-: -1042251.75

* Contador: 241

TUBO (24): 48 Nó inicial (10): 110 Nó final (9) : 100 Compr.(ft): 6000.00 Diam.(in): 8.00

* Soma+: 1055033.00

* Soma-: $\quad 0.00$

* Contador: 241

TUBO (25): 50 Nó inicial (10): 110 Nó final (15) : 160 Compr.(ft): 6000.00 Diam.(in): 10.00

* Soma+: 1833835.12 
Estudo da Localização Otimizada de Equipamentos em Rede de Distribuição de Água para Deteç̧ão de Contaminação

* Soma-: $\quad 0.00$

* Contador: 241

TUBO (26): 52 Nó inicial (11): 120 Nó final (10) : 110 Compr.(ft): 6000.00 Diam.(in): 8.00

* Soma+: $\quad 0.00$

* Soma-: -1640359.12

* Contador: 241

TUBO (27): 56 Nó inicial (12): 130 Nó final (11) : 120 Compr.(ft): 6000.00 Diam.(in): 8.00

* Soma+: $\quad 0.00$

* Soma-: -534114.38

* Contador: 241

TUBO (28): 58 Nó inicial (12): 130 Nó final (15) : 160 Compr.(ft): 6000.00 Diam.(in): 10.00

* Soma+: 63318.39

* Soma-: -646605.50

* Contador: 241

TUBO (29): 60 Nó inicial (16): 170 Nó final (12) : 130 Compr.(ft): 6000.00 Diam.(in): 8.00

* Soma+: 43074.71

* Soma-: -382330.75

* Contador: 241

TUBO (30): 62 Nó inicial (13): 140 Nó final (15) : 160 Compr.(ft): 6000.00 Diam.(in): 8.00

* Soma+: 639524.62

* Soma-: -118161.22

* Contador: 241

TUBO (31): 64 Nó inicial (13): 140 Nó final (16) : 170 Compr.(ft): 12000.00 Diam.(in): 8.00

* Soma+: 766988.38

* Soma-: 0.00

* Contador: 241

TUBO (32): 66 Nó inicial (4): 50 Nó final (13) : 140 Compr.(ft): 12000.00 Diam.(in): 8.00

* Soma+: 775092.19

* Soma-: 0.00

* Contador: 241

TUBO (33): 78 Nó inicial (18): 65 Nó final (5) : 60 Compr.(ft): 100.00 Diam.(in): 12.00

* Soma+: 525809.12

* Soma-: -574953.56

* Contador: 217

TUBO (34): 80 Nó inicial (15): 160 Nó final (19) : 165 Compr.(ft): 100.00 Diam.(in): 12.00

* Soma+: 697376.44

* Soma-: -768424.44

* Contador: 185 
Estudo da Localização Otimizada de Equipamentos em Rede de Distribuição de Água para Deteç̧ão de Contaminação

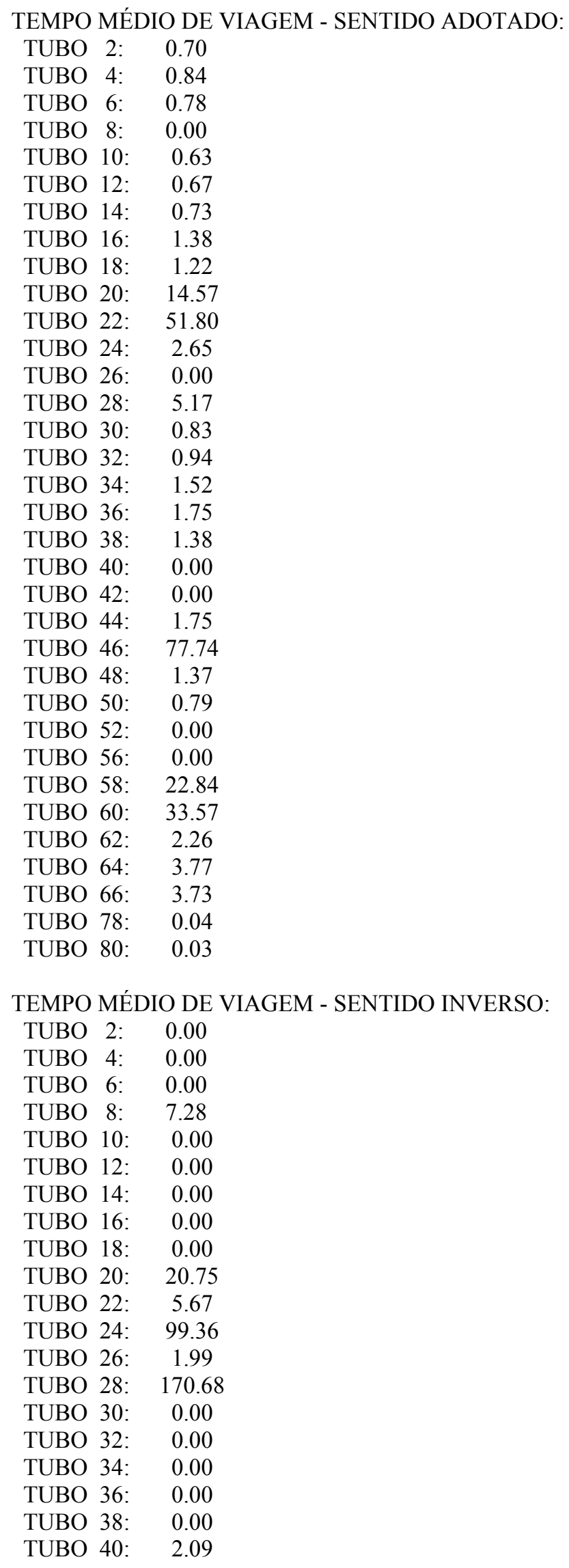


Estudo da Localização Otimizada de Equipamentos em Rede de Distribuição de Água para Deteç̧ão de Contaminação

TUBO 42: $\quad 2.69$

TUBO 44: 23.95

TUBO 46: 1.39

TUBO 48: $\quad 0.00$

TUBO 50: $\quad 0.00$

TUBO 52: $\quad 0.88$

TUBO 56: 2.71

TUBO 58: $\quad 2.24$

TUBO 60: 3.78

TUBO 62: $\quad 12.24$

TUBO 64: $\quad 0.00$

TUBO 66: $\quad 0.00$

TUBO 78: $\quad 0.04$

TUBO 80: 0.02

Matriz Custo de Tempo de viagem Inicial (h):

\begin{tabular}{|c|c|c|c|c|c|c|c|c|c|c|c|c|c|c|c|c|c|c|c|}
\hline Nós & 20 & 30 & 40 & 50 & 60 & 70 & 80 & 90 & 100 & 110 & 120 & 130 & 140 & 150 & 160 & 170 & 10 & 65 & 165 \\
\hline 20 & 0 & 0.84 & 888 & 888 & 888 & 0.70 & 8888 & 888 & 888 & 0.78 & 888 & 888 & 888 & 888 & $\begin{array}{lll}8 & 88\end{array}$ & 888 & 888 & 888 & 888 \\
\hline 30 & 888 & 0 & 0.94 & 1.52 & 0.83 & 888 & 888 & 888 & 888 & 888 & 888 & 888 & 888 & 888 & 888 & 888 & 888 & 888 & 888 \\
\hline 40 & 888 & 888 & 0 & 1.75 & 888 & 888 & 888 & 888 & 888 & 888 & 888 & 888 & 888 & 888 & $\begin{array}{lll}8 & 8 & 8\end{array}$ & 888 & 888 & 888 & 888 \\
\hline 50 & 888 & 888 & 888 & 0 & 888 & 888 & 1.38 & 888 & 888 & 888 & 888 & 888 & 3.73 & 888 & 888 & 888 & 888 & 888 & 888 \\
\hline 60 & 888 & 888 & 888 & 888 & 0 & 888 & 1.22 & 1.38 & 888 & 888 & 888 & 888 & 888 & 888 & 8888 & 888 & 888 & 0.04 & 888 \\
\hline 70 & 888 & 7.28 & 888 & 888 & 0.73 & 0 & 888 & 0.67 & 0.63 & 888 & 888 & 8888 & 888 & 888 & 888 & 888 & 888 & 888 & 888 \\
\hline 80 & 888 & 888 & 888 & 888 & 888 & 888 & 0 & 14.57 & 888 & 888 & 888 & 888 & 2.09 & 5.17 & 888 & 888 & 888 & 888 & 888 \\
\hline 90 & 888 & 888 & 888 & 888 & 888 & 888 & 20.75 & 0 & 99.36 & 888 & 888 & 888 & 888 & 5.67 & 8888 & 888 & 888 & 888 & 888 \\
\hline 100 & 888 & 888 & 888 & 888 & 888 & 888 & 888 & 2.65 & 0 & 888 & 888 & 8888 & 8888 & 1.99 & 1.39 & 888 & 888 & 888 & 888 \\
\hline 110 & 888 & 888 & 888 & 888 & 888 & 888 & 888 & 888 & 1.37 & 0 & 0.88 & 888 & 888 & 888 & 0.79 & 888 & 888 & 888 & 888 \\
\hline 120 & 888 & 888 & 888 & 888 & 888 & 888 & 888 & 888 & 888 & 888 & 0 & 2.71 & 888 & 888 & $\begin{array}{llll}8 & 8 & 8\end{array}$ & 888 & 888 & 888 & 888 \\
\hline 130 & 888 & 888 & 888 & 888 & 888 & 888 & 888 & 888 & 888 & 888 & 888 & 0 & 888 & 888 & 22.84 & 3.78 & 888 & 888 & 888 \\
\hline 140 & 888 & 888 & 888 & 888 & 888 & 888 & 888 & 888 & 888 & 888 & 888 & $\begin{array}{lll}8 & 88 \\
\end{array}$ & 0 & 888 & 2.26 & 3.77 & 888 & 888 & 888 \\
\hline 150 & 888 & 888 & 888 & 888 & 888 & 888 & 170.68 & 51.80 & 888 & 888 & 888 & 888 & 2.69 & 0 & 1.75 & 888 & 888 & 888 & 888 \\
\hline 160 & 888 & 888 & 888 & 888 & 888 & 888 & 888 & 8888 & 77.74 & 888 & 888 & 2.24 & 12.24 & 23.95 & 0 & 888 & 888 & 888 & 0.03 \\
\hline 170 & 888 & 888 & 888 & 888 & 888 & 888 & 888 & 888 & 8888 & 888 & 888 & 33.57 & 8888 & 888 & 888 & 0 & 888 & 888 & 888 \\
\hline 10 & 0 & 888 & 888 & 888 & 888 & 888 & 888 & 888 & 888 & 888 & 888 & 888 & 888 & 888 & 888 & 888 & 0 & 888 & 888 \\
\hline 65 & 888 & 888 & 888 & 888 & 0.04 & 888 & 888 & 888 & 888 & 888 & 888 & $\begin{array}{llll}8 & 8 & 8\end{array}$ & $\begin{array}{llll}8 & 8 & 8\end{array}$ & 888 & 8888 & 888 & 888 & 0 & 888 \\
\hline 165 & 888 & 888 & 888 & 888 & 888 & 888 & 888 & 888 & 888 & 888 & 888 & 8888 & 8888 & 888 & 0.02 & 888 & 888 & 888 & 0 \\
\hline
\end{tabular}


Estudo da Localização Otimizada de Equipamentos em Rede de Distribuição de Água para Deteç̧ão de Contaminação

Matriz Roteamento Inicial 1:

\begin{tabular}{|c|c|c|c|c|c|c|c|c|c|c|c|c|c|c|c|c|c|c|c|}
\hline Nós & $\mathbf{2 0}$ & $\mathbf{3 0}$ & $\mathbf{4 0}$ & $\mathbf{5 0}$ & $\mathbf{6 0}$ & $\mathbf{7 0}$ & $\mathbf{8 0}$ & $\mathbf{9 0}$ & $\mathbf{1 0 0}$ & $\mathbf{1 1 0}$ & $\mathbf{1 2 0}$ & $\mathbf{1 3 0}$ & $\mathbf{1 4 0}$ & $\mathbf{1 5 0}$ & $\mathbf{1 6 0}$ & $\mathbf{1 7 0}$ & $\mathbf{1 0}$ & $\mathbf{6 5}$ & $\mathbf{1 6 5}$ \\
\hline $\mathbf{2 0}$ & 1 & 2 & 0 & 0 & 0 & 6 & 0 & 0 & 0 & $\mathbf{1 0}$ & 0 & 0 & 0 & 0 & 0 & 0 & 0 & 0 & 0 \\
\hline $\mathbf{3 0}$ & 0 & 2 & 3 & 4 & 5 & 0 & 0 & 0 & 0 & 0 & 0 & 0 & 0 & 0 & 0 & 0 & 0 & 0 & 0 \\
\hline $\mathbf{4 0}$ & 0 & 0 & 3 & 4 & 0 & 0 & 0 & 0 & 0 & 0 & 0 & 0 & 0 & 0 & 0 & 0 & 0 & 0 & 0 \\
\hline $\mathbf{5 0}$ & 0 & 0 & 0 & 4 & 0 & 0 & 7 & 0 & 0 & 0 & 0 & 0 & 13 & 0 & 0 & 0 & 0 & 0 & 0 \\
\hline $\mathbf{6 0}$ & 0 & 0 & 0 & 0 & 5 & 0 & 7 & 8 & 0 & 0 & 0 & 0 & 0 & 0 & 0 & 0 & 0 & 18 & 0 \\
\hline $\mathbf{7 0}$ & 0 & 2 & 0 & 0 & 5 & 6 & 0 & 8 & 9 & 0 & 0 & 0 & 0 & 0 & 0 & 0 & 0 & 0 & 0 \\
\hline $\mathbf{8 0}$ & 0 & 0 & 0 & 0 & 0 & 0 & 7 & 8 & 0 & 0 & 0 & 0 & 13 & 14 & 0 & 0 & 0 & 0 & 0 \\
\hline $\mathbf{9 0}$ & 0 & 0 & 0 & 0 & 0 & 0 & 7 & 8 & 9 & 0 & 0 & 0 & 0 & 14 & 0 & 0 & 0 & 0 & 0 \\
\hline $\mathbf{1 0 0}$ & 0 & 0 & 0 & 0 & 0 & 0 & 0 & 8 & 9 & 0 & 0 & 0 & 0 & 14 & 15 & 0 & 0 & 0 & 0 \\
\hline $\mathbf{1 1 0}$ & 0 & 0 & 0 & 0 & 0 & 0 & 0 & 0 & 9 & 10 & 11 & 0 & 0 & 0 & 15 & 0 & 0 & 0 & 0 \\
\hline $\mathbf{1 2 0}$ & 0 & 0 & 0 & 0 & 0 & 0 & 0 & 0 & 0 & 0 & 11 & 12 & 0 & 0 & 0 & 0 & 0 & 0 & 0 \\
\hline $\mathbf{1 3 0}$ & 0 & 0 & 0 & 0 & 0 & 0 & 0 & 0 & 0 & 0 & 0 & 12 & 0 & 0 & 15 & 16 & 0 & 0 & 0 \\
\hline $\mathbf{1 4 0}$ & 0 & 0 & 0 & 0 & 0 & 0 & 0 & 0 & 0 & 0 & 0 & 0 & 13 & 0 & 15 & 16 & 0 & 0 & 0 \\
\hline $\mathbf{1 5 0}$ & 0 & 0 & 0 & 0 & 0 & 0 & 7 & 8 & 0 & 0 & 0 & 0 & 13 & 14 & 15 & 0 & 0 & 0 & 0 \\
\hline $\mathbf{1 6 0}$ & 0 & 0 & 0 & 0 & 0 & 0 & 0 & 0 & 9 & 0 & 0 & 12 & 13 & 14 & 15 & 0 & 0 & 0 & 19 \\
\hline $\mathbf{1 7 0}$ & 0 & 0 & 0 & 0 & 0 & 0 & 0 & 0 & 0 & 0 & 0 & 12 & 0 & 0 & 0 & 16 & 0 & 0 & 0 \\
\hline $\mathbf{1 0}$ & 1 & 0 & 0 & 0 & 0 & 0 & 0 & 0 & 0 & 0 & 0 & 0 & 0 & 0 & 0 & 0 & 17 & 0 & 0 \\
\hline $\mathbf{6 5}$ & 0 & 0 & 0 & 0 & 5 & 0 & 0 & 0 & 0 & 0 & 0 & 0 & 0 & 0 & 0 & 0 & 0 & 18 & 0 \\
\hline $\mathbf{1 6 5}$ & 0 & 0 & 0 & 0 & 0 & 0 & 0 & 0 & 0 & 0 & 0 & 0 & 0 & 0 & 15 & 0 & 0 & 0 & 19 \\
\hline
\end{tabular}

Matriz Custo de Tempo de viagem Final (h) (Caminhos Mais Curtos):

\begin{tabular}{|c|c|c|c|c|c|c|c|c|c|c|c|c|c|c|c|c|c|c|c|}
\hline Nós & 20 & 30 & 40 & 50 & 60 & 70 & 80 & 90 & 100 & 110 & 120 & 130 & 140 & 150 & 160 & 170 & 10 & 65 & 165 \\
\hline 20 & 0 & .84 & .78 & .35 & 1.43 & 0.70 & 2.64 & .37 & 1.33 & 0.78 & 1.66 & 3.81 & 4.73 & 3.32 & 1.57 & 7.59 & 888 & 1.47 & 1.60 \\
\hline 30 & 388 & 0 & 0.94 & 1.52 & 0.83 & 388 & 2.05 & .22 & 84.14 & 888 & 888 & 8.64 & 4.14 & 7.23 & 6.40 & 7.91 & 888 & 0.87 & 6.43 \\
\hline 40 & 888 & 888 & 0 & 1.75 & 888 & 888 & 3.13 & 17.71 & 85.22 & 888 & 888 & 9.71 & 5.22 & 8.31 & 7.48 & 8.99 & 888 & 888 & 7.50 \\
\hline 50 & 888 & 888 & 888 & 0 & 888 & 888 & 1.38 & 15.95 & 83.46 & 888 & 888 & 7.96 & 3.46 & 6.55 & 5.72 & 7.23 & 888 & 888 & 5.75 \\
\hline 60 & 888 & 888 & 888 & 888 & 0 & 888 & 1.22 & 1.38 & 83.30 & 888 & 888 & 7.80 & 3.30 & 6.39 & 5.56 & 7.07 & 888 & 0.04 & 5.59 \\
\hline 70 & 888 & 7.28 & 8.22 & 8.79 & 0.73 & 0 & 1.94 & 0.67 & 0.63 & 888 & 888 & 4.25 & 4.03 & 2.62 & 2.01 & 7.80 & 888 & 0.76 & 2.04 \\
\hline 80 & 888 & 888 & 888 & 888 & 888 & 888 & 0 & 14.57 & 82.09 & 888 & 888 & 6.58 & 2.09 & 5.17 & 4.35 & 5.86 & 888 & 888 & 4.37 \\
\hline 90 & 888 & 888 & 888 & 888 & 888 & 888 & 20.75 & 0 & 85.16 & 888 & 888 & 9.66 & 8.37 & 5.67 & 7.42 & 12.14 & 888 & 888 & 7.45 \\
\hline 100 & 888 & 888 & 888 & 888 & 888 & 888 & 23.39 & 2.65 & 0 & 888 & 888 & 3.62 & 4.69 & 1.99 & 1.39 & 7.41 & 888 & 888 & 1.41 \\
\hline 110 & 888 & 888 & 888 & 888 & 888 & 888 & 24.76 & 4.02 & 1.37 & 0 & 0.88 & 3.02 & 6.06 & 3.37 & 0.79 & 6.81 & 888 & 888 & 0.82 \\
\hline 120 & 888 & 888 & 888 & 888 & 888 & 888 & 122.04 & 101.30 & 103.28 & 000 & 0 & 2.71 & 37.78 & 49.49 & 25.54 & 6.49 & 888 & 888 & 25.57 \\
\hline 130 & 888 & 888 & 888 & 888 & 888 & 888 & 119.33 & 98.59 & 100.58 & & 888 & 0 & 35.07 & 46.79 & 22.84 & 3.78 & 888 & 888 & 22.86 \\
\hline 140 & 888 & 888 & 888 & 888 & 888 & 888 & 98.76 & 78.01 & 80 & 888 & 888 & 4.50 & 0 & 26.21 & 2.26 & 3.77 & 888 & 888 & 2.29 \\
\hline 150 & 888 & 888 & 888 & 888 & 888 & 888 & 72.55 & 51.80 & 79.49 & 888 & 888 & 3.99 & 2.69 & 0 & 1.75 & 6.46 & 888 & 888 & 1.78 \\
\hline 160 & 888 & 888 & 888 & 888 & 888 & 888 & 96.50 & 75.75 & 77.74 & 888 & 888 & 2.24 & 12.24 & 23.95 & 0 & 6.02 & 888 & 888 & 0.03 \\
\hline 170 & 888 & 888 & 888 & 888 & 888 & 888 & 152.90 & 132.16 & 134.15 & 888 & 888 & 33.57 & 68.64 & 80.36 & 56.41 & 0 & 888 & 888 & 56.43 \\
\hline 10 & 0 & 0.84 & 1.78 & 2.35 & 1.43 & 0.70 & 2.64 & 1.37 & 1.33 & 0.78 & 1.66 & 3.81 & 4.73 & 3.32 & 1.57 & 7.59 & 0 & 1.47 & 1.60 \\
\hline 65 & 888 & 888 & 888 & 888 & 0.04 & 888 & 1.26 & 1.42 & 83.35 & 888 & 888 & 7.84 & 3.34 & 6.43 & 5.61 & 7.11 & 888 & 0 & 5.63 \\
\hline 165 & 888 & 888 & 888 & 888 & 888 & 888 & 96.52 & 75.77 & 77.76 & 888 & 888 & 2.26 & 12.26 & 23.97 & 0.02 & 6.04 & 888 & 888 & 0 \\
\hline
\end{tabular}


Estudo da Localização Otimizada de Equipamentos em Rede de Distribuição de Água para Deteç̧ão de Contaminação

Matriz Roteamento Final:

\begin{tabular}{|c|c|c|c|c|c|c|c|c|c|c|c|c|c|c|c|c|c|c|c|}
\hline Nós & $\mathbf{2 0}$ & $\mathbf{3 0}$ & $\mathbf{4 0}$ & $\mathbf{5 0}$ & $\mathbf{6 0}$ & $\mathbf{7 0}$ & $\mathbf{8 0}$ & $\mathbf{9 0}$ & $\mathbf{1 0 0}$ & $\mathbf{1 1 0}$ & $\mathbf{1 2 0}$ & $\mathbf{1 3 0}$ & $\mathbf{1 4 0}$ & $\mathbf{1 5 0}$ & $\mathbf{1 6 0}$ & $\mathbf{1 7 0}$ & $\mathbf{1 0}$ & $\mathbf{6 5}$ & $\mathbf{1 6 5}$ \\
\hline $\mathbf{2 0}$ & 1 & 2 & 2 & 2 & 6 & 6 & 6 & 6 & 6 & $\mathbf{1 0}$ & 10 & 10 & 6 & 6 & 10 & 10 & 0 & 6 & 10 \\
\hline $\mathbf{3 0}$ & 0 & 2 & 3 & 4 & 5 & 0 & 5 & 5 & 5 & 0 & 0 & 5 & 5 & 5 & 5 & 5 & 0 & 5 & 5 \\
\hline $\mathbf{4 0}$ & 0 & 0 & 3 & 4 & 0 & 0 & 4 & 4 & 4 & 0 & 0 & 4 & 4 & 4 & 4 & 4 & 0 & 0 & 4 \\
\hline $\mathbf{5 0}$ & 0 & 0 & 0 & 4 & 0 & 0 & 7 & 7 & 7 & 0 & 0 & 7 & 7 & 7 & 7 & 7 & 0 & 0 & 7 \\
\hline $\mathbf{6 0}$ & 0 & 0 & 0 & 0 & 5 & 0 & 7 & 8 & 7 & 0 & 0 & 7 & 7 & 7 & 7 & 7 & 0 & 18 & 7 \\
\hline $\mathbf{7 0}$ & 0 & 2 & 2 & 2 & 5 & 6 & 5 & 8 & 9 & 0 & 0 & 9 & 5 & 9 & 9 & 5 & 0 & 5 & 9 \\
\hline $\mathbf{8 0}$ & 0 & 0 & 0 & 0 & 0 & 0 & 7 & 8 & 13 & 0 & 0 & 13 & 13 & 14 & 13 & 13 & 0 & 0 & 13 \\
\hline $\mathbf{9 0}$ & 0 & 0 & 0 & 0 & 0 & 0 & 7 & 8 & 14 & 0 & 0 & 14 & 14 & 14 & 14 & 14 & 0 & 0 & 14 \\
\hline $\mathbf{1 0 0}$ & 0 & 0 & 0 & 0 & 0 & 0 & 8 & 8 & 9 & 0 & 0 & 15 & 14 & 14 & 15 & 15 & 0 & 0 & 15 \\
\hline $\mathbf{1 1 0}$ & 0 & 0 & 0 & 0 & 0 & 0 & 9 & 9 & 9 & 10 & 11 & 15 & 9 & 9 & 15 & 15 & 0 & 0 & 15 \\
\hline $\mathbf{1 2 0}$ & 0 & 0 & 0 & 0 & 0 & 0 & 12 & 12 & 12 & 0 & 11 & 12 & 12 & 12 & 12 & 12 & 0 & 0 & 12 \\
\hline $\mathbf{1 3 0}$ & 0 & 0 & 0 & 0 & 0 & 0 & 15 & 15 & 15 & 0 & 0 & 12 & 15 & 15 & 15 & 16 & 0 & 0 & 15 \\
\hline $\mathbf{1 4 0}$ & 0 & 0 & 0 & 0 & 0 & 0 & 15 & 15 & 15 & 0 & 0 & 15 & 13 & 15 & 15 & 16 & 0 & 0 & 15 \\
\hline $\mathbf{1 5 0}$ & 0 & 0 & 0 & 0 & 0 & 0 & 8 & 8 & 15 & 0 & 0 & 15 & 13 & 14 & 15 & 13 & 0 & 0 & 15 \\
\hline $\mathbf{1 6 0}$ & 0 & 0 & 0 & 0 & 0 & 0 & 14 & 14 & 9 & 0 & 0 & 12 & 13 & 14 & 15 & 12 & 0 & 0 & 19 \\
\hline $\mathbf{1 7 0}$ & 0 & 0 & 0 & 0 & 0 & 0 & 12 & 12 & 12 & 0 & 0 & 12 & 12 & 12 & 12 & 16 & 0 & 0 & 12 \\
\hline $\mathbf{1 0}$ & 1 & 1 & 1 & 1 & 1 & 1 & 1 & 1 & 1 & 1 & 1 & 1 & 1 & 1 & 1 & 1 & 17 & 1 & 1 \\
\hline $\mathbf{6 5}$ & 0 & 0 & 0 & 0 & 5 & 0 & 5 & 5 & 5 & 0 & 0 & 5 & 5 & 5 & 5 & 5 & 0 & 18 & 5 \\
\hline $\mathbf{1 6 5}$ & 0 & 0 & 0 & 0 & 0 & 0 & 15 & 15 & 15 & 0 & 0 & 15 & 15 & 15 & 15 & 15 & 0 & 0 & 19 \\
\hline
\end{tabular}

Matriz Custo de Extensão de viagem Inicial (ft):

\begin{tabular}{|c|c|c|c|c|c|c|c|c|c|c|c|c|c|c|c|c|c|c|c|}
\hline Nós & 20 & 30 & 40 & 50 & 60 & 70 & 80 & 90 & 100 & 110 & 120 & 130 & 140 & 150 & 160 & 170 & 10 & 65 & 165 \\
\hline 20 & 0 & 12000 & 228200 & 228200 & 228200 & 12000 & 228200 & 228200 & 228200 & 12000 & 228200 & 228200 & 228200 & 228200 & 228200 & 228200 & 228200 & 228200 & 228200 \\
\hline 30 & 228200 & 0 & 6000 & 9000 & 6000 & 228200 & 228200 & 228200 & 228200 & 228200 & 228200 & 228200 & 228200 & 228200 & 228200 & 228200 & 228200 & 228200 & 228200 \\
\hline 40 & 228200 & 228200 & 0 & 6000 & 228200 & 228200 & 228200 & 228200 & 228200 & 228200 & 228200 & 228200 & 228200 & 228200 & 228200 & 228200 & 228200 & 228200 & 228200 \\
\hline 50 & 228200 & 228200 & 228200 & 0 & 228200 & 228200 & 6000 & 228200 & 228200 & 228200 & 228200 & 228200 & 12000 & 228200 & 228200 & 228200 & 228200 & 228200 & 228200 \\
\hline 60 & 228200 & 228200 & 228200 & 228200 & 0 & 228200 & 60000 & 6000 & 228200 & 228200 & 228200 & 228200 & 228200 & 228200 & 228200 & 228200 & 228200 & $\left.\begin{array}{lll}1 & 0 & 0\end{array}\right]$ & 228200 \\
\hline 70 & 228200 & 90000 & 228200 & 228200 & 6000 & 0 & 228200 & 6000 & 6000 & 228200 & 228200 & 228200 & 228200 & 228200 & 228200 & 228200 & 228200 & 228200 & 228200 \\
\hline 80 & 228200 & 228200 & 228200 & 228200 & 228200 & 228200 & 0 & 6000 & 228200 & 228200 & 228200 & 228200 & 6000 & 6000 & 228200 & 228200 & 228200 & 228200 & 228200 \\
\hline 90 & 228200 & 228200 & 228200 & 228200 & 228200 & 228200 & 6000 & 0 & 60000 & 228200 & 228200 & 228200 & 228200 & 6000 & 228200 & 228200 & 228200 & 228200 & 228200 \\
\hline 100 & 228200 & 228200 & 228200 & 228200 & 228200 & 228200 & 228200 & 6000 & 0 & 228200 & 228200 & 228200 & 228200 & 6000 & 6000 & 228200 & 228200 & 228200 & 228200 \\
\hline 110 & 228200 & 228200 & 228200 & 228200 & 228200 & 228200 & 228200 & 228200 & 6000 & 0 & 6000 & 228200 & 228200 & 228200 & 6000 & 228200 & 228200 & 228200 & 228200 \\
\hline 120 & 228200 & 228200 & 228200 & 228200 & 228200 & 228200 & 228200 & 228200 & 228200 & 228200 & 0 & 60000 & 228200 & 228200 & 228200 & 228200 & 228200 & 228200 & 228200 \\
\hline 130 & 228200 & 228200 & 228200 & 228200 & 228200 & 228200 & 228200 & 228200 & 228200 & 228200 & 228200 & 0 & 228200 & 228200 & 6000 & 6000 & 228200 & 228200 & 228200 \\
\hline 140 & 228200 & 228200 & 228200 & 228200 & 228200 & 228200 & 228200 & 228200 & 228200 & 228200 & 228200 & 228200 & 0 & 228200 & 6000 & 12000 & 228200 & 228200 & 228200 \\
\hline 150 & 228200 & 228200 & 228200 & 228200 & 228200 & 228200 & 6000 & 6000 & 228200 & 228200 & 228200 & 228200 & 6000 & 0 & 60000 & 228200 & 228200 & 228200 & 228200 \\
\hline 160 & 228200 & 228200 & 228200 & 228200 & 228200 & 228200 & 228200 & 228200 & 6000 & 228200 & 228200 & 6000 & 6000 & 6000 & 0 & 228200 & 228200 & 228200 & $\begin{array}{lll}1 & 0 & 0\end{array}$ \\
\hline 170 & 228200 & 228200 & 228200 & 228200 & 228200 & 228200 & 228200 & 228200 & 228200 & 228200 & 228200 & 6000 & 228200 & 228200 & 228200 & 0 & 228200 & 228200 & 228200 \\
\hline 10 & 0 & 228200 & 228200 & 228200 & 228200 & 228200 & 228200 & 228200 & 228200 & 228200 & 228200 & 228200 & 228200 & 228200 & 228200 & 228200 & 0 & 228200 & 228200 \\
\hline 65 & 228200 & 228200 & 228200 & 228200 & $\begin{array}{lll}1 & 0 & 0\end{array}$ & 228200 & 228200 & 228200 & 228200 & 228200 & 228200 & 228200 & 228200 & 228200 & 228200 & 228200 & 228200 & 0 & 228200 \\
\hline 165 & 228200 & 228200 & 228200 & 228200 & 228200 & 228200 & 228200 & 228200 & 228200 & 228200 & 228200 & 228200 & 228200 & 228200 & $\begin{array}{lll}1 & 0 & 0\end{array}$ & 228200 & 228200 & 228200 & 0 \\
\hline
\end{tabular}


Estudo da Localização Otimizada de Equipamentos em Rede de Distribuição de Água para Deteç̧ão de Contaminação

Matriz Roteamento Inicial 2:

\begin{tabular}{|c|c|c|c|c|c|c|c|c|c|c|c|c|c|c|c|c|c|c|c|}
\hline Nós & $\mathbf{2 0}$ & $\mathbf{3 0}$ & $\mathbf{4 0}$ & $\mathbf{5 0}$ & $\mathbf{6 0}$ & $\mathbf{7 0}$ & $\mathbf{8 0}$ & $\mathbf{9 0}$ & $\mathbf{1 0 0}$ & $\mathbf{1 1 0}$ & $\mathbf{1 2 0}$ & $\mathbf{1 3 0}$ & $\mathbf{1 4 0}$ & $\mathbf{1 5 0}$ & $\mathbf{1 6 0}$ & $\mathbf{1 7 0}$ & $\mathbf{1 0}$ & $\mathbf{6 5}$ & $\mathbf{1 6 5}$ \\
\hline $\mathbf{2 0}$ & 1 & 2 & 0 & 0 & 0 & 6 & 0 & 0 & 0 & $\mathbf{1 0}$ & 0 & 0 & 0 & 0 & 0 & 0 & 0 & 0 & 0 \\
\hline $\mathbf{3 0}$ & 0 & 2 & 3 & 4 & 5 & 0 & 0 & 0 & 0 & 0 & 0 & 0 & 0 & 0 & 0 & 0 & 0 & 0 & 0 \\
\hline $\mathbf{4 0}$ & 0 & 0 & 3 & 4 & 0 & 0 & 0 & 0 & 0 & 0 & 0 & 0 & 0 & 0 & 0 & 0 & 0 & 0 & 0 \\
\hline $\mathbf{5 0}$ & 0 & 0 & 0 & 4 & 0 & 0 & 7 & 0 & 0 & 0 & 0 & 0 & 13 & 0 & 0 & 0 & 0 & 0 & 0 \\
\hline $\mathbf{6 0}$ & 0 & 0 & 0 & 0 & 5 & 0 & 7 & 8 & 0 & 0 & 0 & 0 & 0 & 0 & 0 & 0 & 0 & 18 & 0 \\
\hline $\mathbf{7 0}$ & 0 & 2 & 0 & 0 & 5 & 6 & 0 & 8 & 9 & 0 & 0 & 0 & 0 & 0 & 0 & 0 & 0 & 0 & 0 \\
\hline $\mathbf{8 0}$ & 0 & 0 & 0 & 0 & 0 & 0 & 7 & 8 & 0 & 0 & 0 & 0 & 13 & 14 & 0 & 0 & 0 & 0 & 0 \\
\hline $\mathbf{9 0}$ & 0 & 0 & 0 & 0 & 0 & 0 & 7 & 8 & 9 & 0 & 0 & 0 & 0 & 14 & 0 & 0 & 0 & 0 & 0 \\
\hline $\mathbf{1 0 0}$ & 0 & 0 & 0 & 0 & 0 & 0 & 0 & 8 & 9 & 0 & 0 & 0 & 0 & 14 & 15 & 0 & 0 & 0 & 0 \\
\hline $\mathbf{1 1 0}$ & 0 & 0 & 0 & 0 & 0 & 0 & 0 & 0 & 9 & 10 & 11 & 0 & 0 & 0 & 15 & 0 & 0 & 0 & 0 \\
\hline $\mathbf{1 2 0}$ & 0 & 0 & 0 & 0 & 0 & 0 & 0 & 0 & 0 & 0 & 11 & 12 & 0 & 0 & 0 & 0 & 0 & 0 & 0 \\
\hline $\mathbf{1 3 0}$ & 0 & 0 & 0 & 0 & 0 & 0 & 0 & 0 & 0 & 0 & 0 & 12 & 0 & 0 & 15 & 16 & 0 & 0 & 0 \\
\hline $\mathbf{1 4 0}$ & 0 & 0 & 0 & 0 & 0 & 0 & 0 & 0 & 0 & 0 & 0 & 0 & 13 & 0 & 15 & 16 & 0 & 0 & 0 \\
\hline $\mathbf{1 5 0}$ & 0 & 0 & 0 & 0 & 0 & 0 & 7 & 8 & 0 & 0 & 0 & 0 & 13 & 14 & 15 & 0 & 0 & 0 & 0 \\
\hline $\mathbf{1 6 0}$ & 0 & 0 & 0 & 0 & 0 & 0 & 0 & 0 & 9 & 0 & 0 & 12 & 13 & 14 & 15 & 0 & 0 & 0 & 19 \\
\hline $\mathbf{1 7 0}$ & 0 & 0 & 0 & 0 & 0 & 0 & 0 & 0 & 0 & 0 & 0 & 12 & 0 & 0 & 0 & 16 & 0 & 0 & 0 \\
\hline $\mathbf{1 0}$ & 1 & 0 & 0 & 0 & 0 & 0 & 0 & 0 & 0 & 0 & 0 & 0 & 0 & 0 & 0 & 0 & 17 & 0 & 0 \\
\hline $\mathbf{6 5}$ & 0 & 0 & 0 & 0 & 5 & 0 & 0 & 0 & 0 & 0 & 0 & 0 & 0 & 0 & 0 & 0 & 0 & 18 & 0 \\
\hline $\mathbf{1 6 5}$ & 0 & 0 & 0 & 0 & 0 & 0 & 0 & 0 & 0 & 0 & 0 & 0 & 0 & 0 & 15 & 0 & 0 & 0 & 19 \\
\hline
\end{tabular}

Matriz Custo de Extensão de viagem Final (ft) (Caminhos Mais Curtos):

\begin{tabular}{|c|c|c|c|c|c|c|c|c|c|c|c|c|c|c|c|c|c|c|c|}
\hline Nós & 20 & 30 & 40 & 50 & 60 & 70 & 80 & 90 & 100 & 110 & 120 & 130 & 140 & 150 & 160 & 170 & 10 & 65 & 165 \\
\hline 20 & 0 & 2000 & 18000 & 21000 & 18000 & 12000 & 24000 & 18000 & 18000 & 12000 & 18000 & 24000 & 24000 & 24000 & 18000 & 30000 & 228200 & 18100 & 18100 \\
\hline 30 & 28200 & 0 & 6000 & 90000 & 6000 & 228200 & 12000 & 12000 & 18000 & 228200 & 228200 & 30000 & 18000 & 18000 & 24000 & 30000 & 228200 & 6100 & 24100 \\
\hline 40 & 228200 & 228200 & 0 & 6000 & 228200 & 228200 & 12000 & 18000 & 24000 & 228200 & 228200 & 30000 & 18000 & 18000 & 24000 & 30000 & 228200 & 228200 & 24100 \\
\hline 50 & 8200 & 228200 & 228200 & 0 & 228200 & 228200 & 6000 & 12000 & 18000 & 228200 & 228200 & 24000 & 12000 & 12000 & 18000 & 24000 & 228200 & 228200 & 18100 \\
\hline 60 & 228200 & 228200 & 228200 & 228200 & 0 & 228200 & 6000 & 6000 & 12000 & 228200 & 228200 & 24000 & 12000 & 12000 & 18000 & 24000 & 228200 & $\begin{array}{lll}1 & 0 & 0\end{array}$ & 18100 \\
\hline 70 & 228200 & 9000 & 15000 & 18000 & 6000 & 0 & 12000 & 6000 & 6000 & 228200 & 228200 & 18000 & 18000 & 12000 & 12000 & 24000 & 228200 & 6100 & 12100 \\
\hline 80 & 228200 & 228200 & 228200 & 228200 & 228200 & 228200 & 0 & 6000 & 12000 & 228200 & 228200 & 18000 & 6000 & 6000 & 12000 & 18000 & 228200 & 228200 & 12100 \\
\hline 90 & 228200 & 228200 & 228200 & 228200 & 228200 & 228200 & 6000 & 0 & 6000 & 228200 & 228200 & 18000 & 12000 & 6000 & 12000 & 24000 & 228200 & 228200 & 12100 \\
\hline 100 & 228200 & 228200 & 228200 & 228200 & 228200 & 228200 & 12000 & 60000 & 0 & 228200 & 228200 & 12000 & 12000 & 6000 & 6000 & 18000 & 228200 & 228200 & 6100 \\
\hline 110 & 228200 & 228200 & 228200 & 228200 & 228200 & 228200 & 18000 & 12000 & 6000 & 0 & 6000 & 12000 & 12000 & 12000 & 6000 & 18000 & 228200 & 228200 & 6100 \\
\hline 120 & 228200 & 228200 & 228200 & 228200 & 228200 & 228200 & 24000 & 24000 & 18000 & 228200 & 0 & 6000 & 18000 & 18000 & 12000 & 12000 & 228200 & 228200 & 12100 \\
\hline 130 & 228200 & 228200 & 228200 & 228200 & 228200 & 228200 & 18000 & 18000 & 12000 & 228200 & 228200 & 0 & 12000 & 12000 & 6000 & 6000 & 228200 & 228200 & 6100 \\
\hline 140 & 228200 & 228200 & 228200 & 228200 & 228200 & 228200 & 18000 & 18000 & 12000 & 228200 & 228200 & 12000 & 0 & 12000 & 6000 & 12000 & 228200 & 228200 & 6100 \\
\hline 150 & 228200 & 228200 & 228200 & 228200 & 228200 & 228200 & 6000 & 6000 & 12000 & 228200 & 228200 & 12000 & 6000 & 0 & 6000 & 18000 & 228200 & 228200 & 6100 \\
\hline 160 & 228200 & 228200 & 228200 & 228200 & 228200 & 228200 & 12000 & 12000 & 6000 & 228200 & 228200 & 6000 & 6000 & 6000 & 0 & 12000 & 228200 & 228200 & $\begin{array}{lll}1 & 0 & 0\end{array}$ \\
\hline 170 & 228200 & 228200 & 228200 & 228200 & 228200 & 228200 & 24000 & 24000 & 18000 & 228200 & 228200 & 6000 & 18000 & 18000 & 12000 & 0 & 228200 & 228200 & 12100 \\
\hline 10 & 0 & 12000 & 18000 & 21000 & 18000 & 12000 & 24000 & 18000 & 18000 & 12000 & 18000 & 24000 & 24000 & 24000 & 18000 & 30000 & 0 & 18100 & 18100 \\
\hline 65 & 228200 & 228200 & 228200 & 228200 & $\begin{array}{lll}1 & 0 & 0\end{array}$ & 228200 & 6100 & 6100 & 12100 & 228200 & 228200 & 24100 & 12100 & 12100 & 18100 & 24100 & 228200 & 0 & 18200 \\
\hline 165 & 228200 & 228200 & 228200 & 228200 & 228200 & 228200 & 12100 & 12100 & 6100 & 228200 & 228200 & 6100 & 6100 & 6100 & 100 & 12100 & 228200 & 228200 & 0 \\
\hline
\end{tabular}


Estudo da Localização Otimizada de Equipamentos em Rede de Distribuição de Água para Deteç̧ão de Contaminação

Matriz Roteamento Final 2:

\begin{tabular}{|c|c|c|c|c|c|c|c|c|c|c|c|c|c|c|c|c|c|c|c|}
\hline Nós & $\mathbf{2 0}$ & $\mathbf{3 0}$ & $\mathbf{4 0}$ & $\mathbf{5 0}$ & $\mathbf{6 0}$ & $\mathbf{7 0}$ & $\mathbf{8 0}$ & $\mathbf{9 0}$ & $\mathbf{1 0 0}$ & $\mathbf{1 1 0}$ & $\mathbf{1 2 0}$ & $\mathbf{1 3 0}$ & $\mathbf{1 4 0}$ & $\mathbf{1 5 0}$ & $\mathbf{1 6 0}$ & $\mathbf{1 7 0}$ & $\mathbf{1 0}$ & $\mathbf{6 5}$ & $\mathbf{1 6 5}$ \\
\hline $\mathbf{2 0}$ & 1 & 2 & 2 & 2 & 2 & 6 & 2 & 6 & 6 & $\mathbf{1 0}$ & 10 & 10 & 10 & 6 & 10 & 10 & 0 & 2 & 10 \\
\hline $\mathbf{3 0}$ & 0 & 2 & 3 & 4 & 5 & 0 & 5 & 5 & 5 & 0 & 0 & 5 & 5 & 5 & 5 & 5 & 0 & 5 & 5 \\
\hline $\mathbf{4 0}$ & 0 & 0 & 3 & 4 & 0 & 0 & 4 & 4 & 4 & 0 & 0 & 4 & 4 & 4 & 4 & 4 & 0 & 0 & 4 \\
\hline $\mathbf{5 0}$ & 0 & 0 & 0 & 4 & 0 & 0 & 7 & 7 & 7 & 0 & 0 & 13 & 13 & 7 & 13 & 13 & 0 & 0 & 13 \\
\hline $\mathbf{6 0}$ & 0 & 0 & 0 & 0 & 5 & 0 & 7 & 8 & 8 & 0 & 0 & 8 & 7 & 7 & 8 & 7 & 0 & 18 & 8 \\
\hline $\mathbf{7 0}$ & 0 & 2 & 2 & 2 & 5 & 6 & 5 & 8 & 9 & 0 & 0 & 9 & 5 & 8 & 9 & 9 & 0 & 5 & 9 \\
\hline $\mathbf{8 0}$ & 0 & 0 & 0 & 0 & 0 & 0 & 7 & 8 & 8 & 0 & 0 & 13 & 13 & 14 & 13 & 13 & 0 & 0 & 13 \\
\hline $\mathbf{9 0}$ & 0 & 0 & 0 & 0 & 0 & 0 & 7 & 8 & 9 & 0 & 0 & 9 & 7 & 14 & 9 & 7 & 0 & 0 & 9 \\
\hline $\mathbf{1 0 0}$ & 0 & 0 & 0 & 0 & 0 & 0 & 8 & 8 & 9 & 0 & 0 & 15 & 14 & 14 & 15 & 15 & 0 & 0 & 15 \\
\hline $\mathbf{1 1 0}$ & 0 & 0 & 0 & 0 & 0 & 0 & 9 & 9 & 9 & 10 & 11 & 11 & 15 & 9 & 15 & 11 & 0 & 0 & 15 \\
\hline $\mathbf{1 2 0}$ & 0 & 0 & 0 & 0 & 0 & 0 & 12 & 12 & 12 & 0 & 11 & 12 & 12 & 12 & 12 & 12 & 0 & 0 & 12 \\
\hline $\mathbf{1 3 0}$ & 0 & 0 & 0 & 0 & 0 & 0 & 15 & 15 & 15 & 0 & 0 & 12 & 15 & 15 & 15 & 16 & 0 & 0 & 15 \\
\hline $\mathbf{1 4 0}$ & 0 & 0 & 0 & 0 & 0 & 0 & 15 & 15 & 15 & 0 & 0 & 15 & 13 & 15 & 15 & 16 & 0 & 0 & 15 \\
\hline $\mathbf{1 5 0}$ & 0 & 0 & 0 & 0 & 0 & 0 & 7 & 8 & 8 & 0 & 0 & 15 & 13 & 14 & 15 & 13 & 0 & 0 & 15 \\
\hline $\mathbf{1 6 0}$ & 0 & 0 & 0 & 0 & 0 & 0 & 14 & 9 & 9 & 0 & 0 & 12 & 13 & 14 & 15 & 12 & 0 & 0 & 19 \\
\hline $\mathbf{1 7 0}$ & 0 & 0 & 0 & 0 & 0 & 0 & 12 & 12 & 12 & 0 & 0 & 12 & 12 & 12 & 12 & 16 & 0 & 0 & 12 \\
\hline $\mathbf{1 0}$ & 1 & 1 & 1 & 1 & 1 & 1 & 1 & 1 & 1 & 1 & 1 & 1 & 1 & 1 & 1 & 1 & 17 & 1 & 1 \\
\hline $\mathbf{6 5}$ & 0 & 0 & 0 & 0 & 5 & 0 & 5 & 5 & 5 & 0 & 0 & 5 & 5 & 5 & 5 & 5 & 0 & 18 & 5 \\
\hline $\mathbf{1 6 5}$ & 0 & 0 & 0 & 0 & 0 & 0 & 15 & 15 & 15 & 0 & 0 & 15 & 15 & 15 & 15 & 15 & 0 & 0 & 19 \\
\hline
\end{tabular}

Matriz Poluição para Volume Consumido (nível de serviço: 1245.15m3):

\begin{tabular}{|c|c|c|c|c|c|c|c|c|c|c|c|c|c|c|c|c|c|c|c|}
\hline Nós & $\mathbf{2 0}$ & $\mathbf{3 0}$ & $\mathbf{4 0}$ & $\mathbf{5 0}$ & $\mathbf{6 0}$ & $\mathbf{7 0}$ & $\mathbf{8 0}$ & $\mathbf{9 0}$ & $\mathbf{1 0 0}$ & $\mathbf{1 1 0}$ & $\mathbf{1 2 0}$ & $\mathbf{1 3 0}$ & $\mathbf{1 4 0}$ & $\mathbf{1 5 0}$ & $\mathbf{1 6 0}$ & $\mathbf{1 7 0}$ & $\mathbf{1 0}$ & $\mathbf{6 5}$ & $\mathbf{1 6 5}$ \\
\hline $\mathbf{2 0}$ & 1 & 1 & 1 & 0 & 1 & 1 & 0 & 1 & 1 & 1 & 1 & 0 & 0 & 0 & 1 & 0 & 0 & 1 & 1 \\
\hline $\mathbf{3 0}$ & 0 & 1 & 1 & 1 & 1 & 0 & 1 & 1 & 0 & 0 & 0 & 0 & 0 & 0 & 0 & 0 & 0 & 1 & 0 \\
\hline $\mathbf{4 0}$ & 0 & 0 & 1 & 1 & 0 & 0 & 1 & 0 & 0 & 0 & 0 & 0 & 1 & 0 & 1 & 0 & 0 & 0 & 1 \\
\hline $\mathbf{5 0}$ & 0 & 0 & 0 & 1 & 0 & 0 & 1 & 0 & 0 & 0 & 0 & 0 & 1 & 1 & 1 & 0 & 0 & 0 & 1 \\
\hline $\mathbf{6 0}$ & 0 & 0 & 0 & 0 & 1 & 0 & 1 & 1 & 0 & 0 & 0 & 0 & 1 & 0 & 0 & 0 & 0 & 1 & 0 \\
\hline $\mathbf{7 0}$ & 0 & 0 & 0 & 0 & 1 & 1 & 1 & 1 & 1 & 0 & 0 & 0 & 0 & 0 & 1 & 0 & 0 & 1 & 1 \\
\hline $\mathbf{8 0}$ & 0 & 0 & 0 & 0 & 0 & 0 & 1 & 0 & 0 & 0 & 0 & 0 & 1 & 1 & 1 & 1 & 0 & 0 & 1 \\
\hline $\mathbf{9 0}$ & 0 & 0 & 0 & 0 & 0 & 0 & 0 & 1 & 0 & 0 & 0 & 0 & 0 & 0 & 0 & 0 & 0 & 0 & 0 \\
\hline $\mathbf{1 0 0}$ & 0 & 0 & 0 & 0 & 0 & 0 & 0 & 1 & 1 & 0 & 0 & 1 & 0 & 1 & 1 & 0 & 0 & 0 & 1 \\
\hline $\mathbf{1 1 0}$ & 0 & 0 & 0 & 0 & 0 & 0 & 0 & 0 & 1 & 1 & 1 & 1 & 0 & 1 & 1 & 0 & 0 & 0 & 1 \\
\hline $\mathbf{1 2 0}$ & 0 & 0 & 0 & 0 & 0 & 0 & 0 & 0 & 0 & 0 & 1 & 1 & 0 & 0 & 0 & 1 & 0 & 0 & 0 \\
\hline $\mathbf{1 3 0}$ & 0 & 0 & 0 & 0 & 0 & 0 & 0 & 0 & 0 & 0 & 0 & 1 & 0 & 0 & 0 & 1 & 0 & 0 & 0 \\
\hline $\mathbf{1 4 0}$ & 0 & 0 & 0 & 0 & 0 & 0 & 0 & 0 & 0 & 0 & 0 & 1 & 1 & 0 & 1 & 1 & 0 & 0 & 1 \\
\hline $\mathbf{1 5 0}$ & 0 & 0 & 0 & 0 & 0 & 0 & 0 & 0 & 0 & 0 & 0 & 1 & 1 & 1 & 1 & 0 & 0 & 0 & 1 \\
\hline $\mathbf{1 6 0}$ & 0 & 0 & 0 & 0 & 0 & 0 & 0 & 0 & 0 & 0 & 0 & 1 & 0 & 0 & 1 & 0 & 0 & 0 & 1 \\
\hline $\mathbf{1 7 0}$ & 0 & 0 & 0 & 0 & 0 & 0 & 0 & 0 & 0 & 0 & 0 & 0 & 0 & 0 & 0 & 1 & 0 & 0 & 0 \\
\hline $\mathbf{1 0}$ & 1 & 1 & 1 & 0 & 1 & 1 & 0 & 1 & 1 & 1 & 1 & 0 & 0 & 0 & 1 & 0 & 0 & 1 & 1 \\
\hline $\mathbf{6 5}$ & 0 & 0 & 0 & 0 & 1 & 0 & 1 & 1 & 0 & 0 & 0 & 0 & 1 & 0 & 0 & 0 & 0 & 0 & 0 \\
\hline $\mathbf{1 6 5}$ & 0 & 0 & 0 & 0 & 0 & 0 & 0 & 0 & 0 & 0 & 0 & 1 & 0 & 0 & 1 & 0 & 0 & 0 & 0 \\
\hline
\end{tabular}


Estudo da Localização Otimizada de Equipamentos em Rede

de Distribuição de Água para Deteç̧ão de Contaminação

Matriz Poluição para Tempo de Detecção (nível de serviço: 6.00h.)

\begin{tabular}{|c|c|c|c|c|c|c|c|c|c|c|c|c|c|c|c|c|c|c|c|}
\hline Nós & $\mathbf{2 0}$ & $\mathbf{3 0}$ & $\mathbf{4 0}$ & $\mathbf{5 0}$ & $\mathbf{6 0}$ & $\mathbf{7 0}$ & $\mathbf{8 0}$ & $\mathbf{9 0}$ & $\mathbf{1 0 0}$ & $\mathbf{1 1 0}$ & $\mathbf{1 2 0}$ & $\mathbf{1 3 0}$ & $\mathbf{1 4 0}$ & $\mathbf{1 5 0}$ & $\mathbf{1 6 0}$ & $\mathbf{1 7 0}$ & $\mathbf{1 0}$ & $\mathbf{6 5}$ & $\mathbf{1 6 5}$ \\
\hline $\mathbf{2 0}$ & 1 & 1 & 1 & $\mathbf{1}$ & 1 & 1 & 1 & 1 & 1 & 1 & 1 & 1 & 1 & 1 & 1 & 0 & 0 & 1 & 1 \\
\hline $\mathbf{3 0}$ & 0 & 1 & 1 & 1 & 1 & 0 & 1 & 1 & 0 & 0 & 0 & 0 & 1 & 0 & 0 & 0 & 0 & 1 & 0 \\
\hline $\mathbf{4 0}$ & 0 & 0 & 1 & 1 & 0 & 0 & 1 & 0 & 0 & 0 & 0 & 0 & 1 & 0 & 0 & 0 & 0 & 0 & 0 \\
\hline $\mathbf{5 0}$ & 0 & 0 & 0 & 1 & 0 & 0 & 1 & 0 & 0 & 0 & 0 & 0 & 1 & 0 & 1 & 0 & 0 & 0 & 1 \\
\hline $\mathbf{6 0}$ & 0 & 0 & 0 & 0 & 1 & 0 & 1 & 1 & 0 & 0 & 0 & 0 & 1 & 0 & 1 & 0 & 0 & 1 & 1 \\
\hline $\mathbf{7 0}$ & 0 & 0 & 0 & 0 & 1 & 1 & 1 & 1 & 1 & 0 & 0 & 1 & 1 & 1 & 1 & 0 & 0 & 1 & 1 \\
\hline $\mathbf{8 0}$ & 0 & 0 & 0 & 0 & 0 & 0 & 1 & 0 & 0 & 0 & 0 & 0 & 1 & 1 & 1 & 1 & 0 & 0 & 1 \\
\hline $\mathbf{9 0}$ & 0 & 0 & 0 & 0 & 0 & 0 & 0 & 1 & 0 & 0 & 0 & 0 & 0 & 1 & 0 & 0 & 0 & 0 & 0 \\
\hline $\mathbf{1 0 0}$ & 0 & 0 & 0 & 0 & 0 & 0 & 0 & 1 & 1 & 0 & 0 & 1 & 1 & 1 & 1 & 0 & 0 & 0 & 1 \\
\hline $\mathbf{1 1 0}$ & 0 & 0 & 0 & 0 & 0 & 0 & 0 & 1 & 1 & 1 & 1 & 1 & 0 & 1 & 1 & 0 & 0 & 0 & 1 \\
\hline $\mathbf{1 2 0}$ & 0 & 0 & 0 & 0 & 0 & 0 & 0 & 0 & 0 & 0 & 1 & 1 & 0 & 0 & 0 & 0 & 0 & 0 & 0 \\
\hline $\mathbf{1 3 0}$ & 0 & 0 & 0 & 0 & 0 & 0 & 0 & 0 & 0 & 0 & 0 & 1 & 0 & 0 & 0 & 1 & 0 & 0 & 0 \\
\hline $\mathbf{1 4 0}$ & 0 & 0 & 0 & 0 & 0 & 0 & 0 & 0 & 0 & 0 & 0 & 1 & 1 & 0 & 1 & 1 & 0 & 0 & 1 \\
\hline $\mathbf{1 5 0}$ & 0 & 0 & 0 & 0 & 0 & 0 & 0 & 0 & 0 & 0 & 0 & 1 & 1 & 1 & 1 & 0 & 0 & 0 & 1 \\
\hline $\mathbf{1 6 0}$ & 0 & 0 & 0 & 0 & 0 & 0 & 0 & 0 & 0 & 0 & 0 & 1 & 0 & 0 & 1 & 0 & 0 & 0 & 1 \\
\hline $\mathbf{1 7 0}$ & 0 & 0 & 0 & 0 & 0 & 0 & 0 & 0 & 0 & 0 & 0 & 0 & 0 & 0 & 0 & 1 & 0 & 0 & 0 \\
\hline $\mathbf{1 0}$ & 1 & 1 & 1 & 1 & 1 & 1 & 1 & 1 & 1 & 1 & 1 & 1 & 1 & 1 & 1 & 0 & 1 & 1 & 1 \\
\hline $\mathbf{6 5}$ & 0 & 0 & 0 & 0 & 1 & 0 & 1 & 1 & 0 & 0 & 0 & 0 & 1 & 0 & 1 & 0 & 0 & 1 & 1 \\
\hline $\mathbf{1 6 5}$ & 0 & 0 & 0 & 0 & 0 & 0 & 0 & 0 & 0 & 0 & 0 & 1 & 0 & 0 & 1 & 0 & 0 & 0 & 1 \\
\hline
\end{tabular}

Matriz Poluição para Extensão da Contaminação (nível de serviço: 15.00\%):

\begin{tabular}{|c|c|c|c|c|c|c|c|c|c|c|c|c|c|c|c|c|c|c|c|c|c|c|c|}
\hline Nós & $\mathbf{2 0}$ & $\mathbf{3 0}$ & $\mathbf{4 0}$ & $\mathbf{5 0}$ & $\mathbf{6 0}$ & $\mathbf{7 0}$ & $\mathbf{8 0}$ & $\mathbf{9 0}$ & $\mathbf{1 0 0}$ & $\mathbf{1 1 0}$ & $\mathbf{1 2 0}$ & $\mathbf{1 3 0}$ & $\mathbf{1 4 0}$ & $\mathbf{1 5 0}$ & $\mathbf{1 6 0}$ & $\mathbf{1 7 0}$ & $\mathbf{1 0}$ & $\mathbf{6 5}$ & $\mathbf{1 6 5}$ \\
\hline $\mathbf{2 0}$ & 1 & 1 & 1 & 1 & 1 & 1 & 1 & 1 & 1 & 1 & 1 & 1 & 1 & 1 & 1 & 1 & 0 & 1 & 1 \\
\hline $\mathbf{3 0}$ & 0 & 1 & 1 & 1 & 1 & 0 & 1 & 1 & 1 & 0 & 0 & 1 & 1 & 1 & 1 & 1 & 0 & 1 & 1 \\
\hline $\mathbf{4 0}$ & 0 & 0 & 1 & 1 & 0 & 0 & 1 & 1 & 1 & 0 & 0 & 1 & 1 & 1 & 1 & 1 & 0 & 0 & 1 \\
\hline $\mathbf{5 0}$ & 0 & 0 & 0 & 1 & 0 & 0 & 1 & 1 & 1 & 0 & 0 & 1 & 1 & 1 & 1 & 1 & 0 & 0 & 1 \\
\hline $\mathbf{6 0}$ & 0 & 0 & 0 & 0 & 1 & 0 & 1 & 1 & 1 & 0 & 0 & 1 & 1 & 1 & 1 & 1 & 0 & 1 & 1 \\
\hline $\mathbf{7 0}$ & 0 & 1 & 1 & 1 & 1 & 1 & 1 & 1 & 1 & 0 & 0 & 1 & 1 & 1 & 1 & 1 & 0 & 1 & 1 \\
\hline $\mathbf{8 0}$ & 0 & 0 & 0 & 0 & 0 & 0 & 1 & 1 & 1 & 0 & 0 & 1 & 1 & 1 & 1 & 1 & 0 & 0 & 1 \\
\hline $\mathbf{9 0}$ & 0 & 0 & 0 & 0 & 0 & 0 & 1 & 1 & 1 & 0 & 0 & 1 & 1 & 1 & 1 & 1 & 0 & 0 & 1 \\
\hline $\mathbf{1 0 0}$ & 0 & 0 & 0 & 0 & 0 & 0 & 1 & 1 & 1 & 0 & 0 & 1 & 1 & 1 & 1 & 1 & 0 & 0 & 1 \\
\hline $\mathbf{1 1 0}$ & 0 & 0 & 0 & 0 & 0 & 0 & 1 & 1 & 1 & 1 & 1 & 1 & 1 & 1 & 1 & 1 & 0 & 0 & 1 \\
\hline $\mathbf{1 2 0}$ & 0 & 0 & 0 & 0 & 0 & 0 & 1 & 1 & 1 & 0 & 1 & 1 & 1 & 1 & 1 & 1 & 0 & 0 & 1 \\
\hline $\mathbf{1 3 0}$ & 0 & 0 & 0 & 0 & 0 & 0 & 1 & 1 & 1 & 0 & 0 & 1 & 1 & 1 & 1 & 1 & 0 & 0 & 1 \\
\hline $\mathbf{1 4 0}$ & 0 & 0 & 0 & 0 & 0 & 0 & 1 & 1 & 1 & 0 & 0 & 1 & 1 & 1 & 1 & 1 & 0 & 0 & 1 \\
\hline $\mathbf{1 5 0}$ & 0 & 0 & 0 & 0 & 0 & 0 & 1 & 1 & 1 & 0 & 0 & 1 & 1 & 1 & 1 & 1 & 0 & 0 & 1 \\
\hline $\mathbf{1 6 0}$ & 0 & 0 & 0 & 0 & 0 & 0 & 1 & 1 & 1 & 0 & 0 & 1 & 1 & 1 & 1 & 1 & 0 & 0 & 1 \\
\hline $\mathbf{1 7 0}$ & 0 & 0 & 0 & 0 & 0 & 0 & 1 & 1 & 1 & 0 & 0 & 1 & 1 & 1 & 1 & 1 & 0 & 0 & 1 \\
\hline $\mathbf{1 0}$ & 1 & 1 & 1 & 1 & 1 & 1 & 1 & 1 & 1 & 1 & 1 & 1 & 1 & 1 & 1 & 1 & 1 & 1 & 1 \\
\hline $\mathbf{6 5}$ & 0 & 0 & 0 & 0 & 1 & 0 & 1 & 1 & 1 & 0 & 0 & 1 & 1 & 1 & 1 & 1 & 0 & 1 & 1 \\
\hline $\mathbf{1 6 5}$ & 0 & 0 & 0 & 0 & 0 & 0 & 1 & 1 & 1 & 0 & 0 & 1 & 1 & 1 & 1 & 1 & 0 & 0 & 1 \\
\hline
\end{tabular}

Programa finalizado com sucesso!

Tempo decorrido: $13.12 \mathrm{~s}$. 


\section{Anexo VIII - PLEDECORDA_MOM.TXT (Rede Anytown): Saídas dos Nós escolhidos: \\ Nível de Serviço 1 - Volume Consumido: 1245,15m³}

\section{FRENTES PARETO GLOBAL}

Número de Equipamentos: 1 un.

Considerado acréscimo de 0.01 por detecções repetidas.
f1: f2: f3:
Nós de monitoramento(X[i]):
1: $\quad 12 \quad 13 \quad 19$
$160(14)$

Número de Equipamentos: 2 un.

Considerado acréscimo de 0.01 por detecções repetidas.

\begin{tabular}{llllll} 
& f1: & f2: & f3: & \multicolumn{2}{c}{ Nós de monitoramento (X[i]): } \\
1: & 16.04 & 15.07 & 19.19 & $160(14)$ & $90(7)$ \\
2: & 13.02 & 17.06 & 19.19 & $140(12)$ & $130(11)$ \\
3: & 15 & 17.03 & 19.19 & $130(11)$ & $80(6)$
\end{tabular}

Número de Equipamentos: 3 un.

Considerado acréscimo de 0.01 por detecções repetidas.

\begin{tabular}{lllllll} 
& f1: & f2: & f3: & \multicolumn{3}{c}{ Nós de monitoramento (X[i]): } \\
1: & 18.05 & 18.14 & 19.38 & $130(11)$ & $140(12)$ & $90(7)$ \\
2: & 18.1 & 17.16 & 19.38 & $130(11)$ & $160(14)$ & $90(7)$ \\
3: & 19.06 & 17.09 & 19.38 & $170(15)$ & $160(14)$ & $90(7)$
\end{tabular}

Número de Equipamentos: 4 un.

Considerado acréscimo de 0.01 por detecções repetidas.
f1: f2: f3:
Nós de monitoramento (X[i]):

$\begin{array}{llll}\text { 1: } & 19.13 & 18.2 & 19.57 \\ \text { 2: } & 19.09 & 19.17 & 19.57\end{array}$
$160(14)$
$140(12)$
$170(15)$
$90(7)$
2: $\quad 19.09 \quad 19.17 \quad 19.57$
$140(12)$
$130(11)$
$170(15)$
$90(7)$ 


$\begin{array}{llllllll}\text { 3: } & 19.14 & 18.19 & 19.57 & 160(14) & 130(11) & 170(15) & 90(7) \\ \text { 4: } & 19.17 & 17.22 & 19.57 & 160(14) & 165(18) & 170(15) & 90(7)\end{array}$

\section{FRENTES PARETO GLOBAL}

Número de Equipamentos: 1 un.

Não considerado acréscimo por detecções repetidas.
f1: f2: f3:
Nós de monitoramento (X[i]):
1: $\quad 12 \quad 13 \quad 19$
$160(14)$

Número de Equipamentos: 2 un.

Não considerado acréscimo por detecções repetidas.
f1: f2: f3:
Nós de monitoramento (X[i]):
1: $\quad 15 \quad 17 \quad 19$
$80(6)$
$130(11)$
2: $\quad 16 \quad 15 \quad 19$
$90(7)$
$160(14)$

Número de Equipamentos: 3 un.

Não considerado acréscimo por detecções repetidas.
f1: f2: f3:
Nós de monitoramento (X[i]):
1: $\quad 19 \quad 17 \quad 19$
$90(7)$
$160(14)$
$170(15)$
2: $\quad 18 \quad 18 \quad 19$
$80(6)$
$130(11)$
$90(7)$

Número de Equipamentos: 4 un.

Não considerado acréscimo por detecções repetidas.
f1: f2: f3:
Nós de monitoramento (X[i]):
1: $\quad 19 \quad 19 \quad 19$
$170(15)$
$130(11)$
$80(6)$
90 (7) 